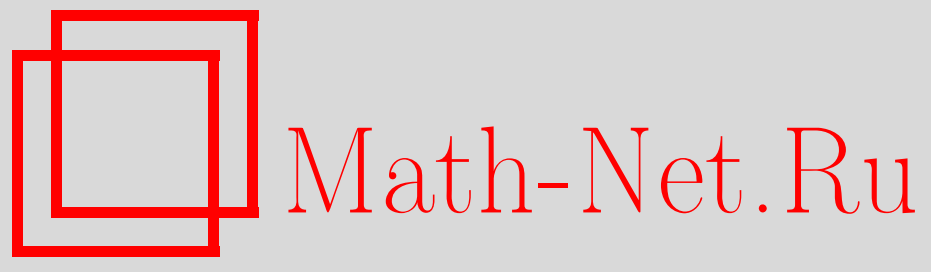

Г. Н. Пифтанкин, Д. В. Трещёв, Сепаратрисное отображение в гамильтоновых системах, УМH, 2007, том 62, выпуск 2, 3-108

DOI: https://doi.org/10.4213/rm6804

Использование Общероссийского математического портала Math-Net.Ru подразумевает, что вы прочитали и согласны с пользовательским соглашением http://www . mathnet.ru/rus/agreement

Параметры загрузки:

IP : 3.95 .254 .165

26 апреля 2023 г., 13:28:41

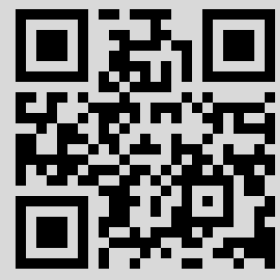


УДК 531.01

\section{Сепаратрисное отображение в гамильтоновых системах}

\section{Г. Н. Пифтанкин, Д. В. Трещёв}

Сепаратрисное отображение построено в нескольких классах задач гамильтоновой динамики. Полученные формулы применены для исследования двумерных симплектических отображений, близких к интегрируемым: построены эллиптические периодические траектории, проходящие через сепаратрисные лунки, даны оценки ширины стохастического слоя. В гамильтоновых системах с двумя с половиной степенями свободы доказана типичность диффузии Арнольда в априори неустойчивом случае и построены траектории с линейным по времени средним ростом энергии в задаче Мезера.

Библиография: 66 названий.

\section{СОДЕРЖАНИЕ}

Глава 1. Введение.......................................... 5

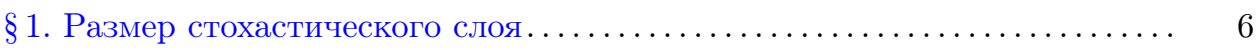

$\S 2$. Островки устойчивости в сепаратрисных лунках ............... 8

$\S 3$. Диффузия Арнольда: постановка проблемы.................... 11

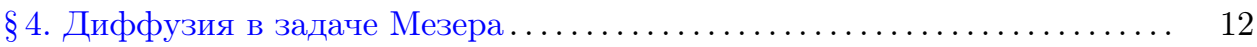

$\S 5$. Диффузия в априори неустойчивых системах .................. 14

Глава 2. Предварительные замечания .......................... 17

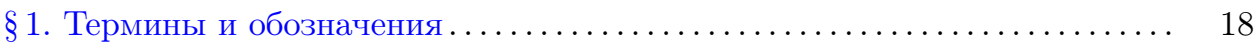

$\S 2$. Неподвижные точки двумерных симплектоморфизмов ........... 18

$\S 3$. Нормальные координаты в окрестности гиперболической неподвижной

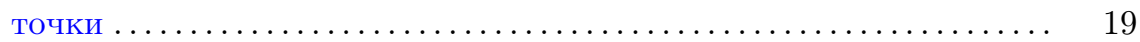

Глава 3. Сепаратрисное отображение Заславского.................. 20

$\S 1$. Определение и формулы . . . . . . . . . . . . . . . . . . . . . . . 20

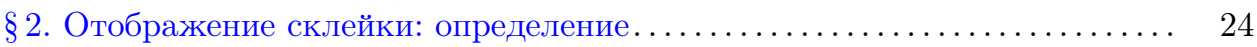

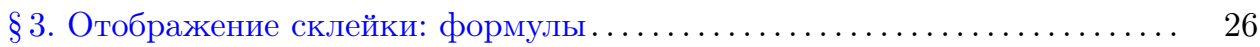

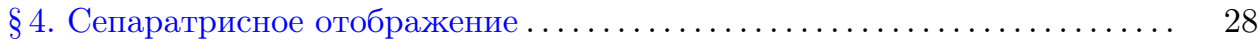

$\S 5$. Интеграл Пуанкаре-Мельникова ......................... 29

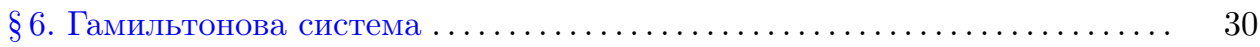

$\S 7$. Сепаратрисное отображение для маятника .................. 31

$\S 8$. Сепаратрисное отображение в общем случае ................. 32

(C) Г. Н. ПифтАНкин, Д. В. ТРЕщёв, 2007 
Глава 4. Сепаратрисное отображение Шильникова.................. 34

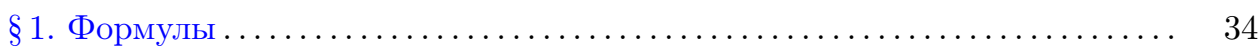

$\S 2$. Доказательство ................................... 36

Глава 5. Сепаратрисное отображение в задаче Мезера ............... 38

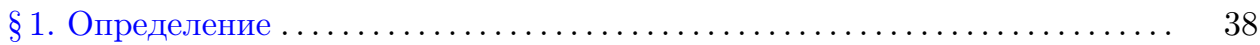

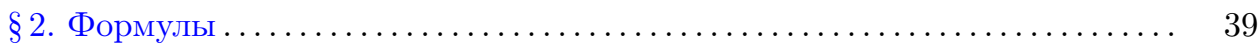

Глава 6. Сепаратрисное отображение в априори неустойчивых системах . 41

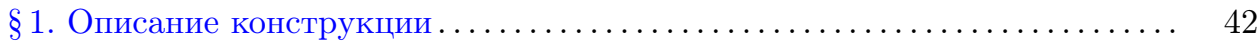

$\S 2$. Формулы для сепаратрисного отображения ................... 43

Глава 7. Размер стохастического слоя ...................... 46

$\S 1$. Теоремы о величине стохастического слоя.................. 46

$\S 2$. Стохастический слой в маятнике с колеблющейся точкой подвеса .... 51

$\S 3$. Теория КАМ и теорема Биркгофа...................... 52

Глава 8. Островки устойчивости в сепаратрисных лунках........... 56

$\S 1$. Следствия из обратимости ......................... 56

$\S 2$. Симметричные траектории периода $3 \ldots \ldots \ldots \ldots \ldots \ldots \ldots \ldots \ldots \ldots \ldots$

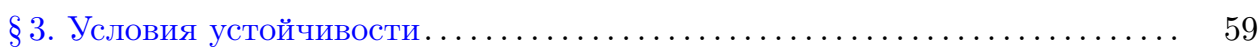

$\S 4$. ЭПЛ-траектории существуют......................... 59

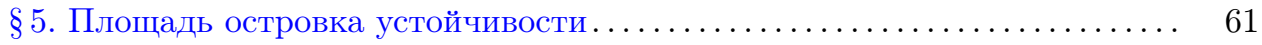

Глава 9. Рост энергии в задаче Мезера ......................... 61

$\S 1$. Формулировка результата......................... 61

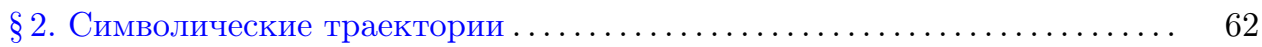

$\S 3$. Быстрые траектории Мезера......................... 66

Глава 10. Траектории в окрестности асимптотических многообразий в априори неустойчивых системах. ...................... 69

$\S 1$. Уравнения Рауса................................. 69

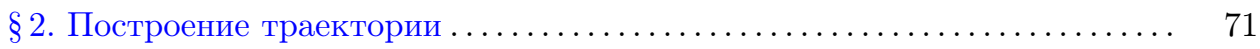

$\S 3$. Доказательство леммы о присоединении .................... 74

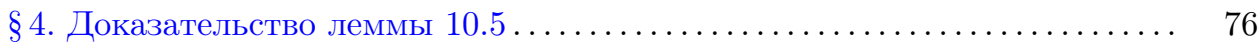

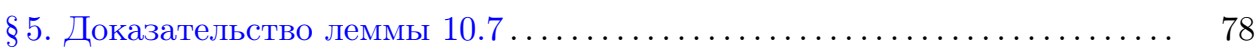

$\S 6$. Соотношения между постоянными ................... 79

Глава 11. Диффузия Арнольда в априори неустойчивых системах....... 79

$\S 1$. Предварительные соображения ...................... 79

$\S 2$. Четкие и нечеткие множества . . . . . . . . . . . . . . . . . . 80

$\S 3$. Прохождение через нерезонансный интервал ................. 84

$\S 4$. Прохождение через четкий существенный резонанс $\ldots \ldots \ldots \ldots \ldots \ldots \ldots \quad 86$

$\S 5$. Прохождение через нечеткий интервал ................... 92

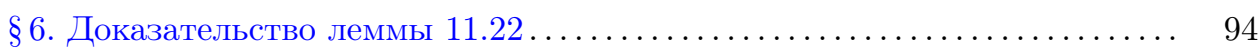

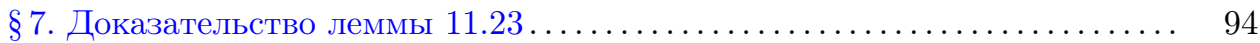

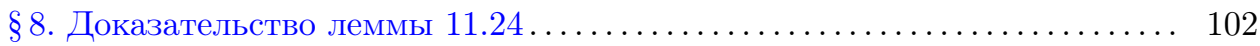




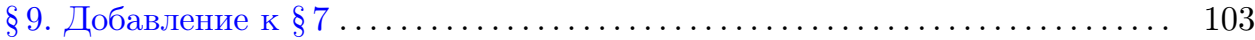

$\S 10$. Вспомогательные утверждения ......................... 104

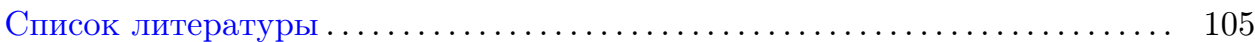

\section{Глава 1. Введение}

Сепаратрисное отображение было придумано как удобное средство для изучения динамики диффеоморфизма или потока в малой окрестности асимптотических многообразий гиперболических неподвижных точек или периодических решений. Далее решение, порождающее асимптотические многообразия, будет называться базовым. Траектории, не выходящие из таких окрестностей, стартуют в некоторой "фундаментальной" области, приближаются к базовому решению и затем возвращаются в фундаментальную область. При этом бо́льшую часть времени траектория проводит около базового решения. Естественная идея состоит в том, чтобы пропустить динамически неинтересную часть движения, расположенную около базового решения, и рассмотреть лишь индуцированное отображение на себя фундаментальной области в случае дискретного времени или некоторого сечения Пуанкаре для непрерывного времени. Это отображение и называется сепаратрисным.

Удобство сепаратрисного отображения как средства исследования динамики в окрестности асимптотических многообразий объясняется следующими обстоятельствами:

- существуют координаты, в которых сепаратрисное отображение принимает универсальный и относительно простой вид: “главная часть" плюс "малые ошибки",

- главная часть сепаратрисного отображения вычисляется явно в терминах невозмущенной системы и возмущения,

- "ошибки" явно оцениваются.

После того, как удобные формулы для сепаратрисного отображения получены, остается воспользоваться ими для исследования динамики. Таким образом результаты, представленные в настоящей статье, можно разделить на два класса: получение формул для различных сепаратрисных отображений и их применение. Как правило, мы будем рассматривать симплектические отображения и гамильтоновы потоки, хотя в ряде ситуаций аналогичные методы работают и для общих систем.

Существует два идеологически и технически различных класса систем, в которых сепаратрисное отображение позволило получить интересные динамические эффекты.

Первый класс - это системы близкие к интегрируемым. В невозмущенной интегрируемой системе асимптотические многообразия оказываются сдвоенными, а формулы для сепаратрисного отображения очень простыми. При малом возмущении в главном приближении по возмущающему параметру (традиционно, $\varepsilon)$ в $O(\varepsilon)$-окрестности асимптотических многообразий удается получить явные универсальные формулы для сепаратрисного отображения. Читатель, знакомый с проблематикой расщепления сепаратрис, конечно, догадывается, 
что ключевую роль в этих формулах играют периодические функции, определенные интегралом Пуанкаре-Мельникова. Сепаратрисные отображения такого типа впервые возникли в [1]. Далее мы их условно называем сепаратрисными отображениями Заславского.

Для систем второго класса не требуется близости к интегрируемости. Здесь объектом исследования служит динамика, сосредоточенная в малой окрестности одной гомоклинической (т.е. двоякоасимптотической к базовой) траектории. Окрестности указанного типа, конечно, как правило, не инвариантны, но целиком содержат достаточно большой набор траекторий. Сепаратрисное отображение позволяет получить довольно подробное описание этой динамики. Подобный подход был предложен в [2] и далее был успешно применен для построения символической динамики, исследования гомоклинических бифуркаций, исследования хаотических аттракторов и т.п. В этой ситуации мы будем говорить о сепаратрисном отображении Шильникова.

Первая половина статьи (главы 3-6) имеет технический характер. В них даны определения и получены формулы для сепаратрисных отображений в различных ситуациях. Во второй половине статьи даны приложения сепаратрисного отображения в задачах динамики. Остановимся на этих приложениях подробнее.

\section{§ 1. Размер стохастического слоя}

Пусть $T$ - гладкое симплектическое отображение двумерной области $D$ на себя. Предположим, что отображение $T$ близко к интегрируемому. (Точный смысл этому утверждению будет придан в 11 главы 3.) Предположим также, что $T$ имеет гиперболическую неподвижную точку $\sigma$, сепаратрисы которой ведут себя так, как показано на рис. 1.1. Три инвариантные кривые $\gamma_{ \pm}$и $\gamma_{0}$, ближайшие к сепаратрисам, образуют границу стохастического слоя. Ширина $w$ стохастического слоя - одна из важных величин, характеризующих хаотические свойства $T$ в окрестности сепаратрис. Определение величины $w$, а также ширины $d$ сепаратрисной лунки $D$ будет дано в $\S 1$ главы 7 .

В главе 7 мы покажем, что при некоторых естественных предположениях выполняется следующая формула:

$$
w / d \sim 1 / \ln \mu \text {. }
$$

Здесь $\mu>1$ - больший мультипликатор гиперболической неподвижной точки $\sigma$. Символ $\sim$ означает, что для гладкого семейства $T_{\varepsilon}, 0 \leqslant \varepsilon \leqslant \varepsilon_{0}$, гладких симплектических отображений, где $T_{0}$ интегрируемо,

$$
C_{1} / \ln \mu(\varepsilon)<w(\varepsilon) / d(\varepsilon)<C_{2} / \ln \mu(\varepsilon) .
$$

Если $\ln \mu \sim 1$, мы видим, что величины $w$ и $d$ имеют одинаковый порядок. В то же время, бывают ситуации, когда при малых $\varepsilon$ мультипликатор $\mu$ близок к единице. Рассмотрим, например, разрушение резонансной инвариантной окружности интегрируемого отображения

$$
T_{0}(x, y)=(x+\nu(y), y)
$$




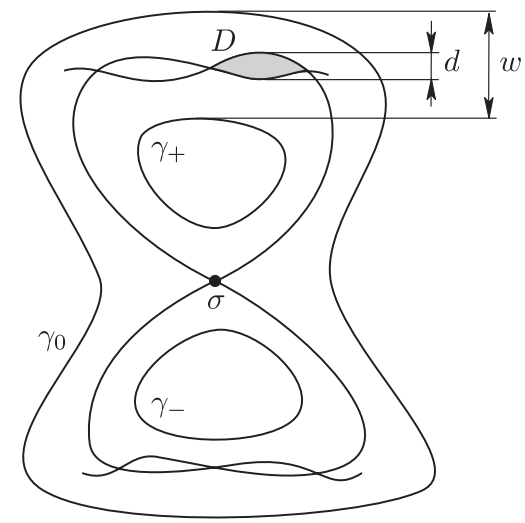

Рис. 1.1. Окрестность сепаратрис гиперболической точки $\sigma$. Размер стохастического слоя.

цилиндра $Z=\{x \bmod 2 \pi, y\}$ на себя при точном симплектическом возмущении. Пусть для простоты резонанс - простейший, т.е. исходная окружность задается условием $y=y_{0}, \nu\left(y_{0}\right)=0$. Возмущенное отображение выглядит следующим образом:

$$
T_{\varepsilon}(x, y)=(x+\nu(y)+\varepsilon f(x, y), y+\varepsilon g(x, y))+O\left(\varepsilon^{2}\right) .
$$

В перенормированных переменных $(x \bmod 2 \pi, \eta), y=y_{0}+\sqrt{\varepsilon} \eta$, отображение $T_{\varepsilon}$ имеет вид

$$
(x, \eta) \mapsto\left(x+\sqrt{\varepsilon} \nu_{0}^{\prime}, \eta-\sqrt{\varepsilon} v(x)\right)+O(\varepsilon),
$$

где $\nu_{0}^{\prime}=d \nu /\left.d y\right|_{y=0}$ и $v=-g\left(x, y_{0}\right)$. Из условия точной симплектичности вытекает равенство нулю среднего функции $v$ по угловой переменной $x$, следовательно, для некоторой периодической функции $V(x)$ выполняется равенство $v(x)=\partial V(x) / \partial x$. Отображение (1.2) отличается лишь в порядке $O(\varepsilon)$ от сдвига на время $\sqrt{\varepsilon}$ вдоль решений гамильтоновой системы с гамильтонианом $\nu_{0}^{\prime} \eta^{2} / 2+V(x)$.

Пусть $x_{0}$ - невырожденный локальный максимум потенциала $V$. Тогда несложно показать, что отображение (1.2) имеет гиперболическую неподвижную точку $(x, \eta)=\left(x_{0}, 0\right)+O(\sqrt{\varepsilon})$. Мультипликатор $\mu>1$ в этой точке следующий:

$$
\mu=\exp \left(\sqrt{-\nu_{0}^{\prime} V_{0}^{\prime \prime} \varepsilon}\right), \quad V_{0}^{\prime \prime}=\partial^{2} V /\left.\partial x^{2}\right|_{x=x_{0}}<0 .
$$

Таким образом, в указанной ситуации $\ln \mu \sim \sqrt{\varepsilon}$ и согласно (1.1) величина $w$ значительно больше, чем $d$.

Оказывается, в случае мультипликаторов близких к единице постоянные $C_{1}$ и $C_{2}$ могут быть оценены в результате решения некоторых задач, не содержащих малого параметра. В частности, если $T$ удовлетворяет некоторому условию симметрии и выполняется одна хорошо известная гипотеза о свойствах стандартного отображения, то справедливо следующее равенство:

$$
\lim _{\varepsilon \rightarrow 0} \frac{w(\varepsilon) \ln \mu(\varepsilon)}{d(\varepsilon)}=\frac{4 \pi}{k_{0}},
$$


где

$$
\begin{gathered}
k_{0}=\inf \left\{k^{\prime}: \text { для любого } k>k^{\prime}\right. \text { стандартное отображение } \\
\left(\begin{array}{c}
J \\
\varphi
\end{array}\right) \mapsto\left(\begin{array}{l}
J \\
\psi
\end{array}\right)=\left(\begin{array}{c}
I+\frac{k}{2 \pi} \sin (2 \pi \varphi) \\
\varphi+J
\end{array}\right)
\end{gathered}
$$

не имеет инвариантной кривой, гомотопной окружности $I=0\}$.

Постоянная $k_{0}=0.971635 \ldots$ численно найдена в работах [3]-[5].

Пусть $\mathscr{A}$ - симплектическая площадь стохастического слоя и $\mathscr{A}_{\mathscr{D}}-$ площадь области $\mathscr{D}$. Тогда имеется следующий аналог соотношения (1.1):

$$
\mathscr{A} \sim \frac{\mathscr{A}_{\mathscr{D}} \ln \mathscr{A}_{\mathscr{D}}^{-1}}{\ln ^{2} \mu},
$$

а также аналог формулы (1.3) (при тех же предположениях):

$$
\lim _{\varepsilon \rightarrow 0} \frac{\mathscr{A} \ln ^{2} \mu}{\mathscr{A}_{\mathscr{D}} \ln \mathscr{A}_{\mathscr{D}}^{-1}}=\frac{8 \pi^{2}}{k_{0}} .
$$

В общем (несимметричном) случае функции

$$
\frac{w(\varepsilon) \ln \mu(\varepsilon)}{d(\varepsilon)}, \quad \frac{\mathscr{A}(\varepsilon) \ln ^{2} \mu(\varepsilon)}{\mathscr{A}_{\mathscr{D}}(\varepsilon) \ln \mathscr{A}_{\mathscr{D}}^{-1}(\varepsilon)}
$$

не имеют пределов при $\varepsilon \rightarrow 0$, а осциллируют между двумя постоянными.

В главе 7 мы сформулируем условия, при которых верны оценки (1.1)-(1.5). Соотношения (1.1) и (1.4) следуют из теоремы 7.2. Соотношения (1.3) и (1.5) вытекают из замечаний 7.11 и 7.12 при условии справедливости гипотезы 7.8 .

Частные случаи соотношения (1.1) были найдены физиками с помощью некоторых неформальных рассуждений в терминах критерия Чирикова [1], [6], [7], а также путем анализа сепаратрисного отображения. В [8] доказана оценка $w / d \leqslant$ const при условии $\ln \mu \sim 1$. В статье [9] при том же условии получена оценка $w / d \geqslant$ const. В. Ф. Лазуткин [10] получил оценку (1.1) для сепаратрис стандартного отображения.

\section{§ 2. Островки устойчивости в сепаратрисных лунках}

В главе 8 мы обсуждаем задачу о существовании эллиптических периодических траекторий, проходящих через сепаратрисные лунки, ограниченные отрезками устойчивой и неустойчивой сепаратрис гиперболической неподвижной точки. Мы покажем, что такие траектории, как правило, существуют в однопараметрических семействах симплектических отображений, близких к интегрируемым. Важно отметить, что эти траектории не порождаются какими бы то ни было периодическими траекториями невозмущенного отображения. Если $\varepsilon$ - параметр возмущения, то их период имеет порядок $\ln (1 /|\varepsilon|)$ при $\varepsilon \rightarrow 0$. 
Пусть $T_{\varepsilon}: N \rightarrow N$ - семейство симплектических отображений двумерной области $N$ на себя, $\varepsilon \in\left(-\varepsilon_{0}, \varepsilon_{0}\right)$ - параметр. Отображение $T_{0}$ предполагается интегрируемым, т.е. существует локально непостоянная функция $F: N \rightarrow \mathbb{R}$ такая, что $F \circ T_{0}=F$. Мы предполагаем, что $T_{0}$ имеет гиперболическую неподвижную точку $\sigma_{0}$, порождающую две петли сепаратрис (сепаратрисную "восьмерку") в $N$. Точка $\sigma_{0}$ является критической для первого интеграла $F$. Мы считаем эту критическую точку невырожденной.

При малых $\varepsilon \neq 0$ отображение $T_{\varepsilon}$ имеет гиперболическую неподвижную точку $\sigma_{\varepsilon}$, гладко зависящую от малого параметра. Я. Г. Синай [11] обнаружил, что эллиптические периодические траектории могут посещать сепаратрисные лунки, см. рис. 1.2. Далее мы называем такие траектории ЭПЛ (Эллиптические, Периодические, проходящие через Лунки). В примере, фигурирующем в [11], отображение далеко от интегрируемого.

Большое количество периодических решений около сепаратрис точки $\sigma_{\varepsilon}$ можно получить методами символической динамики, но все они неустойчивы. Можно рассуждать и так. Когда при изменении параметра асимптотические многообразия двигаются и приближаются к касанию, появляются пары периодических решений, каждая пара первоначально состоит из эллиптического и гиперболического решения. Впрочем, далее мы используем другие методы.

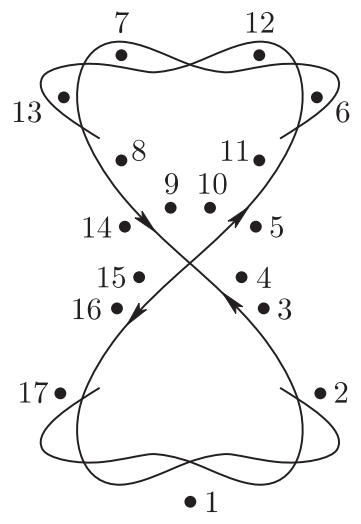

Рис. 1.2. Простейший тип периодической траектории, посещающей сепаратрисную лунку

В. В. Козлов показал, что условие расщепления сепаратрис в первом приближении по $\varepsilon$, следующее из теории Пуанкаре-Мельникова, влечет разрушение невозмущенных резонансных кривых, расположенных близко к невозмущенным сепаратрисам. В результате появляются невырожденные периодические решения [12]. (Многомерное обобщение этого наблюдения имеется в [13].) Все эти решения делают ровно один оборот внутри сепаратрисной петли. Некоторые из них устойчивы. Отметим, что при малых, но фиксированных $\varepsilon$ можно получить лишь конечное число таких траекторий. С. А. Довбыш [14] показал, что устойчивые периодические траектории, полученные таким образом, существуют только вне $\sqrt{|\varepsilon|}$-окрестности сепаратрис и, следовательно, не могут посещать сепаратрисные лунки, так как ширина последних имеет порядок $\varepsilon$. 
Каждой периодической траектории в окрестности сепаратрис можно сопоставить ее тип - число прохождений около точки $\sigma_{\varepsilon}$. Другими словами, траектория типа $n$ - это периодическая траектория сепаратрисного отображения периода $n$.

Простейший пример ЭПЛ-траектории приведен на рис. 1.2. Траектория вращается один раз внутри верхней петли сепаратрисной "восьмерки" и проходит один раз вдоль всей “восьмерки”. Согласно нашей классификации тип равен 3. Легко понять, что тип ЭПЛ-траектории всегда не меньше трех.

Пусть $\varepsilon_{0}>0$ достаточно мало и $J=J\left(T_{\varepsilon}\right) \subset\left(-\varepsilon_{0}, \varepsilon_{0}\right)-$ множество значений параметра $\varepsilon,|\varepsilon|<\varepsilon_{0}$, для которых отображение $T_{\varepsilon}$ имеет ЭПЛ-траекторию. Отображение $T_{0}$ будем считать фиксированным. Положим $T^{\prime}=d T_{\varepsilon} /\left.d \varepsilon\right|_{\varepsilon=0}$. Тогда $T^{\prime}$ - гамильтоново векторное поле.

Пусть $T_{\varepsilon}$ имеет гладкость $C^{\mathbf{r}}, \mathbf{r} \in\{13,14, \ldots, \infty, \omega\}$, и пусть $\mathscr{A}$ - пространство $C^{\mathbf{r}}$-гладких векторных полей на $N$. Снабдим $\mathscr{A} C^{2}$-топологией.

Tеорема 1.1. Существует открытое множество $\mathscr{S} \subset \mathscr{A}$ такое, ито для любого $T^{\prime} \in \mathscr{S}$ относительная мера множества $J$ на интервале $(-\varepsilon, \varepsilon)$ превосходит положительную постоянную, не зависящую от $\varepsilon \in\left(0, \varepsilon_{0}\right)$.

ГипотезА 1.2. Множество $\mathscr{S}$ можно выбрать плотным в $\mathscr{A}$.

Теорема 1.1 является следствием соответствующего результата для обратимых семейств $T_{\varepsilon}$.

Пусть $I_{\varepsilon}: N \rightarrow N$ есть $C^{\mathbf{r}}$-гладкое семейство инволюций $\left(I_{\varepsilon}^{2}=\mathrm{id}\right)$ таких, что $I_{\varepsilon}\left(\sigma_{\varepsilon}\right)=\sigma_{\varepsilon}$. Отображения $T_{\varepsilon}$ называются обратимыми относительно $I_{\varepsilon}$ (или $I_{\varepsilon}$-обратимыми), если

$$
T_{\varepsilon} \circ I_{\varepsilon}=I_{\varepsilon} \circ T_{\varepsilon}^{-1} .
$$

Если $T_{0}$ обратимо, то $I_{0}$ отображает сепаратрисную "восьмерку" на себя. Мы будем предполагать, что $I_{0}$ сохраняет петли "восьмерки", т.е. отображает каждую из них на себя. Такая инволюция действует как симметрия относительно вертикальной оси симметрии "восьмерки".

Теорема 1.3. Предположим, что $T_{\varepsilon}$ обратимо относительно семейства инволючий $I_{\varepsilon}$, сохраняющих петли, и, по крайней мере, одна пара сепаратрис точки $\sigma_{\varepsilon}$ расщепляется в первом приближении по $\varepsilon$. Тогда относительная мера множества $J$ на интервале $(-\varepsilon, \varepsilon)$ превосходит некоторую положительную постоянную, не зависящую от $\varepsilon \in\left(0, \varepsilon_{0}\right)$.

Эллиптические периодические траектории, как правило, порождают островки устойчивости. Мы покажем, что площадь такого островка для типичной ЭПЛ-траектории имеет тот же порядок, что и площадь сепаратрисной лунки. Это означает, что, в принципе, эти островки можно наблюдать на картинках, полученных в результате численной симуляции динамики. Однако наивные попытки наблюдать островки устойчивости в сепаратрисных лунках обычно оканчиваются неудачей. Для этого имеется две причины. Во-первых, мера множества $J$, соответствующего "не очень маленьким" островкам, невелика, хотя это сильно зависит от параметров отображения. Если $\mu_{0}$ обозначает старший мультипликатор $T_{0}$ в точке $\sigma_{0}$, то грубые численные оценки показывают, 
что эта мера изменяется от 0.004 до 0.3 , когда $\mu_{0}$ меняется от 1.1 до 10. Вовторых, средняя относительная мера "не очень маленького" острова в лунке обычно меняется между $10^{-8}$ и $10^{-4}$ в том же промежутке $\mu_{0}$. Более подробную информацию о численном исследовании задачи см. в [15].

\section{§ 3. Диффузия Арнольда: постановка проблемы}

Дальнейшие применения сепаратрисного отображения, рассматриваемые в этой статье, связаны с задачей об эволюции медленных переменных в гамильтоновых системах. Прежде чем сформулировать основные результаты в этом направлении, опишем, в чем состоит задача.

В [16] предложен пример гамильтоновой системы вида

$$
\begin{gathered}
\dot{x}=\partial H / \partial y, \quad \dot{y}=-\partial H / \partial x, \quad H=H_{0}(y)+\varepsilon H_{1}(x, y, t, \varepsilon), \\
x \in \mathbb{T}^{n}=\mathbb{R}^{n} / \mathbb{Z}^{n}, \quad y \in \mathbb{R}^{n}, \quad t \in \mathbb{T}, \quad \varepsilon \in\left(-\varepsilon_{0}, \varepsilon_{0}\right),
\end{gathered}
$$

с $n=2$ и выпуклым по действиям $y$ невозмущенным гамильтонианом $H_{0}$, в которой переменные $y$ могут измениться вдоль траектории на величину порядка единицы. Позднее это явление получило название диффузии Арнольда. В [16] был также предложен механизм, порождающий такую диффузию. В возмущенной задаче должен существовать набор гиперболических торов (переходная цепочка). Соответствующая цепочка устойчивых и неустойчивых асимптотических поверхностей должна быть соединена гетероклиническими траекториями. Тогда на траектории, идущей вдоль этой цепочки, имеет место дрейф переменных действие.

Основной вопрос, связанный с диффузией Арнольда в системах (1.6) звучит так. Является ли диффузия типичным свойством? При этом важно, в каком классе гладкости лежит $H$. Обычно наиболее интересным считается вещественно-аналитический случай. Он же является технически наиболее сложным.

Согласно теории Нехорошева [17], в вещественно-аналитических системах, удовлетворяющих довольно слабым условиям (так называемым условиям крутизны), средняя скорость дрейфа действий вдоль траектории оценивается сверху экспоненциально малой величиной $e^{-\alpha \varepsilon^{-\beta}}$ с положительными $\alpha, \beta$. Экспоненциально малые эффекты представляют основную трудность для анализа диффузии. Другая трудность связана с тем фактом, что торы в переходной цепочке образуют, как правило, не непрерывное, а канторово семейство. Оно содержит дыры. Если эти дыры велики, то асимптотическим поверхностям двух торов, разделенных дырой, трудно дотянуться друг до друга. Оценки ширины дыр используют теорию КАМ и поэтому весьма громоздки.

При построении своего примера Арнольд не преодолел этих трудностей, а обошел их с помощью некоторых трюков. В примере Арнольда переходная цепочка образована гладким семейством гиперболических торов. С экспоненциально малыми эффектами удается справиться благодаря тому, что возмущение зависит от двух малых параметров: $\varepsilon$ и $\delta \sim e^{-c / \sqrt{\varepsilon}}, c>0$. Параметр $\varepsilon$ несет ответственность за появление гиперболических торов, а $\delta$ - за существование гетероклинических связей между ними. Так как $\delta$ экспоненциально мал 
относительно $\varepsilon$, то существование этих связей можно установить с помощью метода Пуанкаре-Мельникова.

Р. Дуади [18] построил $C^{\infty}$-гладкие гамильтоновы системы с тремя степенями свободы, имеющие формально устойчивые, но неустойчивые по Ляпунову эллиптические положения равновесия, причем неустойчивость порождается диффузией Арнольда. Более гладкая (Жевре- $\alpha, \alpha>1)$ ситуация рассмотрена в [19]. В [19] также содержится вариант теории Нехорошева для квазивыпуклых гамильтоновых систем класса Жевре.

В [20] в духе идей Арнольда построен широкий класс вещественно-аналитических систем (весьма вырожденных) с диффузионными траекториями. Возмущения, зависящие от двух малых параметров, рассмотрены в [21]. В отличие от исходного примера Арнольда второй параметр $\delta$ не экспоненциально мал относительно $\varepsilon$, а имеет вид $\delta=\varepsilon^{p}$, где постоянная $p$ достаточно велика. Вариационный подход в примере Арнольда используется в [22]. В [23] произведен численный анализ примера Арнольда с $\varepsilon=\delta$.

Дж. Н. Мезер [24] анонсировал вариационное доказательство типичности диффузии Арнольда в системах (1.6) с положительно определенным гессианом $\partial^{2} H_{0} / \partial y^{2}$ в случае двух с половиной степеней свободы. Однако полного доказательства пока не появилось.

Существует несколько более простых задач, где явления типа диффузии (т.е. дрейф медленных переменных вдоль переходных цепочек) происходят без экспоненциально малых эффектов. К ним относятся задача Мезера и априори неустойчивые системы. Ниже мы рассмотрим эти два класса систем подробнее. Следует отметить, что с помощью метода сепаратрисного отображения в таких системах удается не только доказывать существование диффузионных траекторий, но и оценивать максимальную скорость дрейфа медленных переменных.

\section{§4. Диффузия в задаче Мезера}

В главах 5 и 9 мы рассматриваем следующую гамильтонову систему:

$$
\begin{aligned}
& \dot{q}=\partial H / \partial p, \dot{p}=-\partial H / \partial q, \\
& H(q, p, t)=T(q, p)+V(q, t), \quad q \in M^{2}, \quad p \in T_{q}^{*} M^{2}, \quad t \in \mathbb{T},
\end{aligned}
$$

где $M^{2}$ - гладкое компактное двумерное многообразие, $T(q, p)$ - кинетическая энергия (риманова метрика на $\left.M^{2}\right), V(q, t)$ - периодическая по времени потенциальная энергия.

Система неавтономна, и энергия может изменяться на решениях.

Теорема 1.4 (Дж. Н. Мезер). Пусть род $M^{2}$ положителен. Тогда для типичных $T$ и $V$ существует решение $\eta(t)$ системы (1.7)-(1.8), на котором $H(\eta(t), t) \rightarrow+\infty$ nрu $t \rightarrow+\infty$.

Условия Мезера типичности метрики $T$ и потенциала $V$ сформулированы в 1 главы 9. С помощью метода сепаратрисного отображения в главе 9 мы получим еще одно доказательство теоремы 1.4 и оценим максимально возможную скорость роста энергии: 
Теорема 1.5 [25]. При условиях Мезера существует решение $\eta(t)$ системы (1.7)-(1.8) такое, что при всех $t \geqslant 0$

$$
H(\eta(t), t) \geqslant A^{-} t+B^{-}
$$

для некоторьх констант $A^{-}>0 u B^{-}$.

Поскольку $d H / d t=\partial V / \partial t \leqslant A^{+}$, то на любом решении $H(t) \leqslant A^{+} t+B^{+}$, $A^{+}, B^{+}=$const. Таким образом, рост энергии более быстрый, чем линейный по времени, невозможен ни на каком решении. В этом смысле оценка (1.9) точна.

В задаче Мезера отсутствует малый параметр $\varepsilon$, который обычно имеется в задачах о диффузии медленных переменных. Однако он появляется как отношение $V / T$ при условии, что полная энергия достаточно велика. Действительно, потенциальная энергия ограничена. Поэтому рост полной энергии происходит за счет возрастания кинетической энергии. Система (1.7)-(1.8) при больших $H$ представляет собой возмущение геодезического потока (т.е. системы с гамильтонианом $T$ ) неавтономным потенциалом $V$. При этом роль медленной переменной играет сам гамильтониан $H$.

Геометрический механизм роста энергии на решении $\eta(t)$ состоит в следующем. Пусть $\sigma$ - минимальная геодезическая (относительно метрики $T$ ) в некотором гомотопическом классе замкнутых кривых на $M^{2}$. Хорошо известно, что в общем случае эта геодезическая гиперболична, т.е. экспоненциально неустойчива относительно геодезического потока. Так как возмущение $V$ неавтономно, время естественно считать фазовой переменной. Тогда $\sigma$ порождает в невозмущенной системе с гамильтонианом $T$ однопараметрическое семейство гиперболических двумерных торов. Проекцией каждого из этих торов на пространство положений $M^{2} \times \mathbb{T}_{t}$ является $\sigma \times \mathbb{T}_{t}$, где $\mathbb{T}_{t}$ - окружность, соответствующая времени. Семейство параметризовано значением гамильтониана $T$. Большинство из торов для достаточно больших $T$ выживают при возмущении, при этом появляются гетероклинические связи между их асимптотическими многообразиями и, следовательно, переходные цепочки. Соответствующие доказательства, использующие методы теории КАМ, имеются в [26] и [27]. Отметим, что исходное доказательство Мезера, основанное на вариационных методах, до сих пор не опубликовано. Доказательство, частично использующее вариационные идеи Мезера, содержится в [28]. В работах [26], [29] получены различные многомерные обобщения этого результата. Результат, аналогичный теореме 1.5 , анонсировал Р. де ла Яве.

В главе 5 мы представим формулы для сепаратрисного отображения, которое описывает динамику в окрестности указанных выше гетероклинических связей. Сепаратрисное отображение задачи Мезера представляет собой возмущение по малому параметру $V / T$ сепаратрисного отображения невозмущенной системы. В невозмущенной системе оно представляет собой прямое произведение сепаратрисного отображения Шильникова (глава 4), которое имеет инвариантное гиперболическое множество, и тождественного (сохранение энергии). Таким образом получаем возмущение частично гиперболической системы. Следовательно, сепаратрисное отображение возмущенной системы имеет много хаотических траекторий. В главе 9 мы построим эти траектории, используя идею 
метода антиинтегрируемого предела - построения сжимающего оператора на пространстве траекторий. Для доказательства теоремы 1.5 необходимо выбрать среди построенных траекторий траектории с максимально возможным ростом энергии, что можно сделать, используя явные формулы сепаратрисного отображения.

\section{§ 5. Диффузия в априори неустойчивых системах}

Объектом исследования в главах 6,10 и 11 являются так называемые априори неустойчивые гамильтоновы системы. Эти системы были введены в [30] с целью уйти от одной из главных технических трудностей при исследовании диффузии Арнольда - экспоненциально малых эффектов. Априори неустойчивая система является возмущением интегрируемой. Изучается динамика в окрестности гиперболического многообразия, образованного семейством невозмущенных (частично) гиперболических торов. Существование такого семейства заложено в определении априори неустойчивой системы. В главе 6 мы строим многомерный аналог сепаратрисного отображения Заславского. Полученные формулы являются далее основой для исследования проблемы диффузии Арнольда в таких системах.

Мы рассматриваем лишь случай, когда размерность гиперболических торов максимальна. Как обычно, удобнее рассматривать неавтономную систему с периодической по времени функцией Гамильтона. Если число степеней свободы равно $n+1+1 / 2$, то размерность гиперболических торов равна $n+1$. В невозмущенной (интегрируемой) системе эти торы образуют $n$-параметрическое семейство, являющееся $(2 n+1)$-мерным гиперболическим множеством. Сепаратрисное отображение задает динамику в окрестности асимптотических многообразий этого множества. Сепаратрисное отображение с одинаковым успехом работает около нерезонансных и резонансных торов. При этом слова "гиперболические торы" и "асимптотические многообразия" означают объекты невозмущенной системы. Мы не используем тот факт, что некоторые из них выживают после возмущения.

Рассмотрим гамильтонову систему с гамильтонианом

$$
H(y, x, v, u, t, \varepsilon)=H_{0}(y, v, u)+\varepsilon H_{1}(y, x, v, u, t)+O\left(\varepsilon^{2}\right) .
$$

Здесь $x=\left(x_{1}, \ldots, x_{n}\right) \bmod 1$ - точка $n$-мерного тора $\mathbb{T}^{n}, y=\left(y_{1}, \ldots, y_{n}\right) \in \mathscr{D}_{0} \subset$ $\mathbb{R}^{n}$, где $\mathscr{D}_{0}$ - открытая область, и $(v, u)$ - точка области $D \subset \mathbb{R}^{2}$. Пары переменных $x$ и $y, u$ и $v$ канонически сопряжены, т.е. симплектическая структура и уравнения Гамильтона имеют вид $\omega=d y \wedge d x+d v \wedge d u$,

$$
\dot{y}=-\partial H / \partial x, \quad \dot{x}=\partial H / \partial y, \quad \dot{v}=-\partial H / \partial u, \quad \dot{u}=\partial H / \partial v .
$$

Функция $H$ считается 1-периодической по $t$, а параметр $\varepsilon$ - малым. Удобно считать $t$ точкой тора $\mathbb{T}^{1}$.

Система с гамильтонианом $H_{0}$ интегрируема и называется невозмущенной. Далее мы предполагаем, что гамильтониан (1.10) удовлетворяет нескольким условиям. 
$\mathbf{H}_{\mathbf{0}}$ 1. Функиия $H C^{\mathbf{r}}$-гладкая по $(y, x, v, u, t, \varepsilon)$, где $\mathbf{r}$ достаточно велико. ${ }^{1}$

$\mathbf{H}_{\mathbf{0}}$ 2. Для любого $y^{0} \in \mathscr{D}_{0}$ функция $H_{0}\left(y^{0}, v, u\right)$ имеет невырожденную седловую точку $(v, u)=\left(v^{0}, u^{0}\right)$. Любая точка $\left(v^{0}, u^{0}\right)$ лежит в компактной компоненте связности множества $\left\{(v, u) \in D: H_{0}\left(y^{0}, v, u\right)=H_{0}\left(y^{0}, v^{0}, u^{0}\right)\right\}$. Более того, $\left(v^{0}, u^{0}\right)$ - единственная критическая точка $H_{0}\left(y^{0}, v, u\right)$ на этой компоненте.

Точка $\left(v^{0}, u^{0}\right) \in D$ гладко зависит от $y^{0}$ и является гиперболическим положением равновесия системы с одной степенью свободы и гамильтонианом $H_{0}\left(y^{0}, v, u\right)$. Соответствующие сепаратрисы сдвоены и образуют кривую типа восьмерки. Петли "восьмерки" далее обозначаются $\widehat{\gamma}^{ \pm}\left(y^{0}\right)$, где $\widehat{\gamma}^{+}\left(y^{0}\right)$ называется верхней петлей, а $\widehat{\gamma}^{-}\left(y^{0}\right)$ - нижней. Петли $\widehat{\gamma}^{ \pm}\left(y^{0}\right)$ имеют естественную ориентацию, порожденную потоком системы. Ориентация на $D$ определяется системой координат $v, u$.

$\mathbf{H}_{\mathbf{0}}$ 3. При всех $y^{0} \in \mathscr{D}_{0}$ естественная ориентация $\widehat{\gamma}^{ \pm}\left(y^{0}\right)$ совпадает с ориентацией области $D$, т.е. движение по сепаратрисам происходит против часовой стрелки (см. рис 1.3).

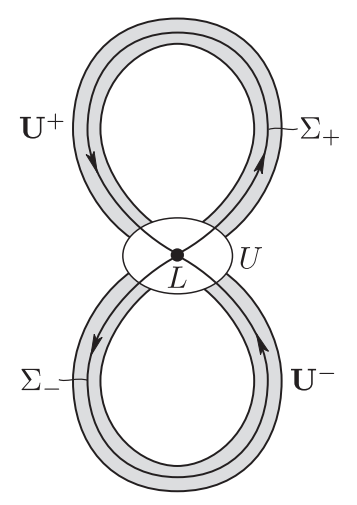

Рис. 1.3. Множества $U$ и $\mathbf{U}^{ \pm}$в случае $n=0$

Это условие, очевидно, ограничительным не является.

$\mathbf{H}_{\mathbf{0}}$ 4. В невозмущенном гамильтониане переменные у отделены от $и$ u $v$ (m.e. $\left.H_{0}(y, v, u)=F(y, f(v, u))\right)$.

Условие $\mathbf{H}_{\mathbf{0}} \mathbf{4}$, по-видимому, не обязательно включать в определение априори неустойчивой системы. В некоторых конструкциях без него можно обойтись (см., например, [31]). Согласно $\mathbf{H}_{0} 4$ положения равновесия $\left(v^{0}, u^{0}\right)$ не зависят от $y^{0}$. Будем считать, что $\left(v^{0}, u^{0}\right)=0$.

Сепаратрисное отображение, описывающее динамику системы (1.10)-(1.11) при условиях $\mathbf{H}_{\mathbf{0}} \mathbf{1}-\mathbf{H}_{\mathbf{0}} \mathbf{4}$ в окрестности семейства гиперболических торов

$$
N\left(y^{0}\right)=\left\{(y, x, v, u, t): y=y^{0}, v=u=0\right\}
$$

и их асимптотических многообразий, строится в главе 6. Далее оно применяется к проблеме диффузии в простейшей нетривиальной ситуации - в случае

\footnotetext{
1 Заведомо годится $\mathbf{r} \geqslant 13$.
} 
двух с половиной степеней свободы. Чтобы сформулировать соответствующий результат, положим $n=1$. Тогда $\mathscr{D}_{0} \subset \mathbb{R}-$ интервал. Положим также

$$
E(y)=H_{0}(y, 0,0), \quad \nu=E_{y}: \mathscr{D}_{0} \rightarrow \mathbb{R} .
$$

Следующее условие зависит от положительного параметра $c_{*}$.

$\mathbf{H}_{\mathbf{0}} \mathbf{5}\left(c_{*}\right)$. Функция $\nu^{\prime}(y)=d \nu(y) / d y$ не обращается в нуль в сильных резонансах, т.е. в точках, где $\nu=p / q, p \in \mathbb{Z}, q \in \mathbb{N}, q<1 / c_{*}$. Более того, для любого у $\in \mathscr{D}_{0}$ имеем: $\left|\nu^{\prime}(y)\right|+\left|\nu^{\prime \prime}(y)\right|>2 c_{\nu}$ с некоторым $c_{\nu}>0$.

Пусть $\mathscr{D} \subset \mathscr{D}_{0}$ - интервал такой, что его замыкание $\overline{\mathscr{D}}$ также лежит в $\mathscr{D}_{0}$.

Теорема 1.6 [32]. Предположим, что $H_{0}$ удовлетворяет $\mathbf{H}_{\mathbf{0}} \mathbf{1}-\mathbf{H}_{\mathbf{0}} \mathbf{4}$. Тогда для типичного $H_{1}$ существуют $c_{*}, \varepsilon_{0}>0$ такие, что если $\mathbf{H}_{\mathbf{0}} \mathbf{5}\left(c_{*}\right)$ выполнено $u \varepsilon \in\left(0, \varepsilon_{0}\right)$, то возмущенная система имеет решение

$$
(x(t), y(t), v(t), u(t)), \quad t \in[0, T],
$$

со следующими свойствами:

(i) $\overline{\mathscr{D}} \subset[y(0), y(T)]$,

(ii) $c_{1}<\frac{\varepsilon}{|\ln \varepsilon|} T<c_{2}$ для некоторых $c_{1}, c_{2}>0$.

Замечания. 1. Слова “типичный $H_{1}$ " означают, что $H_{1}$ принадлежит открытому плотному множеству в $C^{\mathbf{r}}\left(\mathscr{D}_{0} \times \mathbb{T} \times D \times \mathbb{T}, \mathbb{R}\right)$. Точнее, $H_{1}$ должен удовлетворять условиям $\mathbf{H}_{\mathbf{1}} \mathbf{1}-\mathbf{H}_{\mathbf{1}} \mathbf{3}$ (см. § 2 главы 11). Условия $\mathbf{H}_{\mathbf{1}} \mathbf{1}-\mathbf{H}_{\mathbf{1}} \mathbf{3}$ конструктивны, и, в принципе, их можно проверить для данного $H_{1}$.

Теорему 1.6 можно сформулировать несколько иначе. Положив $\varepsilon=1$, можно сказать, что $H_{1}$ достаточно мал в $C^{\mathbf{r}}$-норме. Но тогда в качестве условия типичности для $H_{1}$ следует говорить, что $H_{1}$ лежит в так называемом подмножестве типа дополнения к остриям (cusp-residual subset) малого шара $C^{\mathbf{r}}$ (cp. [24]). Эти две возможности, очевидно, эквивалентны.

2. Утверждение (i) означает, что (1.13) - это диффузионная траектория, проходящая через $\overline{\mathscr{D}}$, и, согласно (ii), средняя скорость диффузии имеет порядок $\varepsilon /|\ln \varepsilon|$. Та же оценка для скорости диффузии была получена в [33] для квазипериодического возмущения маятника, в [34] при дополнительном предположении, что имеется переходная цепочка с хорошими свойствами, и в [35], [36] в "нерезонансной" области многомерного априори неустойчивого варианта примера Арнольда. Отметим, что в указанных статьях возмущения являются тригонометрическими полиномами и, в частности, нетипичны.

3. Вариационный подход в задаче диффузии в априори неустойчивых системах с $2+1 / 2$ степенями свободы развивался в [37] и доведен до полных доказательств типичности диффузии в [38]. Более традиционные геометрические методы в композиции с новыми идеями в окрестности резонансов использованы в [39], [40]. В этих работах максимальная скорость диффузии не оценивалась.

4. Теорема 1.6 остается верной, если в уравнениях (1.11) имеются неконсервативные добавки порядка $O\left(\varepsilon^{2}\right)$.

5. На самом деле теорема 1.6 дает существование бесконечного количества траекторий со свойствами (i)-(ii). Очевидная оценка снизу для меры $\mu$ соответствующих начальных условий $\mu \geqslant e^{-c T}, c>0$, по-видимому, достаточно реалистична в том смысле, что оценка сверху должна быть аналогичной. 
6. В наших конструкциях нигде не используется существование гиперболических торов в возмущенной системе. Поэтому, формально говоря, у нас отсутствует "проблема больших дыр" ("large gap problem"), т.е. проблема построения гетероклинических связей между двумя гиперболическими торами, расположенными по разные стороны от сильного резонанса (подробное обсуждение этой проблемы и некоторых подходов к ее решению см. в [39], [40]). Впрочем, эта проблема полностью не исчезает. Ее следы (в других терминах) можно найти в части главы 11 , касающейся пересечения сильных резонансов.

Используемые методы примыкают по духу к методам символической динамики. Мы строим коды, которые заставляют двигаться траекторию в данном направлении. Согласно лемме о присоединении (§2 главы 10) код порождает траекторию. Выбор кода имеет серьезные ограничения. В нерезонансной области хороший код легко строится. При прохождении сильных резонансов ситуация более трудная: иногда даже приходится в течение некоторого времени двигаться в противоположном направлении вдоль оси $y$, но в конце концов удается обеспечить эволюцию в нужном направлении со средней скоростью порядка $\varepsilon / \ln \varepsilon$.

7. Аналог теоремы 1.6 должен иметь место и в случае произвольного $n$. Ожидаемый эффект состоит в следующем. Для типичной априори неустойчивой системы в малой окрестности произвольной гладкой кривой $\gamma \subset \mathscr{D}_{0}$ должны существовать решения, идущие вдоль $\gamma$ со средней скоростью порядка $\varepsilon / \ln \varepsilon$.

ПримеР. Рассмотрим систему (1.11) с $H=H_{0}+\varepsilon H_{1}$,

$$
\begin{gathered}
H_{0}=\frac{y^{2}}{2}+\frac{v^{2}}{2}+\Omega^{2} \cos (2 \pi u), \quad H_{1}=\cos (2 \pi u) f(x, t), \\
f(x, t)=a(\cos (2 \pi x)+\cos (2 \pi t)+b \cos (2 \pi(x-t))+b \cos (2 \pi(x+t))) .
\end{gathered}
$$

Невозмущенная система является прямым произведением ротатора на цилиндре $Z_{x y}=\{x \bmod 1, y\}$ и маятника на цилиндре $Z_{u v}=\{u \bmod 1, v\}$.

Положим $\mathscr{D}_{0}=(-3 / 2,3 / 2)$. Так как мы интересуемся динамикой около сепаратрис маятника, будем считать, что $D \subset Z_{u v}$ - окрестность сепаратрис. Тогда $D$ гомеоморфна окрестности восьмерки на плоскости. Невозмущенный гамильтониан $H_{0}$ удовлетворяет условиям $\mathbf{H}_{\mathbf{0}} \mathbf{1}-\mathbf{H}_{\mathbf{0}} \mathbf{5}\left(c_{*}\right)$ с произвольным $c_{*}$.

Далее для удобства полагаем

$$
\Omega=\pi / 2, \quad a=\pi^{2} / 8
$$

ТеОрема 1.7. Существует положительная постоянная $b_{0}$ такая, что для любого $b \in\left(0, b_{0}\right)$ возмущение $H_{1}$ типично в смысле, требуемом в теореме 1.6 .

СледСтвиЕ 1.8. Предположим, что $b \in\left(0, b_{0}\right) u \beta \in(0,3 / 2)$. Тогда при достаточно малых $\varepsilon>0$ система (1.11), (1.14) имеет решение, у которого у-компонента проходит через интервал $(-\beta, \beta)$ в течение времени порядка $\ln \varepsilon / \varepsilon$. 


\section{Глава 2. Предварительные замечания}

В этой главе мы зафиксируем некоторые термины, обозначения и сформулируем хорошо известные факты, используемые далее.

\section{§ 1. Термины и обозначения}

Далее всюду $c$ обозначает постоянные, не зависящие от $\varepsilon$ и не играющие существенной роли в наших построениях. Эти постоянные считаются положительными и достаточно малыми. Если нижний индекс у функции является вещественной переменной, то он обозначает частную производную. Например, $\Theta_{\xi} \equiv \partial \Theta / \partial \xi$. Скобки $\langle\cdot, \cdot\rangle$ обозначают стандартное скалярное произведение в $\mathbb{R}^{n}$. Гладкими функциями будем называть функции класса $C^{\mathbf{r}}$, где $\mathbf{r}$ достаточно велико. Точнее, в обозначении класса гладкости $C^{\mathbf{r}}$ предполагается, что $\mathbf{r}$ может принимать произвольное значение из множества $\{9,10, \ldots, \infty, \omega\}$. Пусть $f$ и $g$ - гладкие функции. Будем говорить, что $f=O_{k}(g)$, если $f=h g^{k}$, где $h$ - некоторая гладкая функция.

Автономную гамильтонову систему с $2 n$-мерным фазовым пространством (симплектическим многообразием) будем называть системой с $n$ степенями свободы. Если же функция Гамильтона зависит от времени, причем эта зависимость периодическая, то будем говорить, что система имеет $n+\frac{1}{2}$ степеней свободы.

Пусть $T^{t}: M \rightarrow M$ - однопараметрическая группа диффеоморфизмов риманова многообразия $(M$, dist $), t \in \mathbb{Z}$ или $\mathbb{R}$. Орбита $\gamma(t)=T^{t}(\gamma(0))$ называется гетероклинической к инвариантным относительно $T^{t}$ множествам $N_{-} \subset M$ и $N_{+} \subset M$, если $\operatorname{dist}\left(\gamma(t), N_{ \pm}\right) \rightarrow 0$ при $t \rightarrow \pm \infty$. Если $N_{-}=N_{+}$, то $\gamma(t)$ называется гомоклинической к этому множеству.

\section{§ 2. Неподвижные точки двумерных симплектоморфизмов}

Диффеоморфизм $T$ двумерной области $D \subset \mathbb{R}_{z=\left(z_{1}, z_{2}\right)}^{2}$ на себя называется симплектическим (симплектоморфизмом), если он сохраняет форму площади $\omega^{2}=d z_{2} \wedge d z_{1}$. Пусть $\sigma$ - неподвижная точка симплектоморфизма $T: T(\sigma)=\sigma$. Мы будем исследовать динамику в окрестности этой точки.

$\mathrm{K}$ указанной задаче сводится исследование отображения $T$ в окрестности периодического решения $\sigma \mapsto T(\sigma) \mapsto \cdots \mapsto T^{n}(\sigma)=\sigma$ (с помощью замены $T$ на $\left.T^{n}\right)$ и исследование гамильтоновой системы с полутора или двумя степенями свободы в окрестности периодического решения (с помощью перехода к отображению Пуанкаре).

Свойства динамической устойчивости неподвижной точки в значительной степени определяются матрицей монодромии $M=\partial T /\left.\partial z\right|_{z=\sigma}$, так как в линейном приближении отображение $T$ есть умножение на $M$. Собственные значения матрицы монодромии называются мультипликаторами неподвижной точки $\sigma$. Так как $T$ сохраняет площадь, $\operatorname{det} M=1$. Следовательно, характеристический полином для $M$ представляется в виде $\mu^{2}-\mu \operatorname{tr} M+1$. Имеется четыре возможности: 
- $|\operatorname{tr} M|>2$. Мультипликаторы - вещественны и различны. Неподвижная точка называется гиперболической.

- $|\operatorname{tr} M|<2$. Мультипликаторы равны по модулю единице и различны. Точка $\sigma$ называется эллиптической.

$-\operatorname{tr} M=-2$. Мультипликаторы равны -1 . Точка $\sigma$ называется параболической.

$-\operatorname{tr} M=2$. Мультипликаторы равны 1 . Точка $\sigma$ называется вырожденной.

При малом возмущении системы невырожденные неподвижные точки не исчезают, а лишь слегка деформируются.

\section{§ 3. Нормальные координаты в окрестности гиперболической неподвижной точки}

Рассмотрим особо гиперболические неподвижные точки и периодические решения. Будем считать, что соответствующие мультипликаторы положительны (случай отрицательных мультипликаторов сводится к указанному путем рассмотрения $\sigma$ как неподвижной точки отображения $T^{2}$ ). Одним из замечательных свойств симплектоморфизма в окрестности такого решения является существование так называемых нормальных координат. А именно, верна следующая теорема.

Теорема 2.1. Пусть $\sigma$ - гиперболическая неподвижная точка гладкого симплектического отображения $T$. Тогда в окрестности точки $\sigma$ существуют гладкие симплектические координаты $(q, p)$ и функиия $\mathscr{M}(q p)$ такие, что $\sigma=(0,0)$ и в новых координатах отображение $T$ имеет вид

$$
(q, p) \mapsto(q \mathscr{M}(q p), p / \mathscr{M}(q p)),
$$

где $\mu=\mathscr{M}(0)>1 u \mu^{-1}-$ мулътипликаторь $\sigma$.

ЗАмечАниЕ 2.2. 1) Координаты, определенные в теореме 2.1, называются нормальными. Существование преобразования к нормальным координатам на формальном уровне установлено Г. Д. Биркгофом [41]. Сходимость преобразования в аналитическом случае доказана Ю. Мозером [42]. Случай конечной гладкости рассмотрен в [43].

2) Гладкость нормальных координат и функции $\mathscr{M}$ зависит линейно от гладкости $T$. Из [43] следует, что если $T$ класса $C^{\mathbf{r}}$, то отображение, задающее замену координат, можно взять $C^{s}$-гладким, где $s \leqslant(\mathbf{r}-3) / 2$. В частности, если $\mathbf{r} \geqslant 13$, то существуют $C^{5}$-гладкие нормальные координаты.

3) Кривые $\Gamma^{s}=\{(q, p): q=0\}$ и $\Gamma^{u}=\{(q, p): p=0\}$ являются инвариантными для системы (2.1). Любое решение, расположенное на $\Gamma^{s}$ (соответственно на $\Gamma^{u}$ ) экспоненциально стремится к $\sigma$ при $n \rightarrow+\infty$ (соответственно при $n \rightarrow-\infty)$. Кривые $\Gamma^{s, u}$ называются асимптотическими к $\sigma$ или сепаратрисами.

4) Нормальные координаты $(q, p)$ определены неоднозначно. Например, координаты $\left(q^{\prime}, p^{\prime}\right)=(-p, q)$ - нормальные. Более того, для любой гладкой функции $r(z)(r(0) \neq 0)$ координаты $q^{\prime}=q / r(q p), p^{\prime}=p r(q p)$ также являются нормальными. 
Пусть имеется гамильтонова система с полутора степенями свободы с фазовым пространством $D \subset \mathbb{R}^{2}$ и гамильтонианом $H(z, t), z \in D, t \in \mathbb{T}=\mathbb{R} / \mathbb{Z}$. Аналогом теоремы 2.1 является следующая теорема.

Теорема 2.3. Пусть $(\sigma(t), t)$ - гиперболическое периодическое решение гамильтоновой системы с гамильтонианом $H, \sigma(0)=\sigma(1)$. Тогда на расширенном фазовом пространстве $D \times \mathbb{T}_{t}$ в окрестности кривой $(\sigma(t), t)$ существуют гладкие симплектические координаты $(q, p, t)$ такие, что

1) время $t$ совпадает с исходным,

2) периодическое решение $(\sigma(t), t)=(q=0, p=0, t)$,

3) в новых координатах гамильтониан зависит только от произведения qр: $H=\mathscr{H}(p q)$.

\section{Глава 3. Сепаратрисное отображение Заславского}

В этой главе мы получим формулы сепаратрисного отображения для двумерных симплектических отображений и гамильтоновых систем с полутора степенями свободы, близких к интегрируемым.

\section{§ 1. Определение и формулы}

1.1. Двумерный симплектоморфизм. Рассмотрим семейство симплектических отображений $T_{\varepsilon}$, определенных на двумерной области $D \subset \mathbb{R}_{z=\left(z_{1}, z_{2}\right)}$, зависящее от малого параметра $\varepsilon,|\varepsilon|<\varepsilon_{0}$. Предположим, что

I. Отображение $T_{0}$ имеет гиперболическую неподвижную точку $\sigma$;

II. $T_{0}$ интегрируемо, т.е. существует гладкая локально непостоянная функция $F: D \rightarrow \mathbb{R}$ такая, что $F \circ T_{0}=F$.

Таким образом, динамика невозмущенного отображения $T_{0}$ проста. Область $D$ расслаивается на инвариантные кривые уровня интеграла $F$. Гиперболическая неподвижная точка порождает четыре асимптотические ветви (сепаратрисы) $\Gamma^{u \pm}$ и $\Gamma^{s \pm}$ - две неустойчивые и две устойчивые соответственно (см. рис. 3.1). Сепаратрисы лежат на одном уровне интеграла $F$. Как правило, в интегрируемых системах неустойчивая и устойчивая сепаратрисы совпадают. А именно, пусть

III. $\Gamma^{s \pm}=\Gamma^{u \pm}=: \Gamma^{ \pm}$, так что сепаратрисы $\Gamma^{+}, \Gamma^{-}$и точка $\sigma$ образуют фигуру типа восьмерки (см. рис. 3.1).

Кривые $\Gamma^{+}$и $\Gamma^{-}$будем условно называть верхней и нижней петлей “восьмерки" соответственно. Без ограничения общности можно считать, что $F(\sigma)=0$. Легко установить, что $\left.d F\right|_{z=\sigma}=0$. Далее считаем, что $\sigma-$ невырожденная критическая точка $F$, т.е.

IV. $\operatorname{det} \frac{\partial^{2} F}{\partial z^{2}}(\sigma) \neq 0$.

Обозначим через $\mu>1$ мультипликатор неподвижной точки $\sigma$ и $\lambda=\ln \mu{ }^{2}$

\footnotetext{
${ }^{2}$ Если $T_{0}$ - отображение за период, равный 1 , гамильтоновой системы с полутора степенями свободы, то $\lambda$ - показатель Ляпунова соответствующего периодического решения.
} 

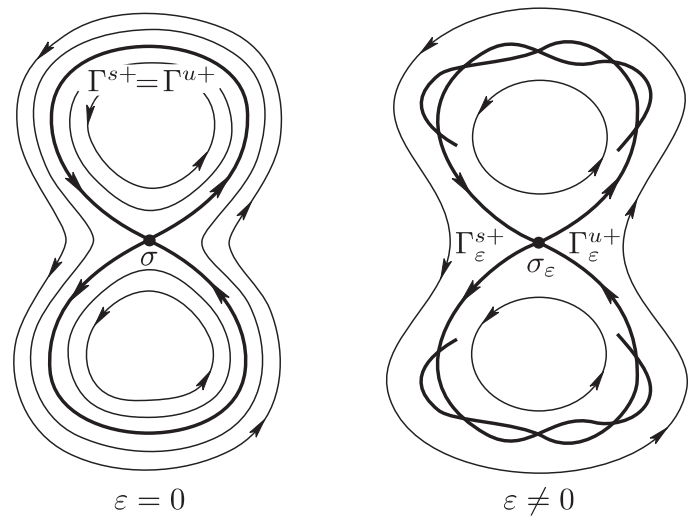

Рис. 3.1. Фазовая плоскость невозмущенной и возмущенной системы

Рассмотрим теперь возмущенное отображение $T_{\varepsilon}, \varepsilon \neq 0$. При малых $\varepsilon$ отображение $T_{\varepsilon}$ имеет гиперболическую неподвижную точку $\sigma_{\varepsilon}$, гладко зависящую от параметра $\varepsilon, \sigma_{0}=\sigma$. Пусть $\Gamma_{\varepsilon}^{u \pm}$ и $\Gamma_{\varepsilon}^{s \pm}-$ сепаратрисные ветви $\sigma_{\varepsilon}$. Вообще говоря, они уже не совпадают при $\varepsilon \neq 0$. Для изучения динамики в окрестности сепаратрис удобным средством является сепаратрисное отображение. Ниже следует его определение.

Рассмотрим окрестность $U_{c}$ сепаратрис: область $|F|<c, c$ мало. Пусть $\Lambda^{+}$ и $\Lambda^{-}$- кривые, идущие от одной компоненты границы к другой трансверсально верхней и нижней сепаратрисной петле соответственно (например, фазовые линии градиентного потока функции $F$ ). Обозначим через $\Delta_{\varepsilon}^{ \pm}$подобласть в $U_{c}$, заключенную между кривыми $\Lambda^{ \pm}$и $T_{\varepsilon}\left(\Lambda^{ \pm}\right)$(см. рис. 3.2). Положим $\Delta_{\varepsilon}=\Delta_{\varepsilon}^{+} \cup \Delta_{\varepsilon}^{-}$. Для достаточно малого $c$ имеем $T_{\varepsilon}\left(\Delta_{\varepsilon}\right) \cap \Delta_{\varepsilon}=\varnothing$. Пусть $z \in \Delta_{\varepsilon}$, и пусть ${ }^{3}$

$$
\begin{gathered}
n_{r}(z)=\min \left\{n \in \mathbb{N}: T_{\varepsilon}(z), \ldots, T_{\varepsilon}^{n-1}(z) \in U_{c}, T_{\varepsilon}^{n}(z) \in \Delta_{\varepsilon}\right\}, \\
\Delta_{\varepsilon}^{r}=\left\{z \in \Delta_{\varepsilon}: n_{r}(z)-\text { определено и конечно }\right\} .
\end{gathered}
$$

Сепаратрисное отображение $\mathscr{S}_{\varepsilon}$ определяется следующим образом:

$$
\mathscr{S}_{\mathscr{M}_{\varepsilon}}: \Delta_{\varepsilon}^{r} \rightarrow \Delta_{\varepsilon}, \quad \mathscr{S}_{\mathscr{M}_{\varepsilon}}(z)=T_{\varepsilon}^{n_{r}}(z)
$$

Обозначим $\bar{\Delta}_{\varepsilon}^{ \pm}=\Delta^{ \pm} \cup \Lambda^{ \pm} \cup T_{\varepsilon}\left(\Lambda^{ \pm}\right)$. Заметим, что $\mathscr{S} \mathscr{M}_{\varepsilon}$ по непрерывности продолжается на $\bar{\Delta}_{\varepsilon}^{ \pm}$так, что $\mathscr{S}_{\varepsilon}(z)=\mathscr{S}_{\mathscr{M}}\left(T_{\varepsilon}(z)\right), z \in \Lambda^{ \pm}$. Поэтому естественно считать, что сепаратрисное отображение определено на подмножестве цилиндров $\bar{\Delta}_{\varepsilon}^{ \pm} / \sim$, где факторизация $\sim$ обозначает отождествление точек $z \in \Lambda^{ \pm}$ и $T_{\varepsilon}(z) \in T_{\varepsilon}\left(\Lambda^{ \pm}\right)$.

Рассмотрение сепаратрисного отображения $\mathscr{S}_{\varepsilon}$ вместо $T_{\varepsilon}$ удобно для исследования динамики в окрестности сепаратрис, потому что автоматически

\footnotetext{
${ }^{3}$ Индекс $r$ в $n_{r}$ и $\Delta_{\varepsilon}^{r}$ взят от слова return (возвращение).
} 

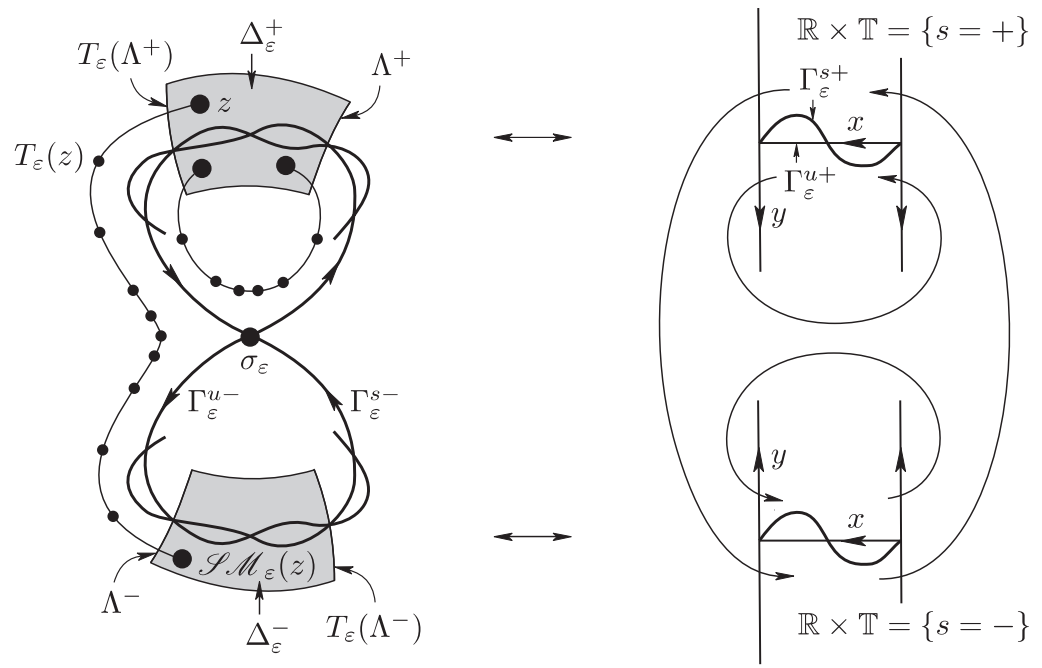

Рис. 3.2. Определение сепаратрисного отображения. Левая часть рисунка представлена в координатах $\left(z_{1}, z_{2}\right)$, а правая в координатах $(x, y, s)$

пропускаются "неинтересные участки" траекторий, проходящие рядом с точкой $\sigma$. Важным обстоятельством является тот факт, что при малых значениях $\varepsilon$ в удобных координатах $\mathscr{S} \mathscr{M} \varepsilon$ допускают простые формулы. А именно, верна следующая теорема.

ТЕОрема 3.1. Пусть выполнены условия I-IV. Тогдана $\bar{\Delta}_{\varepsilon}^{ \pm} / \sim$ существуют гладко зависящие от $\varepsilon$ координаты $x \in \mathbb{T}=\mathbb{R} / \mathbb{Z}, y \in \mathbb{R}, s \in\{ \pm\}$ такие, что

1) $\bar{\Delta}_{\varepsilon}^{+} / \sim=\{s=+\}, \bar{\Delta}_{\varepsilon}^{-} / \sim=\{s=-\}, x$ - угловая координата на иилиндрах $\bar{\Delta}_{\varepsilon}^{ \pm} / \sim$, а геометрический смысл переменной $\varepsilon y-$ расстояние (со знаком) до неустойчивой сепаратрисы;

2) симплектическая структура $d z_{2} \wedge d z_{1}=\varepsilon d y \wedge d x$

3) существуют гладкие функции $\nu_{ \pm}(x)$ и константы $\alpha_{ \pm}$такие, что сепаратрисное отображение имеет вид $\left(x^{+}, y^{+}, s^{+}\right)=\mathscr{S}_{\mathscr{M}}(x, y, s)$,

$$
\left\{\begin{array}{l}
x^{+}=x+\frac{1+O(\varepsilon)}{\lambda}\left(\ln \frac{\varepsilon}{\alpha_{s}^{2} \lambda}+\ln \left|y^{+}\right|\right), \\
y^{+}=y+\lambda \nu_{s}(x)+O(\varepsilon) \\
s^{+}=s \cdot \operatorname{sign}\left(y^{+}\right)
\end{array}\right.
$$

причем $\Delta_{\varepsilon}^{r}=\left\{(x, y) \in \Delta_{\varepsilon}: y^{+} \neq 0\right\}$

4) $\{y=0, s= \pm\} \subset \Gamma_{\varepsilon}^{u \pm},\left\{y^{+}=0, s= \pm\right\} \subset \Gamma_{\varepsilon}^{s \pm}$.

Отметим некоторые свойства отображения (3.1). На окружностях $y^{+}(x$, $y, \pm)=0$ сепаратрисное отображение не определено (соответственно, обратное к нему не определено на окружностях $y=0$ ). Действительно, например, точки, лежащие на отрезках сепаратрис $\Gamma_{\varepsilon}^{s \pm}$, не возвращаются в $\bar{\Delta}_{\varepsilon}^{ \pm}$, а стремятся 
к $\sigma_{\varepsilon}$ при итерациях отображения $T_{\varepsilon}$. Таким образом, динамика для отображения $\mathscr{S}_{\varepsilon}$ может рассматриваться лишь для точек, на траекториях которых переменная $y$ никогда не обращается в нуль, иными словами, вне сепаратрис. Дополнение к указанному множеству точек (след сепаратрис в $\bar{\Delta}_{\varepsilon}^{ \pm}$) состоит из счетного набора гладких кривых и поэтому имеет меру нуль.

Удивительным свойством сепаратрисного отображения является то, что в главном приближении его зависимость от $\ln \varepsilon$ оказывается периодической. Действительно, пренебрегая малыми слагаемыми $O(\varepsilon)$, получаем отображение, которое не меняется при замене $\varepsilon$ на $\varepsilon \mu^{n}=\varepsilon e^{\lambda n}$ для любого целого $n$.

При больших значениях переменной $y$ сепаратрисное отображение близко к интегрируемому. Действительно, полагая $y=y_{0}(1+v)$, где $\left|y_{0}\right|-$ большой параметр, а переменная $v$ мала, получаем:

$$
\begin{aligned}
& x^{+}=x+\frac{1+O(\varepsilon)}{\lambda}\left(\ln \frac{\varepsilon y_{0}}{\alpha_{s}^{2} \lambda}+\ln \left|1+v^{+}\right|\right), \\
& v^{+}=v+O\left(\left|y_{0}\right|^{-1}+|\varepsilon|\right), \\
& s^{+}=s \cdot \operatorname{sign}\left(y_{0}\right) .
\end{aligned}
$$

При $\varepsilon=y_{0}^{-1}=0$ это отображение имеет первый интеграл $v$.

С помощью сепаратрисного отображения легко получить утверждения о неинтегрируемости возмущенной системы. Действительно, предположим, что отображение $T_{\varepsilon}$ аналитично и обладает вещественно-аналитическим первым интегралом. Несложно показать (см., например, [44]), что любой аналитический первый интеграл в нормальных координатах является функцией от $y$. Следовательно, любое интегрируемое отображение $\mathscr{S}_{\varepsilon}$ должно сохранять функцию $y$. Таким образом, согласно (3.1) необходимыми условиями интегрируемости возмущенного отображения $T_{\varepsilon}$ являются равенства $\nu_{ \pm} \equiv 0$ (cp. [45]).

1.2. Гамильтонова система с полутора степенями свободы. Рассмотрим гамильтонову систему с полутора степенями свободы в окрестности асимптотических поверхностей гиперболического периодического решения. В этом случае сепаратрисное отображение естественно определяется путем перехода к отображению за период, но может быть определено и непосредственно как отображение Пуанкаре в расширенном фазовом пространстве. А именно, рассмотрим гамильтонову систему

$$
\dot{z}_{1}=\partial H / \partial z_{2}, \quad \dot{z}_{2}=-\partial H / \partial z_{1}, \quad H(z, t, \varepsilon)=H_{0}(z)+\varepsilon H_{1}(z, t, \varepsilon) .
$$

Здесь $z \in \mathbb{R}^{2}, t \in \mathbb{T}_{t}=\mathbb{R} / \mathbb{Z}$ - время. При $\varepsilon=0$ имеем систему с одной степенью свободы и гамильтонианом $H_{0}$, которую будем называть невозмущенной. Предположим, что

1) невозмущенная система имеет гиперболическое положение равновесия $\sigma,\left.d H_{0}\right|_{z=\sigma}=0$

2) сепаратрисы, выходящие из точки $\sigma$, образуют фигуру типа восъмерки, cм. puc. 3.1 .

Пусть $\lambda>0$ - показатель Ляпунова гиперболического периодического решения $(z(t), t)=(\sigma, t)$ и $\Gamma_{ \pm}(t)$ - сепаратрисные решения невозмущенной системы. Одну из петель восьмерки будем условно называть верхней, а другую - 
нижней. Пусть $\Lambda^{+}, \Lambda^{-} \subset \mathbb{R}_{z}^{2}$ - кривые, трансверсальные верхней и нижней сепаратрисной петле соответственно. Пусть $g_{\varepsilon}^{t}: \mathbb{R}_{z}^{2} \times \mathbb{T}_{t} \hookleftarrow-$ фазовый поток системы (3.2). Сепаратрисное отображение есть отображение Пуанкаре поверхности $\Delta=\left(\Lambda^{+} \times \mathbb{T}_{t}\right) \cup\left(\Lambda^{-} \times \mathbb{T}_{t}\right)$ в расширенном фазовом пространстве $D \times \mathbb{T}_{t}$. Точнее, для любой точки $(z, t) \in \Delta$ положим

$$
t_{r}(z)=\min \left\{t>0: g_{\varepsilon}^{t}(z) \in \Delta\right\}, \quad \Delta_{\varepsilon}^{r}=\left\{(z, t) \in \Delta: t_{r}(z)<+\infty\right\} .
$$

Тогда по определению

$$
\mathscr{S} \mathscr{M}_{\varepsilon}: \Delta_{\varepsilon}^{r} \rightarrow \Delta, \quad \mathscr{S}_{\mathscr{M}}(z)=g_{\varepsilon}^{t_{r}}(z) .
$$

Без ограничения общности можно считать, что сепаратрисная петля - нулевой уровень гамильтониана $H_{0}$.

ТЕОРема 3.2. На поверхности $\Delta$ существуют координаты $(h, t, s) \in \mathbb{R} \times$ $\mathbb{T} \times \mathbb{Z}_{2}$ maкuе, ито

1) $\Lambda^{+} \times \mathbb{T}_{t}=\{s=+\}, \Lambda^{-} \times \mathbb{T}_{t}=\{s=-\}$;

2) $H_{0}(z)=\varepsilon h+O_{2}(\varepsilon)$;

3) $t$ - время;

4) сепаратрисное отображение имеет вид $\left(h^{+}, t^{+}, s^{+}\right)=\mathscr{S}_{\varepsilon}(h, t, s)$,

$$
\left\{\begin{array}{l}
h^{+}=h+I_{s}(t)+O(\varepsilon), \\
t^{+}=t+\frac{1+O(\varepsilon)}{\lambda}\left(\ln \frac{\varepsilon}{\alpha_{s}^{2} \lambda}+\ln \left|h^{+}\right|\right), \\
s^{+}=s \cdot \operatorname{sign}\left(h^{+}\right),
\end{array}\right.
$$

где $\alpha_{ \pm}>0$ - константы, функиии $I_{+}$и $I_{-}$периодические и выражаются через интеграл Пуанкаре-Мельникова:

$$
I_{s}(t)=-\int_{-\infty}^{+\infty}\left\{H_{0}, H_{1}\right\}\left(\Gamma_{s}\left(t-\frac{\ln \alpha_{ \pm}}{\lambda}+\tau\right), \tau\right) d \tau .
$$

ЗАмечАниЕ 3.3. Теоремы 3.1 и 3.2 фактически равносильны друг другу. В самом деле, теорема 3.2 может быть получена, если применить теорему 3.1 к отображению за период $\left.g_{\varepsilon}^{t}\right|_{t=1}$. Это сделано в $\S 6$ этой главы. Благодаря возможности вложения изотопного тождественному $2 n$-мерного симплектического отображения в поток гамильтоновой системы с $n+\frac{1}{2}$ степенями свободы (см. [46], где рассмотрен вещественно-аналитический случай; гладкий случай существенно проще) можно, наоборот, свести теорему 3.1 к теореме 3.2.

\section{§ 2. Отображение склейки: определение}

С этого пункта мы начинаем доказательство теоремы 3.1. Главным инструментом является отображение склейки. Ниже следует его определение.

Пусть $(q, p)=\left(q_{\varepsilon}, p_{\varepsilon}\right)$ - нормальные координаты отображения $T_{\varepsilon}$, определенные в теореме 2.1. В них отображение имеет вид

$$
\mathscr{T}_{\varepsilon}(q, p)=\left(q \mathscr{M}_{\varepsilon}(p q), p / \mathscr{M}_{\varepsilon}(p q)\right),
$$

где, как уже отмечалось ранее, $\sigma_{\varepsilon}=(0,0)$ и $\mu_{\varepsilon}=\mathscr{M}_{\varepsilon}(0)>1$. 
ЗАмечание 3.4. Далее мы считаем, что нормальные координаты выбраны таким образом, что внутри верхней петли “восьмерки" $q>0$ и $p>0$, см. рис. 3.3.

Нормальные координаты, первоначально определенные лишь в малой окрестности гиперболической точки, можно продолжить вдоль сепаратрис с помощью следующей индуктивной процедуры. Пусть координаты точки $z \in D$ известны и равны $(q, p)$. Тогда координаты точек $T(z)$ и $T^{-1}(z)$ определим как $\mathscr{T}(q, p)$ и $\mathscr{T}^{-1}(q, p)$ соответственно. Вдали от гиперболической точки $\sigma_{\varepsilon}$ получаем, по крайней мере, два различных продолжения нормальных координат вдоль устойчивой и неустойчивой сепаратрисы. Возникающее отождествление координат называется отображением склейки. Более формально эта конструкция выглядит следующим образом.
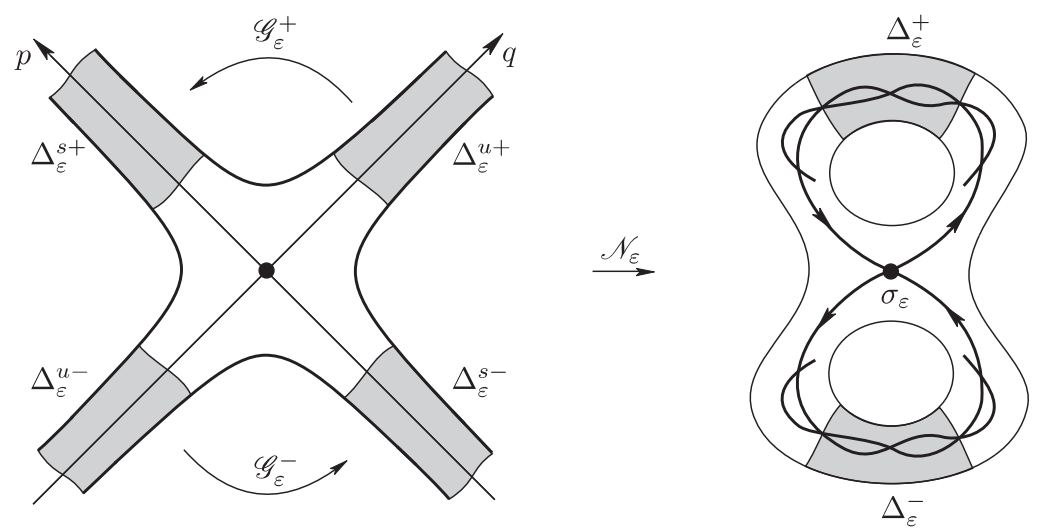

Рис. 3.3. Отображение склейки

Пусть $\mathscr{U}_{\varepsilon}$ - окрестность, в которой определены нормальные координаты $(q, p)$. Обозначим через $\mathscr{N}_{\varepsilon}: U_{\varepsilon} \rightarrow \mathscr{U}_{\varepsilon}$ отображение, задающее нормальные координаты, $U_{\varepsilon} \subset \mathbb{R}_{q, p}^{2}$. Зафиксируем положительные целые числа $n_{u}$ и $n_{s}$. Пусть

$$
U_{\varepsilon}^{n_{s}, n_{u}}=\bigcup_{-n_{s} \leqslant n \leqslant n_{u}} \mathscr{T}_{\varepsilon}^{n}(U), \quad \mathscr{U}_{\varepsilon}^{n_{s}, n_{u}}=\bigcup_{-n_{s} \leqslant n \leqslant n_{u}} T_{\varepsilon}^{n}(\mathscr{U}) .
$$

Продолжим отображение $\mathscr{N}$ на $U_{\varepsilon}^{n_{s}, n_{u}}$ так, что следующая диаграмма коммутативна:

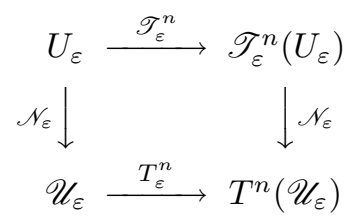

Это продолжение корректно определено, поскольку $\mathscr{N}_{\varepsilon} \circ \mathscr{T}_{\varepsilon}=T_{\varepsilon} \circ \mathscr{N}_{\varepsilon}$ в $U_{\varepsilon}$.

При больших $n_{s}$ и $n_{u}$ отображение $\mathscr{N}_{\varepsilon}$ перестает быть инъективным. Выберем $n_{s}$ и $n_{u}$ так, чтобы отображение $\mathscr{N}_{\varepsilon}$ задавало двулистное накрытие фундаментальных областей $\Delta_{\varepsilon}^{ \pm}$. 
Обозначим через $\Delta_{\varepsilon}^{u \pm}$ и $\Delta_{\varepsilon}^{s \pm}$ прообразы областей $\Delta_{\varepsilon}^{ \pm}$, лежащие в окрестности неустойчивых и устойчивых сепаратрис соответственно:

$$
\begin{aligned}
& \Delta_{\varepsilon}^{u \pm} \subset\left\{(q, p): c< \pm q<c^{-1}, p \text { мало }\right\} \\
& \Delta_{\varepsilon}^{s \pm} \subset\left\{(q, p): q \text { мало, } c< \pm p<c^{-1}\right\} .
\end{aligned}
$$

Тогда определение отображения склейки имеет следующий вид:

$$
\mathscr{G}_{\varepsilon}^{ \pm}: \Delta_{\varepsilon}^{u \pm} \rightarrow \Delta_{\varepsilon}^{s \pm}, \quad \mathscr{G}_{\varepsilon}^{ \pm}=\left.\left(\left.\mathscr{N}_{\varepsilon}\right|_{\Delta_{\varepsilon}^{s \pm}}\right)^{-1} \circ \mathscr{N}_{\varepsilon}\right|_{\Delta_{\varepsilon}^{u \pm}}
$$

Если обозначить $\left(q_{u}, p_{u}\right)$ и $\left(q_{s}, p_{s}\right)$ нормальные координаты точки $z \in \Delta_{\varepsilon}^{ \pm}$, полученные продолжением вдоль неустойчивой и устойчивой сепаратрисы соответственно: $\mathscr{N}_{\varepsilon}\left(q_{u}, p_{u}\right)=z,\left(q_{u}, p_{u}\right) \in \Delta_{\varepsilon}^{u \pm}$ и $\mathscr{N}_{\varepsilon}\left(q_{s}, p_{s}\right)=z,\left(q_{s}, p_{s}\right) \in \Delta_{\varepsilon}^{s \pm}$, то они отождествляются отображением склейки $\mathscr{G}_{\varepsilon}^{ \pm}\left(q_{u}, p_{u}\right)=\left(q_{s}, p_{s}\right)$.

Имея явные формулы для $\mathscr{G}_{\varepsilon}^{ \pm}$, динамику отображения $T_{\varepsilon}$ в окрестности сепаратрис можно изучать в нормальных координатах. Действительно, нормальные координаты осуществляют гладкое отображение крестообразной области, изображенной слева на рис. 3.3, на окрестность сепаратрис симплектоморфизма $T_{\varepsilon}$. Пусть начальные условия заданы в нормальных координатах. Для построения траектории системы можно сначала применить несколько раз гиперболический поворот $\mathscr{T}_{\varepsilon}$. При этом итерации точки начнут удаляться вдоль "северо-восточного" или "юго-западного" рукава креста и в конце концов попадут в область определения одного из отображений склейки. После применения отображения $\mathscr{G}_{\varepsilon}^{+}$или $\mathscr{G}_{\varepsilon}^{-}$опять можно применять отображение $\mathscr{T}_{\varepsilon}$ и т.д. При этом ясно, что почти вся информация о динамике содержится в отображениях склейки, так как формулы для гиперболического поворота для различных систем имеют несущественные различия.

\section{§ 3. Отображение склейки: формулы}

Лемма 3.5. Пусть $C^{4}$-гладкая функиця $F(q, p)$ - первый интеграл гиперболического поворота $\mathscr{T}(q, p)=(q \mathscr{M}(p q), p / \mathscr{M}(p q))$. Тогда

$$
F(q, p)=F_{0}+F_{1} q p+O_{2}(q p), \quad F_{0}, F_{1}=\text { const. }
$$

ЗАмечАниЕ 3.6. Если $F$ - аналитическая функция переменных $(q, p)$, то, более того, $F(q, p)=\mathscr{F}(q p)[42]$.

ДокАЗАТЕЛЬСтво ЛЕммы 3.5. Очевидно, что $F=F_{0}=$ const на сепаратри$\operatorname{cax} q=0$ и $p=0$. Поэтому $F=F_{0}+O(q p)=F_{0}+q p G$. Поскольку $F$ и $q p-$ интегралы отображения $\mathscr{T}$, то $G$ тоже первый интеграл. Поэтому $G=F_{1}+O(q p)$. Отсюда следует (3.7).

Отображения склейки, соответствующие интегрируемым системам, выглядят довольно просто. А именно, справедливо следующее предложение. 
ПРЕДЛОЖЕНИЕ 3.7. Пусть выполнены условия теоремы 3.1. Тогда отображения склейки для отображения $T_{0}$ имеют вид $\left(q_{s}, p_{s}\right)=\mathscr{G}^{ \pm}\left(q_{u}, p_{u}\right)$,

$$
\left\{\begin{array}{l}
q_{s}=\frac{q_{u}^{2} p_{u}}{\alpha_{ \pm}^{2}}+O_{2}\left(q_{u} p_{u}\right), \\
p_{s}=\frac{\alpha_{ \pm}^{2}}{q_{u}}+O\left(q_{u} p_{u}\right)
\end{array}\right.
$$

где $\alpha_{ \pm}-$положительные постоянные.

ДоказАтельство. Пусть $\mathscr{G}^{+}\left(q_{u}, p_{u}\right)=\left(q_{s}, p_{s}\right)$. Тогда

$$
F\left(q_{s}, p_{s}\right)=F\left(q_{u}, p_{u}\right), \quad d p_{s} \wedge d q_{s}=d p_{u} \wedge d q_{u} .
$$

Из условия IV теоремы 3.1 следует, что $F_{1} \neq 0$. Тогда

$$
q_{s} p_{s}=q_{u} p_{u}+\mathrm{O}_{2}\left(q_{u} p_{u}\right)
$$

Подставляя равенство $q_{s}=\frac{1}{p_{s}}\left(q_{u} p_{u}+O_{2}\left(q_{u} p_{u}\right)\right)$ в $(3.9)$, получаем следующее уравнение в частных производных:

$$
p_{u} \frac{\partial p_{s}}{\partial p_{u}}-q_{u} \frac{\partial p_{s}}{\partial q_{u}}=p_{s}\left(1+O\left(q_{u} p_{u}\right)\right) .
$$

Решения $p_{s}=p_{s}\left(q_{u}, p_{u}\right)$, гладкие при $p_{u}=0$, имеют вид $p_{s}=q_{u}^{-1}\left(a_{0}+O\left(q_{u} p_{u}\right)\right)$. Неравенство $a_{0}>0$ следует из принятого в замечании 3.4 соглашения о выборе нормальных координат, откуда получаем второе равенство (3.8). Первое равенство (3.8) теперь следует из (3.10). Предложение 3.7 доказано.

Отображения склейки для возмущенного отображения $T_{\varepsilon}$ получается возмущением отображения (3.8).

ПРЕДЛОЖЕНИЕ 3.8. Пусть выполнены условия теоремы 3.1. Тогда отображения склейки отображения $T_{\varepsilon}$ имеют вид $\left(q_{s}, p_{s}\right)=\mathscr{G}_{\varepsilon}^{ \pm}\left(q_{u}, p_{u}\right)$,

$$
\left\{\begin{array}{l}
q_{s}=q_{u} \frac{q_{u} p_{u}+\varepsilon \nu_{ \pm}\left(\ln \left|q_{u}\right| / \ln \mu\right)}{\alpha_{ \pm}^{2}}+O_{2}\left(q_{u} p_{u}, \varepsilon\right), \\
p_{s}=\frac{\alpha_{ \pm}^{2}}{q_{u}}+O\left(q_{u} p_{u}, \varepsilon\right),
\end{array}\right.
$$

где $\nu_{ \pm}-$периодические функиии с периодом 1.

ДоказАтельство. Действительно, имеем

$$
\mathscr{G}_{\varepsilon}^{ \pm}\left(q_{u}, p_{u}\right)=\mathscr{G}_{0}^{ \pm}\left(q_{u}, p_{u}\right)+\varepsilon\left(f_{ \pm}\left(q_{u}, p_{u}, \varepsilon\right), g_{ \pm}\left(q_{u}, p_{u}, \varepsilon\right)\right)
$$

где $f_{ \pm}, g_{ \pm}$- гладкие функции. Согласно (3.6) имеем $f_{ \pm}\left(\mu q_{u}, 0,0\right)=\mu f_{ \pm}\left(q_{u}, 0,0\right)$, где $\mu=\mathscr{M}_{0}(0)$. Положим

$$
f_{ \pm}\left(q_{u}, 0,0\right)=q_{u} \frac{1}{\alpha_{ \pm}^{2}} \nu_{ \pm}\left(\ln \left|q_{u}\right| / \ln \mu\right) .
$$


Функции $\nu_{ \pm}$гладкие и периодические с периодом 1 . Тогда

$$
\begin{aligned}
\mathscr{G}_{\varepsilon}^{ \pm}\left(q_{u}, p_{u}\right) & =\mathscr{G}_{0}^{ \pm}\left(q_{u}, p_{u}\right)+\varepsilon\left(q_{u} \frac{\nu_{ \pm}\left(\ln \left|q_{u}\right| / \ln \mu\right)}{\alpha_{ \pm}^{2}}+O\left(p_{u}\right), g_{ \pm}\left(q_{u}, p_{u}, \varepsilon\right)\right) \\
& =\left(q_{u} \frac{q_{u} p_{u}+\varepsilon \nu_{ \pm}\left(\ln \left|q_{u}\right| / \ln \mu\right)}{\alpha_{ \pm}^{2}}, \frac{\alpha_{ \pm}^{2}}{q_{u}}\right)+\left(O_{2}\left(q_{u} p_{u}, \varepsilon\right), O\left(q_{u} p_{u}, \varepsilon\right)\right) .
\end{aligned}
$$

Предложение 3.8 доказано.

\section{§ 4. Сепаратрисное отображение}

ДокАЗАТЕЛЬСТво тЕОРЕмы 3.1. На областях $\Delta_{\varepsilon}^{ \pm}$имеются две системы координат $\left(q_{s}, p_{s}\right)$ и $\left(q_{u}, p_{u}\right)$, заданные отображениями $\left.\mathscr{N}_{\varepsilon}\right|_{\Delta_{\varepsilon}^{s \pm}}$ и $\left.\mathscr{N}_{\varepsilon}\right|_{\Delta_{\varepsilon}^{u \pm}}$ соответственно. В последней системе координат сепаратрисное отображение имеет вид

$$
\mathscr{S}_{\mathscr{M}}=\mathscr{T}_{\varepsilon}^{n_{r}} \circ \mathscr{G}_{\varepsilon}
$$

где, напомним, $n_{r}(z)$ - количество итераций точки $z \in \Delta_{\varepsilon}^{r}$ при отображении $T_{\varepsilon}$ до первого возвращения в $\Delta_{\varepsilon}$. В нормальных координатах $n_{r}(q, p)$, где $(q, p) \in \Delta_{\varepsilon}^{s \pm}$, это количество итераций отображения $\mathscr{T}_{\varepsilon}$ до попадания точки в области $\Delta_{\varepsilon}^{u \pm}$.

Вне устойчивой сепаратрисы $\{q=0\}$ определены следующие переменные:

$$
\begin{aligned}
& x=\frac{\ln |q|}{\ln \mathscr{M}_{\varepsilon}(q p)}=\frac{\ln |q|}{\lambda}(1+O(q p, \varepsilon)), \\
& y=\varepsilon^{-1} \int_{0}^{q p} \ln \mathscr{M}_{\varepsilon}(\xi) d \xi=\varepsilon^{-1} q p(\lambda+O(q p, \varepsilon)) .
\end{aligned}
$$

Легко проверить, что $d y \wedge d x=\varepsilon^{-1} d p \wedge d q$. В переменных $(x, y)$ гиперболический поворот $\mathscr{T}_{\varepsilon}$ имеет вид

$$
\mathscr{T}_{\varepsilon}(x, y)=(x+1, y) .
$$

Поэтому отображение $\left.\mathscr{N}_{\varepsilon}\right|_{\Delta_{\varepsilon}^{u \pm}}(x, y)$ задает гладкие координаты $(x \bmod 1, y)$ на цилиндрах $\bar{\Delta}_{\varepsilon}^{ \pm} / \sim$. Чтобы восстановить информацию о знаке переменной $q$ (утерянную в $(3.13))$, добавим знак $s=\operatorname{sign}(q)$ к системе координат $(x, y)$. При этом если $s=+$ (соответственно $s=-$ ), то $(x, y) \in \Delta_{\varepsilon}^{u+}$ (соответственно $\left.(x, y) \in \Delta_{\varepsilon}^{u-}\right)$. Сепаратрисное отображение записывается в координатах $(x \bmod 1, y, s)$.

Считая $x$ определенным по модулю 1 , из (3.12) и (3.14) получаем, что $\mathscr{S M}_{\varepsilon}$ определяется только отображением склейки $\mathscr{G}_{\varepsilon}$. Остается записать отображение склейки в переменных $(x, y)$.

Обращая равенства (3.13), имеем:

$$
q p=\varepsilon y\left(\frac{1}{\lambda}+O(\varepsilon)\right), \quad q=s e^{\lambda x(1+O(\varepsilon))} .
$$


Пусть $\left(x_{u}, y_{u}\right) \in \bar{\Delta}_{\varepsilon}^{u \pm}$ и $\left(x_{s}, y_{s}\right)=\mathscr{G}_{\varepsilon}^{ \pm}\left(x_{u}, y_{u}\right)$. Из предложения (3.11) последовательно получаем:

$$
\begin{aligned}
q_{s} p_{s} & =q_{u} p_{u}+\varepsilon \nu_{ \pm}\left(\ln \left|q_{u}\right| / \ln \mu\right)+O_{2}\left(q_{u} p_{u}, \varepsilon\right)=\frac{1}{\lambda} \varepsilon y_{u}+\varepsilon \nu_{ \pm}\left(x_{u}\right)+O_{2}(\varepsilon), \\
y_{s} & =\varepsilon^{-1} q_{s} p_{s}\left(\lambda+O\left(q_{s} p_{s}, \varepsilon\right)\right)=y_{u}+\lambda \nu_{ \pm}\left(x_{u}\right)+O(\varepsilon), \\
x_{s} & =\frac{\ln \left|q_{s} p_{s} / p_{s}\right|}{\lambda}\left(1+O\left(q_{s} p_{s}, \varepsilon\right)\right)=\frac{1+O(\varepsilon)}{\lambda}\left(\ln \left|q_{u}\right|+\ln \frac{\varepsilon}{\alpha_{ \pm}^{2} \lambda}+\ln (1+O(\varepsilon))\right) \\
& =x_{u}+\frac{1+O(\varepsilon)}{\lambda}\left(\ln \frac{\varepsilon}{\alpha_{ \pm}^{2} \lambda}+\ln \left|y_{s}\right|+O(\varepsilon)\right) .
\end{aligned}
$$

Также заметим, что $\operatorname{sign}\left(q_{s}\right)=\operatorname{sign}\left(y_{s}\right) \cdot \operatorname{sign}\left(q_{u}\right)$. Окончательно, обозначая $\mathscr{S} \mathscr{M}_{\varepsilon}(x, y, s)=\left(x^{+}, y^{+}, s^{+}\right)$, получаем

$$
\left\{\begin{array}{l}
x^{+}=x+\frac{1+O(\varepsilon)}{\lambda}\left(\ln \frac{\varepsilon}{\alpha_{s}^{2} \lambda}+\ln \left|y^{+}\right|\right), \\
y^{+}=y+\lambda \nu_{s}(x)+O(\varepsilon) \\
s^{+}=s \cdot \operatorname{sign}\left(y^{+}\right) .
\end{array}\right.
$$

Теорема 3.1 доказана.

\section{§ 5. Интеграл Пуанкаре-Мельникова}

В этом параграфе мы покажем, как найти в явном виде сепаратрисное отображение в главном приближении. Точнее, мы получим формулы для параметров $\alpha_{ \pm}$и функций $\nu_{ \pm}$. Доказательства имеются в [47], см. также [44].

Пусть $F$ - первый интеграл отображения $T_{0}, F(\sigma)=0$ и критическая точка $\sigma$ функции $F$ невырождена. Пусть $\Gamma^{ \pm}: \mathbb{R} \rightarrow\{z \in D: F(z)=0\}-$ верхняя и нижняя петли невозмущенной сепаратрисы, параметризованные естественным образом:

$$
T_{0} \circ \Gamma^{ \pm}(t)=\Gamma^{ \pm}(t+1) .
$$

В примерах часто функции $\Gamma^{ \pm}$удается выписать в явном виде.

В окрестности точки $\sigma$ можно ввести симплектические координаты $(u, v)$ такие, что кривые $\Gamma^{ \pm}(t)$ удовлетворяют соотношениям

$$
\begin{aligned}
& (u, v)=\left(c_{u}^{ \pm} \mu^{t}+O\left(\mu^{2 t}\right), O\left(\mu^{2 t}\right)\right) \quad \text { при } t \rightarrow-\infty, \\
& (u, v)=\left(O\left(\mu^{-2 t}\right), c_{s}^{ \pm} \mu^{-t}+O\left(\mu^{-2 t}\right)\right) \quad \text { при } t \rightarrow+\infty \text {, }
\end{aligned}
$$

где $c_{s, u}^{+}>0$ и $c_{s, u}^{-}<0$. Эти координаты можно получить линейной заменой из любых симплектических координат, определенных в окрестности неподвижной точки $\sigma$. Постоянные $c_{s, u}^{ \pm}$определены неоднозначно, но только одна из них может быть выбрана произвольным образом.

ПрЕДЛОЖЕНИЕ 3.9. (а) Величины $c_{s}^{+} c_{u}^{+}, c_{s}^{-} c_{u}^{-}, c_{s}^{+} / c_{s}^{-}$не зависят от выбора переменных $(u, v)$.

(б) Величинь $c_{s}^{+} c_{u}^{+}, c_{s}^{-} c_{u}^{-}$сохраняются при заменах параметризаций $t \mapsto$ $t+\widehat{c}^{ \pm}$на кривъх $\Gamma^{ \pm}$.

(в) $\alpha_{ \pm}^{2}=c_{s}^{ \pm} c_{u}^{ \pm}$. 
СлЕДСТВИЕ 3.10. Величины $\alpha_{ \pm}$вычисляются, если известна естественная параметризачия невозмущенной сепаратрисъ.

Функции $\nu_{ \pm}$можно получить из дискретного аналога интеграла ПуанкареМельникова. Положим

$$
w\left(T_{0}(z)\right)=\left.\frac{d}{d \varepsilon}\right|_{\varepsilon=0} T_{\varepsilon}(z), \quad \nu_{ \pm}^{*}(t)=\sum_{n=-\infty}^{\infty} d F \cdot w\left(\Gamma^{ \pm}(t+n)\right),
$$

где выражение $d F \cdot w(A)$ обозначает применение ковектора $d F$ к вектору $w$ в точке $A \in D$.

Так как критическая точка $\sigma$ невырождена, выполняется равенство

$$
F(u, v)=F_{0} u v+O_{3}(u, v), \quad F_{0} \neq 0 .
$$

ПРеДЛОЖениЕ 3.11. Справедливы следующие соотношения:

$$
\nu_{ \pm}(t)=F_{0}^{-1} \nu_{ \pm}^{*}\left(t-\ln \alpha_{ \pm} / \ln \mu\right) .
$$

\section{§ 6. Гамильтонова система}

\section{Здесь мы выведем теорему 3.2 из теоремы 3.1.}

Пусть симплектоморфизм $T_{\varepsilon}$ есть отображение за период гамильтоновой системы с гамильтонианом $H(z, t, \varepsilon)$. Нормальные координаты $(q, p)=\left(q_{\varepsilon}, p_{\varepsilon}\right)$ отображения $T_{\varepsilon}$ из теоремы 2.1 порождают нормальные координаты для гамильтоновой системы (см. теорему 2.3). Действительно, достаточно положить нормальные координаты точки $g_{\varepsilon}^{t}(z)$ равными $\left(q \mathscr{M}_{\varepsilon}^{t}, p \mathscr{M}_{\varepsilon}^{-t}\right)$, где $(q, p)-$ нормальные координаты точки $z, g_{\varepsilon}^{t}$ - фазовый поток гамильтоновой системы. В нормальных координатах фазовый поток имеет вид

$$
g_{\varepsilon}^{t}(q, p)=\left(q e^{\mathscr{H}_{\varepsilon}^{\prime}(p q) t}, p e^{-\mathscr{H}_{\varepsilon}^{\prime}(p q) t}\right) .
$$

Сравнивая $\left.g_{\varepsilon}^{t}\right|_{t=1}$ и $(2.1)$, получим, что функции $\mathscr{M}_{\varepsilon}(p q)$ и $\mathscr{H}_{\varepsilon}(p q)$ из теорем 2.1 и 2.3 связаны равенством

$$
\frac{d \mathscr{H}_{\varepsilon}(\xi)}{d \xi}=\ln \mathscr{M}_{\varepsilon}(\xi) .
$$

Поскольку невозмущенный гамильтониан $H_{0}$ не зависит от времени, то зависимость от времени в нормализующей замене координат присутствует только в членах порядка $\varepsilon$. Поэтому новый гамильтониан $\mathscr{H}_{\varepsilon}=H_{\varepsilon}+O(\varepsilon)$.

Вне устойчивой сепаратрисы $\{q=0\}$ на $\mathbb{R}_{q, p}^{2}$ введем координаты “энергиявремя" $(h, t)$. Положим $t=0$ при $q= \pm 1$. Считая $\mathscr{H}_{\varepsilon}(0)=0$, из $(3.20)$ и $(3.19)$ имеем:

$$
t=\frac{\ln \left|q_{\varepsilon}\right|}{\ln \mathscr{M}_{\varepsilon}}, \quad h=\varepsilon^{-1} \mathscr{H}_{\varepsilon}=\varepsilon^{-1} \int_{0}^{q_{\varepsilon} p_{\varepsilon}} \ln \mathscr{M}_{\varepsilon}(\xi) d \xi .
$$

Таким образом, координаты $(h, t)$ совпадают с координатами $(y, x)($ см. $(3.13))$. Поэтому из (3.15) получаем $\mathscr{S}_{\mathcal{M}}(h, t, s)=\left(h^{+}, t^{+}, s^{+}\right)$:

$$
\left\{\begin{array}{l}
h^{+}=h+\lambda \nu_{s}(t)+O(\varepsilon), \\
t^{+}=t+\frac{1+O(\varepsilon)}{\lambda}\left(\ln \frac{\varepsilon}{\alpha_{s}^{2} \lambda}+\ln \left|h^{+}\right|\right), \\
s^{+}=s \cdot \operatorname{sign}\left(h^{+}\right) .
\end{array}\right.
$$


Из предложения 3.11 получаем следующее

ПРЕДЛОЖЕНИЕ 3.12.

$$
\nu_{ \pm}(t)=\frac{1}{\lambda} \nu_{ \pm}^{*}\left(t-\ln \alpha_{ \pm} / \ln \mu\right),
$$

а функции $\nu_{ \pm}^{*}$ можно представить в виде интеграла Пуанкаре-Мельникова:

$$
-\nu_{ \pm}^{*}(t)=\int_{-\infty}^{+\infty}\left\{H_{0},\left.H_{1}\right|_{\varepsilon=0}\right\}\left(\Gamma^{ \pm}(t+\xi), \xi\right) d \xi .
$$

ДокАЗАтельство. Пусть $z_{\varepsilon}(t)=z_{0}(t)+\varepsilon z^{\prime}(t)+O\left(\varepsilon^{2}\right)-$ решение системы с гамильтонианом $H$ и начальными условиями $z_{\varepsilon}(0)=(u, v)$. Тогда

$$
T_{\varepsilon}(u, v)=z_{\varepsilon}(1)
$$

Ниже будем считать, что точка $(u, v)$ не зависит от $\varepsilon$. Тогда, дифференцируя (3.22) по $\varepsilon$ в точке $\varepsilon=0$, получаем:

$$
w\left(T_{0}(u, v)\right)=z^{\prime}\left(\tau_{0}\right) .
$$

Дифференцируя тождество $\frac{d}{d t} H_{0}\left(z_{\varepsilon}(t)\right)=\left\{H_{1}, H_{0}\right\}\left(z_{\varepsilon}(t), t, \varepsilon\right)$ по $\varepsilon$ и полагая $\varepsilon=0$, получаем следующее равенство:

$$
\frac{d}{d t}\left(d H_{0}\left(z_{0}(t)\right) \cdot z^{\prime}(t)\right)=\varepsilon\left\{H_{1}, H_{0}\right\}\left(z_{0}(t)\right) .
$$

Положим $z_{0}(t)=\Gamma^{+}\left(t_{0}+t\right)$. Интегрируя (3.24), имеем:

$$
\begin{gathered}
d H_{0}\left(\Gamma^{+}\left(t_{0}+1\right)\right) \cdot z^{\prime}(1)-d H_{0}\left(\Gamma^{+}\left(t_{0}\right)\right) \cdot z^{\prime}(0) \\
=\int_{0}^{1}\left\{H_{1}, H_{0}\right\}\left(\Gamma^{+}\left(t_{0}+t\right), t\right) d t .
\end{gathered}
$$

Пользуясь равенством (3.23) и тем, что $z^{\prime}(0)=0$, получаем:

$$
d H_{0} \cdot w\left(\Gamma^{+}\left(t_{0}+1\right)\right)=\int_{0}^{1}\left\{H_{1}, H_{0}\right\}\left(\Gamma^{+}\left(t_{0}+\xi\right), \xi\right) d \xi .
$$

Соответствующее равенство для $\Gamma^{-}$находится аналогично. Из этих равенств и определения функции $\nu_{+}^{*}(3.18)$ следует предложение 3.12.

\section{§ 7. Сепаратрисное отображение для маятника}

Рассмотрим в качестве примера маятник, точка подвеса которого периодически колеблется вдоль вертикали. Гамильтониан системы имеет вид

$$
H(\widehat{u}, \widehat{v}, t, \varepsilon)=\widehat{v}^{2} / 2+\Omega^{2} \cos \widehat{u}+\varepsilon \theta(\omega t) \cos \widehat{u} .
$$

Здесь $\widehat{u}=\widehat{u} \bmod 2 \pi-$ угол между маятником и вертикалью, $\widehat{v}-$ соответствующий импульс, $\Omega>0$ - "внутренняя частота" системы $\left(\Omega^{2}\right.$ равна ускорению силы 
тяжести, деленному на длину маятника), $\omega$ - частота колебаний точки подвеса и параметр $\varepsilon$ пропорционален амплитуде колебаний, умноженной на $\omega^{2}$. Закон колебаний точки подвеса определяется $2 \pi$-периодической функцией $\theta$. Предположим, что параметр $\varepsilon$ мал, а другие параметры в системе - порядка единицы.

Симплектической заменой переменных сделаем систему 1-периодической. Пусть новое время $\tau=\omega t /(2 \pi)$ и новый гамильтониан $\mathscr{H}=2 \pi H / \omega$. Отображение Пуанкаре в этой системе имеет гиперболическую неподвижную точку $\widehat{u}=\widehat{v}=0$. Несложно вычислить мультипликатор: $\mu=e^{2 \pi \Omega / \omega}$. Тогда $\lambda=\ln \mu=2 \pi \Omega / \omega$. Сепаратрисы $\Gamma^{ \pm}$имеют вид:

$$
(\widehat{u}, \widehat{v})=\left(4 \operatorname{arctg} e^{ \pm 2 \pi \Omega \tau / \omega}, \pm 2 \Omega / \operatorname{ch}(2 \pi \Omega \tau / \omega)\right) .
$$

Переменные $(u, v)$ (см. (3.17)) - следующие:

$$
u=\widehat{u} \sqrt{\Omega / 2}+\widehat{v} / \sqrt{2 \Omega}, \quad v=-\widehat{u} \sqrt{\Omega / 2}+\widehat{v} / \sqrt{2 \Omega} .
$$

Получаем: $c_{s}^{+}=c_{u}^{+}=4 \sqrt{2 \Omega}$ и $c_{s}^{-}=c_{u}^{-}=-4 \sqrt{2 \Omega}$. Следовательно, согласно предложению $3.9, \alpha_{ \pm}^{2}=32 \Omega$.

С помощью предложения 3.12 получаем:

$$
\nu_{ \pm}^{*}(\tau)=\left.\left(\frac{2 \pi}{\omega}\right)^{2} \int_{-\infty}^{+\infty} \theta(2 \pi \xi)(\widehat{v} \sin \widehat{u})\right|_{\Gamma^{ \pm}(\tau+\xi)} d \xi
$$

Прямые вычисления приводят к равенству

$$
\nu_{ \pm}^{*}(\tau)=-\frac{4 \pi^{2} i \omega}{\Omega^{2}} \sum_{m \in \mathbb{Z} \backslash\{0\}} \frac{m^{2} \theta_{m}}{\operatorname{sh}(\pi m \omega /(2 \Omega))} e^{-2 \pi i m \tau},
$$

где разложение Фурье функции $\theta$ имеет вид

$$
\theta(s)=\sum_{m \in \mathbb{Z}} \theta_{m} e^{i m s} .
$$

В частности, $\nu_{ \pm}^{*} \not \equiv$ const при $\theta \not \equiv$ const и, следовательно, при $\theta \not \equiv$ const возмущенная система не имеет аналитического первого интеграла для любого малого $\varepsilon \neq 0$.

\section{§ 8. Сепаратрисное отображение в общем случае}

Целью настоящего параграфа является указание единой схемы для построения сепаратрисного отображения в системах, рассмотренных в 11 этой главы и в тех системах $T_{\varepsilon}$, где $T_{0}$ является тождественным отображением и поэтому при $\varepsilon=0$ гиперболическая неподвижная точка отсутствует. Стандартный пример систем второго типа рассмотрен во введении (§1), где речь идет о разрушении резонансной кривой интегрируемого отображения. Другим примером является маятник с быстро колеблющейся точкой подвеса.

В системах второго типа мультипликатор $\mu_{\varepsilon}$ гиперболической неподвижной точки $\sigma_{\varepsilon}$ стремится к нулю при $\varepsilon \searrow 0$, а расщепление сепаратрис экспоненциально мало по $\varepsilon$. Методами теории усреднения (см. [48]) возмущенную систему удается приблизить интегрируемым отображением, которое также имеет 
гиперболическую неподвижную точку. Причем в случае аналитических симплектоморфизмов разность между этими системами можно сделать экспоненциально малой по $\varepsilon$. Далее эта разность характеризуется параметром $\delta=\delta_{\varepsilon}$. Следовательно, если рассматривать возмущенную систему как деформацию интегрируемой по параметру $\delta$, то конструкция сепаратрисного отображения аналогична изложенному выше случаю.

Пусть $T_{\varepsilon}, 0<\varepsilon<\varepsilon_{0},-$ гладкое семейство двумерных симплектоморфизмов, близких к интегрируемому $T_{0}$. Предположим, что

(A) существует гладкое семейство интегрируемых отображений $\widetilde{T}_{\varepsilon}, 0<$ $\varepsilon<\varepsilon_{0}$, для которых выполнены условия теоремы 3.1, и таких, что $\left|T_{\varepsilon}-\widetilde{T}_{\varepsilon}\right| \leqslant$ $\delta_{\varepsilon}$, где $\delta_{\varepsilon} \rightarrow 0$ при $\varepsilon \rightarrow 0$.

Если само отображение $T_{0}$ удовлетворяет условиям теоремы 3.1 , то можно взять $\widetilde{T}_{\varepsilon} \equiv T_{0}$ и $\delta_{\varepsilon}=\varepsilon$.

Поскольку отображения $\widetilde{T}_{\varepsilon}$ удовлетворяют условиям теоремы 3.1 , то отображения склейки для них имеют вид (3.8), где $\alpha_{ \pm}=\alpha_{ \pm}(\varepsilon)$. Тогда отображения склейки для $T_{\varepsilon}$ имеют вид:

$$
\left\{\begin{array}{l}
q_{s}=\frac{q_{u}^{2} p_{u}}{\alpha_{ \pm}^{2}(\varepsilon)}+\delta_{\varepsilon} q_{u} f_{ \pm}\left(q_{u}, p_{u}, \varepsilon\right)+O_{2}\left(q_{u} p_{u}\right) \\
p_{s}=\frac{\alpha_{ \pm}^{2}(\varepsilon)}{q_{u}}+\delta_{\varepsilon} p_{u} g_{ \pm}\left(q_{u}, p_{u}, \varepsilon\right)+O_{1}\left(q_{u} p_{u}\right)
\end{array}\right.
$$

Здесь $(q, p)=(q(\varepsilon), p(\varepsilon))$ - нормальные координаты отображений $T_{\varepsilon}$ в окрестности гиперболической неподвижной точки $\sigma_{\varepsilon},\left(q_{u}, p_{u}\right)$ и $\left(q_{s}, p_{s}\right)$ - продолженные координаты вдоль неустойчивой и устойчивой сепаратрис $\sigma_{\varepsilon}$ соответственно.

Пусть $\mu_{\varepsilon}=\mathscr{M}_{\varepsilon}(0)$ - мультипликатор гиперболической неподвижной точки $\sigma_{\varepsilon}$ отображения $T_{\varepsilon}, \mathscr{M}_{\varepsilon}(q p)$ - функция, задающая $T_{\varepsilon}$ в нормальных координатах (см. теорему 2.1). Положим

$$
f_{ \pm}(q, 0, \varepsilon)=\alpha_{ \pm}^{-2} \widehat{\nu}_{ \pm}\left(\ln |q| / \ln \mu_{\varepsilon}, \varepsilon\right) .
$$

Функции $\widehat{\nu}_{ \pm}$периодичны по первому аргументу с периодом единица.

Далее мы предполагаем, что выполнено следующее условие.

(B) $\lim _{\varepsilon \backslash 0} \frac{\delta_{\varepsilon}}{\ln \mu_{\varepsilon}}=0$.

Вводя симплектические координаты $(x, y)$ :

$$
\begin{aligned}
& x=\frac{\ln |q|}{\ln \mathscr{M}_{\varepsilon}(q p)}=\frac{\ln |q|}{\ln \mu_{\varepsilon}}\left(1+O_{1}(q p)\right), \\
& y=\delta_{\varepsilon}^{-1} \int_{0}^{q p} \mathscr{M}_{\varepsilon}(\xi) d \xi=\delta_{\varepsilon}^{-1} q p \ln \mu_{\varepsilon}\left(1+O_{1}(q p)\right),
\end{aligned}
$$

и добавляя к ним индекс $s=\operatorname{sign}(q)$, получаем следующие формулы для сепаратрисного отображения: $\mathscr{S M}_{\varepsilon}(x, y, s)=\left(x^{+}, y^{+}, s^{+}\right)$,

$$
\left\{\begin{array}{l}
x^{+}=x+\frac{1}{\ln \mu_{\varepsilon}}\left[\ln \frac{\delta_{\varepsilon}}{\alpha_{s}^{2} \ln \mu_{\varepsilon}}+\ln \left|y^{+}+\delta_{\varepsilon} \ln \mu_{\varepsilon} \cdot O\left(1+y / \ln \mu_{\varepsilon}\right)^{2}\right|\right], \\
y^{+}=y+\ln \mu_{\varepsilon} \cdot \widehat{\nu}_{s}(x, \varepsilon)+\delta_{\varepsilon} \ln \mu_{\varepsilon} \cdot O\left(1+y / \ln \mu_{\varepsilon}\right)^{2}, \\
s^{+}=s \operatorname{sign}\left(y^{+}\right) .
\end{array}\right.
$$


Также мы предположим, что

(C) функиии $\widehat{\nu}_{ \pm}$имеют вид

$$
\widehat{\nu}_{ \pm}(\xi, \varepsilon)=\nu_{ \pm}(\xi)+o(1) \quad n p u \quad \varepsilon \rightarrow 0
$$

где функиии $\nu_{ \pm}$не обращаются тождественно в нуль.

Неравенство $\nu_{ \pm} \not \equiv 0$ является условием общего положения. Как и следовало ожидать, формулы (3.30) в “стандартной" ситуации $\mu_{0}>1, \delta_{\varepsilon}=\varepsilon, y \sim 1$ совпадают с (3.1).

\section{Глава 4. Сепаратрисное отображение Шильникова}

В этой главе мы получим формулы сепаратрисного отображения Шильникова. Это отображение определяет динамику отображения двумерной области с гиперболической неподвижной точкой только в окрестности одной гомоклинической траектории, но при этом не требуется какой-либо близости отображения к интегрируемому. Впервые подобное отображение появилось в [2], где оно использовалось для построения бесконечного множества периодических решений для произвольных двумерных отображений с гиперболической неподвижной точкой в окрестности гомоклинической траектории. Имеется ряд обобщений на многомерный случай. В главе 5 обсуждается сепаратрисное отображение в задаче Мезера, являющееся обобщением сепаратрисного отображения Шильникова.

Здесь мы рассмотрим только консервативный случай, т.е. случай симплектических диффеоморфизмов двумерной области. Нетрудно заметить, что аналогичная конструкция проходит и в общей ситуации.

\section{§1. Формулы}

Рассмотрим гладкий симплектоморфизм $T: D \rightarrow D, D \subset \mathbb{R}^{2}$, удовлетворяющий следующим условиям:

1) $T$ имеет гиперболическую неподвижную точку $\sigma$,

2) сепаратрисы $\Gamma^{u}$ и $\Gamma^{s}$ точки $\sigma$ пересекаются в точке $\gamma$.

Таким образом, система имеет гомоклиническое решение $\left\{T^{n}(\gamma)\right\}_{n \in \mathbb{Z}}, T^{n}(\gamma) \rightarrow$ $\sigma$ при $n \rightarrow \pm \infty$. Это гомоклиническое решение называется трансверсальным, если сепаратрисы $\Gamma^{u}$ и $\Gamma^{s}$ пересекаются трансверсально в точке $\gamma$. Пусть $\mu>0$ - мультипликатор неподвижной точки $\sigma$ и $\lambda=\ln \mu$.

Пусть $\Delta$ - малая окрестность точки $\gamma$ такая, что $T(\Delta) \cap \Delta=\varnothing$. Сепаратрисное отображение в рассматриваемом случае мы определим как отображение первого возвращения для области $\Delta$. Однако формулы для него удается записать лишь для меньшей области $\Delta_{1}$, в которой все точки имеют одинаковый тип возвращения в $\Delta$. А именно, точки из этой области, выходя из области $\Delta$, движутся вдоль устойчивой сепаратрисы, попадают в окрестность неподвижной точки $\sigma$ и движутся вдоль неустойчивой сепаратрисы, пока снова не попадут в $\Delta$ (см. рис. 4.1$)$.

Положим

$$
n_{r}(z)=\min \left\{n>0: T^{n}(z) \in \Delta\right\} \quad \text { и } \quad \Delta_{r}=\left\{z \in \Delta: n_{r}<+\infty\right\},
$$


т.е. множество $\Delta_{r}$ состоит из точек окрестности $\Delta$, которые под действием $T$ возвращаются в эту окрестность. Сепаратрисное отображение имеет вид:

$$
\mathscr{S} \mathscr{M}: \Delta_{r} \rightarrow \Delta, \quad \mathscr{S} \mathscr{M}(z)=T^{n_{r}}(z)
$$

ТЕОРема 4.1. Существуют (достаточно малая) окрестность $\Delta$ точки $\gamma$ и симплектические координаты $(x, y)$ такие, что

1) $\Delta=\{(x, y) \in \mathbb{T} \times \mathbb{R}: x, y \text { мальь }\}^{4}$

2) $\gamma=(x=0, y=0),\{y=0\} \subset \Gamma^{u} \cap \Delta$;

3) существует гладкое отображение $(x, y) \mapsto\left(x^{+}, y^{+}\right)^{5}$,

$$
\left\{\begin{array}{l}
x^{+}=x+x_{0}+\frac{1}{\lambda} \ln y^{+}+O_{1}\left(y^{+} \ln y^{+}\right)+O_{1}(x, y), \\
y^{+}=a x+b y+O_{2}(x, y)
\end{array}\right.
$$

которое совпадает с сепаратрисным отображением $\mathscr{S} \mathscr{M}$ на счетном наборе $\Delta_{1}$ областей, накапливающихся к кривой $\left\{y^{+}=0\right\} \subset \Gamma^{s} \cap \Delta$ :

$$
\Delta_{1}=\left\{(x, y) \in \Delta:\left(x^{+}, y^{+}\right) \in \Delta, y^{+}>0\right\},\left.\quad \mathscr{S} \mathscr{M}\right|_{\Delta_{1}}=\left.(4.1)\right|_{\Delta_{1}}
$$

здесъ $x_{0}, a, b$ - некоторье константы;

4) сепаратрисы $\Gamma^{u} u \Gamma^{s}$ пересекаются трансверсально в точке $\gamma$ тогда и только тогда, когда $a \neq 0$;

5) для $z=(x, y) \in \Delta_{1}$ количество итерачий до первого возвращения в $\Delta$

$$
n_{r}(z)=\left[-x_{0}-\frac{1}{\lambda} \ln y^{+}\right]
$$

где $[X]$ обозначает округление числа $X$ до челого ${ }^{6}$.

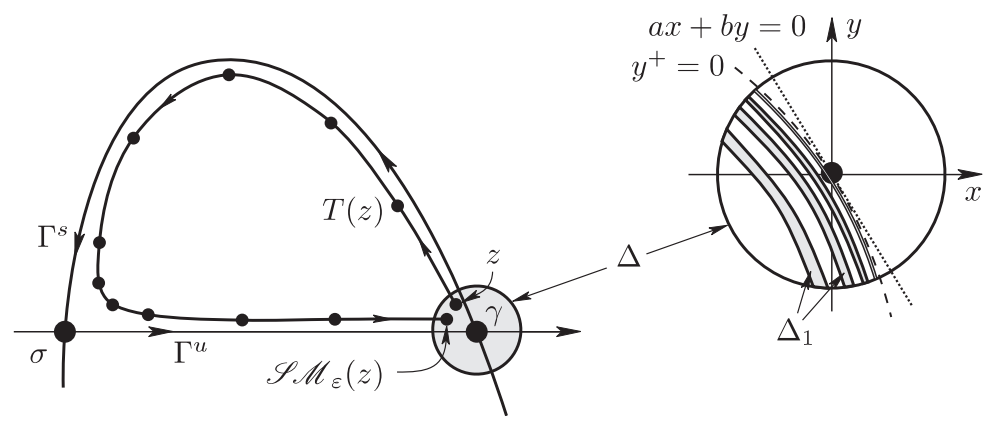

Рис. 4.1. Сепаратрисное отображение Шильникова

\footnotetext{
${ }^{4}$ Переменная $x$ - угловая, выражение: “ $x$ мало” следует понимать как близость к точке $x=0$ на окружности.

${ }^{5}$ Функция $x^{+}(x, y)$ определена при $y^{+}(x, y)>0$. Величины $x$ и $O_{1}(x, y)$ в правой части первого уравнения (4.1) имеют одинаковый порядок малости. Такой вид уравнения удобен, если считать, что $x \in \mathbb{R}$ и близок к целым числам.

${ }^{6}[X]=\left[X-\frac{1}{2}\right]$, где справа квадратные скобки обозначают обычную целую часть числа.
} 
ЗАмЕчаниЕ 4.2. (1) В случае если $T$ близко к интегрируемому, сепаратрисное отображение (4.1) совпадает с сепаратрисным отображением Заславского (3.1) в окрестности гомоклинической точки $\left\{(x, y): y=0, y^{+}=0\right\}$.

(2) Множество $\Delta_{1}$ не инвариантно относительно сепаратрисного отображения и, вообще говоря, не содержит инвариантных подобластей. Траектории, описываемые отображением (4.1), определены лишь на канторовом множестве $\bigcap_{n \in \mathbb{Z}} \mathscr{S}^{n}\left(\Delta_{1}\right)$ меры нуль.

\section{§ 2. Доказательство}

Согласно теореме 2.1, в малой окрестности гиперболической неподвижной точки можно ввести нормальные координаты $(q, p)$, в которых $T$ имеет вид

$$
\mathscr{T}:(q, p) \mapsto(q \mathscr{M}(p q), p / \mathscr{M}(p q))
$$

и $\sigma=(q=0, p=0)$. Как и в $\S 2$ главы 3 , эти координаты можно продолжить вдоль сепаратрис, так что в продолженных координатах $T$ также имеет вид (4.2). Тогда в окрестности точки $\gamma$ имеется, по крайней мере, две системы координат $\left(q_{u}, p_{u}\right)$ и $\left(q_{s}, p_{s}\right)$, полученные продолжением нормальных координат вдоль неустойчивой $\Gamma^{u}$ и устойчивой $\Gamma^{s}$ сепаратрис соответственно. Пусть

$$
\gamma=\left(q_{u}=q_{0}, p_{u}=0\right)=\left(q_{s}=0, p_{s}=p_{0}\right), \quad q_{0}, p_{0}=\text { const. }
$$

Без ограничения общности можно считать $q_{0}, p_{0}>0$. Эти координаты отождествляются отображением склейки $\mathscr{G}$ :

$$
\mathscr{G}:\left\{\left(q_{u}, p_{u}\right): q_{u}-q_{0}, p_{u} \text { малы }\right\} \rightarrow\left\{\left(q_{s}, p_{s}\right): q_{s}, p_{s}-p_{0} \text { малы }\right\} .
$$

Имеем $\mathscr{G}\left(q_{u}=q_{0}, p_{u}=0\right)=\left(q_{s}=0, p_{s}=p_{0}\right)$, следовательно,

$$
\begin{gathered}
\left(q_{s}, p_{s}\right)=\mathscr{G}\left(q_{u}, p_{u}\right), \\
\left\{\begin{array}{l}
q_{s}=O_{1}\left(q_{u}-q_{0}, p_{u}\right)=\widehat{a}\left(q_{u}-q_{0}\right)+\widehat{b} p_{u}+O_{2}\left(q_{u}-q_{0}, p_{u}\right), \\
p_{s}=p_{0}+O_{1}\left(q_{u}-q_{0}, p_{u}\right),
\end{array}\right.
\end{gathered}
$$

где $\widehat{a}$ и $\widehat{b}-$ некоторые константы.

ПРЕДЛОЖенИЕ 4.3. Сепаратрисъ $\Gamma^{u} u \Gamma^{s}$ пересекаются трансверсально в точке $\gamma$ тогда и только тогда, когда $\widehat{a} \neq 0$.

ДокАЗАтеЛЬство. Рассмотрим в окрестности точки $\gamma$ отрезки сепаратрис $\Gamma^{s}$ и $\Gamma^{u}$, на которых лежит эта точка. Тогда в координатах $\left(q_{s}, p_{s}\right)$ первый задается равенством $q_{s}=0$, а второй $\left(p_{u}=0\right)$ задается параметрически:

$$
q_{s}=\widehat{a}\left(q_{u}-q_{0}\right)+O_{2}\left(q_{u}-q_{0}\right), \quad p_{s}=p_{0}+O_{1}\left(q_{u}-q_{0}\right) .
$$

Следовательно, касательные к этим кривым не совпадают тогда и только тогда, когда $\widehat{a} \neq 0$. 
Сепаратрисное отображение получается из отображения склейки (4.4) если в $\mathbb{R}_{q, p}^{2}$ отождествить координаты точек $\left(q_{s}, p_{s}\right)$ и $\left(q_{u}, p_{u}\right)$, связанные соотношениями

$$
q_{u}=\mathscr{M}^{n_{r}} q_{s}, \quad p_{u}=\mathscr{M}^{-n_{r}} p_{s}
$$

Введем следующие симплектические координаты:

$$
\begin{aligned}
& y=\int_{0}^{q p} \ln \mathscr{M}(\xi) d \xi=\lambda q p+O_{2}(q p), \\
& x=\frac{\ln \left(q / q_{0}\right)}{\ln \mathscr{M}}=\frac{\ln \left(q / q_{0}\right)}{\lambda}\left(1+O_{1}(q p)\right) .
\end{aligned}
$$

Будем обозначать $\left(x_{u}, y_{u}\right)$ и $\left(x_{s}, y_{s}\right)$ координаты, соответствующие координатам $\left(q_{u}, p_{u}\right)$ и $\left(q_{s}, p_{s}\right)$. Сепаратрисное отображение записывается в координатах $\left(x_{u}, y_{u}\right)$, в которых $\Delta=\left\{\left(x_{u}, y_{u}\right): x_{u}, y_{u}\right.$ малы $\}$.

В переменных $(x, y)$ отождествление (4.5) принимает вид $x_{u}=x_{s} \bmod 1$. Для доказательства теоремы 4.1 остается записать отображение склейки в переменных $(x, y)$ и затем положить $x$ определенным по модулю 1.

Обращая равенства (4.6), имеем:

$$
q p=\frac{1}{\lambda} y+O_{2}(y), \quad q=q_{0} e^{\lambda x\left(1+O_{1}(y)\right)} .
$$

Пусть $\left(x_{s}, y_{s}\right)=\mathscr{G}\left(x_{u}, y_{u}\right),\left(x_{u}, y_{u}\right) \in\left\{q_{u}-q_{0}, p_{u}\right.$ малы $\}$. Тогда, используя (4.4), последовательно получаем

$$
\begin{aligned}
q_{u}-q_{0} & =\lambda q_{0} x_{u}+O_{2}\left(x_{u}, y_{u}\right), & p_{u} & =\frac{1}{\lambda q_{0}} y_{u}+O_{2}\left(x_{u}, y_{u}\right), \\
p_{s} & =p_{0}+O_{1}\left(x_{u}, y_{u}\right), & q_{s} & =\frac{1}{\lambda p_{0}} y_{s}\left(1+O_{1}\left(x_{u}, y_{u}\right)\right), \\
y_{s} & =a x_{u}+b y_{u}+O_{2}\left(x_{u}, y_{u}\right), & x_{s} & =\frac{\ln \left(q_{s} / q_{0}\right)}{\lambda}\left(1+O_{1}\left(y_{s}\right)\right),
\end{aligned}
$$

где $a=\lambda^{2} p_{0} q_{0} \widehat{a} \neq 0, b=\lambda p_{0} \widehat{b} / q_{0}$. Имеем:

$$
\begin{aligned}
& x_{s}=x_{0}+\frac{1}{\lambda} \ln y_{s}+O\left(y_{s} \ln y_{s}\right)+O_{1}\left(x_{u}, y_{u}\right), \\
& y_{s}=a x_{u}+b y_{u}+O_{2}\left(x_{u}, y_{u}\right),
\end{aligned}
$$

где $x_{0}=\frac{1}{\lambda} \ln \frac{1}{\lambda p_{0} q_{0}}$.

Количество итераций точки с координатами $\left(x_{u}, y_{u}\right)$ до первого возвращения в $\Delta$ определяется из условия: $x_{s}+n_{r}-$ мало. Отсюда получим $n_{r}\left(x_{u}, y_{u}\right)=$ $\left[-x_{0}-\frac{1}{\lambda} \ln y_{s}\right]$

Теперь, рассматривая $x$ как угловую переменную по модулю 1 , окончательно получаем $\left(x^{+}, y^{+}\right)=\mathscr{S} \mathscr{M}(x, y)$,

$$
\left\{\begin{array}{l}
x^{+}=x+a_{0}+\frac{1}{\lambda} \ln y^{+}+O\left(y^{+} \ln y^{+}\right)+O_{1}(x, y), \\
y^{+}=a x+b y+O_{2}(x, y) .
\end{array}\right.
$$

Теорема 4.1 доказана. 


\section{Глава 5. Сепаратрисное отображение в задаче Мезера}

В этой главе мы получим формулы для сепаратрисного отображения задачи Мезера (см. §4 главы 1). В главе 9 мы воспользуемся этими формулами для доказательства теоремы 1.5 .

\section{§ 1. Определение}

Рассмотрим гамильтонову систему $(\mathscr{M}, \Omega, \mathscr{H})$ с двумя с половиной степенями свободы, где $\mathscr{M}$ - гладкое симплектическое многообразие, $\operatorname{dim} \mathscr{M}=4, \Omega-$ симплектическая структура, гамильтониан $\mathscr{H} \in C^{\mathbf{r}+1}(\mathscr{M} \times \mathbb{T}), \mathbf{r} \geqslant 13$, имеет вид:

$$
\mathscr{H}(\mathscr{Z}, t)=\mathscr{H}_{0}(\mathscr{Z})+\mathscr{H}_{1}(\mathscr{Z}, t), \quad \mathscr{Z} \in \mathscr{M}, \quad t \in \mathbb{T}=\mathbb{R} / \mathbb{Z} .
$$

Пусть $\mathscr{G}^{\mu}: \mathscr{M} \rightarrow \mathscr{M}$ - однопараметрическая группа диффеоморфизмов фазового пространства. Предположим, что

$$
\mathscr{H}_{0} \circ \mathscr{G}^{\mu}=e^{2 \mu} \mathscr{H}_{0}, \quad \mathscr{H}_{1} \circ \mathscr{G}^{\mu}=\mathscr{H}_{1}, \quad\left(\mathscr{G}^{\mu}\right)^{*} \Omega=e^{\mu} \Omega .
$$

Ниже предполагаем, что $\left\{\mathscr{H}_{0}=1\right\} \neq \varnothing$.

ОПредеЛЕНиЕ 5.1. Функцию $\mathscr{F}: \mathscr{M} \rightarrow \mathbb{R}$ будем называть $\mathscr{G}^{\mu}$-однородной степени $k$, если

$$
\mathscr{F} \circ \mathscr{G}^{\mu}=e^{k \mu} \mathscr{F} .
$$

Таким образом, функции $\mathscr{H}_{0}$ и $\mathscr{H}_{1}$ являются $\mathscr{G}^{\mu}$-однородными степени 2 и 0 соответственно. В задаче Мезера функции $\mathscr{H}_{0}$ и $\mathscr{H}_{1}-$ кинетическая и потенциальная энергии соответственно, а группа $\mathscr{G}^{\mu}$ - гомотетия по импульсам:

$$
\mathscr{G}^{\mu}(q, p)=\left(q, e^{\mu} p\right), \quad \mathscr{Z}=(q, p) .
$$

Автономную гамильтонову систему с гамильтонианом $\mathscr{H}_{0}$ будем называть невозмущенной, а исходную - возмущенной.

Предположим, что невозмущенная система имеет гиперболическую периодическую траекторию $\sigma \subset \mathscr{M}$ и гомоклиническое к ней решение $\gamma: \mathbb{R} \rightarrow \mathscr{M}$.

Поверхности уровня гамильтониана $\mathscr{H}_{0}$ инвариантны относительно фазового потока $\Phi_{0}^{t}$ невозмущенной системы. Обозначим их

$$
\mathscr{M}_{\mu}=\left\{\mathscr{Z} \in \mathscr{M}: \mathscr{H}_{0}(\mathscr{Z})=e^{2 \mu}\right\} .
$$

Будем считать, что гиперболическая траектория $\sigma$, а значит, и ее гомоклиника $\gamma$ лежат на единичном уровне $\mathscr{M}_{0}$.

Динамика невозмущенной системы "одинакова" на каждом уровне гамильтониана $\mathscr{H}_{0}$. Точнее, имеет место следующая лемма.

ЛЕмма 5.2.

$$
\Phi_{0}^{t} \circ \mathscr{G}^{\mu}=\mathscr{G}^{\mu} \circ \Phi_{0}^{e^{\mu} t} .
$$

ДокАзАТЕЛьство. Каждый уровень гамильтониана инвариантен относительно фазового потока и

$$
\mathscr{G}^{\mu *}\left(\Omega-d \mathscr{H}_{0} \wedge d t\right)=e^{\mu} \Omega-e^{2 \mu} d \mathscr{H}_{0} \wedge d t=e^{\mu}\left(\Omega-d \mathscr{H}_{0} \wedge d e^{\mu} t\right) .
$$


Таким образом, получим семейство гиперболических решений и гомоклинических к ним решений

$$
\sigma_{\mu}(t)=\mathscr{G}^{\mu} \sigma\left(e^{\mu} t\right), \quad \gamma_{\mu}(t)=\mathscr{G}^{\mu} \gamma\left(e^{\mu} t\right) .
$$

Мы будем исследовать динамику в окрестности этого семейства гомоклинических решений для достаточно больших $\mu>0$. В расширенном фазовом пространстве $\mathscr{M} \times \mathbb{T}_{t}$ выберем гиперповерхность $\mathscr{Q}$, трансверсальную гамильтонову векторному полю $v_{\mathscr{H}}+\partial_{t}$ и содержащую двумерное многообразие

$$
\left\{\left(\gamma_{\mu}(0), t\right) \in \mathscr{M} \times \mathbb{R}_{t}: \mu^{-1} \text { мало }\right\}
$$

Сепаратрисное отображение $\mathscr{S} \mathscr{M}$ определим как отображение Пуанкаре этой четырехмерной поверхности $\mathscr{Q}$. Точнее, выберем произвольную точку $\mathscr{Z}$ на этой поверхности. Пусть

$$
t_{r}(\mathscr{Z})=\min \left\{t>0: \Phi^{t} \mathscr{Z} \in \mathscr{Q}\right\} \quad \text { и } \quad \mathscr{Q}_{r}=\left\{\mathscr{Z} \in \mathscr{Q}: t_{r}<+\infty\right\},
$$

т.е. множество $\mathscr{Q}_{r}$ состоит из точек на поверхности $\mathscr{Q}$, которые под действием фазового потока возвращаются на эту поверхность. Тогда определение сепаратрисного отображения выглядит следующим образом:

$$
\mathscr{S} \mathscr{M}: \mathscr{Q}_{r} \rightarrow \mathscr{Q}, \quad \mathscr{S} \mathscr{M}(\mathscr{Z})=\Phi^{t_{r}} \mathscr{Z} .
$$

Ниже мы используем вместо $\mathscr{Q}_{r}$ меньшую область $\mathscr{Q}_{*}$, которая лежит в окрестности множества $(5.4)$ и ограничение $\mathscr{S} \mathscr{M}$ на которую является гладким отображением.

\section{§ 2. Формулы}

Без ограничения общности предполагаем, что среднее функции $\mathscr{H}_{1}(\cdot, t)$ на гиперболической траектории нулевое для любого $t \in \mathbb{T}$, т.е.

$$
\int_{0}^{l} \mathscr{H}_{1}(\sigma(\tau), t) d \tau=0
$$

где $l-$ период $\sigma(t): \sigma(t)=\sigma(t+l)$. Существуют числа $t^{u, s} \bmod l$ такие, что $\gamma(t) \rightarrow \sigma\left(t+t^{u}\right)$ при $t \rightarrow-\infty$ и $\gamma(t) \rightarrow \sigma\left(t+t^{s}\right)$ при $t \rightarrow+\infty$. Поскольку $\gamma(t)$ экспоненциально стремится к $\sigma$ при $t \rightarrow \pm \infty$, то следующий предел

$$
I(t)=\lim _{T \rightarrow+\infty}\left[\int_{-T}^{T} \mathscr{H}_{1}(\gamma(\tau), t) d \tau-\int_{-T+t^{u}}^{T+t^{s}} \mathscr{H}_{1}(\sigma(\tau), t) d \tau\right]
$$

существует и не зависит от выбора чисел $t^{u, s}$. Периодическая функция $I(t)$ называется интегралом Пуанкаре-Мельникова. Также обозначим

$$
I^{\prime}(t)=\frac{d}{d t} I(t)
$$

Пусть $\nu=1 / l$ - частота и $\lambda$ - положительный показатель Ляпунова решения $\sigma(t)$. Положим $x_{0}=\nu\left(t^{s}-t^{u}\right)$. Число $x_{0}$ определено по модулю 1 и не зависит от выбранных параметризаций решений. 
ОПРЕДЕЛЕНИЕ 5.3. Пусть $D$ - область в $\mathbb{R}^{n}\left(x_{1}, \ldots, x_{n}\right)$. Пусть $R, r_{1}, \ldots$, $r_{m}: D \rightarrow \mathbb{R}$ - гладкие функции на ней. Будем говорить, что

$$
R=\boldsymbol{O}_{1}\left(r_{1}, \ldots, r_{m}\right) \text { на области } D \text { в координатах }\left(x_{1}, \ldots, x_{n}\right),
$$

если $R$ представляется в виде:

$$
R=\sum_{j} a_{j} r_{j}, \quad\left|a_{j}\right|<C, \quad\left|\frac{\partial a_{j}}{\partial x_{k}}\right|<L, \quad j=1, \ldots, m, \quad k=1, \ldots, n,
$$

т.е. гладкие функции $a_{j}: D \rightarrow \mathbb{R}$ имеют конечные $C^{1}$-нормы на $D$. Аналогичным образом определим символ $\boldsymbol{O}_{2}\left(r_{1}, \ldots, r_{m}\right)$ :

$$
R=\boldsymbol{O}_{2}\left(r_{1}, \ldots, r_{m}\right) \Leftrightarrow R=\sum_{i, j} a_{i j} r_{i} r_{j}, \quad\left|a_{i j}\right|<C, \quad\left|\frac{\partial a_{i j}}{\partial x_{k}}\right|<L .
$$

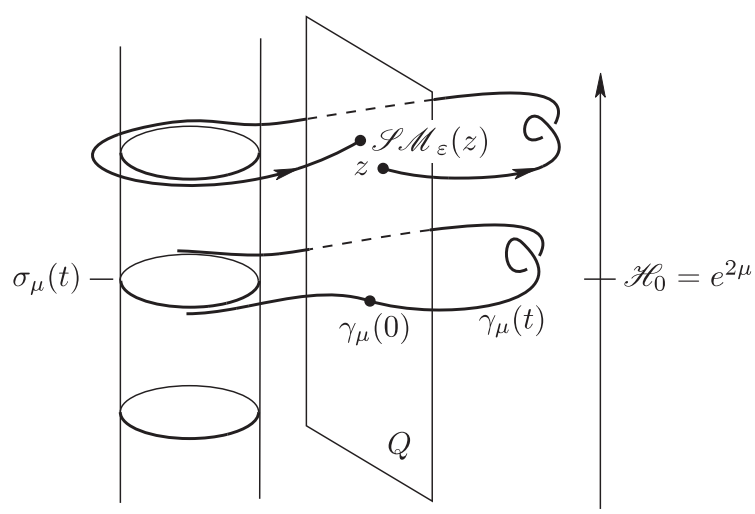

Рис. 5.1. Сепаратрисное отображение в задаче Мезера

ТЕОРема 5.4 [25]. На поверхности $\mathscr{Q}$ существуют координаты $(x, y, t, h)$ :

$$
\mathscr{Q}=\left\{(x, y, t, h) \in \mathbb{T} \times \mathbb{R}^{3}: y, h^{-1}-\mathcal{м a л b \iota}\right\}
$$

такие, что

1) $t$ совпадает с исходным временем;

2) $\mathscr{H}_{0}=h+\boldsymbol{O}(1)$ на $\mathscr{Q}$

3) на области ${ }^{7}\left\{\frac{1}{\sqrt[4]{h}}<y ; x, y\right.$ мальь $\} \subset \mathscr{Q}$ существует гладкая функиия

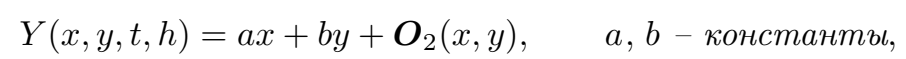

такая, что

$$
\mathscr{Q}_{*}=\left\{(x, y, t, h) \in \mathscr{Q}: \frac{1}{\sqrt[4]{h}}<y, \frac{1}{\sqrt[4]{h}}<Y ; x, y \text { мальь }\right\}
$$

\footnotetext{
${ }^{7}$ Переменная $x$ - угловая и выражение “ $x$ мало" следует понимать как близость к точке $x=0$ на окружности.
} 
u сепаратрисное отображение имеет вид

$$
\begin{aligned}
\mathscr{S} \mathscr{M}: \mathscr{Q}_{*} \rightarrow \mathscr{Q}, & \mathscr{S} \mathscr{M}(x, y, t, h)=\left(x^{+}, \rho^{+}, t^{+}, h^{+}\right), \\
& \begin{cases}x^{+}=x+x_{0}-\frac{\nu}{\lambda} \ln Y+\boldsymbol{O}_{1}, \\
y^{+}=Y & +Y \boldsymbol{O}_{1}, \\
t^{+}=t-\frac{1}{\lambda \sqrt{h}} \ln Y & +\frac{1}{\sqrt{h}} \boldsymbol{O}_{1}, \\
h^{+}=h+\frac{1}{\sqrt{h}} I^{\prime}(t) & +\frac{1}{\sqrt{h}} \boldsymbol{O}_{1},\end{cases}
\end{aligned}
$$

где $\boldsymbol{O}_{1}=\boldsymbol{O}_{1}(Y \ln Y)+\boldsymbol{O}_{1}(x, y)$ на $\mathscr{Q}_{*} ;$

4) гомоклиническое решение невозмущенной системъ $\gamma(t)$ трансверсалъно тогда и только тогда, когда $a \neq 0$.

ЗАмЕЧАНИЕ 5.5. Для сравнения приведем формулы сепаратрисного отображения для невозмущенной системы $\left(\mathscr{M}, \mathscr{H}_{0}, \Omega\right)$.

На самом деле координаты $(x, y, t, h)$ из теоремы 5.4 являются малой деформацией при $h \rightarrow+\infty$ координат $(\widetilde{x}, \widetilde{y}, t, \widetilde{h})$ таких, что $\mathscr{Q} \subset \widetilde{\mathscr{Q}}=\{(\widetilde{x}, \widetilde{y}, t, \widetilde{h}) \in$ $\mathbb{T} \times \mathbb{R}^{3}: \widetilde{y}$ мало\} и выполнены следующие свойства:

1) $t$ - время;

2) $\mathscr{H}_{0}=\widetilde{h}$;

3) $\left(\gamma_{\mu}(0), t\right)=\left(\widetilde{x}=0, \widetilde{y}=0, t, \widetilde{h}=e^{2 \mu}\right)$;

4) на множестве $\{\widetilde{x}, \widetilde{y}$ малы $\} \subset \widetilde{\mathscr{Q}}$ существует функция $\widetilde{Y}(\widetilde{x}, \widetilde{y}, t, \widetilde{h})=a \widetilde{x}+$ $b \widetilde{y}+\boldsymbol{O}_{2}(\widetilde{x}, \widetilde{y})$ такая, что

$$
\widetilde{\mathscr{Q}}_{*}=\{(\widetilde{x}, \widetilde{y}, t, \widetilde{h}) \in \mathscr{Q}: \widetilde{Y}>0 ; \widetilde{x}, \widetilde{y} \text { малы }\}
$$

и сепаратрисное отображение невозмущенной системы имеет вид

$$
\begin{aligned}
\mathscr{S}_{0}: \widetilde{\mathscr{Q}}_{*} \rightarrow \widetilde{\mathscr{Q}}, & \mathscr{S}_{\mathscr{M}_{0}}(\widetilde{x}, \widetilde{y}, t, \widetilde{h})=\left(x^{+}, y^{+}, t^{+}, h^{+}\right), \\
& \left\{\begin{array}{l}
x^{+}=\widetilde{x}+x_{0}-\frac{\nu}{\lambda} \ln \tilde{Y}+\boldsymbol{O}_{1}, \\
y^{+}=\tilde{Y} \\
t^{+}=t-\frac{1}{\lambda \sqrt{\tilde{h}}} \ln \tilde{Y} \quad+\frac{1}{\sqrt{\tilde{h}}} \boldsymbol{O}_{1}, \\
h^{+}=\tilde{h},
\end{array}\right.
\end{aligned}
$$

где $\boldsymbol{O}_{1}=\boldsymbol{O}_{1}(\widetilde{Y} \ln \tilde{Y})+\boldsymbol{O}_{1}(x, y)$ на $\widetilde{\mathscr{Q}}_{*}$ и не зависят от $t$ и $\widetilde{h}$.

Таким образом, первые два уравнения системы (5.10) отделяются. Нетрудно видеть, что эти уравнения совпадают с уравнениями сепаратрисного отображения Шильникова (теорема 4.1). 


\section{Глава 6. Сепаратрисное отображение в априори неустойчивых системах}

\section{$\S 1$. Описание конструкции}

Начнем с конструкции сепаратрисного отображения. Далее мы пользуемся обозначениями, введенными в § 5 главы 1. Тор (1.12) инвариантен относительно невозмущенной системы и называется гиперболическим [49], [50] (правильнее говорить - частично гиперболическим). Имеется два асимптотических многообразия

$$
\begin{gathered}
\widehat{\Gamma}^{+}\left(y^{0}\right), \widehat{\Gamma}^{-}\left(y^{0}\right) \subset\left\{(y, x, v, u, t): y=y^{0}, H_{0}\left(y^{0}, v, u\right)=H_{0}\left(y^{0}, 0,0\right)\right\} \\
\widehat{\Gamma}^{ \pm}\left(y^{0}\right)=\left\{y^{0}\right\} \times \mathbb{T}^{n} \times \widehat{\gamma}^{ \pm}\left(y^{0}\right) \times \mathbb{T}
\end{gathered}
$$

Многообразия $\widehat{\Gamma}^{ \pm}\left(y^{0}\right)$ состоят из невозмущенных решений, стремящихся к $N\left(y^{0}\right)$ при $t \rightarrow \pm \infty$.

Пусть $\mathscr{D} \subset \mathscr{D}_{0}$ - открытая связная область с компактным замыканием $\overline{\mathscr{D}}$. Далее мы рассматриваем динамику в возмущенной системе в окрестности множества

$$
\widehat{\Gamma}=\bigcup_{y \in \bar{D}}\left(\widehat{\Gamma}^{+}(y) \cup \widehat{\Gamma}^{-}(y)\right) .
$$

В этой окрестности содержится наиболее интересная часть возмущенной динамики. Удобно перейти к отображению за единичное время $T_{\varepsilon}$, переводящему точку $(y, x, v, u)$ в точку $(y(1), x(1), v(1), u(1))$, где $(y(t), x(t), v(t), u(t))$ - решение системы (1.11) с начальными условиями $(y(0), x(0), v(0), u(0))=(y, x, v, u)$.

Отображение $T_{0}$ имеет $n$-мерные гиперболические торы $L(y)=\operatorname{pr}(N(y))$, где pr: $(y, x, v, u, t) \mapsto(y, x, v, u)-$ проекция. Пусть $\Sigma^{ \pm}(y)=\operatorname{pr}\left(\widehat{\Gamma}^{ \pm}(y)\right)-$ асимптотические многообразия. Определим сепаратрисное отображение $\mathscr{S}_{\mathscr{M}}$, соответствующее $T_{\varepsilon}$ в окрестности множества

$$
\Sigma=\bigcup_{y \in \bar{D}}\left(\Sigma^{+}(y) \cup \Sigma^{-}(y)\right)=\operatorname{pr}(\widehat{\Gamma}) .
$$

Пусть $U$ - малая окрестность множества $\bigcup_{y \in \overline{\mathscr{D}}} L(y)$ и $\mathbf{U}$ - окрестность $\Sigma$ (см. рис. 1.3 на с. 15 , где $\Sigma$ изображено в случае $n=0)$. Если $\mathbf{U}$ достаточно мала, то $\mathbf{U} \backslash U$ состоит из двух компонент связности $\mathbf{U}^{+}$и $\mathbf{U}^{-}$, так что $\Sigma^{ \pm} \subset \mathbf{U}^{ \pm} \cup U$.

Рассмотрим точку $z \in \mathbf{U}^{+} \cup \mathbf{U}^{-}$. Пусть $k_{1}=k_{1}(z)$ - минимальное натуральное число такое, что $T_{\varepsilon}^{k_{1}}(z) \notin \mathbf{U}^{+} \cup \mathbf{U}^{-}$, и пусть $k_{2}=k_{2}(z)$ - минимальное натуральное число такое, что $k_{2}>k_{1}$ и $T_{\varepsilon}^{k_{2}}(z) \in \mathbf{U}^{+} \cup \mathbf{U}^{-}$. При $k=k_{1}$ траектория $T_{\varepsilon}^{k}(z)$ покидает $\mathbf{U}^{+} \cup \mathbf{U}^{-}$. При $k=k_{2}$ траектория возвращается в $\mathbf{U}^{+} \cup \mathbf{U}^{-}$. Назовем точку $z$ хорошей, если $k_{2}<\infty$ и $T_{\varepsilon}^{k_{1}}(z), \ldots, T_{\varepsilon}^{k_{2}-1}(z) \in U$. Полагая

$$
\mathbf{U}_{\varepsilon}=\left\{z \in \mathbf{U}^{+} \cup \mathbf{U}^{-}: z-\text { хорошая }\right\}
$$

получаем отображения

$$
\mathscr{S} \mathscr{M}_{\varepsilon}\left(\cdot, k_{2}(\cdot)+k\right): \mathbf{U}_{\varepsilon} \rightarrow \mathbf{U}^{+} \cup \mathbf{U}^{-}, \quad \mathscr{S}_{\mathscr{M}_{\varepsilon}}\left(z, k_{2}(z)+k\right)=T_{\varepsilon}^{k_{2}(z)+k}(z) .
$$

Здесь $k \in\{0,1, \ldots\}$ - параметр и предполагается, что $T^{k_{2}+1}(z), \ldots, T^{k_{2}+k}(z) \in$ $\mathbf{U}^{+} \cup \mathbf{U}^{-}$. Далее положим $t_{+}=k_{2}(z)+k$. 


\section{§ 2. Формулы для сепаратрисного отображения}

Положим $E(y)=H_{0}(y, 0,0), \nu=E_{y}: \mathscr{D}_{0} \rightarrow \mathbb{R}^{n}$. Тогда $(\nu(y), 1) \in \mathbb{R}^{n+1}-$ вектор частот на невозмущенном торе $N(y)$. Функцию $H_{1}(y, x, 0,0, t)$ можно разложить в ряд Фурье:

$$
H_{1}(y, x, 0,0, t)=\sum_{\left(k, k_{0}\right) \in \mathbb{Z}^{n} \times \mathbb{Z}} H_{1}^{k, k_{0}}(y) e^{2 \pi i\left(\langle k, x\rangle+k_{0} t\right)} .
$$

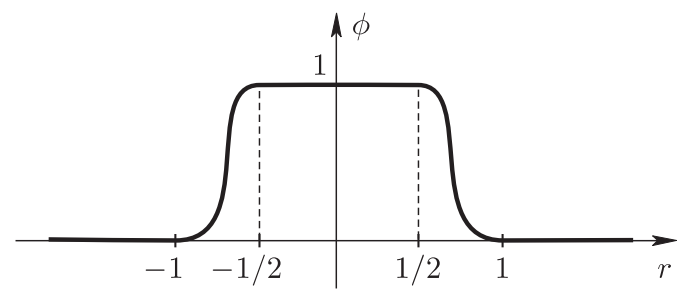

Рис. 6.1. Функция $\phi(r)$

Пусть $\phi: \mathbb{R} \rightarrow[0,1]-C^{\infty}$-гладкая функция такая, что $\phi(r)=0$ для любого $|r| \geqslant 1$ и $\phi(r)=1$ для любого $|r| \leqslant 1 / 2$ (см. рис. 6.1). Положим

$$
\begin{aligned}
\overline{\mathbf{H}}(y, x, t) & =\sum_{\left(k, k_{0}\right) \in \mathbb{Z}^{n} \times \mathbb{Z}} \phi\left(\frac{\langle k, \nu(y)\rangle+k_{0}}{\varepsilon^{1 / 4}}\right) H_{1}^{k, k_{0}}(y) e^{2 \pi i\left(\langle k, x\rangle+k_{0} t\right)}, \\
\mathbf{H}(y, x) & =\overline{\mathbf{H}}(y, x, 0) .
\end{aligned}
$$

Функция $\overline{\mathbf{H}}$ - сглаженное среднее от $H_{1}(y, x, 0,0, t)$ вдоль невозмущенных траекторий на торах $N(y)$. Она поточечно стремится к обычному среднему

$$
\sum_{\langle k, \nu(y)\rangle+k_{0}=0} H_{1}^{k, k_{0}}(y) e^{2 \pi i\left(\langle k, x\rangle+k_{0} t\right)}=\lim _{T \rightarrow \infty} \frac{1}{T} \int_{0}^{T} H_{1}(y, x+\nu(y) s, 0,0, t+s) d s
$$

при $\varepsilon \rightarrow 0$. Мы предпочитаем иметь дело с $\mathbf{H}$ и $\overline{\mathbf{H}}$, так как функция (6.3) разрывна в резонансных $y$.

Пусть $K$ - компакт. Определим следующие нормы в пространствах $C^{\mathbf{r}}(\overline{\mathscr{D}} \times$ $K)$. При $f \in C^{\mathbf{r}}(\overline{\mathscr{D}} \times K)$

$$
\|f(y, z)\|_{\mathbf{r}}^{(b)}=\max _{0 \leqslant l^{\prime}+l^{\prime \prime} \leqslant \mathbf{r}} b^{l^{\prime}}\left|\frac{\partial^{l^{\prime}+l^{\prime \prime}} f}{\partial y_{1}^{l_{1}^{\prime}} \ldots \partial y_{n}^{l_{n}^{\prime}} \partial z_{1}^{l_{1}^{\prime \prime}} \ldots \partial z_{m}^{l_{m}^{\prime \prime}}}\right|,
$$

где $l^{\prime}=l_{1}^{\prime}+\cdots+l_{n}^{\prime}$ и $l^{\prime \prime}=l_{1}^{\prime \prime}+\cdots+l_{m}^{\prime \prime}$. Считается, что $f$ может принимать значения в $\mathbb{R}^{s}$, где $s$ - произвольное натуральное число. Нормы $\|\cdot\|_{\mathbf{r}}^{(b)}$ анизотропны: переменные $y$ играют для них особую роль, так как производным по $y$ в (6.4) соответствуют дополнительные множители $b$. Очевидно, $\|\cdot\|_{\mathbf{r}}^{(1)}-$ обычная $C^{\mathbf{r}}$-норма.

При $f \in C^{\mathbf{r}}(\overline{\mathscr{D}} \times K)$ и $g \in C^{0}(\overline{\mathscr{D}} \times K)$ скажем, что

$$
f=O_{k}^{(b)}(g), \text { если }\|f\|_{\mathbf{r}}^{(b)} \leqslant C|g|^{k},
$$


где $C$ не зависит от $b$. Обозначим для краткости

$$
\|\cdot\|_{\mathbf{r}}^{*}=\|\cdot\|_{\mathbf{r}}^{\left(\varepsilon^{1 / 4}\right)}, \quad O^{(b)}=O_{1}^{(b)} \quad \text { и } \quad O_{k}^{*}=O_{k}^{\left(\varepsilon^{1 / 4}\right)} .
$$

Далее в зависимости от контекста роль “особой” переменной $y$ в $\|\cdot\|_{\mathbf{r}}^{*}$ и $O_{k}^{*}$ может играть $y^{+}, \eta$ или $\eta^{+}$.

Согласно (6.2) и условию $\mathbf{H}_{\mathbf{0}} \mathbf{1}$ (с. 15), имеем: $\mathbf{H}=O^{*}(1)$.

Функция $\overline{\mathbf{H}}$ обладает следующим свойством:

$$
\overline{\mathbf{H}}\left(y, x+\nu(y) t, t_{0}+t\right)=\overline{\mathbf{H}}\left(y, x, t_{0}\right)+O^{*}\left(\varepsilon^{1 / 4} t\right) .
$$

Полагая $t_{0}, t \in \mathbb{Z}$, получаем:

$$
\mathbf{H}(y, x+\nu(y) l)=\mathbf{H}(y, x)+O^{*}\left(\varepsilon^{1 / 4} l\right), \quad l \in \mathbb{Z} .
$$

$\mathrm{B}$ частности, $\mathbf{H}(y, x+\nu \tau)$ периодична по $x$ и приближенно периодична по $\tau$.

ТеОрема 6.1. Предположим, что выполнены условия $\mathbf{H}_{\mathbf{0}} \mathbf{1}-\mathbf{H}_{\mathbf{0}} \mathbf{4}$. Тогда существуют гладкие функиии

$$
\lambda, \kappa^{ \pm}: \overline{\mathscr{D}} \rightarrow \mathbb{R}, \quad \Theta^{ \pm}: \overline{\mathscr{D}} \times \mathbb{T}^{n+1} \rightarrow \mathbb{R}
$$

и координатъ $(\eta, \xi, h, \tau)$ такие, что выполнень следующие условия:

1) $\omega=d \eta \wedge d \xi+d h \wedge d \tau$,

2) $\eta=y+O^{*}\left(\varepsilon^{3 / 4}, H_{0}-E(y)\right), \xi+\nu(\eta) \tau=x+f$, где функиия $f$ зависит только от $(y, u, v, \varepsilon)$ и такова, что $f(y, 0,0,0)=0, h=H_{0}+O^{*}\left(\varepsilon^{3 / 4}, H_{0}-E(y)\right)$, $H_{0}=H_{0}(y, u, v)$.

Положим

$$
w=h^{+}-E\left(\eta^{+}\right)-\varepsilon \mathbf{H}\left(\eta^{+}, \xi+\nu\left(\eta^{+}\right) \tau\right) .
$$

3) Для любых $\left(\eta^{+}, \xi, h^{+}, \tau\right)$ таких, что

$$
\begin{gathered}
c^{-1} \varepsilon^{5 / 4}|\ln \varepsilon|<|w|<c \varepsilon^{7 / 8}, \\
|\tau|<c^{-1}, \quad c<|w| e^{\lambda\left(\eta^{+}\right) t^{+}}<c^{-1},
\end{gathered}
$$

отображение за время $t^{+} g_{\varepsilon}^{t^{+}} \equiv T_{\varepsilon}^{t^{+}}=\mathscr{S}_{\varepsilon}$ задается следующим образом: $\mathscr{S} \mathscr{M}_{\varepsilon}\left(\eta, \xi, h, \tau, s, t^{+}\right)=\left(\eta^{+}, \xi^{+}, h^{+}, \tau^{+}, s^{+}\right)$,

$$
\begin{aligned}
\eta^{+} & =\eta-\varepsilon \Theta_{\xi}^{s}\left(\eta^{+}, \xi, \tau\right)-\frac{w_{\xi}}{\lambda} \ln \left|\frac{\kappa^{s} w}{\lambda}\right|+\mathbf{O}_{2}, \\
\xi^{+} & =\xi+\varepsilon \Theta_{\eta^{+}}^{s}\left(\eta^{+}, \xi, \tau\right)+\frac{w_{\eta^{+}}}{\lambda} \ln \left|\frac{\kappa^{s} w}{\lambda}\right|+\mathbf{O}_{1}, \\
h^{+} & =h-\varepsilon \Theta_{\tau}^{s}\left(\eta^{+}, \xi, \tau\right)-\frac{w_{\tau}}{\lambda} \ln \left|\frac{\kappa^{s} w}{\lambda}\right|+\mathbf{O}_{2}, \\
\tau^{+} & =\tau+t^{+}+\frac{w_{h^{+}}}{\lambda} \ln \left|\frac{\kappa^{s} w}{\lambda}\right|+\mathbf{O}_{1}, \\
s^{+} & =s \cdot \operatorname{sign}(w) .
\end{aligned}
$$




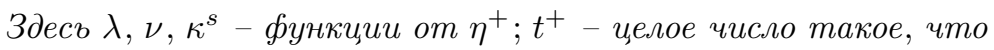

$$
\begin{gathered}
\left|\tau+t^{+}+\frac{w_{h^{+}}}{\lambda} \ln \right| \frac{\kappa^{s} w}{\lambda}||<c^{-1} ; \\
\mathbf{O}_{1}=O^{*}\left(\varepsilon^{7 / 8}\right) \ln ^{2} \varepsilon, \quad \mathbf{O}_{2}=O^{*}\left(\varepsilon^{5 / 4}\right) \ln ^{2} \varepsilon .
\end{gathered}
$$

Индекс s фиксирует сепаратрисную петлю, проходимую траекторией.

ЗАмЕчАнИЕ 6.2. 1) В [31] получены формулы для сепаратрисного отображения в более общей ситуации (без предположения $\mathbf{H}_{\mathbf{0}} \mathbf{4}$ о разделении переменных в невозмущенной системе).

2) Отображение за единичное время в переменных $(\eta, \xi, h, \tau, s)$ имеет вид

$$
(\eta, \xi, h, \tau, s) \mapsto(\eta, \xi, h, \tau+1, s)+(\varepsilon, 1, \varepsilon, 1,0) O^{*}\left(\varepsilon^{3 / 4}, h-E(\eta)\right) .
$$

3) При $t^{+}$, удовлетворяющем (6.9), сепаратрисное отображение задается равенствами

$$
\begin{gathered}
\eta=\mathscr{S}_{\xi}, \quad \xi^{+}=\mathscr{S}_{\eta^{+}}, \quad h=\mathscr{S}_{\tau}, \quad \tau^{+}=t^{+}+\mathscr{S}_{h^{+}}, \quad s^{+}=s \cdot \operatorname{sign}(w), \\
\mathscr{S}\left(\eta^{+}, \xi, h^{+}, \tau, s, t^{+}\right)=\left\langle\eta^{+}, \xi\right\rangle+h^{+} \tau+\varepsilon \Theta^{s}\left(\eta^{+}, \xi, \tau\right)+\frac{w}{\lambda} \ln \left|\frac{\kappa^{s} w}{\lambda e}\right|+O^{*}\left(\varepsilon^{9 / 8}\right) \ln ^{2} \varepsilon
\end{gathered}
$$

Отметим, что $\mathscr{S}$ зависит от параметра $t^{+}$лишь через последнее слагаемое.

$4)$ Если $n=0$, то переменные $(\eta, \xi)$ отсутствуют и получается обычное сепаратрисное отображение (3.3).

Функции $\lambda>0$ и $\kappa^{ \pm}>0$ определяются по невозмущенной системе, а $\Theta^{ \pm}$ оказываются некоторыми интегралами типа Пуанкаре-Мельникова.

Явные формулы для $\lambda, \kappa^{ \pm}$и $\Theta^{ \pm}$. Согласно $\mathbf{H}_{\mathbf{0}} \mathbf{2}$ собственные значения матрицы

$$
\Lambda(y)=\left(\begin{array}{cc}
-\left(H_{0}\right)_{u v}(y, 0,0), & -\left(H_{0}\right)_{u u}(y, 0,0) \\
\left(H_{0}\right)_{v v}(y, 0,0), & \left(H_{0}\right)_{v u}(y, 0,0)
\end{array}\right)
$$

вещественны. Их сумма равна $\operatorname{tr} \Lambda=0$. Функция $\lambda=\lambda(y)-$ положительное собственное значение $\Lambda$.

Пусть $\gamma^{ \pm}(y, \cdot): \mathbb{R} \rightarrow\left\{(v, u): H_{0}(y, v, u)=H_{0}(y, 0,0)\right\}$ - естественные параметризации сепаратрисных петель $\widehat{\gamma}^{ \pm}(y)$ :

$$
\dot{\gamma}^{ \pm}(y, t)=\left(-\left(H_{0}\right)_{u}\left(y, \gamma^{ \pm}(t)\right),\left(H_{0}\right)_{v}\left(y, \gamma^{ \pm}(t)\right)\right),
$$

и $a_{ \pm}=a_{ \pm}(y)$ - левые собственные векторы матрицы $\Lambda$ :

$$
a_{+} \Lambda=\lambda a_{+}, \quad a_{-} \Lambda=-\lambda a_{-}
$$

такие, что $(2 \times 2)$-матрица со строками $a_{+}$и $a_{-}$имеет единичный определитель.

ПРЕДЛОЖЕНИЕ 6.3 .

$$
\left(\kappa^{ \pm}\right)^{-1}(y)=\lim _{t \rightarrow+\infty}\left(\left\langle a_{+}(y), \gamma^{ \pm}(y,-t)\right\rangle\left\langle a_{-}(y), \gamma^{ \pm}(y, t)\right\rangle e^{2 \lambda t}\right) .
$$


Естественные параметризации на $\widehat{\gamma}^{ \pm}$определены с точностью до сдвига времени: $t \mapsto t+\varphi_{ \pm}(y)$. Назовем естественные параметризации согласованными, если они гладко зависят от $y$ и

$$
\lim _{t \rightarrow-\infty} \frac{\left\langle a_{+}(y), \gamma^{+}(y, t)\right\rangle}{\left\langle a_{+}(y), \gamma^{-}(y, t)\right\rangle}=-1 .
$$

Согласованные параметризации определены с точностью до одновременного сдвига: если $\gamma^{+}\left(y, t^{+}(y, t)\right), \gamma^{-}\left(y, t^{-}(y, t)\right)$ - другая пара согласованных параметризаций, то $t^{+}(y, t)=t^{-}(y, t)=t-t_{0}(y)$ с гладкой функцией $t_{0}$.

Любое решение невозмущенной системы, лежащее на $\widehat{\Gamma}^{ \pm}(y)$, имеет вид

$$
\begin{aligned}
(y, x, v, u)(t) & =\Gamma^{s}(y, \xi, \tau+t), \quad \xi \in \mathbb{T}^{n}, \quad \tau \in \mathbb{R}, \quad s \in\{+,-\} \\
\Gamma^{s}(y, \xi, \tau) & =\left(y, \xi+\nu(y) \tau, \gamma^{s}(y, \tau)\right) .
\end{aligned}
$$

Положим

$$
H_{*}^{s}(y, \xi, \tau, t)=H_{1}\left(\Gamma^{s}(y, \xi, t), t-\tau\right)-H_{1}(y, \xi+\nu t, 0,0, t-\tau) .
$$

Функции $H_{*}^{s}(y, \xi, \tau, t)$ экспоненциально стремятся к нулю при $t \rightarrow \pm \infty$.

ПреДЛОЖенИЕ 6.4. Предположим, что параметризации $\gamma^{ \pm}$согласованы. Тогда

$$
\Theta^{s}(y, \xi, \tau)=-\int_{-\infty}^{+\infty} H_{*}^{s}(y, \xi, \tau, t) d t .
$$

Функции $\Theta^{s}$ называются потенциалами расщепления. Они 1-периодичны по $\xi$ и $\tau$. В случае $y=$ const аналогичные функции были введены в [51] для $n=1$ и в [52] для произвольного $n$ и диофантова вектора частот $\nu\left(y^{0}\right)$.

Доказательства теоремы 6.1, а также предложений 6.3 и 6.4 имеются в [31].

\section{Глава 7. Размер стохастического слоя}

В этой главе мы используем сепаратрисное отображение Заславского для изучения стохастического слоя, возникающего в окрестности сепаратрис системы, близкой к интегрируемой. Описание основных результатов настоящей главы имеется в $§ 1$ главы 1.

Предположим, что отображение $T$ принадлежит гладкому семейству $T_{\varepsilon}, 0<$ $\varepsilon<\varepsilon_{0}$, гладких симплектоморфизмов, для которых выполнены условия (A)(C) из §8 главы 3. В частности, для них справедливы формулы (3.30).

Ниже нам иногда потребуется дополнительное условие симметрии.

(S) Отображение $\mathscr{S}_{\mathcal{E}}$ коммутирует с "центральной симметрией” $(x, y, s) \mapsto$ $(x, y,-s)$.

\section{§ 1. Теоремы о величине стохастического слоя}

Теоремы 7.2, 7.6 и 7.9, а также лемма 7.1, сформулированные в этом параграфе, будут доказаны в $\S 3$. 
Стохастическим слоем назовем область $\mathscr{S} L(\varepsilon) \subset D$, ограниченную непрерывными инвариантными кривыми $\gamma_{ \pm}$и $\gamma_{0}$ (см. рис. 1.1), ближайшими к гиперболической точке $\widehat{z}(\varepsilon)$. Ширина области $\mathscr{S} L(\varepsilon)$ будет измеряться в терминах функции $q p$, где, как обычно, $(q, p)=(q(\varepsilon), p(\varepsilon))$ - нормальные координаты, соответствующие гиперболической неподвижной точке $\sigma_{\varepsilon}$. Точнее, определим следующие величины:

$$
\underline{w}_{*}=\min _{(q, p) \in \gamma_{*}}|q p|, \quad \bar{w}_{*}=\max _{(q, p) \in \gamma_{*}}|q p|,
$$

где звездочка $*$ обозначает любой из символов,+- или 0 . Естественно считать, что ширина $w$ стохастического слоя изменяется внутри интервала $\left(\underline{w}_{\bullet}+\underline{w}_{0}, \bar{w}_{\bullet}+\right.$ $\left.\bar{w}_{0}\right)$, где

$$
\underline{w}_{\bullet}=\min \left\{\underline{w}_{+}, \underline{w}_{-}\right\}, \quad \bar{w}_{\bullet}=\max \left\{\bar{w}_{+}, \bar{w}_{-}\right\} .
$$

Ширина $d$ области $\mathscr{D}$ (см. рис. 1.1) определяется равенством

$$
d=\max _{\mathscr{D}} q p-\min _{\mathscr{D}} q p .
$$

Так как функция $q p$ не зависит от выбора нормальных координат, величины $\underline{w}_{*}$, $\bar{w}_{*}$ и $d$ корректно определены. Напомним, что $\mathscr{A}=\mathscr{A}(\varepsilon)-$ симплектическая площадь области $\mathscr{S} L(\varepsilon)$.

Лемма 7.1. Верны следующие соотношения:

1) $d \sim \delta, \mathscr{A}_{\mathscr{D}} \sim \lambda \delta$

2) если область $\mathscr{D}$ расположена на верхней (нижней) петле и $\nu_{+}(\xi)=$ a $\sin \left(2 \pi\left(\xi-\xi_{+}\right)\right)$(соответственно $\left.\nu_{-}(\xi)=a \sin \left(2 \pi\left(\xi-\xi_{-}\right)\right)\right)$, где а и $\xi_{ \pm}-$nостоянные, $\operatorname{mo} d=\delta(a+o(1))$ u $\mathscr{A}_{\mathscr{D}}=\lambda \delta(a / \pi+o(1))$ nри $\varepsilon \rightarrow 0$;

3) $\underline{A} \leqslant \mathscr{A} \leqslant \overline{\mathscr{A}}$, где

$$
\begin{aligned}
& \underline{\mathscr{A}}=2\left|\underline{w}_{0} \ln \underline{w}_{0}^{-1}+O\left(\underline{w}_{0}\right)\right|+\sum_{* \in\{+,-\}}\left|\underline{w}_{*} \ln \underline{w}_{*}^{-1}+O\left(\underline{w}_{*}\right)\right|, \\
& \overline{\mathscr{A}}=2\left|\bar{w}_{0} \ln \bar{w}_{0}^{-1}+O\left(\bar{w}_{0}\right)\right|+\sum_{* \in\{+,-\}}\left|\bar{w}_{*} \ln \bar{w}_{*}^{-1}+O\left(\bar{w}_{*}\right)\right| .
\end{aligned}
$$

Теорема 7.2. Предположим, что семейство отображений $T_{\varepsilon}$ удовлетворяет предположениям (A)-(C). Тогда при малых $\varepsilon>0$ верны следующие оценки:

$$
\begin{aligned}
c_{1} \delta_{\varepsilon} / \ln \mu_{\varepsilon} \leqslant \underline{w}_{ \pm} & \leqslant \bar{w}_{ \pm} \leqslant c_{2} \delta_{\varepsilon} / \ln \mu_{\varepsilon}, \\
\underline{w}_{0} & \leqslant \bar{w}_{0} \leqslant c_{2} \delta_{\varepsilon} / \ln \mu_{\varepsilon}
\end{aligned}
$$

с некоторыми постоянными $c_{1} u c_{2}$.

Более того, если семейство $T_{\varepsilon}$ удовлетворяет условию $(\mathbf{S})$, то

$$
\underline{w}_{0} \geqslant c_{1} \delta_{\varepsilon} / \ln \mu_{\varepsilon} .
$$

ЗАмЕчаниЕ 7.3. Согласно теореме 7.2, при выполнении условий $(\mathbf{A})-(\mathbf{C})$ ширина стохастического слоя меняется в интервале $\left[c_{1} \delta / \ln \mu, 2 c_{2} \delta / \ln \mu\right]$. Так как согласно лемме 7.1 ширина $d$ области $\mathscr{D}$, измеренная функцией $q p$, имеет тот же порядок малости, что и $\delta$, получаем оценку (1.1). 
ГиПотезА 7.4. Оценка (7.2) остается верной и без условия (S).

ЗАмечАниЕ 7.5. Согласно лемме 7.1 и теореме 7.2, для некоторых положительных постоянных $\widetilde{c}_{1}$ и $\widetilde{c}_{2}$

$$
\frac{\widetilde{c}_{1} \delta_{\varepsilon}}{\ln \mu_{\varepsilon}} \ln \frac{\ln \mu_{\varepsilon}}{\delta_{\varepsilon}} \leqslant \mathscr{A} \leqslant \frac{\widetilde{c}_{2} \delta_{\varepsilon}}{\ln \mu_{\varepsilon}} \ln \frac{\ln \mu_{\varepsilon}}{\delta_{\varepsilon}} .
$$

Теперь рассмотрим подробнее случай $\lim _{\varepsilon \rightarrow 0} \mu_{\varepsilon}=1$. Указанное равенство имеет место в случае, когда $T_{0}=\mathrm{id}$. Вычисление функций $\nu_{ \pm}$в указанной ситуации весьма сложно. Однако анализ сепаратрисного отображения оказывается более простым в силу следующих двух причин. Во-первых, появляется новый малый параметр: $\ln \mu$. Во-вторых, изучение явления экспоненциально малого расщепления сепаратрис в конкретных задачах (в стандартном отображении, в маятнике с быстро колеблющейся точкой подвеса и в некоторых других [53][59]) показывает, что в главном приближении 1-периодические функции $\nu_{ \pm}$содержат лишь младшие гармоники, т.е.

$$
\widehat{\nu}_{ \pm}(\xi, \varepsilon)=\nu_{ \pm}(\xi)+O(\varepsilon), \quad \nu_{ \pm}(\xi)=a_{ \pm} \sin \left(2 \pi\left(\xi-\xi_{ \pm}\right)\right),
$$

где $a_{ \pm}>0$ и $\xi_{ \pm} \in \mathbb{R} / \mathbb{Z}-$ постоянные. ${ }^{8}$

ТЕОРема 7.6. Предположим, что выполнены условия (A)-(C) и справедливы равенства

$$
\lim _{\varepsilon \rightarrow 0} \mu_{\varepsilon}=1, \quad \lim _{\varepsilon \rightarrow 0} \delta_{\varepsilon} / \ln ^{2} \mu_{\varepsilon}=0 .
$$

Тогда

$$
\bar{w}_{*}-\underline{w}_{*}=O\left(\delta_{\varepsilon}\right), \quad * \in\{+,-\} .
$$

Более того, если выполняется условие $(\mathbf{S})$, то $\bar{w}_{0}-\underline{w}_{0}=O(\delta)$.

СЛЕДСТВИЕ 7.7. Если все условия (включая и условие (S)) теоремы 7.6 выполнены, то, согласно теореме $7.2, \bar{w}_{*}, \underline{w}_{*} \sim O(\delta / \ln \mu)$, , следовательно, справедливы равенства $\bar{w}_{*} / \underline{w}_{*}=1+O(1 / \ln \mu), * \in\{+,-, 0\}$.

Ниже нам потребуются следующие определения. Пусть $C_{a, \omega, \lambda}$ - следующее симплектическое отображение циллиндра $(x \bmod 1, y)$ на себя:

$$
C_{a, \omega, \lambda}(x, y)=\left(x^{+}, y^{+}\right)=\left(x+\omega+y^{+}, y+\lambda^{-1} a \sin (2 \pi x)\right) .
$$

Отображение $C_{1,0, \varepsilon^{-1}}$ совпадает со стандартным (см. 11 введения).

Назовем замкнутую кривую на цилиндре топологически горизонтальной (ТГ-кривой), если она гомотопна окружности $\{y=0\}^{9}$. Замкнутую кривую $\gamma$, инвариантную относительно симплектического отображения $Q$ цилиндра в себя, назовем жесткой, если любое близкое к $Q$ гладкое симплектическое отображение имеет инвариантную кривую, близкую к $\gamma$. Определим три функции

$$
\bar{m}(a, \omega), \quad \underline{m}(a, \omega), \quad \bar{m}_{0}\left(a^{\prime}, a^{\prime \prime}, \omega^{\prime}, \omega^{\prime \prime}\right)
$$

\footnotetext{
${ }^{8}$ Есть большие основания ожидать, что равенства (7.3) выполняются для систем общего положения с экспоненциально малым расщеплением сепаратрис.

${ }^{9}$ Слово “горизонтальный" здесь выбрано по той причине, что цилиндр можно представлять себе вложенным в пространство $\mathbb{R}^{3}$ так, что его образующие - вертикальные прямые. Тогда окружность $\{y=0\}$ лежит в горизонтальной плоскости.
} 
следующим образом:

$$
\begin{aligned}
\bar{m}=\inf \left\{\lambda_{0}>0:\right. & \text { для любого } \lambda \geqslant \lambda_{0} \text { отображение } C_{a, \omega, \lambda} \\
& \text { имеет инвариантную жесткую ТГ-кривую }\}, \\
\underline{m}=\sup \left\{\lambda_{0}>0:\right. & \text { для любого } 0<\lambda<\lambda_{0} \text { отображение } C_{a, \omega, \lambda} \\
& \text { не имеет инвариантной ТГ-кривой }\}, \\
\bar{m}_{0}=\inf \left\{\lambda_{0}>0:\right. & \text { для любого } \lambda \geqslant \lambda_{0} \text { отображение } \\
& C_{a^{\prime}, \omega^{\prime}, \lambda} \circ C_{a^{\prime \prime}, \omega^{\prime \prime}, \lambda} \text { имеет инвариантную } \\
& \text { жесткую ТГ-кривую }\} .
\end{aligned}
$$

Опишем простейшие свойства этих функций. Начнем с $\underline{m}$ и $\bar{m}$. Так как $\omega$ легко исключается из $C_{a, \omega, \lambda}$ с помощью замены переменной $y$, функции $\underline{m}$ и $\bar{m}$ не зависят от $\omega$. Более того, очевидно,

$$
\underline{m}=\underline{c} a, \quad \bar{m}=\bar{c} a
$$

для некоторых постоянных $0<\underline{c} \leqslant \bar{c}$.

Значение постоянной $\underline{c}$ вычислялось в ряде работ (см., например, [60], [3], [61], [4], [62], [5]). Наиболее точные оценки получены численно [3], [61], [5]. В наших обозначениях результат следующий:

$$
\underline{c}=2 \pi / 0.971635 \ldots
$$

Более того, хорошо известна следующая гипотеза.

ГиПоТЕЗА 7.8. Постоянные $\underline{c}$ u $\bar{c}$ совпадают.

Гипотеза 7.8 подтверждается численными экспериментами.

Функция $\bar{m}_{0}$ также зависит от своих аргументов специальным образом. Несложно проверить следующие утверждения.

1) Выполняются неравенства $0<\bar{m}_{0}<\infty$.

2) Для любых а $и \omega$ имеем $\bar{m}_{0}(a, a, \omega, \omega)=\bar{c} a$.

3) Функиия $\bar{m}_{0}$ может быть представлена в виде

$$
\begin{gathered}
\bar{m}_{0}\left(a^{\prime}, a^{\prime \prime}, \omega^{\prime}, \omega^{\prime \prime}\right)=a \bar{f}\left(\eta, \omega^{\prime}-\omega^{\prime \prime}\right), \\
a^{\prime}=a \cos (2 \pi \eta), \quad a^{\prime \prime}=a \sin (2 \pi \eta),
\end{gathered}
$$

где функиия $\bar{f}$ 1-периодична по обоим аргументам и удовлетворяет тождествам

$$
\bar{f}(\eta, \omega)=\bar{f}(-\eta, \omega)=\bar{f}(1 / 2-\eta, \omega)=\bar{f}(1 / 4-\eta,-\omega), \quad \eta, \omega \in \mathbb{R} / \mathbb{Z} .
$$

ТЕОРема 7.9. Предположим, что выполнены условия (A)-(C),

$$
\lim _{\varepsilon \rightarrow 0} \mu_{\varepsilon}=1, \quad \lim _{\varepsilon \rightarrow 0} \delta_{\varepsilon} / \ln ^{2} \mu_{\varepsilon}=0
$$

и верны равенства (7.3). Тогда справедливы следующие ощенки: 
а) величины $\underline{w}_{ \pm} u \bar{w}_{ \pm}$удовлетворяют неравенствам

$$
a_{ \pm} \underline{c}(1+o(1)) \leqslant \ln \mu \cdot \underline{w}_{ \pm} / \delta \leqslant \ln \mu \cdot \bar{w}_{ \pm} / \delta \leqslant a_{ \pm} \bar{c}(1+o(1)) ;
$$

б) в случае $\alpha_{+} \neq \alpha_{-}$

$$
\ln \mu \cdot \underline{w}_{0} / \delta \leqslant \ln \mu \cdot \bar{w}_{0} / \delta \leqslant \sup _{\omega^{\prime}, \omega^{\prime \prime}} \bar{m}_{0}\left(a_{+}, a_{-}, \omega^{\prime}, \omega^{\prime \prime}\right)(1+o(1)) ;
$$

в) если $\alpha_{+}=\alpha_{-}$, mo

$$
\ln \mu \cdot \underline{w}_{0} / \delta \leqslant \ln \mu \cdot \bar{w}_{0} / \delta \leqslant \bar{m}_{0}\left(a_{+}, a_{-}, 2 \xi_{+}, 2 \xi_{-}\right)(1+o(1)) ;
$$

г) если условие $(\mathbf{S})$ выполнено, то $\underline{w}_{0} u \bar{w}_{0}$ могут быть оценены аналогично величинам $\underline{w}_{ \pm} u \bar{w}_{ \pm}$:

$$
a_{+} \underline{c}(1+o(1)) \leqslant \ln \mu \cdot \underline{w}_{0} / \delta \leqslant \ln \mu \cdot \bar{w}_{0} / \delta \leqslant a_{+} \bar{c}(1+o(1)) .
$$

СлЕДСТвИЕ 7.10. Если все условия (включая (S)) теоремы 7.6 выполнены, то верхняя $\bar{w}$ и нижняя $\underline{w}$ оценки ширины стохастического слоя

$$
\bar{w}=\max \left\{\bar{w}_{+}+\bar{w}_{0}, \bar{w}_{-}+\bar{w}_{0}\right\}, \quad \underline{w}=\min \left\{\underline{w}_{+}+\underline{w}_{0}, \underline{w}_{-}+\underline{w}_{0}\right\}
$$

удовлетворяют неравенствам

$$
2 a_{+} \delta \underline{c}(1+o(1)) \leqslant \underline{w} \ln \mu \leqslant \bar{w} \ln \mu \leqslant 2 a_{+} \delta \bar{c}(1+o(1)) .
$$

ЗАмЕчАниЕ 7.11. Если верна гипотеза 7.8, то из соотношений (7.4) и (7.5) следуют равенства

$$
\lim _{\varepsilon \rightarrow 0}\left(\ln \mu \cdot w_{*} / \delta\right)=a_{+} \underline{c}=a_{+} \bar{c}, \quad * \in\{+,-, 0\} .
$$

Из оценок (7.6), (7.7) вытекает формула (1.3). Действительно, величины $w(\varepsilon)$ и $d(\varepsilon)$, измеренные в терминах $q p$, имеют следующий вид:

$$
w(\varepsilon) \approx \underline{w} \approx \bar{w}, \quad d(\varepsilon)=\delta a_{+}=\delta a_{-} .
$$

Последние два равенства следуют из леммы 7.1. С помощью леммы 7.1 легко получаются также оценки, содержащиеся в следующем замечании.

ЗАмЕчАниЕ 7.12. Если все условия теоремы 7.6 выполнены и верна гипотеза 7.8, то площадь стохастического слоя удовлетворяет соотношению

$$
\mathscr{A}=\frac{4 \underline{c} a_{+} \delta_{\varepsilon}}{\ln \mu_{\varepsilon}} \ln \delta^{-1}(\varepsilon)(1+o(1)) .
$$

Площадь области $\mathscr{D}$, ограниченной сепаратрисами (см. рис. 1.1), равна

$$
\mathscr{A}_{\mathscr{D}}=\frac{a_{+} \delta_{\varepsilon} \ln \mu_{\varepsilon}}{\pi}(1+o(1)) .
$$

Оценка (1.5) следует из равенств (7.8), (7.9).

В случае $\alpha_{+} \neq \alpha_{-}$величины $\ln \mu \cdot \underline{w}_{0} / \delta$ и $\ln \mu \cdot \bar{w}_{0} / \delta$, по-видимому, не имеют предела при $\varepsilon \rightarrow 0$, а осциллируют между двумя положительными постоянными. 


\section{§ 2. Стохастический слой в маятнике с колеблющейся точкой подвеса}

Рассмотрим еще раз отображение Пуанкаре для маятника с периодически колеблющейся точкой подвеса. Величины $\alpha_{ \pm}$и $\nu_{ \pm}$вычислены в $\S 7$ главы 3. Рассматриваемое сепаратрисное отображение удовлетворяет условию симметрии $(\mathbf{S})$, так как инволюция $(\widehat{u}, \widehat{v}) \mapsto(-\widehat{u},-\widehat{v})$ сохраняет гамильтониан $(3.25)$ (см. §7). Согласно теореме 7.2 ширина стохастического слоя в окрестности сепаратрисной восьмерки с центром в точке $\widehat{u}=\widehat{v}=0$ имеет порядок $\varepsilon$ при условии, что $\theta, \Omega, \omega \sim 1$ и $\theta$ не постоянна. При тех же предположениях площадь $\mathscr{A}$ стохастического слоя имеет порядок $\varepsilon \ln \varepsilon^{-1}$.

Равенство (3.26) справедливо в случае отношения частот $\omega / \Omega \sim 1$. В случае $\omega / \Omega \sim \varepsilon^{-1}$ стандартная теория Пуанкаре-Мельникова перестает работать и приходится применять другие методы, годящиеся для исследования экспоненциально малых эффектов.

Первые нетривиальные строгие результаты, количественно описывающие экспоненциально малое расщепление сепаратрис, получены в работе [54] (см. также [57]). К настоящему времени имеется по крайней мере две параллельные теории, эффективно работающие при анализе широкого круга задач такого типа. Первая из них создана В. Ф. Лазуткиным с соавторами [54]-[56], [63]. Вторая была развита в [58], [59].

Рассмотрим систему с гамильтонианом (3.25) (§7 главы 3) при следующих предположениях ${ }^{10}$ :

$$
\Omega=1, \quad \omega=1 / \varepsilon, \quad \theta(s)=2 \varepsilon^{-1} B \cos s .
$$

Старший мультипликатор гиперболической неподвижной точки $\widehat{u}=\widehat{v}=0$ соответствующего отображения Пуанкаре имеет вид $\mu=e^{2 \pi \varepsilon+O\left(\varepsilon^{2}\right)}$.

Сепаратрисное отображение удовлетворяет условию (S). Следуя [59], можно показать, что существует симплектическая замена координат $\widehat{u}, \widehat{v} \mapsto \widetilde{u}, \widetilde{v}$ которая

i) близка к тождественной,

ii) периодична по времени,

iii) вещественно-аналитична в комплексной окрестности сепаратрис $\Gamma^{ \pm}$системы с гамильтонианом $H_{0}=\widehat{v}^{2} / 2+\cos \widehat{u}$,

iv) такая, что новая функция Гамильтона принимает вид:

$$
\widetilde{H}(\widetilde{u}, \widetilde{v}, t, \varepsilon)=H_{0}(\widetilde{u}, \widetilde{v})+\varepsilon \widetilde{H}_{1}(\widetilde{u}, \widetilde{v}, \varepsilon)+\exp (-\widetilde{c} / \varepsilon) \widetilde{H}_{2}(\widetilde{u}, \widetilde{v}, t, \varepsilon) .
$$

Здесь $H_{0}=\widetilde{v}^{2} / 2+\cos \widetilde{u}$, постоянная $\widetilde{c} \in[0, \pi / 2)$ произвольна, функции $\widetilde{H}_{1}, \widetilde{H}_{2}$ вещественно-аналитичны по $\widetilde{u}, \widetilde{v}$ в окрестности $\Gamma^{ \pm}$, гладкие по $\varepsilon>0$ и функция $\widetilde{H}_{2} 2 \pi$-периодична по $t$.

При любом положительном $\widetilde{c} \in(\pi / 4, \pi / 2)$ обычные методы теории Пуанкаре-Мельникова, примененные к этой системе, дают правильную асимптотику расщепления сепаратрис. В частности, удается получить:

$$
\nu_{ \pm}(t)=-2 \pi \varepsilon^{-2} e^{-\pi \varepsilon^{-1} / 2} B f\left(B^{2}\right) \sin (2 \pi t),
$$

\footnotetext{
${ }^{10}$ Равенства (7.10) означают, что амплитуда колебаний точки подвеса имеет порядок $\varepsilon^{-2}$.
} 
где $f$ - целая вещественно-аналитическая функция, $f(0)=2$.

Согласно теореме 7.9 ширина стохастического слоя возле сепаратрис $\Gamma^{ \pm}$удовлетворяет оценке (7.6) с

$$
a_{+} \delta=2 \pi \varepsilon^{-2} e^{-\pi \varepsilon^{-1} / 2} B f\left(B^{2}\right), \quad \ln \mu=2 \pi \varepsilon .
$$

Площадь стохастического слоя оценивается следующим образом:

$$
\frac{2 \pi \underline{c}}{\varepsilon^{4}} e^{-\pi \varepsilon^{-1} / 2} B f\left(B^{2}\right)(1+o(1)) \leqslant \mathscr{A} \leqslant \frac{2 \pi \bar{c}}{\varepsilon^{4}} e^{-\pi \varepsilon^{-1} / 2} B f\left(B^{2}\right)(1+o(1)) .
$$

(Напомним, что гипотеза 7.8 утверждает, что $\underline{c}=\bar{c}$.)

\section{§ 3. Теория КАМ и теорема Биркгофа}

В этом параграфе мы доказываем теоремы 7.2, 7.6 и лемму 7.1.

ДокАЗАТЕЛЬСТво лЕммы 7.1. Согласно (3.30) неустойчивая сепаратриса задается уравнением $y=0$, а устойчивая - уравнением $y_{+}=y_{+}(x, y)=0$. Последнее равенство приводится к виду

$$
y=-\ln \mu\left(\widehat{\nu}_{s}(x, \varepsilon)+O(\delta)\right)=-\ln \mu\left(\nu_{s}(x)+o(1)\right) \quad \text { при } \quad \varepsilon \rightarrow 0 .
$$

Таким образом, гомоклинические точки соответствуют нулям функций $\nu_{ \pm}(x)$. Область $\mathscr{D}$ определяется двумя соседними нулями $x_{1}, x_{2}$ функции $\nu_{s}$.

Пусть $x_{1}$ и $x_{2}$ определяют область $\mathscr{D}$. Функция $\nu_{s}$ сохраняет знак на интервале $\left(x_{1}, x_{2}\right)$. Из (7.1) и (3.29) получаем формулу для ширины области $\mathscr{D}$ :

$$
d=\delta\left(\max _{x_{1} \leqslant x \leqslant x_{2}} \nu_{s}(x)+o(1)\right) \quad \text { при } \quad \varepsilon \rightarrow 0 .
$$

Площадь $\mathscr{A}_{\mathscr{D}}=\mathscr{A}_{\mathscr{D}}^{s}, s= \pm$, определяется как абсолютная величина интеграла $\int_{\mathscr{D}} d p \wedge d q=\delta \int_{\mathscr{D}} d y \wedge d x$. Следовательно, имеем:

$$
\mathscr{A}_{\mathscr{D}}^{s}=\delta \ln \mu\left|\int_{x_{1}}^{x_{2}} \nu_{s}(x) d x+o(1)\right| .
$$

Из равенств (7.11), (7.12) вытекают утверждения 1), 2) леммы 7.1.

Докажем утверждение 3 ). В нормальных координатах $q, p$ стохастический слой занимает область $\mathscr{L}$ такую, что $\mathscr{D} \subset \mathscr{L} \subset \overline{\mathscr{D}}$, где

$$
\begin{aligned}
& \underline{\mathscr{D}}=\left\{(q, p): q^{2}+p^{2}<\underline{r}^{2}, \quad|q p|<\left\{\begin{array}{ll}
\underline{w}_{+}, & \text {если } q>0 \text { и } p>0, \\
\underline{w}_{-}, & \text {если } q<0 \text { и } p<0, \\
\underline{w}_{0}, & \text { если } q p<0
\end{array}\right\},\right. \\
& \overline{\mathscr{D}}=\left\{(q, p): q^{2}+p^{2}<\bar{r}^{2}, \quad|q p|<\left\{\begin{array}{ll}
\bar{w}_{+}, & \text {если } q>0 \text { и } p>0, \\
\bar{w}_{-}, & \text {если } q<0 \text { и } p<0, \\
\bar{w}_{0}, & \text { если } q p<0
\end{array}\right\}\right.
\end{aligned}
$$

и $0<\underline{r} \leqslant \bar{r}-$ постоянные. Требуемая оценка для $\mathscr{A}$ вытекает из неравенств

$$
\operatorname{area}(\underline{\mathscr{D}}) \geqslant \underline{\mathscr{A}}, \quad \operatorname{area}(\overline{\mathscr{D}}) \leqslant \overline{\mathscr{A}} .
$$

Лемма 7.1 доказана. 
С учетом равенств (3.29) теоремы 7.2 и 7.6 вытекают из следующего утверждения.

ТЕОРема 7.13. Предположим, что $\varepsilon>0$ достаточно мал, $1<\mu<\mu_{0} u$

$$
\lim _{\varepsilon \rightarrow 0} \delta(\varepsilon) / \ln ^{2} \mu(\varepsilon)=0 .
$$

Тогда существуют две постоянные $0<y_{\min }<y_{\max }$ (зависящие только от функций $\left.\nu_{ \pm}\right)$такие, что при малых в справедливы следующие утверждения.

1) На инвариантных кривых $\gamma_{ \pm}, \gamma_{0}$ выполняется неравенство $|y|<y_{\max }$.

2) Кривые $\gamma_{+}$и $\gamma_{-}$не имеют общих точек с областью $|y| \leqslant y_{\min }$.

3) Выполняется оценка $y_{\max }-y_{\min }<2 y_{\min } \mu^{2} \ln \mu$.

4) Если условие (S) выполнено, то кривая $\gamma_{0}$ также не имеет общих точек с областью $|y| \leqslant y_{\min }$.

ДОКАЗАТЕЛЬСТВО ТЕОРЕМЫ 7.13. 1. Кривые $\gamma_{+}, \gamma_{-}$и $\gamma_{0}$ расположены в областях $y>0, s>0 ; y>0, s<0$; и $y<0$ соответственно. Анализ удобнее проводить в новых новых переменных $u, u_{+}$, определенных формулами

$$
y=y_{0}(1+u \ln \mu), \quad y_{+}=y_{0}\left(1+u_{+} \ln \mu\right), \quad y_{0}=\text { const. }
$$

Сепаратрисное отображение (3.30) переписывается в виде

$$
\begin{aligned}
& x_{+}=x+\omega_{s}+\frac{1}{\ln \mu} \ln \left|1+u_{+} \ln \mu+y_{0}^{-1} \delta \ln \mu \cdot O\left(1+y_{0} / \ln \mu\right)^{2}\right|, \\
& u_{+}=u+I_{0}^{-1} \widehat{\nu}_{s}(x, \varepsilon)+y_{0}^{-1} \delta \cdot O\left(1+y_{0} / \ln \mu\right)^{2}, \\
& s_{+}=s \cdot \operatorname{sign}\left(y_{0}\right) \cdot \operatorname{sign}\left(1+u_{+} \ln \mu+y_{0}^{-1} \delta \ln \mu \cdot O\left(1+y_{0} / \ln \mu\right)^{2}\right),
\end{aligned}
$$

где величины $\omega_{s}$ имеют вид

$$
\omega_{s}=\frac{1}{\ln \mu} \ln \frac{\left|y_{0}\right| \delta}{\alpha_{s}^{2} \ln \mu} .
$$

Основными параметрами в системе (7.13) являются $\delta$ и $y_{0}^{-1}$. Первый из них мал. Параметр $y_{0}$ ("невозмущенное действие") отвечает за тип динамики (регулярный или хаотический). Положив формально $y_{0}^{-1}=\delta \ln \mu \cdot O(1+y / \ln \mu)=0$, получаем интегрируемое отображение

$$
\begin{aligned}
& x_{+}=x+\omega_{s}+\frac{1}{\ln \mu} \ln \left|1+u_{+} \ln \mu\right|, \\
& u_{+}=u, \\
& s_{+}=s \cdot \operatorname{sign}\left(I_{0}\right) .
\end{aligned}
$$

Это отображение невырождено, так как $\partial x_{+}(x, u) / \partial u=(1+u \ln \mu)^{-1} \neq 0$.

Предположим, что справедливы следующие неравенства:

$$
\left|y_{0}^{-1}\right| \leqslant \widehat{c}_{1}^{-1} \ll 1, \quad \delta \leqslant \widehat{c}_{2}^{-1} \ll \frac{\widehat{c}_{1}}{\ln \mu \cdot\left(1+\widehat{c}_{1} / \ln \mu\right)^{2}} .
$$

Тогда согласно теории КАМ сепаратрисное отображение (7.13) имеет инвариантную кривую $u=u(x)=u_{0}+O\left(y_{0}^{-1}\right)$, где значение $u_{0}$ таково, что "невозмущенная" частота $\omega_{s}+(\ln \mu)^{-1} \ln |1+u \ln \mu|$ диофантова. (Очевидно, можно 
взять $\left|u_{0}\right|<1 /(2 \ln \mu)$.) Напомним, что при $y_{0}>0$ инвариантная кривая гомотопна $\gamma_{s}$, а при $y_{0}<0$ инвариантная кривая гомотопна $\gamma_{0}$. Утверждение 1$)$ теоремы 7.13 доказано, так как можно положить $y_{\max }=\max _{x}\left|y_{0}\right|(1+u(x) \ln \mu)$.

2. Мы докажем утверждение 2) лишь для кривой $\gamma_{+}$. Случай кривой $\gamma_{-}$ аналогичен. Во-первых, заметим, что при малых значениях $\varepsilon>0$ отображение (7.13) закручивающее. Это означает, что $\partial x_{+}(x, y) / \partial y>0$. Следуя идее Лазуткина [10], мы используем следующий результат Биркгофа [64] (см. также [65]).

ЛЕмма 7.14. Пусть $\gamma$ - непрерывная инвариантная кривая закручивающего симплектического отображения. Если $\gamma$ гомотопна кривой $\{y=$ const $\}$, то $\gamma$ является графиком липшищевой функции $y=r(x)$.

Липшицева константа функции $r(x)$ может быть оценена следующим образом. Пусть $Q=\partial\left(x_{+}, y_{+}\right) / \partial(x, y)$ - матрица Якоби отображения $T_{\varepsilon}$. Так как $T_{\varepsilon}$ - закручивающее отображение, существуют постоянные $\eta_{1}, \eta_{2}>0$ такие, что для любой точки $(x, y)$ на кривой выполняются неравенства

$$
(1,0) Q\left(\begin{array}{c}
-1 \\
\eta_{1}
\end{array}\right)>0, \quad(1,0) Q^{-1}\left(\begin{array}{c}
1 \\
\eta_{2}
\end{array}\right)<0 .
$$

Следующая лемма фактически содержится в [65].

Лемма 7.15. Для любых двух точек $x_{1}, x_{2}$ таких, что $0 \leqslant x_{2}-x_{1}<1 / 2$, функция $r(x)$ удовлетворяет неравенствам

$$
-\eta_{1}\left(x_{2}-x_{1}\right) \leqslant r\left(x_{2}\right)-r\left(x_{1}\right) \leqslant \eta_{2}\left(x_{2}-x_{1}\right) .
$$

Лемма 7.15 является следствием леммы 7.14. Действительно, предположив, что по крайней мере одно из неравенств (7.14) не выполняется для некоторой пары $x_{1}<x_{2}$, где $x_{1}$ и $x_{2}$ близки, получаем, что для образов $\left(\widetilde{x}_{1}, r\left(\widetilde{x}_{1}\right)\right)$, $\left(\widetilde{x}_{2}, r\left(\widetilde{x}_{2}\right)\right)$ точек $\left(x_{1}, r\left(x_{1}\right)\right),\left(x_{2}, r\left(x_{2}\right)\right)$ при отображении $T_{\varepsilon}$ (или $\left.T_{\varepsilon}^{-1}\right)$ справедливо неравенство $\widetilde{x}_{2}<\widetilde{x}_{1}$, что противоречит лемме 7.14 .

Пусть кривая $\gamma_{+}$задается параметризацией $(x, y)=\left(x, r_{+}(x)\right)$. Ниже мы покажем, что

$$
r_{\max }=\max _{x} r_{+}(x)
$$

оценивается снизу положительной постоянной (предложение 7.16), а затем что $r_{+}(x)>r_{\max } e^{-2 \ln \mu}$ (предложение 7.17 ).

Из предложений 7.16, 7.17 следует утверждение 2) теоремы 7.13 для кривой $\gamma_{+}$. Утверждение 3) также вытекает из предложения 7.17. Действительно, имеем неравенство $y_{\max }<y_{\min } \mu^{2}$. Пользуясь простым соотношением

$$
\mu^{2}<1+2 \mu^{2} \ln \mu, \quad \mu>1
$$

получаем требуемую оценку.

Функция $r_{+}$- положительная, 1-периодичная и липшицева. Оценим липшицеву константу для малых значений параметра $\varepsilon$ с помощью леммы 7.15. 
Сначала положим $\varepsilon=0$ (при этом автоматически получим $\delta=0$ ). Матрица Якоби $Q$ принимает вид

$$
Q=\left(\begin{array}{cc}
1+x_{+}^{-1} \nu_{+}^{\prime}(x) & \left(x_{+} \ln \mu\right)^{-1} \\
\ln \mu \cdot \nu_{+}^{\prime}(x) & 1
\end{array}\right),
$$

где $x_{+}=r_{+}(x)+\ln \mu \cdot \nu_{+}^{\prime}(x)=r_{+}\left(x_{+}\right)$и $\nu_{+}^{\prime}=d \nu_{+} / d x$. В результате прямых вычислений получаем: $\eta_{2} \geqslant r_{\max } \ln \mu$. При малых ненулевых значениях $\delta$ оценка слегка слабее. Можно положить

$$
\eta_{2}=2 r_{\max } \ln \mu \text {. }
$$

Определим постоянную $\nu_{\min }^{\prime}$ следующим образом:

$$
\nu_{\min +}^{\prime}=\min _{x} d \nu_{+} / d x
$$

Так как согласно предположению (B) функция $\nu_{+}$периодична и непостоянна, имеем $\nu_{\min }^{\prime}<0$.

ПРЕДЛОЖЕНИЕ 7.16. Предположим, что параметр $\varepsilon>0$ достаточно мал. Тогда

$$
r_{\max } \geqslant-\nu_{\min +}^{\prime} / 7 \text {. }
$$

ДоКАЗАТЕЛЬСТВо ПРЕДЛОЖЕНИЯ 7.16. Сначала положим $\varepsilon=0$. Пусть отображение (7.13) преобразует точки $\left(x_{j}, r_{+}\left(x_{j}\right)\right), j=1,2$, в $\left(\widetilde{x}_{j}, r_{+}\left(\widetilde{x}_{j}\right)\right)$.

Предположим, что точки $x_{1}, x_{2}$ близки и $x_{1}<x_{2}$. Тогда точки $\widetilde{x}_{1}, \widetilde{x}_{2}$ также близки друг к другу и $\widetilde{x}_{1}<\widetilde{x}_{2}$. Используя равенства $(7.13)$, имеем:

$$
\Delta \widetilde{x}=\Delta x+\frac{1}{\ln \mu} \ln \left(1+\frac{\Delta r_{+}+\ln \mu \cdot \Delta \nu_{+}}{r_{+}\left(x_{1}\right)+\ln \mu \cdot \nu_{+}\left(x_{1}\right)}\right)
$$

где

$$
\begin{gathered}
\Delta \widetilde{x}=\widetilde{x}_{2}-\widetilde{x}_{1}, \quad \Delta x=x_{2}-x_{1}>0, \\
\Delta r_{+}=r_{+}\left(x_{2}\right)-r_{+}\left(x_{1}\right), \quad \Delta \nu_{+}=\nu_{+}\left(x_{2}\right)-\nu_{+}\left(x_{1}\right) .
\end{gathered}
$$

Возьмем $x_{1}$ таким, что $\nu_{+}^{\prime}\left(x_{1}\right)=\nu_{\min }^{\prime} \cdot$ Для малых положительных $\Delta x$ имеем оценки:

$$
\Delta r_{+} \leqslant \eta_{2} \Delta x \leqslant 2 r_{\max } \ln \mu \cdot \Delta x, \quad \Delta \nu_{+} \leqslant \nu_{\min +}^{\prime} \Delta x / 2<0 .
$$

Здесь мы использовали соотношения (7.14), (7.15). Теперь предположим, что $r_{\max }<-\nu_{\min +}^{\prime} / 6$. Тогда из равенства (7.17) следует оценка

$$
\begin{aligned}
\Delta \widetilde{x} & <\Delta x+\frac{1}{\ln \mu} \ln \left(1+\frac{2 r_{\max }+\nu_{\min +}^{\prime} / 2}{r_{\max }} \Delta x \ln \mu\right) \\
& <\Delta x+\frac{\left(2 r_{\max }+\nu_{\min +}^{\prime} / 2\right) \Delta x}{r_{\max }}<\frac{\left(3 r_{\max }+\nu_{\min +}^{\prime} / 2\right)}{\Delta x / r_{\max }}<0 .
\end{aligned}
$$

Это противоречит неравенству $\Delta \widetilde{x}>0$. При малых значениях $\varepsilon$ оценки сохраняются (возможно, со слегка измененными постоянными). Поэтому мы взяли в (7.16) $\nu_{\min +}^{\prime} / 7$ вместо $\nu_{\min +}^{\prime} / 6$. Предложение 7.16 доказано. 
ПреДЛОЖЕНИЕ 7.17. Для любого $x \in \mathbb{R} / \mathbb{Z}$ имеем $r_{\max } / r(x)<\mu^{2}$.

ДОКАЗАТЕЛЬСТВО ПРЕДЛОЖЕНИЯ 7.17. Положим $\varepsilon=0$ и доПустим, что существует точка $\widehat{x} \in \mathbb{R} / \mathbb{Z}$ такая, что $r_{\max } \geqslant r(\widehat{x}) \mu$. Можно считать, что $0<$ $\widehat{x}-x_{0}<1$, где точка $x_{0}$ удовлетворяет равенству $r\left(x_{0}\right)=r_{\max }$. Возьмем пару точек $\theta_{0}, \widehat{\theta} \in \mathbb{R} / \mathbb{Z}$ такую, что $\left.(7.13)\right|_{\varepsilon=0}$ отображает точки $\left(\theta_{0}, r\left(\theta_{0}\right)\right),(r(\widehat{\theta}), \widehat{\theta})$ соответственно в $\left(r\left(x_{0}\right), x_{0}\right),(r(\widehat{x}), \widehat{x})$.

Покажем, что $\widehat{\theta}-\theta_{0}>1$ в том смысле, что образ отрезка

$$
\left\{(y, x): y=r(x), \widehat{\theta} \leqslant x \leqslant \theta_{0}\right\}
$$

накрывает всю окружность при естественной проекции на $\mathbb{R} / \mathbb{Z}:(y, x) \mapsto x$. Доказав этот факт, мы получим противоречие с леммой 7.14, результатом которого и будет доказательство предложения 7.17.

Величина $\widehat{\theta}-\theta_{0}$ оценивается с помощью равенств $\left.(7.13)\right|_{\varepsilon=0}$ :

$$
\widehat{x}-x_{0}=\widehat{\theta}-\theta_{0}+\frac{1}{\ln \mu} \ln \frac{r(\widehat{x})}{r\left(x_{0}\right)} .
$$

Следовательно, имеем:

$$
\widehat{\theta}-\theta_{0}=\widehat{x}-x_{0}-\frac{1}{\ln \mu} \frac{r(\widehat{x})}{r_{\max }}>1 .
$$

В случае малых ненулевых $\varepsilon$ оценки несколько слабее. Из-за этого в предложении 7.17 мы предполагаем, что $r_{\max } / r(x)<\mu^{2}$. Предложение 7.17 доказано.

Условие (S) позволяет аналогично доказать утверждение 4) теоремы 7.13.

\section{Глава 8. Островки устойчивости в сепаратрисных лунках}

В этой главе мы докажем теоремы 1.1 и 1.3 о существование островков устойчивости в сепаратрисных лунках для симплектических отображений близких к интегрируемым. Основные результаты и обозначения даны в 2 введения. При доказательстве основным инструментом является сепаратрисное отображение Заславского (глава 3).

\section{§ 1. Следствия из обратимости}

Начнем с теоремы 1.3. Рассмотрим семейство двумерных $I_{\varepsilon}$-обратимых симплектических отображений $T_{\varepsilon}$, близких к интегрируемому.

Лемма 8.1. Пусть гиперболический поворот $\mathscr{T}(q, p)=(q \mathscr{M}(p q), p / \mathscr{M}(p q))$ обратим относительно инволющии $\mathscr{I}$, сохраняющей I и III четверти $\mathbb{R}_{q, p}^{2}$. Тогда существуют нормальные координаты $(\widetilde{p}, \widetilde{q})$, в которых

$$
\mathscr{I}(\widetilde{q}, \widetilde{p})=(\widetilde{p}(1+O(\widetilde{q} \widetilde{p})), \widetilde{q}(1+O(\widetilde{q} \widetilde{p}))) .
$$

ДокАЗАтельство. Рассмотрим функцию $\Phi(p, q)=q p$. Сначала покажем, что

$$
\Phi \circ \mathscr{I}=\Phi+O\left(\Phi^{2}\right)
$$


Действительно, из определения обратимости имеем:

$$
\mathscr{I} \circ \mathscr{T}=\mathscr{T}^{-1} \circ \mathscr{I} .
$$

Поскольку $\Phi$ - первый интеграл $\mathscr{T}:$

$$
\Phi \circ \mathscr{I} \circ \mathscr{T}=\Phi \circ \mathscr{T}^{-1} \circ \mathscr{I}=\Phi \circ \mathscr{I} .
$$

Отсюда $\Phi \circ \mathscr{I}$ также первый интеграл $\mathscr{T}$. Из предложения $3.5 \Phi \circ \mathscr{I}=c_{0}+$ $c_{1} \Phi+O\left(\Phi^{2}\right)$. Более того, $c_{0}=0$, поскольку $\mathscr{I}(0)=0$. Далее имеем:

$$
\Phi=\Phi \circ \mathscr{I} \circ \mathscr{I}=c_{1} \Phi \circ \mathscr{I}+O(\Phi \circ \mathscr{I})^{2}=c_{1}^{2} \Phi+O\left(\Phi^{2}\right) .
$$

Следовательно, $c_{1}= \pm 1$. На самом деле, $c_{1}=1$, поскольку $\mathscr{I}$ сохраняет I и III четверти $\mathbb{R}_{q, p}^{2}$. Отсюда получаем (8.2).

Пусть $\mathscr{I}^{(q)}$ и $\mathscr{I}^{(p)}$ - первая и вторая компонента образа $\mathscr{I}$. Поскольку $\mathscr{I}$ меняет местами устойчивую и неустойчивую сепаратрисы, имеем:

$$
\mathscr{I}^{(q)}(q, 0)=0, \quad \mathscr{I}^{(p)}(0, p)=0 .
$$

Следовательно, мы можем положить

$$
\mathscr{I}^{(q)}(q, p)=p a(p, q), \quad \mathscr{I}^{(p)}(q, p)=q b(p, q),
$$

где $a$ и $b$ - гладкие функции. Используя (8.3) и (8.2), получаем:

$$
a(q \mu, p / \mu)=a(p, q)+O(q p), \quad b(q \mu, p / \mu)=b(p, q)+O(q p) .
$$

Следовательно,

$$
a(q, p)=a(0,0)+O(q p), \quad b(q, p)=b(0,0)+O(q p) .
$$

Согласно $(8.2)$ имеем $a(0,0) b(0,0)=1$. Поскольку инволюция $\mathscr{I}$ сохраняет I и III четверти, то $a(0,0)>0$. Полагая $p=\widetilde{p} \sqrt{a(0,0)}, q=\widetilde{q} / \sqrt{a(0,0)}$ (см. пункт 3 ) замечания 2.2$)$, получаем (8.1).

Пусть $T_{\varepsilon} I_{\varepsilon}$-обратимы, где $I_{\varepsilon}-$ семейство инволюций, сохраняющих петли. Тогда из леммы 8.1 и замечания 3.4 следует, что в окрестности гиперболической неподвижной точки $\sigma_{\varepsilon}$ существуют нормальные координаты $(q, p)=\left(q_{\varepsilon}, p_{\varepsilon}\right)$, в которых $I_{\varepsilon}$ имеет вид (8.1).

Ниже удобно заменить переменную $x$, определенную в $(3.13)$, на $x-\left(\ln \alpha_{ \pm}\right) / \lambda$. В новых координатах сепаратрисное отображение (3.1) имеет вид:

$$
\mathscr{S M}_{\varepsilon}\left(\begin{array}{l}
x \\
y \\
s
\end{array}\right)=\left(\begin{array}{c}
x^{+} \\
y^{+} \\
s^{+}
\end{array}\right)=\left(\begin{array}{c}
x+\frac{1+O(\varepsilon)}{\lambda}\left(\ln \frac{\varepsilon}{\alpha_{s}^{2} \lambda}+\ln \left|y^{+}\right|\right) \\
y+\lambda \nu_{s}\left(x-\left(\ln \alpha_{s}\right) / \lambda\right)+O(\varepsilon) \\
s \cdot \operatorname{sign}\left(y^{+}\right)
\end{array}\right) .
$$

Также запишем формулы для обратного отображения $\mathscr{S}_{\varepsilon}^{-1}$ :

$$
\mathscr{S} \mathscr{M}_{\varepsilon}^{-1}\left(\begin{array}{l}
x \\
y \\
s
\end{array}\right)=\left(\begin{array}{c}
x^{-} \\
y^{-} \\
s^{-}
\end{array}\right)=\left(\begin{array}{c}
x-\frac{1+O(\varepsilon)}{\lambda}\left(\ln \frac{\varepsilon}{\alpha_{s^{-}}^{2} \lambda}+\ln |y|\right) \\
y-\lambda \nu_{s^{-}}\left(x^{-}-\left(\ln \alpha_{s^{-}}\right) / \lambda\right)+O(\varepsilon) \\
s \cdot \operatorname{sign}(y)
\end{array}\right) .
$$


ПрЕДЛОЖЕНИЕ 8.2. Обратимость отображения $T_{\varepsilon}$ порождает обратимость сепаратрисного отображения. Соответствующая инволюиия в координатах $(x \bmod 1, y, s)$ имеет вид

$$
I_{\varepsilon}\left(\begin{array}{l}
x \\
y \\
s
\end{array}\right)=\left(\begin{array}{c}
-x+2\left(\ln \alpha_{s}\right) / \lambda+O(\varepsilon) \\
y-\lambda \nu_{s}\left(-x+\left(\ln \alpha_{s}\right) / \lambda\right)+O(\varepsilon) \\
s
\end{array}\right) .
$$

Функиии $\nu_{ \pm}$являются нечетными: $\nu_{ \pm}(x)=-\nu_{ \pm}(-x)$.

ДокАЗАТЕЛьство. В нормальных координатах сепаратрисное отображение имеет вид (3.12), поэтому имеем:

$$
\begin{aligned}
\mathscr{S}_{\varepsilon}^{-1} & =\mathscr{G}_{\varepsilon}^{-1} \circ \mathscr{T}_{\varepsilon}^{-n_{r}}=\mathscr{G}_{\varepsilon}^{-1} \circ \mathscr{I}_{\varepsilon} \circ \mathscr{T}_{\varepsilon}^{n_{r}} \circ \mathscr{I}_{\varepsilon} \\
& =\mathscr{G}_{\varepsilon}^{-1} \circ \mathscr{I}_{\varepsilon} \circ \mathscr{T}_{\varepsilon}^{n_{r}} \circ \mathscr{G}_{\varepsilon} \circ \mathscr{G}_{\varepsilon}^{-1} \circ \mathscr{I}_{\varepsilon}=\mathscr{G}_{\varepsilon}^{-1} \circ \mathscr{I}_{\varepsilon} \circ \mathscr{S}_{\mathscr{M}_{\varepsilon}} \circ \mathscr{G}_{\varepsilon}^{-1} \circ \mathscr{I}_{\varepsilon} .
\end{aligned}
$$

Поскольку $\mathscr{I}_{\varepsilon} \circ \mathscr{G}_{\varepsilon}^{-1}=\mathscr{G}_{\varepsilon} \circ \mathscr{I}_{\varepsilon}$, получаем, что в нормальных координатах $\mathscr{S}_{\varepsilon}$ обратимо относительно инволюции $\mathscr{I}_{\varepsilon} \circ \mathscr{G}_{\varepsilon}^{-1}$.

Используя (3.11) и (8.1), получаем:

$$
\left(\mathscr{G}_{\varepsilon}\right)^{-1} \circ \mathscr{I}_{\varepsilon}\left(\begin{array}{l}
q \\
p
\end{array}\right)=\left(\begin{array}{c}
\alpha_{s}^{2} / q+O(\varepsilon, p) \\
p q^{2} \alpha_{s}^{-2}-\varepsilon q \nu_{s}(\ln |q| / \lambda)+O_{2}(\varepsilon, p)
\end{array}\right) .
$$

Записывая эту систему в координатах $(x \bmod 1, y, s)$, получим (8.6). Нечетность функций $\nu_{ \pm}(x)$ следует из тождества $I_{\varepsilon}^{2}=\mathrm{id}$.

\section{§ 2. Симметричные траектории периода 3}

Чтобы использовать обратимость, мы будем искать ЭПЛ-траектории, симметричные относительно инволюций $I_{\varepsilon}$. Пусть $z_{0}=\left(x_{0}, y_{0},-1\right)$ - начальное условие для периодической траектории отображения $\mathscr{S}_{\varepsilon}$ периода 3 . Предположим, что $z_{0}$ лежит на оси симметрии, т.е. $I_{\varepsilon}\left(z_{0}\right)=z_{0}$. Тогда согласно (8.6) имеем: $x_{0}=\lambda^{-1} \ln \alpha_{-}+O(\varepsilon)$.

Запишем условие периодичности в виде $\mathscr{S}_{\varepsilon}^{-1}\left(z_{0}\right)=\mathscr{S}_{\mathcal{\varepsilon}}^{2}\left(z_{0}\right)$ :

$$
\left\{\begin{array}{l}
\frac{\ln \alpha_{+}}{\lambda}-\gamma+O(\varepsilon)=\gamma+\frac{1}{\lambda}\left(\ln \frac{\varepsilon}{\alpha_{+} \lambda}+\ln \left(y_{0}+\lambda \nu_{+}(\gamma)\right)\right)+O(\varepsilon)-m, \\
y_{0}-\lambda \nu_{+}(-\gamma)+O(\varepsilon)=y_{0}+\lambda \nu_{+}(\gamma)+O(\varepsilon), \\
0<-\operatorname{sign}\left(y_{0}\right)=-\operatorname{sign}\left(y_{0}\right) \operatorname{sign}\left(y_{0}+\alpha_{+}^{2} \nu_{+}(\gamma)\right),
\end{array}\right.
$$

Здесь натуральное число $m$ - период траектории отображения $T_{\varepsilon}$ с начальным условием $z_{0}$ и

$$
\gamma=\frac{1}{\lambda} \ln \frac{\varepsilon\left|y_{0}\right|}{\alpha_{-} \alpha_{+} \lambda} .
$$

Из обратимости отображения и симметрии траектории следует, что первые два уравнения зависимы. Они эквивалентны следующему:

$$
1+\mu^{\kappa-m-3 \sigma}=\mu^{r} \nu_{+}(\sigma)+O(\varepsilon),
$$

где мы положили

$$
y_{0}=-\lambda \mu^{-r}, \quad \varepsilon=\alpha_{+}^{2} \mu^{\sigma-r+\kappa}, \quad \alpha_{+} / \alpha_{-}=\mu^{\kappa} .
$$




\section{§ 3. Условия устойчивости}

В этом параграфе мы вычисляем след матрицы монодромии $M$, соответствующей рассматриваемой периодической траектории. Условие эллиптичности имеет вид $|\operatorname{tr} M|<2$.

Матрица $M$ является дифференциалом отображения $\mathscr{S}_{\varepsilon}^{3}$ в точке $z_{0}$. Пренебрегая членами вида $O(\varepsilon)$, положим:

$$
\begin{aligned}
& z_{1}=\mathscr{S}_{\varepsilon}\left(z_{0}\right)=\left(\begin{array}{c}
\frac{1}{\lambda} \ln \frac{\varepsilon\left|y_{0}\right|}{\alpha_{-} \lambda} \\
y_{0} \\
1
\end{array}\right), \\
& z_{2}=\mathscr{S}_{\varepsilon}^{2}\left(z_{0}\right)=\mathscr{S}_{\mathcal{E}}^{-1}\left(z_{0}\right)=\left(\begin{array}{c}
-\frac{1}{\lambda} \ln \frac{\varepsilon\left|y_{0}\right|}{\alpha_{-} \alpha_{+}^{2} \lambda} \\
y_{0}+\lambda \nu_{+}(\gamma) \\
1
\end{array}\right) .
\end{aligned}
$$

Дифференциал отображения $\mathscr{S} \mathscr{M} \varepsilon$ имеет вид

$$
D \mathscr{S} \mathscr{M}_{\varepsilon}(z)=\left(\begin{array}{cc}
1+\lambda \nu_{s}^{\prime}\left(x-\ln \alpha_{s} / \lambda\right) / y^{+} & 1 / y^{+} \\
\lambda \nu_{s}^{\prime}\left(x-\ln \alpha_{s} / \lambda\right) & 1
\end{array}\right)
$$

где $z=(x, y, s), y^{+}=y+\lambda \nu_{s}\left(x-\ln \alpha_{s} / \lambda\right)$.

Величину $\operatorname{tr} M=\operatorname{tr}\left(D \mathscr{S}_{\mathcal{\varepsilon}}\left(z_{2}\right) D \mathscr{S}_{\varepsilon}\left(z_{1}\right) D \mathscr{S}_{\varepsilon}\left(z_{0}\right)\right)$ с помощью (8.8)-(8.12) можно представить в виде

$$
\begin{aligned}
\operatorname{tr} M & =-2+\delta \Lambda+O(\varepsilon), \\
\Lambda & =\mu^{r-\kappa+m+3 \sigma} \nu_{-}^{\prime}(0)+\left(2-\mu^{r} \nu_{-}^{\prime}(0)\right)\left(2+\mu^{r-\kappa+m+3 \sigma} \nu_{+}^{\prime}(\sigma)\right), \\
\delta & =1-\mu^{r} \nu_{+}^{\prime}(\sigma) .
\end{aligned}
$$

\section{§ 4. ЭПЛ-траектории существуют}

Итак, условие существования симметричной ЭПЛ-траектории периода 3 имеет вид

$$
\begin{gathered}
1+\mu^{\kappa-m-3 \sigma}=\mu^{r} \nu_{+}(\sigma)+O(\varepsilon), \\
|-2+\delta \Lambda+O(\varepsilon)|<2 .
\end{gathered}
$$

ПреДлОЖЕНИЕ 8.3. Предположим, что переменные $(m, r, \sigma), m \in \mathbf{N}$, удовлетворяют системе (8.14)-(8.15) с опущенными членами $O(\varepsilon)$. Тогда переменные $(m+3, r, \sigma-1)$ также удовлетворяют этой системе.

СлЕдСтвиЕ 8.4. Множество значений параметра $\varepsilon>0$, для которых существует ЭПЛ-траектория, задаваемая с помощью (8.14)-(8.15), асимптотически инвариантно при сжатии $\varepsilon \mapsto \varepsilon / \mu$.

СлЕдСтвиЕ 8.5. Чтобы доказать теорему 1.3, достаточно установить существование одного решения системы (8.14)-(8.15) $\left.\right|_{O(\varepsilon)=0}$. 
Следствие 8.4 очевидно. Чтобы доказать следствие 8.5, предположим, что система $(8.14)-\left.(8.15)\right|_{O(\varepsilon)=0}$ имеет решение $\left(m_{0}, r_{0}, \sigma_{0}\right)$. Так как $(8.15)$ - неравенство, система $(8.14)-\left.(8.15)\right|_{O(\varepsilon)=0}$ имеет при $m=m_{0}$ криволинейный интервал решений на плоскости $r, \sigma$. В частности, существует интервал $\left(\varepsilon_{1}, \varepsilon_{2}\right)$, лежащий в $J$. Согласно следствию 8.4 множество $J$ содержит большую часть (в смысле меры) точек множества $\bigcup_{n=0}^{\infty}\left(\mu^{-n} \varepsilon_{1}, \mu^{-n} \varepsilon_{2}\right)$. Отсюда следует теорема 1.3.

ПреДлОЖениЕ 8.6. Для любого $\kappa \in \mathbb{R}$ система $(8.14)-\left.(8.15)\right|_{O(\varepsilon)=0}$ uмеет решение.

ДокАЗАтЕЛьСтво. Зафиксируем $\kappa$ и положим в (8.14)-(8.15) $O(\varepsilon)=0$. Напомним, что периодические функции $\nu_{ \pm}$имеют нулевое среднее. Так как сепаратрисы $T_{\varepsilon}$ расщепляются в первом приближении по $\varepsilon$, то по крайней мере одна из функций $\nu_{ \pm}$не равна тождественному нулю. Мы предположим, что $\nu_{+} \not \equiv 0$. (Иначе во всех предыдущих формулах поменяем местами + и -.)

Для любого $m \in \mathbb{Z}$ на полосе $\left\{(\sigma, r) \in \mathbb{R}^{2}: \sigma \in[0,2 \pi]\right\}$ имеется гладкая кривая $\Phi_{m}$ - решение уравнения (8.14). Кривые $\Phi_{m}$ можно считать ориентированными. Пусть $\partial$ - оператор дифференцирования вдоль $\Phi_{m}$. Элементарно устанавливается, что

(a) на каждой из кривых $\Phi_{m}$ имеется точка $\left(\sigma_{m}, r_{m}\right)$, в которой $\delta=0$;

(b) равенство $\Lambda(\sigma, r)=0$ имеет место не более чем в конечном числе точек $\left(\sigma_{m}, r_{m}\right)$

(c) равенство $\partial \delta(\sigma, r)=0$ также имеет место не более чем в конечном числе точек $\left(\sigma_{m}, r_{m}\right)$.

Пусть $\left(\sigma_{m}, r_{m}\right) \in \Phi_{m}$ - точка, в которой $\Lambda \neq 0$ и $\partial \delta \neq 0$. Тогда согласно (8.15)

$$
\operatorname{tr} M\left(\sigma_{m}, r_{m}\right)=-2, \quad \partial(\operatorname{tr} M)\left(\sigma_{m}, r_{m}\right) \neq 0 .
$$

Следовательно, в малой окрестности точки $\left(\sigma_{m}, r_{m}\right)$ на $\Phi_{m}$ имеются точки, в которых $|\operatorname{tr} M|<2$.

Доказательство теоремы 1.1. Отметим, что любое интегрируемое отображение $T_{0}$ обратимо относительно некоторой инволюции, сохраняющей петли. Включим $T_{0}$ в семейство $T_{\varepsilon}$, обратимое относительно инволюции, сохраняющей петли. Рассмотрим семейство симплектических отображений $\widehat{T}_{\varepsilon}$ такое, что

$$
T_{0}=\widehat{T}_{0}, \quad\left|d T_{\varepsilon} / d \varepsilon\right|_{\varepsilon=0}-d \widehat{T}_{\varepsilon} /\left.d \varepsilon\right|_{\varepsilon=0} \mid=\Delta
$$

для некоторого малого $\Delta>0$. Сепаратрисное отображение, соответствующее $\widehat{T}_{\varepsilon}$, также имеет вид $(8.5)$, но периодические функции $\widehat{\nu}_{ \pm}$, играющие роль функций $\nu_{ \pm}$, вообще говоря, уже не являются нечетными. Однако они $O(\Delta)$ близки к нечетным функциям $\nu_{ \pm}$:

$$
\left|\widehat{\nu}_{+}-\nu_{+}\right| \sim \Delta, \quad\left|\widehat{\nu}_{-}-\nu_{-}\right| \sim \Delta .
$$

Если $\Delta$ мало, то ЭПЛ-траекторию в $T_{\varepsilon}$ можно продолжить до ЭПЛ-траектории отображения $\widehat{T}_{\varepsilon}$. Так как множество $J$ для отображений $T_{\varepsilon}$ состоит из интервалов, то существует интервал $\left(\widehat{\varepsilon}_{1}, \widehat{\varepsilon}_{2}\right)$ такой, что для любого $\varepsilon \in\left(\widehat{\varepsilon}_{1}, \widehat{\varepsilon}_{2}\right)$ отображение $\widehat{T}_{\varepsilon}$ имеет ЭПЛ-траекторию. Теперь теорема 1.1 вытекает из следующего простого утверждения. 
Предположим, что отображение $T_{\varepsilon}$ имеет ЭПЛ-траекторию для некоторого малого є. Тогда $T_{\varepsilon / \mu}$ также имеет ЭПЛ-траекторию (большего периода). Это утверждение аналогично следствию 8.4 и вытекает из уравнений (8.5).

\section{§ 5. Площадь островка устойчивости}

Площадь островка устойчивости вокруг ЭПЛ-траектории имеет тот же порядок малости, что и площадь сепаратрисной лунки, так как в переменных $(x, y)$ обе оказываются порядка единицы.

\section{Глава 9. Рост энергии в задаче Мезера}

В этой главе метод сепаратрисного отображения применяется к задаче Мезера (§4 главы 1) для построения траекторий, на которых энергия в среднем растет линейно по времени.

\section{§ 1. Формулировка результата}

Предположим, что система (5.1)-(5.2) удовлетворяет следующим условиям Мезера.

$\mathbf{H}_{\mathbf{0}}$. Невозмущенная система имеет периодическую гиперболическую траекторию $\sigma \subset \mathscr{M}$ и трансверсальное гомоклиническое $\kappa$ ней решение $\gamma: \mathbb{R} \rightarrow \mathscr{M}$.

В частности, для системы (5.1)-(5.2) верна теорема 5.4.

$\mathbf{H}_{\mathbf{1}}$. Интеграл Пуанкаре-Мельникова (5.6) - непостоянная функция.

ТеОрема 9.1. Пусть для системы (5.1)-(5.2) выполнены условия $\mathbf{H}_{\mathbf{0}} u \mathbf{H}_{\mathbf{1}}$. Тогда существует решение $\eta(t)$ этой системы такое, что при всех $t \geqslant 0$

$$
\mathscr{H}(\eta(t), t) \geqslant A^{-} t+B^{-}
$$

для некоторых постоянных $A^{-}>0$ и $B^{-}$.

ЗАмечание 9.2. Пусть $\frac{\partial}{\partial t} \mathscr{H}_{1}(\mathscr{Z}, t) \leqslant A^{+}$. Например, это выполнено, когда поверхности уровня $\mathscr{H}_{0}$ компактны, что, конечно, имеет место в задаче Мезера. Тогда для любого решения $\eta(t)$ системы (5.1)

$$
\frac{d \mathscr{H}(\eta(t), t)}{d t}=\frac{\partial \mathscr{H}_{1}(\eta(t), t)}{\partial t} \leqslant A^{+},
$$

и, следовательно,

$$
\mathscr{H}(\eta(t), t) \leqslant A^{+} t+B^{+} .
$$

Таким образом, рост энергии более быстрый, чем линейный по времени, невозможен ни на каком решении. В этом смысле оценка (9.1) точна.

Мы докажем теорему 9.1, используя формулы для сепаратрисного отображения задачи Мезера (теорема 5.4), т.е. $\eta(t)$ ищется среди решений, которые 
всегда остаются в окрестности семейства невозмущенных гомоклинических решений $\gamma_{\mu}(t)$ (см. главу 5). Для доказательства теоремы 9.1 достаточно построить траекторию сепаратрисного отображения

$$
\left\{\left(x_{n}, y_{n}, t_{n}, h_{n}\right)\right\}_{n \in \mathbb{Z}}, \quad \mathscr{S} \mathscr{M}\left(x_{n}, y_{n}, t_{n}, h_{n}\right)=\left(x_{n+1}, y_{n+1}, t_{n+1}, h_{n+1}\right)
$$

такую, что

$$
h_{n} \geqslant A^{-} t_{n}+B
$$

для некоторой постоянной $B$.

\section{§ 2. Символические траектории}

В этом параграфе мы построим сжимающий оператор на пространстве последовательностей, неподвижными точками которого являются траектории сепаратрисного отображения. Эти траектории имеют хаотический характер в том смысле, что они допускают кодирование последовательностями целых чисел (предложение 9.5).

Обозначим через $[x]$ округление вещественного числа $x$ до целого, $\{x\}=x-$ $[x]$ - "расстояние со знаком" до ближайшего целого числа. Например, $[2.9]=3$, $\{2.9\}=-0.1$.

Сепаратрисное отображение имеет естественное поднятие на накрывающую область

$$
\begin{aligned}
Q_{*}=\{(x, y, t, h) & \left.\in \mathbb{R}^{4}: \frac{1}{\sqrt[4]{h}}<y, \frac{1}{\sqrt[4]{h}}<Y ;\{x\}, y-\text { малы }\right\} \\
Y & =a\{x\}+b y+\boldsymbol{O}_{2}(\{x\}, y)
\end{aligned}
$$

Это отображение, которое мы снова обозначим $\mathscr{S} \mathscr{M}$, также имеет вид (5.9), где $\boldsymbol{O}_{1}=\boldsymbol{O}_{1}(Y \ln Y)+\boldsymbol{O}_{1}(\{x\}, y)$ на $Q_{*}$ и мы можем считать, что $x_{0} \in\left(-\frac{1}{2}, \frac{1}{2}\right]$.

Лемма 9.3. Система $\mathscr{S} \mathscr{M}(x, y, t, h)=\left(x^{+}, y^{+}, t^{+}, h^{+}\right)$равносилъна следующей:

$$
\left\{\begin{array}{l}
y^{+}=e^{-\frac{\lambda}{\nu}\left(x^{+}-x-x_{0}+R^{y}\right)}, \\
a\{x\}=y^{+}-b y+R^{x}, \\
t^{+}=t+\frac{1}{\nu \sqrt{h}}\left(x^{+}-x-x_{0}+R^{t}\right), \\
h^{+}=h+\frac{1}{\sqrt{h}}\left(I^{\prime}(t)+R^{h}\right), \\
Y(x, y, t, h)=e^{-\frac{\lambda}{\nu}\left(x^{+}-x-x_{0}+R^{Y}\right)} .
\end{array}\right.
$$

Здесъ $R^{x, y, Y, t, h}(x, y, t, h)$ - гладкие функиии на $Q_{*}$ такие, что

$$
\begin{aligned}
& R^{y, Y, t, h}(x, y, t, h)=\boldsymbol{O}\left(\left(x^{+}-x\right) e^{-\frac{\lambda}{\nu}\left(x^{+}-x\right)}\right)+\boldsymbol{O}_{1}(\{x\}, y) \quad \text { на } Q_{*}, \\
& R^{x}(x, y, t, h)=\boldsymbol{O}\left(\left(x^{+}-x\right) e^{-\frac{2 \lambda}{\nu}\left(x^{+}-x\right)}\right)+\boldsymbol{O}_{2}(\{x\}, y) \quad \text { на } Q_{*} .
\end{aligned}
$$


ДоказАТЕЛЬСтво. Достаточно проверить (9.4). Из первого уравнения системы (5.9) следует, что

$$
x^{+}=x+x_{0}-\frac{\nu}{\lambda}(1+\boldsymbol{O}(Y)) \ln Y+\widetilde{R}, \quad \widetilde{R}(x, y, t, h)=\boldsymbol{O}_{1}(\{x\}, y) .
$$

Положим $\widetilde{Y}=e^{-\frac{\lambda}{\nu}\left(x^{+}-x-x_{0}-\widetilde{R}\right)}$. Тогда

$$
\begin{gathered}
\frac{\nu}{\lambda} \ln \tilde{Y}=\frac{\nu}{\lambda}(1+\boldsymbol{O}(Y)) \ln Y, \quad \tilde{Y}=Y+\boldsymbol{O}\left(Y^{2} \ln Y\right), \\
Y=\widetilde{Y}+\boldsymbol{O}\left(\tilde{Y}^{2} \ln \tilde{Y}\right)=\tilde{Y}(1+\boldsymbol{O}(\tilde{Y} \ln \tilde{Y}))=e^{-\frac{\lambda}{\nu}\left(x^{+}-x-x_{0}+\widetilde{R}+\boldsymbol{O}(\tilde{Y} \ln \tilde{Y})\right)} .
\end{gathered}
$$

Лемма 9.3 доказана.

Нам будет необходима следующая регуляризация сепаратрисного отображения. Для $E>0$ положим

$$
\mathscr{S}_{E}(x, y, t, h)=\left(x^{+}, y^{+}, t^{+}, h^{+}\right)
$$

где

$$
\begin{gathered}
x^{+}=x_{\mathscr{S M}}^{+}, \quad y^{+}=y_{\mathscr{S M}}^{+}, \quad t^{+}=t_{\mathscr{S M}}^{+}, \quad h^{+}=\max \left\{E, h_{\mathscr{S M}}^{+}\right\}, \\
\left(x_{\mathscr{S M}}^{+}, y_{\mathscr{S} \mathscr{M}}^{+}, t_{\mathscr{S} \mathscr{M}}^{+}, h_{\mathscr{S M}}^{+}\right)=\mathscr{S} \mathscr{M}(x, y, t, h) .
\end{gathered}
$$

ЗАмЕЧАНИЕ 9.4. Пусть

$$
\mathscr{S}_{E}\left(x_{n}, y_{n}, t_{n}, h_{n}\right)=\left(x_{n+1}, y_{n+1}, t_{n+1}, h_{n+1}\right), \quad n \in \mathbb{Z},
$$

и $h_{n}>E$ для всех $n \in \mathbb{Z}$. Тогда $\left\{\left(x_{n}, y_{n}, t_{n}, h_{n}\right)\right\}_{n \in \mathbb{Z}}-$ траектория отображения $\mathscr{S} \mathscr{M}$.

Для числовых последовательностей $\boldsymbol{a}^{\prime}=\left\{a_{n}^{\prime}\right\}_{n \in \mathbb{Z}}$ и $\boldsymbol{a}^{\prime \prime}=\left\{a_{n}^{\prime \prime}\right\}_{n \in \mathbb{Z}}$ определим

$$
\operatorname{dist}\left(\boldsymbol{a}^{\prime}, \boldsymbol{a}^{\prime \prime}\right)=\sup _{n \in \mathbb{Z}}\left\|a_{n}^{\prime}-a_{n}^{\prime \prime}\right\|_{(n)},
$$

где $\|a\|_{(n)}=2^{-|n|}|a|, a \in \mathbb{R}$.

Обозначим

$$
\begin{gathered}
\boldsymbol{x}=\left\{x_{n}\right\}_{n \in \mathbb{Z}}, \quad \boldsymbol{y}=\left\{y_{n}\right\}_{n \in \mathbb{Z}}, \quad \boldsymbol{t}=\left\{t_{n}\right\}_{n \in \mathbb{Z}}, \quad \boldsymbol{h}=\left\{h_{n}\right\}_{n \in \mathbb{Z}}, \\
\zeta=(x, y, t, h), \quad \boldsymbol{\zeta}=\left\{\zeta_{n}\right\}_{n \in \mathbb{Z}}=(\boldsymbol{x}, \boldsymbol{y}, \boldsymbol{t}, \boldsymbol{h}), \\
\operatorname{dist}_{\mathfrak{Z}}\left(\boldsymbol{\zeta}^{\prime}, \boldsymbol{\zeta}^{\prime \prime}\right)=\max \left\{\operatorname{dist}\left(\boldsymbol{x}^{\prime}, \boldsymbol{x}^{\prime \prime}\right), \operatorname{dist}\left(\boldsymbol{y}^{\prime}, \boldsymbol{y}^{\prime \prime}\right), \operatorname{dist}\left(\boldsymbol{t}^{\prime}, \boldsymbol{t}^{\prime \prime}\right), \operatorname{dist}\left(\boldsymbol{h}^{\prime}, \boldsymbol{h}^{\prime \prime}\right)\right\} .
\end{gathered}
$$

Пространство $\mathfrak{Z}=\left(\{\boldsymbol{\zeta}\}, \operatorname{dist}_{\mathfrak{Z}}\right)$ является полным метрическим пространством.

Для $K^{\prime}, K^{\prime \prime} \in \mathbb{N}, K^{\prime \prime}>K^{\prime}+1$ (на самом деле ниже $K^{\prime}$ и $K^{\prime \prime}$ достаточно велики и $K^{\prime \prime} \gg K^{\prime}$ ) определим пространство кодов

$$
\begin{gathered}
\mathscr{C}_{K^{\prime}, K^{\prime \prime}}=\left\{\overline{\boldsymbol{x}}=\left\{\bar{x}_{n}\right\}_{n \in \mathbb{Z}}: \bar{x}_{n} \in \mathbb{Z}, K^{\prime}<\bar{x}_{n+1}-\bar{x}_{n}<K^{\prime \prime}\right\}, \\
\operatorname{dist}_{C}\left(\overline{\boldsymbol{x}}^{\prime}, \overline{\boldsymbol{x}}^{\prime \prime}\right)=2^{-N}, \quad N=\min \left\{|n|: \bar{x}_{n}^{\prime} \neq \bar{x}_{n}^{\prime \prime}\right\} .
\end{gathered}
$$


ПРЕДЛОЖЕНИЕ 9.5. Пусть є достаточно мало и

$$
\frac{1}{\sqrt[4]{E}}<e^{-\frac{\lambda}{\nu} K^{\prime \prime}}, \quad\left(\frac{1+|b|}{|a|}+1\right) e^{-\frac{\lambda}{\nu} K^{\prime}}<\frac{1}{2} \varepsilon .
$$

Тогда для любого кода $\overline{\boldsymbol{x}} \in \mathscr{C}_{K^{\prime}, K^{\prime \prime}}$ u чисел $t_{0}, h_{0} \in \mathbb{R}, h_{0} \geqslant E$, существует единственная траектория $\boldsymbol{\zeta}=\left\{\left(x_{n}, y_{n}, t_{n}, h_{n}\right)\right\}_{n \in \mathbb{Z}}$ отображения $\mathscr{S}_{E}$ такая, что

$$
\left|x_{n}-\bar{x}_{n}\right| \leqslant \varepsilon, \quad \frac{1}{\sqrt[4]{E}} \leqslant y_{n} \leqslant \varepsilon, \quad \frac{1}{\sqrt[4]{E}} \leqslant Y_{n} \leqslant \varepsilon, \quad h_{n} \geqslant E .
$$

При этом, если $\boldsymbol{\zeta}^{\prime}$ (соответственно $\left.\boldsymbol{\zeta}^{\prime \prime}\right)$ порождает траекторию $\overline{\boldsymbol{x}}^{\prime}$ (соответственно $\left.\overline{\boldsymbol{x}}^{\prime \prime}\right) \in \mathscr{C}_{K^{\prime}, K^{\prime \prime}}$, то

$$
\operatorname{dist}\left(\boldsymbol{t}^{\prime}, \boldsymbol{t}^{\prime \prime}\right) \leqslant 8 \varepsilon \operatorname{dist}_{C}\left(\overline{\boldsymbol{x}}^{\prime}, \overline{\boldsymbol{x}}^{\prime \prime}\right) .
$$

Таким образом, при фиксированных $t_{0}, h_{0}>E$ получаем отображение из пространства кодов в пространство траекторий:

$$
\mathscr{C} \mathscr{M}: \overline{\boldsymbol{x}} \in \mathscr{C}_{K^{\prime}, K^{\prime \prime}} \mapsto \boldsymbol{\zeta}=\left\{\left(x_{n}, y_{n}, t_{n}, h_{n}\right)\right\}_{n \in \mathbb{Z}},
$$

где $\mathscr{S}_{E}\left(x_{n}, y_{n}, q_{n}, p_{n}\right)=\left(x_{n+1}, y_{n+1}, t_{n+1}, h_{n+1}\right)$.

ДокАЗАТЕЛЬСтво. Зафиксируем произвольный код $\overline{\boldsymbol{x}} \in C_{K^{\prime}, K^{\prime \prime}}$ и выберем $t_{0}, h_{0} \geqslant E$. В пространстве последовательностей $\mathfrak{Z}$ рассмотрим множество

$$
\begin{aligned}
& \mathrm{B}_{\varepsilon, E}=\left\{\zeta=\left\{\zeta_{n}\right\}_{n \in \mathbb{Z}}:\right.\left|x_{n}-\bar{x}_{n}\right| \leqslant \varepsilon, \frac{1}{\sqrt[4]{E}} \leqslant y_{n} \leqslant \varepsilon \\
&\left.\frac{1}{\sqrt[4]{E}} \leqslant Y_{n} \leqslant \varepsilon, h_{n} \geqslant E ; t_{0}, h_{0} \text { фиксированы }\right\} .
\end{aligned}
$$

Для достаточно малого $\varepsilon: \mathrm{B}_{\varepsilon, E} \neq \varnothing$ и все точки $\left(x_{n}, y_{n}, t_{n}, h_{n}\right)$ лежат в области $Q_{*}$ - области определения $\mathscr{S}_{E}$. Поэтому на этом множестве определен оператор $\mathbf{C}: \mathrm{B}_{\varepsilon, E} \rightarrow \mathfrak{Z}$,

$$
\begin{aligned}
& \mathbf{C}: \boldsymbol{\zeta}=\left\{\left(x_{n}, y_{n}, t_{n}, h_{n}\right)\right\}_{n \in \mathbb{Z}} \longmapsto \widetilde{\boldsymbol{\zeta}}=\left\{\left(\widetilde{x}_{n}, \widetilde{y}_{n}, \widetilde{t}_{n}, \widetilde{h}_{n}\right)\right\}_{n \in \mathbb{Z}}, \\
& {\left[\widetilde{x}_{n}\right]=\bar{x}_{n}, \quad \widetilde{t}_{0}=t_{0}, \quad \widetilde{h}_{0}=h_{0},} \\
& a\left\{\widetilde{x}_{n}\right\}=e^{-\frac{\lambda}{\nu}\left(x_{n+1}-x_{n}-x_{0}+R_{n}^{y}\right)}-b e^{-\frac{\lambda}{\nu}\left(x_{n}-x_{n-1}-x_{0}+R_{n-1}^{y}\right)}+R_{n}^{x}, \\
& \widetilde{y}_{n}=e^{-\frac{\lambda}{\nu}\left(x_{n}-x_{n-1}-x_{0}+R_{n}^{y}\right)}, \\
& \widetilde{t}_{n}= \begin{cases}t_{n-1}+\frac{1}{\nu \sqrt{h_{n-1}}}\left(x_{n}-x_{n-1}-x_{0}+R_{n-1}^{t}\right) & \text { при } n>0, \\
t_{n+1}-\frac{1}{\nu \sqrt{h_{n}}}\left(x_{n+1}-x_{n}-x_{0}+R_{n}^{t}\right) & \text { при } n<0,\end{cases} \\
& \widetilde{h}_{n}= \begin{cases}\max \left\{E, h_{n-1}+\frac{1}{\sqrt{h_{n-1}}}\left(I^{\prime}\left(t_{n-1}\right)+R_{n-1}^{h}\right)\right\} & \text { при } n>0, \\
\max \left\{E, h_{n+1}-\frac{1}{\sqrt{h_{n+1}}}\left(I^{\prime}\left(t_{n+1}\right)+R_{n}^{h}\right)\right\} & \text { при } n<0 .\end{cases}
\end{aligned}
$$

Из (9.3) нетрудно видеть, что неподвижные точки этого отображения есть траектории отображения $\mathscr{S}_{E}$. 
Лемма 9.6. а) $\mathbf{C}\left(\mathrm{B}_{\varepsilon, E}\right) \subset \mathrm{B}_{\varepsilon, E}$.

b) Oператор $\mathbf{C}$ сжимающий:

$$
\operatorname{dist}_{\mathfrak{Z}}\left(\mathbf{C} \boldsymbol{\zeta}^{\prime}, \mathbf{C} \boldsymbol{\zeta}^{\prime \prime}\right)<\frac{3}{4} \operatorname{dist}_{\mathfrak{Z}}\left(\boldsymbol{\zeta}^{\prime}, \boldsymbol{\zeta}^{\prime \prime}\right)
$$

Доказательство этой леммы имеется в [25]. Заметим, что оператор $\mathbf{C}$ вообще говоря не является сжимающим по норме равномерной сходимости.

Поскольку $\mathrm{B}_{\varepsilon, E}$ - замкнутое подпространство полного пространства $\mathfrak{Z}$, то из принципа сжимающих отображений следует, что $\mathbf{C}$ имеет единственную неподвижную точку $\boldsymbol{\zeta} \in \mathrm{B}_{\varepsilon, E}$ - траекторию сепаратрисного отображения.

Докажем оценку (9.9). Пусть $\boldsymbol{\zeta}^{\prime}$ и $\boldsymbol{\zeta}^{\prime \prime}-$ траектории, соответствующие кодам $\overline{\boldsymbol{x}}^{\prime}$ и $\overline{\boldsymbol{x}}^{\prime \prime} \in \mathscr{C}_{K^{\prime}, K^{\prime \prime}}$ соответственно. Пусть $\operatorname{dist}_{C}\left(\overline{\boldsymbol{x}}^{\prime}, \overline{\boldsymbol{x}}^{\prime \prime}\right)=2^{-N}$. Возьмем в качестве нулевого приближения к траектории $\zeta^{\prime \prime}=\left\{\zeta_{n}^{\prime \prime}\right\}_{n \in \mathbb{Z}}$ следующую последовательность $\zeta^{(0)}=\left\{\zeta_{n}^{(0)}\right\}_{n \in \mathbb{Z}}$ :

$$
x_{n}^{(0)}=\left\{\begin{array}{ll}
x_{n}^{\prime} & \text { при }|n|<N, \\
\bar{x}_{n}^{\prime \prime} & \text { при }|n| \geqslant N,
\end{array} \quad y_{n}^{0}=y_{n}^{\prime}, \quad t_{n}^{(0)}=t_{n}^{\prime}, \quad h_{n}^{(0)}=h_{n}^{\prime} .\right.
$$

Тогда, из определения оператора $\mathbf{C}$, первое приближение $\boldsymbol{\zeta}^{(1)}=\mathbf{C}\left(\boldsymbol{\zeta}^{(0)}\right)$, имеет вид:

$$
\begin{aligned}
x_{n}^{(1)} & =x_{n}^{(0)} & & \text { при }|n|<N-1, \\
\left|x_{n}^{(1)}-x_{n}^{(0)}\right| & \leqslant \varepsilon & & \text { при }|n| \geqslant N-1, \\
y_{n}^{(1)} & =y_{n}^{(0)} & & \text { при }|n|<N-1, \\
\left|y_{n}^{(1)}-y_{n}^{(0)}\right| & \leqslant \varepsilon & & \text { при }|n| \geqslant N-1, \\
t_{n}^{(1)} & =t_{n}^{(0)} & & \text { при }|n|<N, \\
\left|t_{n}^{(1)}-t_{n}^{(0)}\right| & \leqslant \frac{K^{\prime \prime}}{\nu \sqrt{E}} & & \text { при }|n| \geqslant N, \\
h_{n}^{(1)} & =h_{n}^{(0)} & & \text { при }|n|<N, \\
\left|h_{n}^{(1)}-h_{n}^{(0)}\right| & \leqslant \frac{1}{\nu \sqrt{E}}+\frac{K^{\prime \prime} C}{E} & & \text { при }|n| \geqslant N .
\end{aligned}
$$

Поэтому, при необходимости уменьшая $\varepsilon$, получим:

$$
\operatorname{dist}\left(\boldsymbol{\zeta}^{(0)}, \boldsymbol{\zeta}^{(1)}\right) \leqslant 2^{-N} 2 \varepsilon
$$

Следовательно, из свойства сжимающего отображения,

$$
\operatorname{dist}\left(\boldsymbol{\zeta}^{(0)}, \boldsymbol{\zeta}^{\prime \prime}\right) \leqslant \frac{1}{1-\frac{3}{4}} \operatorname{dist}\left(\boldsymbol{\zeta}^{(0)}, \boldsymbol{\zeta}^{(1)}\right) \leqslant 8 \varepsilon 2^{-N} .
$$

Отсюда

$$
\operatorname{dist}\left(\boldsymbol{t}^{\prime}, \boldsymbol{t}^{\prime \prime}\right) \leqslant \operatorname{dist}\left(\boldsymbol{t}^{\prime}, \boldsymbol{t}^{(0)}\right)+\operatorname{dist}\left(\boldsymbol{t}^{(0)}, \boldsymbol{t}^{\prime \prime}\right) \leqslant 8 \varepsilon 2^{-N}=8 \varepsilon \operatorname{dist}_{C}\left(\overline{\boldsymbol{x}}^{\prime}, \overline{\boldsymbol{x}}^{\prime \prime}\right) .
$$




\section{§3. Быстрые траектории Мезера}

Траектории с быстрым ростом энергии ищем среди траекторий, построенных в предложении 9.5. Из (9.3) следует, что для траектории $(\boldsymbol{x}, \boldsymbol{y}, \boldsymbol{t}, \boldsymbol{h})$, порожденной кодом $\overline{\boldsymbol{x}}$, имеем

$$
\delta h_{n}=\frac{\nu I^{\prime}\left(t_{n}\right)}{\bar{x}_{n+1}-\bar{x}_{n}-x_{0}} \delta t_{n}+\text { малые ошибки, }
$$

где $\delta h_{n}=h_{n+1}-h_{n}$ и $\delta t_{n}=t_{n+1}-t_{n}$. Теперь, если мы выберем код такой, что разность $\bar{x}_{n+1}-\bar{x}_{n}$ поменьше при $I^{\prime}\left(t_{n}\right)>0$ и побольше при $I^{\prime}\left(t_{n}\right)<0$, то в среднем последовательность $h_{n}$ будет расти при $n \rightarrow \pm \infty$. Следующая лемма показывает, что такой код существует.

Обозначим П: $(\boldsymbol{x}, \boldsymbol{y}, \boldsymbol{t}, \boldsymbol{h}) \mapsto \boldsymbol{t}$ и $\mathscr{C} \mathscr{M}_{t}=\Pi \circ \mathscr{C} \mathscr{M}$. Пусть $\left\{\Theta_{j}\right\}_{j \in \mathbb{Z}}-$ монотонно возрастающая последовательность. Положим $\Delta_{j}=\left[\Theta_{2 j}, \Theta_{2 j+1}\right)$ и $\Xi_{j}=\left[\Theta_{2 j-1}, \Theta_{2 j}\right)$ (см. рис. 9.1).

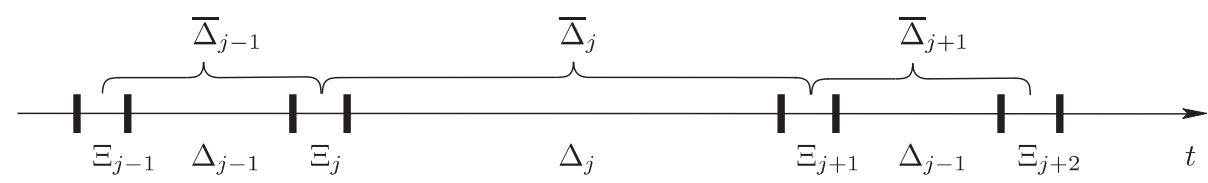

Рис. 9.1. Разбиение $\mathbb{R}_{t}$

ЛЕмма 9.7. Пустъ ${ }^{11}\left|\Xi_{j}\right| \geqslant 8 \varepsilon$ для всех $j \in \mathbb{Z}$. Тогда для любой последовательности $\left\{k_{j}\right\}, k_{j} \in \mathbb{N}, K^{\prime}<k_{j}<K^{\prime \prime}$, существует код $\overline{\boldsymbol{x}} \in C_{K^{\prime}, K^{\prime \prime}}$, $\boldsymbol{t}=\mathscr{C} \mathscr{M}_{t}(\overline{\boldsymbol{x}})$ такой, что

$$
t_{n} \in \Delta_{j} \Rightarrow \begin{cases}\bar{x}_{n+1}-\bar{x}_{n}=k_{j} & \text { npu } n \geqslant 0, \\ \bar{x}_{n}-\bar{x}_{n-1}=k_{j} & \text { npu } n \leqslant 0 .\end{cases}
$$

ДокАЗАТЕЛЬСтво. Искомый код строится по индукции. Пусть

$$
\bar{\Theta}_{j}=\frac{\Theta_{2 j}+\Theta_{2 j-1}}{2}, \quad \bar{\Delta}_{j}=\left[\bar{\Theta}_{j+1}, \bar{\Theta}_{j}\right) .
$$

На $N$-м шаге мы определяем точки $\bar{t}_{N}$ и $\bar{t}_{-N}$ такие, что у полученной в конце всей процедуры последовательности $\boldsymbol{t}=\left\{t_{n}\right\}$ члены $t_{N}$ и $t_{-N}$ будут лежать в $4 \varepsilon-$ окрестности этих точек. Отсюда нетрудно видеть, что свойство (9.11) будет следовать из аналогичного свойства для последовательности $\left\{\bar{t}_{n}\right\}$ и интервалов $\bar{\Delta}_{j}$ :

$$
\bar{t}_{n} \in \bar{\Delta}_{j} \Rightarrow \begin{cases}\bar{x}_{n+1}-\bar{x}_{n}=k_{j} & \text { при } n \geqslant 0, \\ \bar{x}_{n}-\bar{x}_{n-1}=k_{j} & \text { при } n \leqslant 0 .\end{cases}
$$

Это свойство есть индуктивное определение кода. Действительно, получаем следующую процедуру.

На нулевом шаге мы берем произвольный код $\overline{\boldsymbol{x}}^{(0)} \in C_{K^{\prime}, K^{\prime \prime}}$ и полагаем $\bar{t}_{0}=t_{0}$.

\footnotetext{
11 Здесь $|\Delta|$ - длина интервала $\Delta$.
} 
Опишем $N$-й шаг. Из $N-1$ шага мы имеем код $\overline{\boldsymbol{x}}^{(N-1)}$ и соответствующую последовательность $\boldsymbol{t}^{(N-1)}$. Определяем

$$
\begin{aligned}
& \bar{x}_{n}^{(N)}= \begin{array}{ll}
\bar{x}_{n}^{(N-1)} & \text { при }|n|<N, \\
\bar{x}_{N-1}^{(N-1)}+k_{j} & \text { при } n=N, \text { если } t_{N-1}^{(N-1)} \in \bar{\Delta}_{j}, \\
\bar{x}_{-(N-1)}^{(N-1)}-k_{j} & \text { при } n=-N, \text { если } t_{-(N-1)}^{(N-1)} \in \bar{\Delta}_{j}, \\
\text { произвольно } & \text { при }|n|>N,
\end{array} \\
& \bar{t}_{N}=\boldsymbol{t}_{N}^{(N)}, \quad \bar{t}_{-N}=\boldsymbol{t}_{-N}^{(N)}, \quad \text { где } \boldsymbol{t}^{(N)}={\mathscr{C} \mathscr{M}_{t}\left(\boldsymbol{x}^{(N)}\right) .}
\end{aligned}
$$

Таким образом, процедура описана.

Далее пусть $\overline{\boldsymbol{x}}-$ предел соответствующей процедуры и $\boldsymbol{t}=\mathscr{C}_{\mathfrak{M}}(\overline{\boldsymbol{x}})$. Имеем

$$
\operatorname{dist}\left(\boldsymbol{t}^{(N)}, \boldsymbol{t}\right)<8 \varepsilon \operatorname{dist}_{C}\left(\overline{\boldsymbol{x}}^{(N)}, \overline{\boldsymbol{x}}\right) \leqslant 8 \varepsilon 2^{-N-1} .
$$

Следовательно,

$$
\left|t_{N}-\bar{t}_{N}\right|=\left|t_{N}-t_{N}^{(N)}\right|<8 \varepsilon 2^{N} 2^{-N-1}=4 \varepsilon .
$$

Лемма 9.7 доказана.

ДоКАЗАТЕЛЬСТВо тЕОРЕмЫ 9.1. МЫ построим траекторию $\mathscr{S}_{E}$ такую, что для постоянных $A^{+}>0$ и $B>E$

$$
h_{n}>A^{+}\left|t_{n}\right|+B \quad \text { для любого } n \in \mathbb{Z},
$$

и тем самым получим траекторию $\mathscr{S} \mathscr{M}$ с линейным по времени ростом энергии.

Из условия $\mathbf{H}_{1}$ теоремы 9.1 и равенства

$$
\int_{\mathbb{T}(t)} I^{\prime}(t) d t=0
$$

следует, что существует интервал $\Delta \subset \mathbb{T}(t),|\Delta|>0$, такой, что

$$
\min _{t \in \Delta} I^{\prime}(t)>m_{+}>0
$$

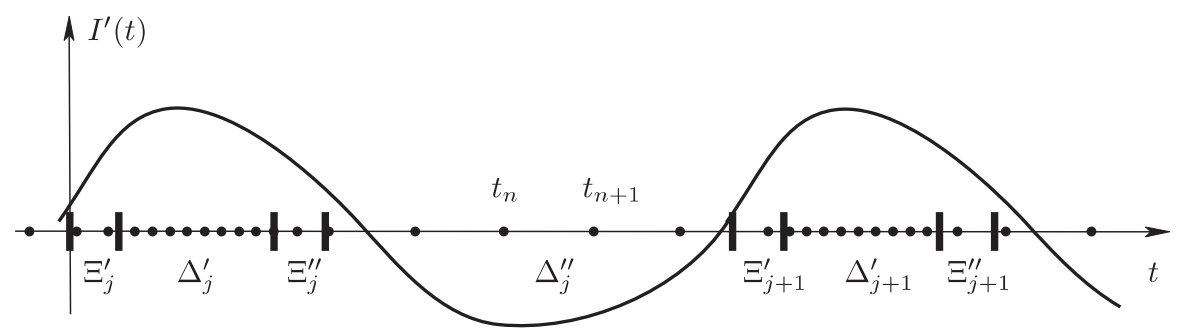

Рис. 9.2. Последовательность $t_{n}$ для траектории с линейным ростом энергии

Положим

$$
\min _{t \in \mathbb{T}} I^{\prime}(t)=-m_{-} .
$$


Пусть $\Delta_{j}^{\prime}, j \in \mathbb{Z},-$ прообразы $\Delta$ при естественном накрытии $\mathbb{R} \rightarrow \mathbb{T}$. Рассмотрим разбиение $\mathbb{R}(t)$ :

$$
\mathbb{R}=\cdots \sqcup \Delta_{-1}^{\prime} \sqcup \Xi_{-1}^{\prime \prime} \sqcup \Delta_{-1}^{\prime \prime} \sqcup \Xi_{0}^{\prime} \sqcup \Delta_{0}^{\prime} \sqcup \Xi_{0}^{\prime \prime} \sqcup \Delta_{0}^{\prime \prime} \sqcup \Xi_{1}^{\prime} \sqcup \Delta_{1}^{\prime} \sqcup \Xi_{1}^{\prime \prime} \sqcup \Delta_{1}^{\prime \prime} \sqcup \cdots
$$

такое, что $\left|\Xi_{j}^{\prime}\right|=\left|\Xi_{j}^{\prime \prime}\right|=8 \varepsilon$ и $I^{\prime}(t)>0$ при $t \in \Xi_{j}^{\prime} \cup \Delta_{j}^{\prime} \cup \Xi_{j}^{\prime \prime}$. Это возможно, например, при

$$
8 \varepsilon<\frac{m_{+}}{m^{\prime}},
$$

где, напомним, $m^{\prime}=\max I^{\prime \prime}(t)$. Теперь фиксируем $\varepsilon, E, K^{\prime}, K^{\prime \prime}$ такие, что выполнены условия предложения 9.5, предыдущее условие, а также

$$
K^{\prime \prime}>300 \frac{1-|\Delta|}{|\Delta|} \frac{m_{-}}{m_{+}} K^{\prime} .
$$

Очевидно, при достаточно малом $\varepsilon$ этого всегда можно добиться.

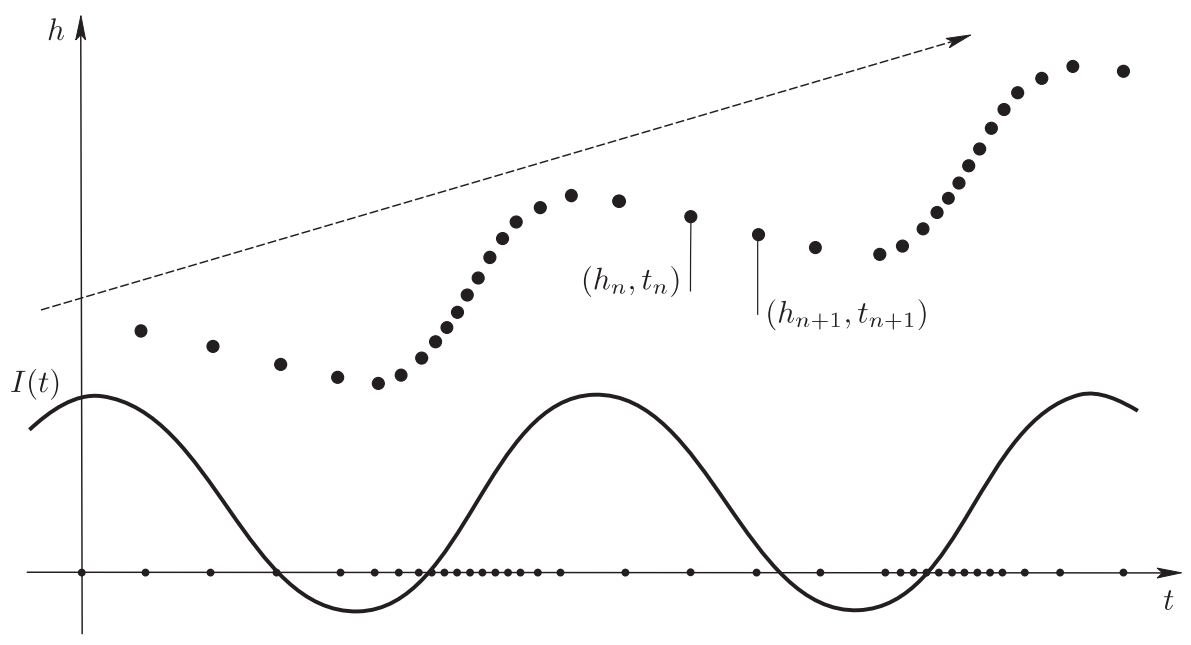

Рис. 9.3. Быстрая траектория Мезера и интеграл Пуанкаре-Мельникова

Теперь рассмотрим отображение $\mathscr{S}_{\mathscr{E}}$. Фиксируем $t_{0}=0, h_{0}=2 E$. Пользуясь предложением 9.5 и леммой 9.7 , построим траекторию такую, что

$$
\begin{aligned}
& t_{n} \in \Delta_{j}^{\prime} \Rightarrow \begin{cases}\bar{x}_{n+1}-\bar{x}_{n}=K^{\prime}+1 & \text { при } n \geqslant 0, \\
\bar{x}_{n}-\bar{x}_{n-1}=K^{\prime}+1 & \text { при } n \leqslant 0,\end{cases} \\
& t_{n} \in \Delta_{j}^{\prime \prime} \Rightarrow \begin{cases}\bar{x}_{n+1}-\bar{x}_{n}=K^{\prime \prime}-1 & \text { при } n \geqslant 0, \\
\bar{x}_{n}-\bar{x}_{n-1}=K^{\prime \prime}-1 & \text { при } n \leqslant 0 .\end{cases}
\end{aligned}
$$

Оценим прирост "энергии" $\Delta h_{j}$ "за время 1": с момента последнего попадания в $\Xi_{j-1}$ до первого попадания в $\Xi_{j+1}$. Обозначим $h_{+}, h_{-}-$максимум и минимум $h_{n}$ за этот период. Тогда имеем:

$$
1 \leqslant \frac{h_{+}}{h_{-}}<2 .
$$


Обозначим:

$$
\begin{array}{rlrl}
\delta t^{\prime} & =\max _{t_{n} \in \Delta_{j}^{\prime}}\left(t_{n+1}-t_{n}\right), & \delta t^{\prime \prime} & =\min _{t_{n} \in \Delta_{j}^{\prime \prime}}\left(t_{n+1}-t_{n}\right), \\
\delta h^{\prime} & =\min _{t_{n} \in \Delta_{j}^{\prime}}\left(h_{n+1}-h_{n}\right), & \delta h^{\prime \prime}=\min _{t_{n} \in \Delta_{j}^{\prime \prime}}\left(h_{n+1}-h_{n}\right), \\
N^{\prime}=\min _{j} \#\left\{t_{n}: t_{n} \in \Delta_{j}^{\prime}\right\}, & N^{\prime \prime}=\max _{j} \#\left\{t_{n}: t_{n} \in \Delta_{j}^{\prime \prime}\right\} .
\end{array}
$$

Имеем следующие оценки:

$$
\begin{array}{rlrl}
\delta t^{\prime} & <\frac{2 K^{\prime}}{\nu \sqrt{h_{-}}}, & \delta t^{\prime \prime}>\frac{K^{\prime \prime}}{2 \nu \sqrt{h_{-}}}, \\
\delta h^{\prime}>\frac{m_{+}}{2 \sqrt{h_{+}}}, & \delta h^{\prime \prime}>-\frac{3 m_{-}}{2 \sqrt{h_{-}}}, \\
N^{\prime}>\frac{|\Delta| \nu \sqrt{h_{-}}}{4 K^{\prime}}, & N^{\prime \prime}<\frac{4(1-|\Delta|) \nu \sqrt{h_{+}}}{K^{\prime \prime}} .
\end{array}
$$

Тогда

$$
\begin{aligned}
\Delta h_{j} & >\frac{|\Delta| \nu \sqrt{h_{-}}}{4 K^{\prime}} \frac{m_{+}}{2 \sqrt{h_{+}}}-\frac{4(1-|\Delta|) \nu \sqrt{h_{+}}}{K^{\prime}} \frac{3 m_{-}}{2 \sqrt{h_{-}}} \\
& >\frac{|\Delta| \nu m_{+}}{16 K^{\prime}}-\frac{9(1-|\Delta|) \nu m_{-}}{K^{\prime \prime}}>\frac{|\Delta| \nu m_{+}}{32 K^{\prime}}=A^{-} .
\end{aligned}
$$

Из (9.13) следует, что $A^{-}>0$. Теорема 9.1 доказана.

\section{Глава 10. Траектории в окрестности асимптотических многообразий в априори неустойчивых системах}

В этой главе мы используем многомерное сепаратрисное отображение Заславского (глава 6) для построения траекторий в априори неустойчивых гамильтоновых системах, близких к интегрируемым. Основным результатом главы является лемма 10.4, выступающая главным рабочим инструментом в главе 11 для построения диффузионных траекторий.

\section{$\S 1$. Уравнения Рауса}

Начнем с равенств (6.8), задающих отображение $\mathscr{S}_{\varepsilon}$. Рассмотрим каноническую замену переменных $(\eta, \xi, \tau, h) \mapsto(\rho, \zeta, \tau, r)$,

$$
\begin{gathered}
\zeta=\xi+\nu(\eta) \tau, \quad \rho=\varepsilon^{-1} \eta, \quad r=\varepsilon^{-1}(h-E(\eta)), \\
d \eta \wedge d \xi+d h \wedge d \tau=\varepsilon(d \rho \wedge d \zeta+d r \wedge d \tau) .
\end{gathered}
$$

Будем считать, что

$$
c^{-1} \varepsilon^{1 / 8}|\ln \varepsilon|<|\widehat{w}|<c \varepsilon^{-1 / 8}, \quad \widehat{w}\left(\rho_{+}, r_{+}, \zeta\right)=r_{+}-\mathbf{H}\left(\varepsilon \rho_{+}, \zeta\right)
$$


(ср. (6.6), (6.7)). В новых координатах $\mathscr{S}_{\varepsilon}$ (формулы (6.8)) выглядит следующим образом: $\mathscr{S}_{\mathscr{\varepsilon}}\left(\zeta, \rho, \tau, r, s ; t_{+}\right)=\left(\zeta_{+}, \rho_{+}, \tau_{+}, r_{+}, s_{+}\right)$,

$$
\begin{aligned}
\rho_{+} & =\rho-\widehat{\Theta}_{\zeta}^{s}\left(\varepsilon \rho_{+}, \zeta, \tau\right)+\frac{\mathbf{H}_{\zeta}\left(\varepsilon \rho_{+}, \zeta\right)}{\lambda} \ln \left|\frac{\varepsilon \kappa^{s} \widehat{w}}{\lambda}\right|+\widehat{\mathbf{O}}_{2}, \\
\zeta_{+} & =\zeta+\nu\left(\varepsilon \rho_{+}\right) t_{+}-\frac{\mathbf{H}_{\rho_{+}}\left(\varepsilon \rho_{+}, \zeta\right)}{\lambda} \ln \left|\frac{\varepsilon \kappa^{s} \widehat{w}}{\lambda}\right|+\widehat{\mathbf{O}}_{1}, \\
r_{+} & =r-\widehat{\Theta}_{\tau}^{s}\left(\varepsilon \rho_{+}, \zeta, \tau\right)+\widehat{\mathbf{O}}_{2}, \\
\tau_{+} & =\tau+t_{+}+\frac{1}{\lambda} \ln \left|\frac{\varepsilon \kappa^{s} \widehat{w}}{\lambda}\right|+\widehat{\mathbf{O}}_{1}, \\
s_{+} & =s \operatorname{sign}(\widehat{w}) .
\end{aligned}
$$

Потенциалы

$$
\widehat{\Theta}^{s}\left(\eta_{+}, \zeta, \tau\right)=\Theta^{s}\left(\eta_{+}, \zeta-\nu\left(\eta_{+}\right) \tau, \tau\right)
$$

периодичны по $\zeta$ и квазипериодичны по $\tau$,

$$
\widehat{\mathbf{O}}_{1}=O^{\bullet}\left(\varepsilon^{7 / 8}\right) \ln ^{2} \varepsilon, \quad \widehat{\mathbf{O}}_{2}=O^{\bullet}\left(\varepsilon^{1 / 8}\right) \ln ^{2} \varepsilon,
$$

где $O^{\bullet}=O^{\left(\varepsilon^{-3 / 4}\right)}$ (см. обозначение $\left.(6.5)\right)$ с особыми переменными $\rho_{+}$.

Производящая функция отображения (10.3) имеет вид

$$
\begin{gathered}
\mathscr{W}=\left\langle\rho_{+}, \zeta\right\rangle+r_{+} \tau+\frac{\widehat{w}}{\lambda} \ln \left|\frac{\varepsilon \kappa^{s} \widehat{w}}{\lambda e}\right|+\widehat{\Theta}^{s}\left(\varepsilon \rho_{+}, \zeta, \tau\right)+\widetilde{\mathscr{W}} \\
\widetilde{\mathscr{W}}\left(\rho_{+}, \zeta, r_{+}, \tau, s, t_{+}, \varepsilon\right)=O^{\bullet}\left(\varepsilon^{1 / 8}\right) \ln ^{2} \varepsilon \\
\rho d \zeta+r d \tau+\zeta_{+} d \rho_{+}+\left(\tau_{+}-t_{+}\right) d r_{+}=d \mathscr{W} .
\end{gathered}
$$

Полагая $\vartheta_{+}=\operatorname{sign}(\widehat{w})$, рассмотрим другую производящую функцию:

$$
\rho d \zeta+r d \tau+\zeta_{+} d \rho_{+}-r_{+} d \tau_{+}=d \mathscr{R}, \quad \mathscr{R}=\mathscr{R}\left(\rho_{+}, \zeta, \tau_{+}, \tau, \vartheta_{+}, s, t_{+}, \varepsilon\right) .
$$

Так как $\mathscr{R}=\mathscr{W}-r_{+}\left(\tau_{+}-t_{+}\right)$, имеем

$$
\begin{aligned}
& \mathscr{R}=\left\langle\rho_{+}, \zeta+\nu\left(\varepsilon \rho_{+}\right) t_{+}\right\rangle-\frac{\vartheta_{+}}{\varepsilon \kappa^{s}} e^{\lambda\left(\tau_{+}-\tau-t_{+}\right)} \\
& \quad-\left(\tau_{+}-\tau-t_{+}\right) \mathbf{H}\left(\varepsilon \rho_{+}, \zeta\right)+\widehat{\Theta}^{s}\left(\varepsilon \rho_{+}, \zeta, \tau\right)+\widetilde{\mathscr{R}}, \\
& \widetilde{\mathscr{R}}\left(\rho_{+}, \zeta, \tau_{+}, \tau, \vartheta_{+}, s, \varepsilon, t_{+}\right)=O^{\bullet}\left(\varepsilon^{1 / 8}\right) \ln ^{2} \varepsilon .
\end{aligned}
$$

Из равенства $r_{+}=-\mathscr{R}_{\tau_{+}}$и соотношений (10.2) вытекает, что

$$
\widehat{w}=\frac{\lambda \vartheta_{+}}{\varepsilon \kappa^{s}} e^{\lambda\left(\tau_{+}-\tau-t_{+}\right)}+O^{\bullet}\left(\varepsilon^{1 / 8}\right) \ln ^{2} \varepsilon
$$

Далее считаем, что $|\tau|<1,\left|\tau_{+}\right|<1$ и при больших положительных $\bar{K}, K_{0}$

$$
\frac{1}{9} \ln \varepsilon \leqslant-\bar{K}-K_{0} \leqslant-\lambda t_{+}-\ln \varepsilon \leqslant-K_{0} .
$$

При малых $\varepsilon>0$ ввиду (10.5) неравенства (10.6) сильнее, чем (10.2). 
Теперь траекториями сепаратрисного отображения мы будем считать последовательности вида

$$
\left(\rho_{k}, \zeta_{k}, \tau_{k}, \vartheta_{k}, s_{k}, t_{k}\right), \quad t_{k} \in \mathbb{N}, \quad k \in \mathbb{Z}
$$

Равенства (10.3) заменяются на следующие:

$$
\begin{aligned}
\rho_{+} & =\rho-\widehat{\Theta}_{\zeta}^{s}\left(\varepsilon \rho_{+}, \zeta, \tau\right)+\left(\tau_{+}-\tau-t_{+}\right) \mathbf{H}_{\zeta}\left(\varepsilon \rho_{+}, \zeta\right)+\widehat{\mathbf{O}}_{2}, \\
\zeta_{+} & =\zeta+\nu\left(\varepsilon \rho_{+}\right) t_{+}-\left(\tau_{+}-\tau-t_{+}\right) \mathbf{H}_{\rho}\left(\varepsilon \rho_{+}, \zeta\right)+\widehat{\mathbf{O}}_{1}, \\
\widehat{\Theta}_{\tau}^{s}\left(\varepsilon \rho_{+}, \zeta, \tau\right) & =\frac{\lambda\left(\varepsilon \rho_{+}\right)}{\varepsilon}\left(\frac{\vartheta}{\kappa^{s-}} e^{\lambda\left(\varepsilon \rho_{+}\right)\left(\tau-\tau_{-}-t\right)}-\frac{\vartheta_{+}}{\kappa^{s}} e^{\lambda\left(\varepsilon \rho_{+}\right)\left(\tau_{+}-\tau-t_{+}\right)}\right)+\widehat{\mathbf{O}}_{2}, \\
s_{+} & =s \vartheta_{+} .
\end{aligned}
$$

Здесь мы опускаем индексы $k$, пишем “+" вместо $k+1$ и “-" вместо $k-1$. Уравнения (10.7) аналогичны уравнениям Рауса из классической механики. Они частично гамильтоновы (относительно $\zeta$ и $\rho$ ) и частично лагранжевы (относительно $\tau$ ).

\section{§ 2. Построение траектории}

Положим

$$
\bar{\nu}=\left(\begin{array}{c}
\nu \\
-1
\end{array}\right) \in \mathbb{R}^{n+1}, \quad \bar{\partial}=\langle\nu, \partial / \partial \xi\rangle-\partial / \partial \tau .
$$

Таким образом, $\bar{\partial}$ - дифференцирование на $\mathbb{T}^{n+1}=\{\xi, \tau\}$ вдоль постоянного векторного поля $\bar{\nu}$. Рассмотрим множества

$$
J_{0}^{s}=\left\{(\eta, \xi, \tau) \in \overline{\mathscr{D}} \times \mathbb{T}^{n+1}: \bar{\partial} \Theta^{s}(\eta, \xi, \tau)=0, \bar{\partial}^{2} \Theta^{s}(\eta, \xi, \tau) \neq 0\right\} .
$$

В общей ситуации для любого $\eta=\eta^{0}$ множество $\left.J_{0}^{s}\right|_{\eta=\eta^{0}} \subset \mathbb{T}^{n+1}$ содержит непустой набор гиперповерхностей, трансверсальных векторному полю $\bar{\nu}$.

Множества $J_{0}^{s}$ полезно записать в координатах $(\rho, \zeta, \tau)$. Положим

$$
\mathscr{J}_{0}^{s}=\left\{(\rho, \zeta, \tau) \in \varepsilon^{-1} \overline{\mathscr{D}} \times \mathbb{T}^{n} \times(-1,1): \widehat{\Theta}_{\tau}^{s}(\varepsilon \rho, \zeta, \tau)=0, \widehat{\Theta}_{\tau \tau}^{s}(\varepsilon \rho, \zeta, \tau) \neq 0\right\} .
$$

Пусть $\pi: \frac{1}{\varepsilon} \overline{\mathscr{D}} \times \mathbb{T}^{n} \times(-1,1) \rightarrow D \times \mathbb{T}^{n+1}$,

$$
\pi(\rho, \zeta, \tau \bmod 1)=(\eta, \xi, \tau \bmod 1)=(\varepsilon \rho, \zeta-\nu(\varepsilon \rho) \tau, \tau \bmod 1), \quad-1<\tau<1 .
$$

Тогда $J_{0}^{s}=\pi\left(\mathscr{J}_{0}^{s}\right)$.

Рассмотрим уравнение

$$
\widehat{\Theta}_{\tau}^{s}(\varepsilon \rho, \zeta, \tau)=z, \quad \tau, z \in \mathbb{R} .
$$

Его можно решить относительно $\tau$ при малых значениях $|z|$ около любой точки $\left(\rho_{0}, \zeta_{0}, \tau_{0}\right) \in \mathscr{J}_{0}^{s}$. Решение является гладкой функцией $\Psi^{s, \rho_{0}, \zeta_{0}, \tau_{0}}(\varepsilon \rho, \zeta, z)$ со 
значениями в $(-2,2)$. Положим

$$
\begin{gathered}
\mathscr{J}_{c^{\prime}, c^{\prime \prime}}^{s}=\left\{\left(\rho_{0}, \zeta_{0}, \tau_{0}\right) \in \mathscr{J}_{0}^{s}: \text { функция } \Psi=\Psi^{s, \rho_{0}, \zeta_{0}, \tau_{0}}(\varepsilon \rho, \zeta, z)\right. \text { гладкая } \\
\text { при } \quad \varepsilon^{3 / 4}\left|\rho-\rho_{0}\right|<c^{\prime},\left|\zeta-\zeta_{0}\right|<c^{\prime},|z|<c^{\prime}, \\
\text { причем } \left.\left|\Psi_{\rho}\right|<\varepsilon^{3 / 4} / c^{\prime \prime},\left|\Psi_{\zeta}\right|<1 / c^{\prime \prime},\left|\Psi_{z}\right|<1 / c^{\prime \prime}\right\}, \\
J_{c^{\prime}, c^{\prime \prime}}^{s}=\left\{(\eta, \xi, \tau) \in J_{0}:(\eta, \xi, \tau)=\pi(\rho, \zeta, \tau), \quad(\rho, \zeta, \tau) \in \mathscr{J}_{c^{\prime}, c^{\prime \prime}}^{s}\right\} .
\end{gathered}
$$

Очевидно,

$$
\bigcup_{c^{\prime}>0, c^{\prime \prime}>0} \mathscr{J}_{c^{\prime}, c^{\prime \prime}}^{s}=\mathscr{J}_{0}^{s}, \quad \bigcup_{c^{\prime}>0, c^{\prime \prime}>0} J_{c^{\prime}, c^{\prime \prime}}^{s}=J_{0}^{s} .
$$

Ниже мы фиксируем достаточно малые $c^{\prime}, c^{\prime \prime}$.

Конструкция траекторий сепаратрисного отображения основана на некоторой индуктивной процедуре. Далее используем обозначение $\Omega=(\rho, \zeta, \tau, \vartheta, s, t)$. Конечная последовательность векторов $\Omega$ обозначается $\mathbb{O}$ :

$$
\mathbb{O}=\left(\Omega_{-k}, \ldots, \Omega_{k}\right), \quad \Omega_{j}=\left(\rho_{j}, \zeta_{j}, \tau_{j}, \vartheta_{j}, s_{j}, t_{j}\right), \quad-k \leqslant j \leqslant k,
$$

где всегда предполагается, что $\Omega_{-k}$ не содержит координату $t$, т.е. $\Omega_{-k}=$ $\left(\rho_{-k}, \zeta_{-k}, \tau_{-k}, \vartheta_{-k}, s_{-k}\right)$. Более того, для краткости мы по умолчанию считаем, что верхние и нижние индексы в $\mathbb{O}($ в $\Omega)$ автоматически переносятся на соответствующую последовательность $\Omega$ (на соответствующий вектор $(\rho, \zeta, \tau, \vartheta, s, t)$ ). Например, $\widehat{\mathbb{O}}^{(l)}=\left(\widehat{\Omega}_{-k}^{(l)}, \ldots, \widehat{\Omega}_{k}^{(l)}\right)$ и $\Omega_{1}^{\prime}=\left(\rho_{1}^{\prime}, \zeta_{1}^{\prime}, \tau_{1}^{\prime}, \vartheta_{1}^{\prime}, s_{1}^{\prime}, t_{1}^{\prime}\right)$.

Постоянные, введенные раньше и те, что появятся далее, удовлетворяют ряду неравенств. Эти неравенства собраны в $\S 6$.

Пусть $b_{\rho}, b_{\zeta}, b_{\tau}-$ положительные постоянные. Далее мы считаем, что они удовлетворяют (10.25)-(10.27). Для любых двух последовательностей $\Omega^{\prime}, \Omega^{\prime \prime}$ определим

$\operatorname{dist}\left(\Omega^{\prime}, \Omega^{\prime \prime}\right)=\left\{\begin{array}{l}+\infty, \quad \text { если } s^{\prime} \neq s^{\prime \prime}, \text { или } \vartheta^{\prime} \neq \vartheta^{\prime \prime}, \text { или } t^{\prime} \neq t^{\prime \prime}, \\ \max \left\{b_{\rho}\left|\rho^{\prime}-\rho^{\prime \prime}\right|, b_{\zeta}\left|\zeta^{\prime}-\zeta^{\prime \prime}\right|, b_{\tau}\left|\tau^{\prime}-\tau^{\prime \prime}\right|\right\} \text { в противном случае. }\end{array}\right.$

Здесь $\left|\rho^{\prime}-\rho^{\prime \prime}\right|,\left|\zeta^{\prime}-\zeta^{\prime \prime}\right|$ и $\left|\tau^{\prime}-\tau^{\prime \prime}\right|$ берутся относительно стандартной метрики в $\mathbb{R}^{n}, \mathbb{T}^{n}$ и $\mathbb{R}$.

ОПРЕДЕЛЕНиЕ 10.1. Последовательность $\overline{\mathbb{O}}=\left(\bar{\Omega}_{-k}, \bar{\Omega}_{-k+1}, \ldots, \bar{\Omega}_{k}\right), k \geqslant 0$, называется квазитраекторией, если

$$
\begin{array}{lll}
\text { (i) } & \left(\bar{\rho}_{j}, \bar{\zeta}_{j}, \bar{\tau}_{j}\right) \in \mathscr{J}_{c^{\prime}, c^{\prime \prime}}^{\bar{s}_{j},} & -k \leqslant j \leqslant k, \\
\text { (ii) } & \bar{t}_{j} \in \mathbb{N}, \quad\left|\bar{\tau}_{j}\right|<1, & -k \leqslant j \leqslant k, \\
\text { (iii) } & K_{0} \leqslant \lambda \bar{t}_{j}+\ln \varepsilon \leqslant K_{0}+\bar{K}, & -k<j \leqslant k, \\
\text { (iv) } & \bar{s}_{j+1}=\bar{s}_{j} \bar{\vartheta}_{j+1}, & -k \leqslant j<k .
\end{array}
$$

В частности, $\overline{\mathbb{O}}$ - квазитраектория с $k=0$, если

$$
\overline{\mathbb{O}}=\bar{\Omega}_{0}=\left(\bar{\rho}_{0}, \bar{\zeta}_{0}, \bar{\tau}_{0}, \bar{\vartheta}_{0}, \bar{s}_{0}\right), \quad\left(\bar{\rho}_{0}, \bar{\zeta}_{0}, \bar{\tau}_{0}\right) \in \mathscr{J}_{c^{\prime}, c^{\prime \prime}}^{\bar{s}_{0},}\left|\bar{\tau}_{0}\right|<1
$$


ОПРЕДЕЛЕНИЕ 10.2. Пусть $b \in(0,1)$ - постоянная. Тректория (O) системы (10.7) называется допустимой, если существует квазитраектория (код) $\overline{\mathbb{O}}$ такая, что

$$
\begin{aligned}
& \operatorname{dist}\left(\Omega_{j}, \bar{\Omega}_{j}\right)<b\left(2-b^{1+k-|j|}\right) \quad \text { для любого целого } j \in[-k, k] \\
& \left(\rho_{ \pm k}, \zeta_{ \pm k}, \tau_{ \pm k}\right) \in \mathscr{J}_{c^{\prime}, c^{\prime \prime}}^{s_{ \pm k}}
\end{aligned}
$$

Далее мы считаем, что $2 b<c^{\prime}$. Поэтому условия (10.12) можно заменить на равенства

$$
\tau_{ \pm k}=\Psi^{s_{ \pm k}, \bar{\rho}_{ \pm k}, \bar{\zeta}_{ \pm k}, \bar{\tau}_{ \pm k}}\left(\varepsilon \rho_{ \pm k}, \zeta_{ \pm k}, 0\right) .
$$

ОПРедЕЛЕНИЕ 10.3. Пусть $\mathbb{O}$ - допустимая траектория с кодом $\overline{\mathbb{O}}$. Скажем, что квазитраектория $\left(\Omega_{+}^{*}, \Omega_{+}\right)$совместна с $\mathbb{O}$ справа, если

$$
\begin{gathered}
\rho_{+}^{*}=\bar{\rho}_{k}, \quad \zeta_{+}^{*}=\bar{\zeta}_{k}, \quad \tau_{+}^{*}=\bar{\tau}_{k}, \quad s_{+}^{*}=\bar{s}_{k}, \quad \vartheta_{+}^{*}=\bar{\vartheta}_{k}, \quad s_{+}=s_{k} \vartheta_{+}, \\
\left|\rho_{+}-\rho_{k}+\widehat{\Theta}_{\zeta}^{s_{k}}\left(\varepsilon \rho_{+}, \zeta_{k}, \tau_{k}\right)-\left(\tau_{+}-\tau_{k}-t_{+}\right) \mathbf{H}_{\zeta}\left(\varepsilon \rho_{+}, \zeta_{k}\right)\right|<\frac{b^{2}}{2 b_{\rho}} \\
\left|\zeta_{+}-\zeta_{k}-\nu\left(\varepsilon \rho_{+}\right) t_{+}+\left(\tau_{+}-\tau_{k}-t_{+}\right) \mathbf{H}_{\rho}\left(\varepsilon \rho_{+}, \zeta_{k}\right)\right|<\frac{b^{2}}{2 b_{\zeta}} .
\end{gathered}
$$

Квазитраектория $\left(\Omega_{-}, \Omega_{-}^{*}\right)$ совместна с $\mathbb{O}$ слева, если

$$
\begin{gathered}
\rho_{-}^{*}=\bar{\rho}_{-k}, \quad \zeta_{-}^{*}=\bar{\zeta}_{-k}, \quad \tau_{-}^{*}=\bar{\tau}_{-k}, \quad s_{-}^{*}=\bar{s}_{-k}, \quad \vartheta_{-}^{*}=\bar{\vartheta}_{-k}, \quad s_{-k}=s_{-} \vartheta_{-k}, \\
\left|\rho_{-k}-\rho_{-}+\widehat{\Theta}_{\zeta}^{s_{-}}\left(\varepsilon \rho_{-k}, \zeta_{-}, \tau_{-}\right)-\left(\tau_{-k}-\tau_{-}-\bar{t}\right) \mathbf{H}_{\zeta}\left(\varepsilon \rho_{-k}, \zeta_{-}\right)\right|<\frac{b^{2}}{2 b_{\rho}} \\
\left|\zeta_{-k}-\zeta_{-}-\nu\left(\varepsilon \rho_{-k}\right) \bar{t}+\left(\tau_{-k}-\tau_{-}-\bar{t}\right) \mathbf{H}_{\rho}\left(\varepsilon \rho_{-k}, \zeta_{-}\right)\right|<\frac{b^{2}}{2 b_{\zeta}}
\end{gathered}
$$

Отметим, что в этом определении единственное различие между $\Omega_{+}^{*}$ и $\bar{\Omega}_{k}$ состоит в том, что $\Omega_{+}^{*}$ не содержит координату $t$. Неравенства в условиях $(10.13)$ вместе с условием (i) для $\left(\Omega_{+}^{*}, \Omega_{+}\right)$и условием $s_{+}=s_{k} \vartheta_{+}$означают, что $\Omega_{+}$приближенно является образом точки $\Omega_{k}$ при сепаратрисном отображении (10.7). Аналогичный смысл имеет условие совместности слева.

ЛЕмма 10.4 (лемма о присоединении, [66]). Пусть $\mathbb{O}^{0}=\left(\Omega_{-k}^{0}, \ldots, \Omega_{k}^{0}\right)-$ допустимая траектория с кодом $\overline{\mathbb{O}}$. Пусть также квазитраектории $\left(\Omega_{-}, \Omega_{-}^{*}\right)$, $\left(\Omega_{+}^{*}, \Omega_{+}\right)$совместны с $\mathbb{O}^{0}$ слева и справа соответственно. Тогда существует допустимая траектория $\widehat{\mathbb{O}}=\left(\widehat{\Omega}_{-k-1}, \ldots, \widehat{\Omega}_{k+1}\right)$ скодом

$$
\begin{aligned}
& \overline{\mathbb{O}}^{*}=\left(\bar{\Omega}_{-k-1}^{*}, \bar{\Omega}_{-k}^{*}, \ldots, \bar{\Omega}_{k}^{*}, \bar{\Omega}_{k+1}^{*}\right), \\
& \bar{\Omega}_{-k-1}^{*}=\Omega_{-}, \quad \bar{\Omega}_{-k}^{*}=\Omega_{-}^{*}, \quad \bar{\Omega}_{-k+1}^{*}=\bar{\Omega}_{-k+1}, \quad \ldots, \quad \bar{\Omega}_{k}^{*}=\bar{\Omega}_{k}, \quad \bar{\Omega}_{k+1}^{*}=\Omega_{+} .
\end{aligned}
$$

Итак, мы можем начать с некоторой допустимой траектории с $k=0$. Если существуют левая и правая совместные квазитраектории, то с помощью леммы 10.4 можно продолжить допустимую траекторию, затем продолжить еще и т.д. Таким образом можно построить широкий класс траекторий, следующих предписанным кодам. 
$\mathrm{K}$ сожалению, в отличие от обычной ситуации, имеющей место в символической динамике, нельзя выбрать бесконечный (или длинный) код заранее. Действительно, согласно определению 10.3 совместные квазитраектории определяются в терминах как кода $\overline{\mathbb{O}}$, так и соответствующей траектории $\mathbb{O}$.

\section{§ 3. Доказательство леммы о присоединении}

Сначала перепишем уравнения (10.7):

$$
\left\{\begin{array}{l}
\rho_{+}=\rho-\widehat{\Theta}_{\zeta}^{s}\left(\varepsilon \rho_{+}, \zeta, \tau\right)+\left(\tau_{+}-\tau-t_{+}\right) \mathbf{H}_{\zeta}\left(\varepsilon \rho_{+}, \zeta\right)+\alpha \\
\zeta_{+}=\zeta+\nu\left(\varepsilon \rho_{+}\right) t_{+}-\left(\tau^{+}-\tau-t_{+}\right) \mathbf{H}_{\rho}\left(\varepsilon \rho_{+}, \zeta\right)+\beta \\
\tau=\Psi^{s, \bar{\rho}, \bar{\zeta}, \bar{\tau}}\left(\varepsilon \rho_{+}, \zeta, \gamma_{0}+\gamma_{1}\right) \\
s_{+}=s \vartheta_{+}
\end{array}\right.
$$

где $(\bar{\rho}, \bar{\zeta}, \bar{\tau}) \in \mathscr{J}_{0}^{s}$ и

$$
\begin{aligned}
\alpha & =\alpha\left(\rho_{+}, \zeta, \tau_{+}, \tau, \vartheta_{+}, s, t_{+}, \varepsilon\right), \\
\beta & =\beta\left(\rho_{+}, \zeta, \tau_{+}, \tau, \vartheta_{+}, s, t_{+}, \varepsilon\right), \\
\gamma_{0} & =\gamma_{0}\left(\tau_{+}, \tau, \tau_{-}, \vartheta_{+}, \vartheta, s, s_{-}, t, t_{+}, \varepsilon\right) \\
& =\frac{\lambda}{\varepsilon}\left(\frac{\vartheta}{\kappa^{s_{-}}} e^{\lambda\left(\tau-\tau_{-}-t\right)}-\frac{\vartheta_{+}}{\kappa^{s}} e^{\lambda\left(\tau_{+}-\tau-t_{+}\right)}\right), \\
\gamma_{1} & =\gamma_{1}\left(\rho_{+}, \rho, \zeta, \zeta_{-}, \tau_{+}, \tau, \tau_{-}, \vartheta_{+}, \vartheta, s, s_{-}, t, t_{+}, \varepsilon\right) .
\end{aligned}
$$

Далее для краткости мы не пишем “дискретные" аргументы $\vartheta, \vartheta_{+}, s_{-}, s, t, t_{+}$, а также $\varepsilon$ у функций $\alpha, \beta, \gamma_{0,1}$.

Пусть $C$ - большая постоянная. Ввиду (10.7) и (10.6)

$$
\begin{gathered}
|\alpha| \leqslant C \mathscr{E}_{2}, \quad|\beta| \leqslant C \mathscr{E}_{1}, \quad\left|\gamma_{0}\right| \leqslant C e^{-K_{0}}, \quad\left|\gamma_{1}\right| \leqslant C \mathscr{E}_{2}, \\
\mathscr{E}_{1}=\varepsilon^{7 / 8} \ln ^{2} \varepsilon, \quad \mathscr{E}_{2}=\varepsilon^{1 / 8} \ln ^{2} \varepsilon .
\end{gathered}
$$

Функции $\alpha, \beta, \gamma_{0}, \gamma_{1}$ удовлетворяют условию Липшица. Имеется в виду следующее. Положим

$$
\begin{aligned}
\alpha^{\prime} & =\alpha\left(\rho_{+}^{\prime}, \zeta^{\prime}, \tau_{+}^{\prime}, \tau^{\prime}\right), \\
\beta^{\prime} & =\beta\left(\rho_{+}^{\prime}, \zeta^{\prime}, \tau_{+}^{\prime}, \tau^{\prime}\right), \\
\gamma_{0}^{\prime} & =\gamma_{0}\left(\tau_{+}^{\prime}, \tau^{\prime}, \tau_{-}^{\prime}\right), \\
\gamma_{1}^{\prime} & =\gamma_{1}\left(\rho_{+}^{\prime}, \rho^{\prime}, \zeta^{\prime}, \zeta_{-}^{\prime}, \tau_{+}^{\prime}, \tau^{\prime}, \tau_{-}^{\prime}\right) .
\end{aligned}
$$

Аналогично определим $\beta^{\prime \prime}, \alpha^{\prime \prime}, \gamma_{0}^{\prime \prime}, \gamma_{1}^{\prime \prime}$ с теми же $\vartheta, \vartheta_{+}, s_{-}, s, t, t_{+}, \varepsilon$. Тогда

$$
\begin{aligned}
\left|\alpha^{\prime}-\alpha^{\prime \prime}\right| \leqslant C \mathscr{E}_{2}\left(\varepsilon^{3 / 4}\left|\rho_{+}^{\prime}-\rho_{+}^{\prime \prime}\right|+\left|\zeta^{\prime}-\zeta^{\prime \prime}\right|+\left|\tau_{+}^{\prime}-\tau_{+}^{\prime \prime}\right|+\left|\tau^{\prime}-\tau^{\prime \prime}\right|\right), \\
\left|\beta^{\prime}-\beta^{\prime \prime}\right| \leqslant C \mathscr{E}_{1}\left(\varepsilon^{3 / 4}\left|\rho_{+}^{\prime}-\rho_{+}^{\prime \prime}\right|+\left|\zeta^{\prime}-\zeta^{\prime \prime}\right|+\left|\tau_{+}^{\prime}-\tau_{+}^{\prime \prime}\right|+\left|\tau^{\prime}-\tau^{\prime \prime}\right|\right), \\
\left|\gamma_{0}^{\prime}-\gamma_{0}^{\prime \prime}\right| \leqslant C e^{-K_{0}}\left(\left|\tau_{+}^{\prime}-\tau_{+}^{\prime \prime}\right|+\left|\tau^{\prime}-\tau^{\prime \prime}\right|+\left|\tau_{-}^{\prime}-\tau_{-}^{\prime \prime}\right|\right), \\
\left|\gamma_{1}^{\prime}-\gamma_{1}^{\prime \prime}\right| \leqslant C \mathscr{E}_{2}\left(\varepsilon^{3 / 4}\left|\rho_{+}^{\prime}-\rho_{+}^{\prime \prime}\right|+\varepsilon^{3 / 4}\left|\rho^{\prime}-\rho^{\prime \prime}\right|+\left|\zeta^{\prime}-\zeta^{\prime \prime}\right|+\left|\zeta_{-}^{\prime}-\zeta_{-}^{\prime \prime}\right|\right. \\
\left.+\left|\tau_{+}^{\prime}-\tau_{+}^{\prime \prime}\right|+\left|\tau^{\prime}-\tau^{\prime \prime}\right|+\left|\tau_{-}^{\prime}-\tau_{-}^{\prime \prime}\right|\right) .
\end{aligned}
$$


Далее $C$ считается липшицевой постоянной для $\Psi^{s, \bar{\rho}, \bar{\zeta}, \bar{\tau}}$ (т.е. $C>1 / c^{\prime \prime}$ ), а также для $\widehat{\Theta}_{\zeta}^{s}, \mathbf{H}_{\zeta}, \mathbf{H}_{\rho}$ и $\nu$ :

$$
\begin{aligned}
\left|\widehat{\Theta}_{\zeta}^{s}\left(\varepsilon \rho^{\prime}, \zeta^{\prime}, \tau^{\prime}\right)-\widehat{\Theta}_{\zeta}^{s}\left(\varepsilon \rho^{\prime \prime}, \zeta^{\prime \prime}, \tau^{\prime \prime}\right)\right| & \leqslant C\left(\varepsilon^{3 / 4}\left|\rho^{\prime}-\rho^{\prime \prime}\right|+\left|\zeta^{\prime}-\zeta^{\prime \prime}\right|+\left|\tau^{\prime}-\tau^{\prime \prime}\right|\right), \\
\left|\mathbf{H}_{\zeta}\left(\varepsilon \rho^{\prime}, \zeta^{\prime}\right)-\mathbf{H}_{\zeta}\left(\varepsilon \rho^{\prime \prime}, \zeta^{\prime \prime}\right)\right| & \leqslant C\left(\varepsilon^{3 / 4}\left|\rho^{\prime}-\rho^{\prime \prime}\right|+\left|\zeta^{\prime}-\zeta^{\prime \prime}\right|\right), \\
\left|\mathbf{H}_{\rho}\left(\varepsilon \rho^{\prime}, \zeta^{\prime}\right)-\mathbf{H}_{\rho}\left(\varepsilon \rho^{\prime \prime}, \zeta^{\prime \prime}\right)\right| & \leqslant C \varepsilon^{3 / 4}\left(\varepsilon^{3 / 4}\left|\rho^{\prime}-\rho^{\prime \prime}\right|+\left|\zeta^{\prime}-\zeta^{\prime \prime}\right|\right), \\
\left|\nu\left(\eta^{\prime}\right)-\nu\left(\eta^{\prime \prime}\right)\right| & \leqslant C\left|\eta^{\prime}-\eta^{\prime \prime}\right| .
\end{aligned}
$$

Кроме того, мы считаем, что

$$
\left|\mathbf{H}_{\zeta}\right|<C, \quad\left|\mathbf{H}_{\rho}\right|<C \varepsilon^{3 / 4} .
$$

Для любой последовательности $\mathbb{O}^{\prime}$, достаточно близкой к

$$
\mathbb{O}^{0+}=\left(\Omega_{-}, \Omega_{-k}^{0}, \ldots, \Omega_{k}^{0}, \Omega_{+}\right)
$$

определим $\mathscr{Q}\left(\mathbb{O}^{\prime}\right)=\mathbb{O}^{\prime \prime}=\left(\Omega_{-k-1}^{\prime \prime}, \ldots, \Omega_{k+1}^{\prime \prime}\right)$ следующим образом. Пусть

$$
\begin{aligned}
\alpha_{j}^{\prime} & =\alpha\left(\rho_{j+1}^{\prime}, \zeta_{j}^{\prime}, \tau_{j+1}^{\prime}, \tau_{j}^{\prime}\right), \quad \beta_{j}^{\prime}=\beta\left(\rho_{j+1}^{\prime}, \zeta_{j}^{\prime}, \tau_{j+1}^{\prime}, \tau_{j}^{\prime}\right), \\
\gamma_{0 j}^{\prime} & =\gamma_{0}\left(\tau_{j+1}^{\prime}, \tau_{j}^{\prime}, \tau_{j-1}^{\prime}\right), \\
\gamma_{1 j}^{\prime} & =\gamma_{1}\left(\rho_{j+1}^{\prime}, \rho_{j}^{\prime}, \zeta_{j}^{\prime}, \zeta_{j-1}^{\prime}, \tau_{j+1}^{\prime}, \tau_{j}^{\prime}, \tau_{j-1}^{\prime}\right) .
\end{aligned}
$$

Полагая для краткости $\Psi^{(j)}=\Psi^{\bar{s}_{j}, \bar{\rho}_{j}, \bar{\zeta}_{j}, \bar{\tau}_{j}}$, мы считаем, что

(i) $\vartheta_{j}^{\prime \prime}=\vartheta_{j}^{\prime}=\bar{\vartheta}_{j}, s_{j}^{\prime \prime}=s_{j}^{\prime}=\bar{s}_{j}, t_{j}^{\prime \prime}=t_{j}^{\prime}=\bar{t}_{j}, j=-k-1, \ldots, k+1$;

(ii) $\zeta_{0}^{\prime \prime}=\zeta_{0}^{\prime}, \rho_{0}^{\prime \prime}=\rho_{0}^{\prime}$;

(iii) при $0 \leqslant j \leqslant k$

$$
\begin{aligned}
\rho_{j+1}^{\prime \prime} & =\rho_{j}^{\prime}-\widehat{\Theta}_{\zeta}^{s_{j}}\left(\rho_{j+1}^{\prime}, \zeta_{j}^{\prime}, \tau_{j}^{\prime}\right)+\left(\tau_{j+1}^{\prime}-\tau_{j}^{\prime}-\bar{t}_{j+1}\right) \mathbf{H}_{\zeta}\left(\varepsilon \rho_{j+1}^{\prime}, \zeta_{j}^{\prime}\right)+\alpha_{j}^{\prime}, \\
\zeta_{j+1}^{\prime \prime} & =\zeta_{j}^{\prime}+\nu\left(\varepsilon \rho_{j+1}^{\prime}\right) \bar{t}_{j+1}-\left(\tau_{j+1}^{\prime}-\tau_{j}^{\prime}-\bar{t}_{j+1}\right) \mathbf{H}_{\rho}\left(\varepsilon \rho_{j+1}^{\prime}, \zeta_{j}^{\prime}\right)+\beta_{j}^{\prime}
\end{aligned}
$$

(iv) при $-k-1 \leqslant j \leqslant-1$

$$
\begin{aligned}
& \rho_{j}^{\prime \prime}=\rho_{j+1}^{\prime}+\widehat{\Theta}_{\zeta}^{s_{j}}\left(\rho_{j+1}^{\prime}, \zeta_{j}^{\prime}, \tau_{j}^{\prime}\right)-\left(\tau_{j+1}^{\prime}-\tau_{j}^{\prime}-\bar{t}_{j+1}\right) \mathbf{H}_{\zeta}\left(\varepsilon \rho_{j+1}^{\prime}, \zeta_{j}^{\prime}\right)-\alpha_{j}^{\prime}, \\
& \zeta_{j}^{\prime \prime}=\zeta_{j+1}^{\prime}-\nu\left(\varepsilon \rho_{j+1}^{\prime}\right) \bar{t}_{j+1}+\left(\tau_{j+1}^{\prime}-\tau_{j}^{\prime}-\bar{t}_{j+1}\right) \mathbf{H}_{\rho}\left(\varepsilon \rho_{j+1}^{\prime}, \zeta_{j}^{\prime}\right)-\beta_{j}^{\prime}
\end{aligned}
$$

(v) при $-k \leqslant j \leqslant k$

$$
\tau_{j}^{\prime \prime}=\Psi^{(j)}\left(\varepsilon \rho_{j+1}^{\prime}, \zeta_{j}^{\prime}, \gamma_{0 j}^{\prime}+\gamma_{1 j}^{\prime}\right) ;
$$

(vi) $\tau_{-k-1}^{\prime \prime}=\Psi^{(-k-1)}\left(\varepsilon \rho_{-k-1}^{\prime}, \zeta_{-k-1}^{\prime}, 0\right), \tau_{k+1}^{\prime \prime}=\Psi^{(k+1)}\left(\varepsilon \rho_{k+1}^{\prime}, \zeta_{k+1}^{\prime}, 0\right)$.

Согласно этому определению любая неподвижная точка оператора $\mathscr{Q}$ является траекторией системы (10.14). Такую траекторию можно считать решением с начальными условиями, заданными для $\rho$ и $\zeta$, и с граничными условиями, заданными для $\tau$.

Определим множество

$$
\begin{gathered}
\mathscr{P}^{s}=\left\{\mathbb{O}=\left(\Omega_{-k-1}, \ldots, \Omega_{k+1}\right): \operatorname{dist}\left(\Omega_{j}, \Omega_{j}^{0+}\right)<\infty,|j| \leqslant k+1\right. \\
\text { и } \left.\quad \Omega_{l}=\Omega_{l}^{0+},|l| \leqslant s\right\} .
\end{gathered}
$$


Имеем очевидные соотношения:

$$
\mathbb{O}^{0+} \in \mathscr{P}^{k} \subset \mathscr{P}^{k-1} \subset \cdots \subset \mathscr{P}^{0} \subset \mathscr{P}^{-1}=\mathscr{P}^{-2}=\cdots .
$$

В соответствии с определением $\mathbb{O}^{0+}$ и $\mathscr{Q}$

если $\mathbb{O} \in \mathscr{P}^{s}, \quad s \leqslant k, \quad$ и $\mathscr{Q}(\mathbb{O})$ определен, то $\mathscr{Q}(\mathbb{O}) \in \mathscr{P}^{s-1}$.

Положим $\mathscr{Q}^{l}\left(\mathbb{O}^{0+}\right)=\mathbb{O}^{(l)}$. В частности, $\mathbb{O}^{(0)}=\mathbb{O}^{0+}$.

ЛЕмма 10.5. Предположим, что постоянные $b, C, b_{\rho}, b_{\zeta}, b_{\tau}, K_{0}, \varepsilon$ удовлетворяют неравенствам из 6 . Тогда для любого $l=0,1,2, \ldots$

$$
\begin{gathered}
\mathbb{O}^{(l)} \in \mathscr{P}^{k-l}, \\
\operatorname{dist}\left(\Omega_{j}^{(l)}, \Omega_{j}^{(l+1)}\right)<b^{4+2 k-2|j|+l}, \quad|j| \geqslant k-l .
\end{gathered}
$$

Согласно (10.19), последовательность $\mathbb{O}^{(l)}, l=0,1,2, \ldots$, сходится. Рассмотрим точку

$$
\widehat{\mathbb{O}}=\lim _{l \rightarrow \infty} \mathbb{O}^{(l)} .
$$

Тогда $\widehat{\mathbb{O}}=\mathscr{Q}(\widehat{\mathbb{O}})$, так что $\widehat{\mathbb{O}}-$ траектория системы $(10.14)$.

СледСтвие 10.6. Так как $\operatorname{dist}\left(\Omega_{j}^{(l)}, \Omega_{j}^{(l+1)}\right)=0$ nрu $|j|<k-l$, имеем:

$$
\operatorname{dist}\left(\widehat{\Omega}_{j}, \Omega_{j}^{0+}\right) \leqslant b^{4+3 k-3|j|}\left(1+b+b^{2}+\cdots\right), \quad|j| \leqslant k+1 .
$$

Лемма 10.7. Траектория (10.20) является допустимой с кодом $\bar{\Omega}^{*}$.

Лемма 10.4 следует из лемм 10.5 и 10.7.

\section{§ 4. Доказательство леммы 10.5}

Включения (10.18) следуют из (10.17). Докажем (10.19), используя индукцию по $l$. Сначала проверим базу индукции. Последовательности $\mathbb{O}^{(0)}$ и $\mathbb{O}^{(1)}$ отличаются только в компонентах $\tau_{ \pm k}, \zeta_{ \pm(k+1)}, \rho_{ \pm(k+1)}$. Далее мы используем свойства постоянных $b, b_{\rho}, b_{\zeta}, b_{\tau}$, собранные в $\S 6$. Ввиду (10.15) имеем:

$$
\begin{gathered}
b_{\rho}\left|\rho_{-k-1}^{(1)}-\rho_{-k-1}^{(0)}\right| \leqslant b_{\rho}\left(\left|\alpha_{-k-1}^{(0)}\right|+\frac{1}{2} b^{2} / b_{\rho}\right) \leqslant b_{\rho}\left(C \mathscr{E}_{2}+\frac{1}{2} b^{2} / b_{\rho}\right) \leqslant b^{2}, \\
b_{\zeta}\left|\zeta_{-k-1}^{(1)}-\zeta_{-k-1}^{(0)}\right| \leqslant b_{\zeta}\left(\left|\beta_{-k-1}^{(0)}\right|+\frac{1}{2} b^{2} / b_{\zeta}\right) \leqslant b_{\zeta}\left(C \mathscr{E}_{1}+\frac{1}{2} b^{2} / b_{\zeta}\right) \leqslant b^{2}, \\
b_{\rho}\left|\rho_{k+1}^{(1)}-\rho_{k+1}^{(0)}\right| \leqslant b_{\rho}\left(\left|\alpha_{k}^{(0)}\right|+\frac{1}{2} b^{2} / b_{\rho}\right) \leqslant b_{\rho}\left(C \mathscr{E}_{2}+\frac{1}{2} b^{2} / b_{\rho}\right) \leqslant b^{2}, \\
b_{\zeta}\left|\zeta_{k+1}^{(1)}-\zeta_{k+1}^{(0)}\right| \leqslant b_{\zeta}\left(\left|\beta_{k}^{(0)}\right|+\frac{1}{2} b^{2} / b_{\zeta}\right) \leqslant b_{\zeta}\left(C \mathscr{E}_{1}+\frac{1}{2} b^{2} / b_{\zeta}\right) \leqslant b^{2} .
\end{gathered}
$$

Наконец,

$$
\begin{aligned}
b_{\tau}\left|\tau_{ \pm k}^{(1)}-\tau_{ \pm k}^{(0)}\right| & =b_{\tau}\left|\Psi^{( \pm k)}\left(\varepsilon \rho_{ \pm k+1}^{(0)}, \zeta_{ \pm k}^{(0)}, \gamma_{0 \pm k}^{(0)}+\gamma_{1 \pm k}^{(0)}\right)-\Psi^{( \pm k)}\left(\varepsilon \rho_{ \pm k}^{(0)}, \zeta_{ \pm k}^{(0)}, 0\right)\right| \\
& \leqslant b_{\tau} C\left(\varepsilon^{3 / 4}\left|\rho_{ \pm k+1}^{(0)}-\rho_{ \pm k}^{(0)}\right|+\left|\gamma_{0 \pm k}^{(0)}\right|+\left|\gamma_{1 \pm k}^{(0)}\right|\right) \\
& \leqslant b_{\tau} C^{2}\left(C \varepsilon^{3 / 4}|\ln \varepsilon|+e^{-K_{0}}+\mathscr{E}_{2}\right) \leqslant b^{4}
\end{aligned}
$$


Теперь предположим, что (10.19) выполнено при некотором $l \geqslant 0$. Полагая $\mathbb{O}^{(l)}=\mathbb{O}^{\prime}, \mathbb{O}^{(l+1)}=\mathbb{O}^{\prime \prime}, \mathbb{O}^{(l+2)}=\mathbb{O}^{\prime \prime \prime}$, мы оценим $\operatorname{dist}\left(\Omega_{j}^{\prime \prime \prime}, \Omega_{j}^{\prime \prime}\right)$.

Согласно соотношениям (ii) из определения $\mathscr{Q}$,

$$
\zeta_{0}^{\prime \prime \prime}=\zeta_{0}^{\prime \prime}=\zeta_{0}^{0+}, \quad \rho_{0}^{\prime \prime \prime}=\rho_{0}^{\prime \prime}=\rho_{0}^{0+}
$$

Используя (iii) и оценки (10.16), при $0 \leqslant j \leqslant k$ получаем, что $b_{\rho}\left|\rho_{j+1}^{\prime \prime \prime}-\rho_{j+1}^{\prime \prime}\right|$ равняется

$$
\begin{aligned}
b_{\rho} \mid \rho_{j}^{\prime \prime}-\widehat{\Theta}_{\zeta}^{\bar{s}_{j}}\left(\varepsilon \rho_{j+1}^{\prime \prime}, \zeta_{j}^{\prime \prime}, \tau_{j}^{\prime \prime}\right)+\left(\tau_{j+1}^{\prime \prime}-\tau_{j}^{\prime \prime}-\bar{t}_{j+1}\right) \mathbf{H}_{\zeta}\left(\varepsilon \rho_{j+1}^{\prime \prime}, \zeta_{j}^{\prime \prime}\right)+\alpha_{j}^{\prime \prime} \\
\quad-\rho_{j}^{\prime}+\widehat{\Theta}_{\zeta}^{\bar{s}_{j}}\left(\varepsilon \rho_{j+1}^{\prime}, \zeta_{j}^{\prime}, \tau_{j}^{\prime}\right)-\left(\tau_{j+1}^{\prime}-\tau_{j}^{\prime}-\bar{t}_{j+1}\right) \mathbf{H}_{\zeta}\left(\varepsilon \rho_{j+1}^{\prime}, \zeta_{j}^{\prime}\right)-\alpha_{j}^{\prime} \mid \\
\leqslant b_{\rho}\left|\rho_{j}^{\prime \prime}-\rho_{j}^{\prime}\right|+b_{\rho} C\left(\varepsilon^{3 / 4}\left|\rho_{j+1}^{\prime \prime}-\rho_{j+1}^{\prime}\right|+\left|\zeta_{j}^{\prime \prime}-\zeta_{j}^{\prime}\right|+\left|\tau_{j}^{\prime \prime}-\tau_{j}^{\prime}\right|\right) \\
\quad+b_{\rho} C|\ln \varepsilon|\left(\varepsilon^{3 / 4}\left|\rho_{j+1}^{\prime \prime}-\rho_{j+1}^{\prime}\right|+\left|\zeta_{j}^{\prime \prime}-\zeta_{j}^{\prime}\right|\right) \\
\quad+b_{\rho} C\left(\left|\tau_{j+1}^{\prime \prime}-\tau_{j+1}^{\prime}\right|+\left|\tau_{j}^{\prime \prime}-\tau_{j}^{\prime}\right|\right) \\
\quad+b_{\rho} C \mathscr{E}_{2}\left(\varepsilon^{3 / 4}\left|\rho_{j+1}^{\prime \prime}-\rho_{j+1}^{\prime}\right|+\left|\zeta_{j}^{\prime \prime}-\zeta_{j}^{\prime}\right|+\left|\tau_{j+1}^{\prime \prime}-\tau_{j+1}^{\prime}\right|+\left|\tau_{j}^{\prime \prime}-\tau_{j}^{\prime}\right|\right) \\
<b^{4+2 k-2 j+l}\left[1+b_{\rho} C\left(\frac{\varepsilon^{3 / 4}}{b_{\rho} b^{2}}+\frac{1}{b_{\zeta}}+\frac{1}{b_{\tau}}\right)\right. \\
\quad+b_{\rho} C\left(\frac{\varepsilon^{3 / 4}|\ln \varepsilon|}{b_{\rho} b^{2}}+\frac{|\ln \varepsilon|}{b_{\zeta}}+\frac{1}{b_{\tau} b^{2}}+\frac{1}{b_{\tau}}\right) \\
\left.\quad+b_{\rho} C \mathscr{E}_{2}\left(\frac{\varepsilon^{3 / 4}}{b_{\rho} b^{2}}+\frac{1}{b_{\zeta}}+\frac{1}{b_{\tau} b^{2}}+\frac{1}{b_{\tau}}\right)\right] \\
<b^{4+2 k-2 j+l}\left[1+\frac{3 C \varepsilon^{3 / 4}|\ln \varepsilon|}{b^{2}}+3 \frac{b_{\rho}}{b_{\zeta}} C|\ln \varepsilon|+5 \frac{b_{\rho}}{b_{\tau}} \frac{C}{b^{2}}\right] \\
\leqslant \\
b^{4+2 k-2(j+1)+l+1} .
\end{aligned}
$$

Величина $b_{\zeta}\left|\zeta_{j+1}^{\prime \prime \prime}-\zeta_{j+1}^{\prime \prime}\right|$ равна

$$
\begin{aligned}
b_{\zeta} \mid \zeta_{j}^{\prime \prime}+ & \nu\left(\varepsilon \rho_{j+1}^{\prime}\right) \bar{t}_{j+1}-\left(\tau_{j+1}^{\prime \prime}-\tau_{j}^{\prime \prime}-\bar{t}_{j+1}\right) \mathbf{H}_{\rho}\left(\varepsilon \rho_{j+1}^{\prime \prime}, \zeta_{j}^{\prime \prime}\right)+\beta_{j}^{\prime \prime} \\
& \quad-\zeta_{j}^{\prime}-\nu\left(\varepsilon \rho_{j+1}^{\prime \prime}\right) \bar{t}_{j+1}+\left(\tau_{j+1}^{\prime}-\tau_{j}^{\prime}-\bar{t}_{j+1}\right) \mathbf{H}_{\rho}\left(\varepsilon \rho_{j+1}^{\prime}, \zeta_{j}^{\prime}\right)-\beta_{j}^{\prime} \mid \\
\leqslant & b_{\zeta}\left|\zeta_{j}^{\prime \prime}-\zeta_{j}^{\prime}\right|+b_{\zeta} C \varepsilon|\ln \varepsilon|\left|\rho_{j+1}^{\prime \prime}-\rho_{j+1}^{\prime}\right| \\
& +b_{\zeta} C \varepsilon^{3 / 4}|\ln \varepsilon|\left(\varepsilon^{3 / 4}\left|\rho_{j+1}^{\prime \prime}-\rho_{j+1}^{\prime}\right|+\left|\zeta_{j}^{\prime \prime}-\zeta_{j}^{\prime}\right|\right) \\
& +b_{\zeta} C \varepsilon^{3 / 4}\left(\left|\tau_{j+1}^{\prime \prime}-\tau_{j+1}^{\prime}\right|+\left|\tau_{j}^{\prime \prime}-\tau_{j}^{\prime}\right|\right) \\
& +b_{\zeta} C \mathscr{E}_{1}\left(\varepsilon^{3 / 4}\left|\rho_{j+1}^{\prime \prime}-\rho_{j+1}^{\prime}\right|+\left|\zeta_{j}^{\prime \prime}-\zeta_{j}^{\prime}\right|+\left|\tau_{j+1}^{\prime \prime}-\tau_{j+1}^{\prime}\right|+\left|\tau_{j}^{\prime \prime}-\tau_{j}^{\prime}\right|\right) \\
< & b^{4+2 k-2 j+l}\left[1+b_{\zeta} \frac{C \varepsilon|\ln \varepsilon|}{b_{\rho} b^{2}}+b_{\zeta} C \varepsilon^{3 / 4}\left(\frac{\varepsilon^{3 / 4}|\ln \varepsilon|}{b_{\rho} b^{2}}+\frac{|\ln \varepsilon|}{b_{\zeta}}+\frac{1}{b_{\tau} b^{2}}+\frac{1}{b_{\tau}}\right)\right. \\
& \left.\quad+b_{\zeta} C \mathscr{E}_{1}\left(\frac{\varepsilon^{3 / 4}}{b_{\rho} b^{2}}+\frac{1}{b_{\zeta}}+\frac{1}{b_{\tau} b^{2}}+\frac{1}{b_{\tau}}\right)\right] \\
\leqslant & b^{4+2 k-2 j+l}\left[1+2 \frac{b_{\zeta}}{b_{\rho}} \frac{C \varepsilon|\ln \varepsilon|}{b^{2}}+3 \varepsilon^{3 / 4} C|\ln \varepsilon|+4 \frac{b_{\zeta}}{b_{\tau}} C \varepsilon^{3 / 4}\right] \\
\leqslant & b^{4+2 k-2(j+1)+l+1} .
\end{aligned}
$$


При $k \leqslant j \leqslant-1$ мы используем (iv). Аналогичные вычисления дают оценки

$$
b_{\rho}\left|\rho_{j}^{\prime \prime \prime}-\rho_{j}^{\prime \prime}\right| \leqslant b^{4+2 k+2 j+l+1}, \quad b_{\zeta}\left|\zeta_{j}^{\prime \prime \prime}-\zeta_{j}^{\prime \prime}\right| \leqslant b^{4+2 k+2 j+l+1} .
$$

Теперь обратимся к переменной $\tau$. При $-k \leqslant j \leqslant k$ величина $b_{\tau}\left|\tau_{j}^{\prime \prime \prime}-\tau_{j}^{\prime \prime}\right|$ равна

$$
\begin{aligned}
b_{\tau}\left|\Psi^{(j)}\left(\varepsilon \rho_{j+1}^{\prime \prime}, \zeta_{j}^{\prime \prime}, \gamma_{0 j}^{\prime \prime}+\gamma_{1 j}^{\prime \prime}\right)-\Psi^{(j)}\left(\varepsilon \rho_{j+1}^{\prime}, \zeta_{j}^{\prime}, \gamma_{0 j}^{\prime}+\gamma_{1 j}^{\prime}\right)\right| \\
\leqslant b_{\tau} C\left(\varepsilon^{3 / 4}\left|\rho_{j}^{\prime \prime}-\rho_{j}^{\prime}\right|+\left|\zeta_{j+1}^{\prime \prime}-\zeta_{j+1}^{\prime}\right|+\left|\gamma_{0 j}^{\prime \prime}-\gamma_{0 j}^{\prime}\right|+\left|\gamma_{1 j}^{\prime \prime}-\gamma_{1 j}^{\prime}\right|\right) \\
\leqslant b_{\tau} C b^{4+2 k-2|j|+l}\left[\frac{\varepsilon^{3 / 4}}{b_{\rho} b^{2}}+\frac{1}{b_{\zeta}}+C e^{-K_{0}}\left(\frac{1}{b_{\tau} b^{2}}+\frac{1}{b_{\tau}}+\frac{b^{2}}{b_{\tau}}\right)\right. \\
\left.\quad+C \mathscr{E} 2\left(\frac{\varepsilon^{3 / 4}}{b_{\rho} b^{2}}+\frac{\varepsilon^{3 / 4}}{b_{\rho}}+\frac{1}{b_{\zeta}}+\frac{1}{b_{\zeta} b^{2}}+\frac{1}{b_{\tau} b^{2}}+\frac{1}{b_{\tau}}+\frac{b^{2}}{b_{\tau}}\right)\right] \\
\leqslant C b^{4+2 k-2|j|+l}\left[3 \frac{b_{\tau}}{b_{\rho} b^{2}} C \varepsilon^{3 / 4}+3 \frac{b_{\tau}}{b_{\zeta}} C+3 \frac{C^{2}}{b^{2}} e^{-K_{0}}+3 \frac{C^{2} \mathscr{E}_{2}}{b^{2}}\right] \\
\leqslant b^{4+2 k-2|j|+l+1} .
\end{aligned}
$$

Имеем также:

$$
\begin{aligned}
b_{\tau}\left|\tau_{-k-1}^{\prime \prime \prime}-\tau_{-k-1}^{\prime \prime}\right| & =b_{\tau}\left|\Psi^{(-k-1)}\left(\varepsilon \rho_{-k-1}^{\prime \prime}, \zeta_{-k-1}^{\prime \prime}, 0\right)-\Psi^{(-k-1)}\left(\varepsilon \rho_{-k-1}^{\prime}, \zeta_{-k-1}^{\prime}, 0\right)\right| \\
& \leqslant b_{\tau} C\left(\frac{\varepsilon^{3 / 4} b^{2}}{b_{\rho}}+\frac{1}{b_{\zeta}}\right) b^{2+l} \leqslant b^{3+l}, \\
b_{\tau}\left|\tau_{k+1}^{\prime \prime \prime}-\tau_{k+1}^{\prime \prime}\right| & =b_{\tau}\left|\Psi^{(k+1)}\left(\varepsilon \rho_{k+1}^{\prime \prime}, \zeta_{k+1}^{\prime \prime}, 0\right)-\Psi^{(k+1)}\left(\varepsilon \rho_{k+1}^{\prime}, \zeta_{k+1}^{\prime}, 0\right)\right| \\
& \leqslant b_{\tau} C\left(\frac{\varepsilon^{3 / 4}}{b_{\rho} b^{2}}+\frac{1}{b_{\zeta} b^{2}}\right) b^{2+l} \leqslant b^{3+l} .
\end{aligned}
$$

Лемма 10.5 доказана.

\section{§ 5. Доказательство леммы 10.7}

Включения (10.12) с $k+1$ вместо $k$ следуют из определения $\widehat{\mathbb{O}}$. Зафиксируем индекс $j \in[-k, k]$. Так как исходная траектория $\mathbb{O}^{(0)}$ допустима с кодом $\overline{\mathbb{O}}$, имеем:

$$
\operatorname{dist}\left(\Omega_{j}^{0}, \bar{\Omega}_{j}\right)<b\left(2-b^{1+k-|j|}\right)
$$

(см. (10.11)). Чтобы доказать, что $\widehat{\mathbb{O}}$ допустима, следует проверить, что

$$
\begin{aligned}
\operatorname{dist}\left(\widehat{\Omega}_{j}, \bar{\Omega}_{j}\right) & <b\left(2-b^{2+k-|j|}\right), \\
\operatorname{dist}\left(\widehat{\Omega}_{ \pm(k+1)}, \Omega_{+}\right) & <b(2-b) .
\end{aligned}
$$

Ввиду (10.21) и (10.22)

$$
\begin{aligned}
\operatorname{dist}\left(\widehat{\Omega}_{j}, \bar{\Omega}_{j}\right) & \leqslant \operatorname{dist}\left(\widehat{\Omega}_{j}, \Omega_{j}^{0+}\right)+\operatorname{dist}\left(\Omega_{j}^{0+}, \bar{\Omega}_{j}\right) \\
& <b^{4+3 k-3|j|}\left(1+b+b^{2}+\cdots\right)+b\left(2-b^{1+k-|j|}\right),
\end{aligned}
$$

откуда получаем (10.23). Неравенство (10.24) следует из (10.21) $\left.\right|_{|j|=k+1}$. 


\section{§ 6. Соотношения между постоянными}

(a) Соотношения между $\varepsilon, \mathscr{E}_{1,2}, \bar{K}, K_{0}$ :

$$
\varepsilon^{7 / 8} \ln ^{2} \varepsilon=\mathscr{E}_{1}, \quad \varepsilon^{1 / 8} \ln ^{2} \varepsilon=\mathscr{E}_{2}, \quad \bar{K}+K_{0}<\frac{1}{9}|\ln \varepsilon| .
$$

(b) Постоянная $C$ :

$$
\begin{gathered}
C>1, \quad C>1 / c^{\prime \prime}, \quad C>\left|H_{\zeta}\right|, \quad C>\left|H_{\rho}\right|, \\
C \text { - липшицева постоянная для } \widehat{\Theta}, \Psi, \mathbf{H}_{\zeta}, \mathbf{H}_{\rho} .
\end{gathered}
$$

(c) Постоянные $b, b_{\rho}, b_{\zeta}, b_{\tau}$ :

$$
\begin{aligned}
b & <1 / 3, \quad 2 b<c^{\prime}, \\
b_{\rho} & =\frac{b^{5}}{60 C^{3}} e^{K_{0}}>1, \\
b_{\tau} & =\frac{b^{4}}{3 C^{2}} e^{K_{0}}, \\
b_{\zeta} & =\frac{b_{\tau} b}{16 C} \varepsilon^{-3 / 4} .
\end{aligned}
$$

Наконец, выпишем неформальные соотношения между основными постоянными: $b, C, b_{\rho} \sim 1$,

$$
\varepsilon<\mathscr{E}_{1}<\mathscr{E}_{2}<b<1<C, \quad b_{\rho}<b_{\tau}<e^{K_{0}}<b_{\zeta}
$$

\section{Глава 11. Диффузия Арнольда в априори неустойчивых системах}

В этой главе мы докажем теоремы 1.6 и 1.7. Теорема 1.6 выполняется при типичном возмущении $H_{1}$. В $\S 2$ формулируются эти условия типичности. Затем они проверяются для $H_{1}(1.14)$. Там же интервал $\overline{\mathscr{D}}$ представляется в виде объединения трех подмножеств: $\overline{\mathscr{D}}=\mathscr{D}_{N} \cup \mathscr{D}_{C R} \cup \mathscr{D}_{V}$ (нерезонансного, четкого и нечеткого резонансного множеств). Каждое из них является конечным набором интервалов. Динамика существенно различна, когда $y$ (или $\eta$ ) лежит в разных множествах, поэтому приходится разбираться с ними по отдельности. Сначала (§3) мы показываем, как проходить через нерезонансный интервал. Затем (§4) мы имеем дело с четким резонансным интервалом. В $\S 5-\S 8$ мы рассматриваем случай нечеткого интервала. В $§ 10$ содержатся доказательства некоторых технических утверждений.

Далее мы считаем, что выполнены условия $\mathbf{H}_{\mathbf{0}} \mathbf{1}-\mathbf{H}_{\mathbf{0}} \mathbf{4}$. Необходимые сведения из предыдущих параграфов - формулировка результатов (§5 введения), формулы для сепаратрисного отображения (10.7) и лемма 10.4.

\section{§ 1. Предварительные соображения}

ПреДЛОЖеНИЕ 11.1. Предположим, что $H_{1}(y, x, 0,0, t) \in C^{j}\left(\mathscr{D}_{0} \times \mathbb{T}^{2}, \mathbb{R}\right) u$

$$
\nu\left(y^{0}\right)=p / q+\delta, \quad p \in \mathbb{Z}, \quad q \in \mathbb{N}, \quad q<\varepsilon^{-1 / 4} / 2 .
$$


Тогда при достаточно мальх є

$$
\left|\mathbf{H}\left(y^{0}, x+p / q\right)-\mathbf{H}\left(y^{0}, x\right)\right|<C \delta^{j} q^{j} .
$$

СлеДСтвие 11.2. Рассмотрим функиию $\widetilde{\mathbf{H}}\left(y^{0}, x\right)=\frac{1}{q} \sum_{l=1}^{q} \mathbf{H}\left(y^{0}, x+l / q\right)$. Тогда $\widetilde{\mathbf{H}} 1 / q$-периодична по $x$ и

$$
\left|\mathbf{H}\left(y^{0}, x\right)-\widetilde{\mathbf{H}}\left(y^{0}, x\right)\right|<C \delta^{j} q^{j+1} .
$$

Доказательство предложения 11.1 содержится в $§ 10$.

Некоторые вычисления для гамильтониана (1.14). Здесь мы получим явные формулы для $\Theta^{+}$и $\mathbf{H}$ в системе с гамильтонианом (1.14). Так как в этом случае $\nu(y)=y$, получаем:

$$
\begin{aligned}
\mathbf{H}(y, x)=a( & \phi\left(y / \varepsilon^{1 / 4}\right) \cos (2 \pi x)+\phi\left(1 / \varepsilon^{1 / 4}\right) \cos (2 \pi t) \\
& +b \phi\left((y-1) / \varepsilon^{1 / 4}\right) \cos (2 \pi(x-t)) \\
& \left.+b \phi\left((y+1) / \varepsilon^{1 / 4}\right) \cos (2 \pi(x+t))\right) .
\end{aligned}
$$

На невозмущенной сепаратрисе $\gamma^{+}=\left\{(v, u): v^{2} / 2+\Omega^{2} \cos (2 \pi u)=\Omega^{2}, v>0\right\}$ естественная параметризация задается равенством

$$
\cos (2 \pi u(t))=1+\frac{i}{\operatorname{sh}(2 \pi \Omega t)-i}-\frac{i}{\operatorname{sh}(2 \pi \Omega t)+i}, \quad i=\sqrt{-1} .
$$

Функция $H_{*}^{+}(y, \xi, \tau, t)$ равна

$$
\begin{aligned}
& a(\cos (2 \pi u(t))-1)(\cos (2 \pi(\xi+y t))+\cos (2 \pi(t-\tau)) \\
& \quad+b \cos (2 \pi(\xi+\tau+y t-t))+b \cos (2 \pi(\xi-\tau+y t+t))) .
\end{aligned}
$$

В результате прямого вычисления получаем:

$$
\begin{aligned}
\Theta^{+}(y, \xi, \tau)= & \frac{y}{\operatorname{sh} y} \cos (2 \pi \xi)+\frac{1}{\operatorname{sh} y} \cos (2 \pi \tau) \\
& \left.+b \frac{y-1}{\operatorname{sh}(y-1)} \cos (2 \pi(\xi+\tau))+b \frac{y+1}{\operatorname{sh}(y+1)} \cos (2 \pi(\xi-\tau))\right) .
\end{aligned}
$$

Здесь мы воспользовались тем, что $\Omega=\pi / 2$ и $a=\pi^{2} / 8$. Функцию $\frac{q}{\operatorname{sh} q}$ в этом равенстве следует доопределить в $q=0$ по непрерывности.

\section{§ 2. Четкие и нечеткие множества}

В этом параграфе мы сформулируем условия $\mathbf{H}_{\mathbf{1}} \mathbf{1}-\mathbf{H}_{\mathbf{1}} \mathbf{3}$ (условия типичности для возмущения $\left.H_{1}\right)$ и разделим $\mathscr{D}$ на три множества $\mathscr{D}_{N}, \mathscr{D}_{C R}$ и $\mathscr{D}_{V}$. Прохождение траектории через каждое из этих множеств будет изучаться по отдельности. 
Далее мы будем строить траектории, на которых $s \equiv \vartheta \equiv+$. Это несколько ограничивает свободу действий, но упрощает обозначения. В дальнейшем индексы $s$ не пишем.

Пусть $J_{0}$ определено с помощью (10.8). Рассмотрим множества

$$
j_{ \pm}(\eta)=\left\{(\xi, \tau) \in \mathbb{T}^{2}:(\eta, \xi, \tau) \in J_{0}, \pm \Theta_{\xi}(\eta, \xi, \tau)>0\right\} .
$$

Пусть $g^{\eta, t}: \mathbb{T}^{2} \rightarrow \mathbb{T}^{2}-$ фазовый поток векторного поля $\bar{\nu}$

$$
g^{\eta, t}(\xi, \tau)=(\xi, \tau)+\bar{\nu}(\eta) t=(\xi+\nu(\eta) t, \tau-t) .
$$

Рассмотрим отображение $\operatorname{pr}_{\bar{\nu}}: \mathbb{T}^{2} \rightarrow \mathbb{T}_{0}=\left\{(\xi, \tau) \in \mathbb{T}^{2}: \tau=0\right\}$,

$$
\operatorname{pr}_{\bar{\nu}}(\xi, \tau)=g^{\eta,\{\tau\}}(\xi, \tau) \equiv(\xi+\nu(\eta)\{\tau\}, 0),
$$

где $\{\tau\} \in[0,1)$ - дробная часть вещественного числа $\tau$. Это отображение является “проекцией” на окружность $\mathbb{T}_{0}$ вдоль векторного поля $\bar{\nu}(\eta)$. Эта проекция разрывна на $\mathbb{T}_{0}$.

Для любого $0 \leqslant \alpha<1 / 2$ и множества $S \subset \mathbb{T}$ положим

$$
S-\alpha=\{\xi \in S:(\xi-\alpha, \xi+\alpha) \subset S\} .
$$

$\mathbf{H}_{1}$ 1. Существует $c_{*}>0$, не зависящее от $\eta \in \overline{\mathscr{D}}$, такое, что множества $\operatorname{pr}_{\bar{\nu}} j_{ \pm}(\eta)-2 c_{*}$ Henycmb.

ПрЕДЛОЖЕНИЕ 11.3. Множество $C^{j}$-гладких функиий $\Theta(\eta, \xi, \tau), 2 \leqslant j \leqslant \omega$, удовлетворяющих $\mathbf{H}_{\mathbf{1}} \mathbf{1}$, открыто и плотно в топологии $C^{j}$.

Доказательство утверждения 11.3 опустим (см. [66]).

ОПРЕДЕЛЕНИЕ 11.4. Предположим, что выполнено условие $\mathbf{H}_{\mathbf{1}} \mathbf{1}$ и $c_{*}-$ соответствующая постоянная. Назовем $\eta \in \overline{\mathscr{D}} K$-четким, если

$$
\bigcup_{0 \leqslant t \leqslant K-1} g^{\eta, t}\left(\operatorname{pr}_{\bar{\nu}} j_{ \pm}-c_{*}\right)=\mathbb{T}^{2} .
$$

Иначе $\eta$ называется $K$-нечетким.

ПреДЛОЖЕНИЕ 11.5. Пусть $\eta \in \overline{\mathscr{D}} K$-нечеткое $u I \subset \operatorname{pr}_{\bar{\nu}} j_{ \pm}(\eta)-c_{*}-$ интервал длинъ $|I|$. Тогда существуют $p \in \mathbb{Z}, q \in \mathbb{N}$ такие, что

$$
q \leqslant K-1, \quad q<\frac{2}{|I|}, \quad\left|\nu(\eta)-\frac{p}{q}\right|<\frac{1}{q K} .
$$

СлЕДСТВИЕ 11.6. Если выполнено условие $\mathbf{H}_{\mathbf{1}} \mathbf{1}$, mо $q<1 / c_{*}$ в (11.3). Так что, согласно предложению 11.5, нечеткие значения $\eta$ собраны в интервалы вокруг резонансов низких порядков. Количество этих интервалов конечно $u$ не зависит от $\varepsilon$.

Доказательство предложения 11.5 содержится в § 10. Далее

$$
K=\bar{K} / \lambda-4, \quad \bar{K}=|\ln \varepsilon| / 10 .
$$


ОПРЕДЕЛЕНИЕ 11.7. Назовем $\eta \in \overline{\mathscr{D}}$ существенным, если

$$
\frac{|\ln \varepsilon|}{\lambda} \max _{\zeta \in \mathbb{T}}\left|\mathbf{H}_{\zeta}(\eta, \zeta)\right|>b .
$$

Существенные точки $\eta \in \overline{\mathscr{D}}$ образуют интервалы, на которых $\nu(\eta)$ близки к резонансам не очень высоких порядков. Точнее, имеем следующее предложение.

ПредЛОЖЕНИЕ 11.8. Пусть $\eta \in \overline{\mathscr{D}}$ существенно и $H_{1} \quad C^{j}$-гладкий, $j \geqslant 2$. Тогда существуют $k, k_{0} \in \mathbb{Z},|k|<c^{-1}(|\ln \varepsilon| / b)^{1 /(j-1)}$ такие, что

$$
\left|k \nu(\eta)+k_{0}\right| \leqslant \varepsilon^{1 / 4} .
$$

Доказательство предложения 11.8 следует из оценки $\left|H_{1}^{l, l_{0}}\right|<c_{1}^{-1}\left(|l|+\left|l_{0}\right|\right)^{-j}$ для коэффициентов Фурье функции $H_{1}$ и определения функции $\mathbf{H}$.

Пусть $\mathscr{E}$ - множество существенных точек и $\mathscr{D}_{C}-$ множество $K$-четких точек в $\overline{\mathscr{D}}$, где $K$ удовлетворяет (11.4). Назовем

$$
\mathscr{D}_{N}=\mathscr{D}_{C} \backslash \mathscr{E}, \quad \mathscr{D}_{C R}=\mathscr{D}_{C} \cap \mathscr{E}, \quad \mathscr{D}_{V}=\overline{\mathscr{D}} \backslash \mathscr{D}_{C}
$$

нерезонансным, четким резонансным и нечетким резонансным множеством соответственно. Тогда $\overline{\mathscr{D}}=\mathscr{D}_{N} \cup \mathscr{D}_{C R} \cup \mathscr{D}_{V}$. Множества $\mathscr{D}_{N}, \mathscr{D}_{C R}$ и $\mathscr{D}_{V}-$ конечные наборы интервалов.

Рассмотрим резонанс $\eta=\eta^{0}$, порождающий нечеткий интервал $\mathbf{I} \subset \mathscr{D}_{V}(\mathrm{~cm}$. следствие 11.6). Обозначим $\nu_{0}=\nu\left(\eta^{0}\right)=p_{0} / q_{0}$, где $p_{0} \in \mathbb{Z}, q_{0} \in \mathbb{N}, q_{0}<2 / c_{*}$ и дробь $p_{0} / q_{0}$ несократима. Рассмотрим две функции:

$$
\begin{aligned}
& F_{+}\left(\eta^{0}, \zeta\right)=\max _{u \in\left[-q_{0}, 0\right]}\left\{\Theta_{\xi}\left(\eta^{0}, \zeta-\nu_{0} u, u\right):\left(\eta^{0}, \zeta-\nu_{0} u, u\right) \in \bar{J}_{0}\right\}, \\
& F_{-}\left(\eta^{0}, \zeta\right)=\min _{u \in\left[-q_{0}, 0\right]}\left\{\Theta_{\xi}\left(\eta^{0}, \zeta-\nu_{0} u, u\right):\left(\eta^{0}, \zeta-\nu_{0} u, u\right) \in \bar{J}_{0}\right\} .
\end{aligned}
$$

Очевидно, что если $F_{+}$определена в точке $\zeta$, то $F_{-}$тоже определена в $\zeta$ (и наоборот), и, более того, $F_{+}\left(\eta^{0}, \zeta\right) \geqslant F_{-}\left(\eta^{0}, \zeta\right)$. В типичной ситуации $F_{ \pm}$ определены при всех $\zeta$ (см. предложение 11.10 ниже).

Функция $\Theta\left(\eta^{0}, \zeta-\nu_{0} u, u\right) \quad q_{0}$-периодична по $u$. Поэтому $F_{ \pm}$могут быть определены как максимум и минимум по $u \in \mathbb{R}$. Функции $F_{ \pm}\left(\eta^{0}, \zeta\right) 1 / q_{0^{-}}$ периодичны по $\zeta$. Определим усреднение:

$$
\left\langle F_{ \pm}\right\rangle\left(\eta^{0}\right)=q_{0} \int_{0}^{1 / q_{0}} F_{ \pm}\left(\eta^{0}, \zeta\right) d \zeta .
$$

ОПРЕДЕлЕниЕ 11.9. Назовем функцию $f: \mathbb{T} \rightarrow \mathbb{R}$ кусочно гладкой, если существуют конечный набор точек ${ }^{12} \varphi_{1}, \ldots, \varphi_{k} \in \mathbb{T}$ и непрерывные функции $f_{j}:\left[\varphi_{j}, \varphi_{j+1}\right] \rightarrow \mathbb{R}$ такие, что

1) $f_{j}$ гладкие на интервалах $\left(\varphi_{j}, \varphi_{j+1}\right) \subset \mathbb{T}$,

2) $\left.f\right|_{\left(\varphi_{j}, \varphi_{j+1}\right)}=f_{j}$

\footnotetext{
${ }^{12}$ Предполагается, что $\varphi_{1}, \ldots, \varphi_{k}$ упорядочены на окружности, т.е., двигаясь от $\varphi_{j} \mathrm{k} \varphi_{j+1}$ в положительном направлении, мы не встретим других точек $\varphi_{s}\left(\right.$ здесь $\left.\varphi_{k+1}=\varphi_{1}\right)$.
} 
Отметим, что согласно этому определению кусочно гладкая функция может быть разрывной.

ПРЕДЛОЖЕНИЕ 11.10. Для $C^{j}$-типичной $\Theta$

1) $\pm\left\langle F_{ \pm}\right\rangle>0$;

2) $F_{+}=F_{-}$в не более чем конечном числе точек $\zeta_{1}, \ldots, \zeta_{k} \in \mathbb{T}$, где для достаточно малых $|\delta|$ выполнены следующие условия трансверсальности:

$$
F_{+}\left(\eta^{0}, \zeta_{j}+\delta\right)-F_{-}\left(\eta^{0}, \zeta_{j}+\delta\right)>2 c_{F}|\delta|, \quad c_{F}>0, \quad j=1, \ldots, k ;
$$

3) $F_{ \pm}\left(\eta^{0}, \zeta\right)=\Theta_{\xi}\left(\eta^{0}, \zeta-\nu_{0} \gamma_{ \pm}(\zeta), \gamma_{ \pm}(\zeta)\right)$, где для некоторого $c_{F}^{\prime}>0$

$$
\bar{\partial} \Theta\left(\eta^{0}, \zeta-\nu_{0} \gamma_{ \pm}(\zeta), \gamma_{ \pm}(\zeta)\right)=0, \quad\left|\bar{\partial}^{2} \Theta\left(\eta^{0}, \zeta-\nu_{0} \gamma_{ \pm}(\zeta), \gamma_{ \pm}(\zeta)\right)\right| \geqslant 2 c_{F}^{\prime}
$$

4) функции $\gamma_{ \pm}$и $F_{ \pm}$кусочно $C^{j-1}$-гладкие по $\zeta$.

Типичность здесь понимается в том смысле, что множество $\Theta$, удовлетворяющих 1)-4), содержит открытое и плотное в $C^{j}$-топологии подмножество пространства $C^{j}$.

Доказательство предложения 11.10 опустим (см. [66]).

$\mathbf{H}_{1}$ 2. Для любого $\eta^{0}$, порождающего нечеткий интервал, функиия $\Theta\left(\eta^{0}, \zeta, \tau\right)$ типична в смысле предложения 11.10.

$\mathbf{H}_{\mathbf{1}}$ 3. Для любого $\eta^{0}$, порождающего нечеткий интервал, функция $\mathbf{H}\left(\eta^{0}, \zeta\right)$, $\zeta=\zeta \bmod \frac{1}{q_{0}}$, имеет глобальный максимум в единственной точке $\zeta_{*}=\zeta_{*}\left(\eta^{0}\right)$ и этот максимум невырожден.

Условия $\mathbf{H}_{\mathbf{1}} \mathbf{1}-\mathbf{H}_{\mathbf{1}} \mathbf{3}$ и система (1.14). Укажем идею доказательства теоремы 1.7. Нам надо проверить, что функции $\mathbf{H}$ и $\Theta=\Theta^{+}$, построенные в конце $\S 1$, удовлетворяют условиям $\mathbf{H}_{\mathbf{1}} \mathbf{1}-\mathbf{H}_{\mathbf{1}} \mathbf{3}$. Сначала положим $b=0$. Так как $\bar{\partial}=\eta \partial / \partial \xi-\partial / \partial \tau$, имеем:

$$
\begin{aligned}
\bar{\partial} \Theta(\eta, \xi, \tau) & =-2 \pi\left(\frac{\eta^{2}}{\operatorname{sh} \eta} \sin (2 \pi \xi)-\frac{1}{\operatorname{sh} 1} \sin (2 \pi \tau)\right) \\
\bar{\partial}^{2} \Theta(\eta, \xi, \tau) & =-4 \pi^{2}\left(\frac{\eta^{3}}{\operatorname{sh} \eta} \cos (2 \pi \xi)+\frac{1}{\operatorname{sh} 1} \cos (2 \pi \tau)\right) .
\end{aligned}
$$

Для любого $\eta^{0}$ множество $J_{0} \cap\left\{\eta=\eta^{0}\right\}$ состоит из кривых, определенных равенством $\bar{\partial} \Theta=0$, за вычетом конечного множества, где $\bar{\partial}^{2} \Theta=0$. Существует только два исключения: $\eta^{0}= \pm 1$. Например, при $\eta^{0}=1$ приходится удалить кривую $\{\xi+\tau=1 / 2\}$, где выполнены оба равенства. Однако другая кривая $\{\xi=\tau\}$ без двух точек $\xi=\tau= \pm 1 / 4$ лежит в $J_{0} \cap\{\eta=1\}$. При $\eta=-1$ ситуация аналогична.

При $\left|\eta^{2} / \operatorname{sh} \eta\right| \neq 1 / \operatorname{sh} 1$ множество $\{(\xi, \tau): \bar{\partial} \Theta(\eta, \xi, \tau)=0\}$ состоит из двух кривых, проходящих через четыре точки $(\xi, \tau) \in \mathbb{Z}^{2} / 2 \bmod 1$ (две точки на каждой кривой). Обе кривые гомотопны либо $\{\tau=0\}$, либо $\{\xi=0\}$ в зависимости от того, какое из неравенств $\left|\eta^{2} / \operatorname{sh} \eta\right|>1 / \operatorname{sh} 1$ или $\left|\eta^{2} / \operatorname{sh} \eta\right|<1 / \operatorname{sh} 1$ выполнено. 
Так как $\Theta_{\xi}=-(2 \pi \eta / \operatorname{sh} \eta) \sin (2 \pi \xi)$, мы видим, что

$$
j_{ \pm}\left(\eta^{0}\right)=J_{0} \cap\left\{\eta=\eta^{0}\right\} \cap\{\mp \sin (2 \pi \xi)>0\} .
$$

Используя эти наблюдения, легко проверить выполнение условий $\mathbf{H}_{\mathbf{1}} \mathbf{1}$.

Нечеткие интервалы являются окрестностями целых $\eta$. Итак, необходимо проверить $\mathbf{H}_{\mathbf{2}} \mathbf{2}$ только для $\eta= \pm 1$ и $\eta=0$. При $\eta= \pm 1$ соответствующие функции $F_{ \pm}$удовлетворяют предложению 11.10 . В случае $\eta^{0}=0$ имеем:

$$
F_{+}(0, \zeta)=F_{-}(0, \zeta)=\sin \zeta
$$

т.е. имеет место вырожденный случай. Вырождение исчезает при малых $b$.

Условие $\mathbf{H}_{\mathbf{1}} \mathbf{3}$ при $\eta^{0}= \pm 1,0$ легко проверяется при $b \neq 0$.

\section{§ 3. Прохождение через нерезонансный интервал}

Обозначим $\lambda_{\min }=\min _{y \in \mathscr{D}} \lambda(y)$. Согласно $\mathbf{H}_{\mathbf{0}} \mathbf{2}, \lambda_{\min }>0$.

Рассмотрим компоненту связности $\left(a_{1}, a_{2}\right)$ в $\mathscr{D}_{N}$ и допустимую траекторию $\mathbb{O}=\left(\Omega_{-k}, \ldots, \Omega_{k}\right)$, входящую в этот интервал, т.е. $a_{1} \leqslant \varepsilon \rho_{k} \leqslant a_{1}+O(\varepsilon|\ln \varepsilon|)$.

Лемма 11.11. Предположим, что выполнено условие $\mathbf{H}_{\mathbf{1}} \mathbf{1}$. Тогда можно продолжить траекторию до допустимой траектории, покидающей $\left(a_{1}, a_{2}\right)$ через другой конеи: $a_{2} \leqslant \varepsilon \rho_{k^{\prime}} \leqslant a_{2}+O(\varepsilon|\ln \varepsilon|)$ при некотором $k^{\prime}>k$. Более mого,

$$
\sum_{j=k}^{k^{\prime}} t_{j}<\frac{\left(a_{2}-a_{1}\right)|\ln \varepsilon|}{2 b \varepsilon \lambda_{\min }} .
$$

СлЕДСТвиЕ 11.12. Средняя скорость прохождения через $\left(a_{1}, a_{2}\right)$

$$
\frac{\left|\eta_{2}-\eta_{1}\right|}{\sum_{j=k}^{k^{\prime}} t_{j}}=\varepsilon \frac{\left|\rho_{2}-\rho_{1}\right|}{\sum_{j=k}^{k^{\prime}} t_{j}}
$$

имеет порядок $\varepsilon /|\ln \varepsilon|$.

ДоКАЗАТЕЛЬСтво ЛЕммы 11.11. Пусть $\overline{\mathbb{O}}=\left(\bar{\Omega}_{-k}, \ldots, \bar{\Omega}_{k}\right)-$ код траектории $\mathbb{O}$. Мы обсудим продолжение траектории вперед по времени. (Продолжение назад несущественно.)

ЛЕмма 11.13. Предположим, что $\mathbf{H}_{\mathbf{1}} \mathbf{1}$ выполнено и точка $\varepsilon \rho_{k} \quad K$-четкал, где K удовлетворяет (11.4). Тогда существует правая совместная квазитраектория $\left(\bar{\Omega}_{k}, \bar{\Omega}_{k+1}\right)$ такая, что

$$
\widehat{\Theta}_{\zeta}\left(\varepsilon \bar{\rho}_{k+1}, \bar{\zeta}_{k+1}, \bar{\tau}_{k+1}\right)<-7 b .
$$

Теперь лемма 11.11 следует из леммы 10.4. Действительно, новая траектория $\widehat{\mathbb{O}}=\left(\Omega_{-k-1}, \ldots, \Omega_{k+1}\right)$ такова, что $\operatorname{dist}\left(\Omega_{k+1}, \bar{\Omega}_{k+1}\right)<2 b$. В частности, $b_{\rho}\left|\rho_{k+1}-\bar{\rho}_{k+1}\right|<2 b$. Это неравенство сохраняется при продолжении траектории путем индуктивного применения леммы о присоединении. Так как речь идет о нерезонансном интервале, $\left|\left(\bar{\tau}_{k+1}-\tau-\bar{t}_{k+1}\right) \mathbf{H}_{\zeta}\left(\varepsilon \bar{\rho}_{k+1}, \zeta\right)\right|<b$. Отсюда 
следует (см. (10.7)), что $\bar{\rho}_{k+1}-\bar{\rho}_{k}>5 b$. Таким образом, на продолжениях траектории $\mathbb{O}$

$$
\rho_{k+1}-\rho_{k} \geqslant-\left|\rho_{k+1}-\bar{\rho}_{k+1}\right|+\bar{\rho}_{k+1}-\bar{\rho}_{k}-\left|\bar{\rho}_{k}-\rho_{k}\right|>5 b-4 b / b_{\rho}>4 b .
$$

Это означает, что на траектории переменная $\rho$ возрастает, по крайней мере, на $4 b$ на каждом шаге. Каждый шаг требует времени менее, чем $2|\ln \varepsilon| / \lambda_{\min }$, откуда получаем (11.7).

\section{ДоКАЗАТЕЛЬСТвО ЛЕмМЫ 11.13. Определим}

$$
\widetilde{j}_{ \pm}\left(\eta, c, c^{\prime}, c^{\prime \prime}\right)=\left\{(\xi, \tau) \in \mathbb{T}^{2}:(\eta, \xi, \tau) \in J_{c^{\prime}, c^{\prime \prime}}, \pm \Theta_{\xi}(\eta, \xi, \tau) \geqslant c\right\} .
$$

Так как $\bigcup_{c, c^{\prime}, c^{\prime \prime}>0} \widetilde{j}_{ \pm}\left(\eta, c, c^{\prime}, c^{\prime \prime}\right)=j_{ \pm}(\eta)$, то для любого $\eta \in \overline{\mathscr{D}}$ существует $\widetilde{c}>0$ такое, что

$$
\operatorname{pr}_{\bar{\nu}} \widetilde{j}_{ \pm}(\eta, \widetilde{c}, \widetilde{c}, \widetilde{c}) \subset \operatorname{pr}_{\bar{\nu}} j_{ \pm}-c_{*} .
$$

Так как $\overline{\mathscr{D}}$ - компакт, то можно выбрать $\widetilde{c}>0$ не зависящим от $\eta \in \overline{\mathscr{D}}$.

Итак, для любого $K$-четкого $\eta$ и достаточно малых $b, c^{\prime}, c^{\prime \prime}$ имеем:

$$
\bigcup_{0 \leqslant t \leqslant K+1} g^{\eta, t}\left(\operatorname{pr}_{\bar{\nu}} \widetilde{j}_{ \pm}\left(\eta, 8 b, 2 c^{\prime}, 2 c^{\prime \prime}\right)\right)=\mathbb{T}^{2} .
$$

Построим правую совместную квазитраекторию $\left(\bar{\Omega}_{k}, \bar{\Omega}_{k+1}\right)$. Далее для краткости положим $\Omega_{+}=\bar{\Omega}_{k+1}$ и будем иметь дело с первым неравенством (11.8). Согласно определению правой совместной траектории, необходимо найти $\rho_{+}$, $\zeta_{+}, \tau_{+}, t_{+}$такие, что

$$
\begin{aligned}
& \left|\rho_{+}-\rho_{k}+\widehat{\Theta}_{\zeta}\left(\varepsilon \rho_{+}, \zeta_{k}, \tau_{k}\right)-\left(\tau_{+}-\tau_{k}-t_{+}\right) \mathbf{H}_{\zeta}\left(\varepsilon \rho_{+}, \zeta_{k}\right)\right|<\frac{b^{2}}{2 b_{\rho}}, \\
& \left|\zeta_{+}-\zeta_{k}-\nu\left(\varepsilon \rho_{+}\right) t_{+}+\left(\tau_{+}-\tau_{k}-t_{+}\right) \mathbf{H}_{\rho}\left(\varepsilon \rho_{+}, \zeta_{k}\right)\right|<\frac{b^{2}}{2 b_{\zeta}}, \\
& \left(\rho_{+}, \zeta_{+}, \tau_{+}\right) \in \mathscr{J}_{c^{\prime}, c^{\prime \prime}}, \quad \bar{K}, \quad\left|\tau_{+}\right|<1, \quad t_{+} \in \mathbb{N} . \\
& K_{0} \leqslant \lambda t_{+}+\ln \varepsilon \leqslant K_{0}+\bar{K}
\end{aligned}
$$

Более того, из-за условия $\widehat{\Theta}_{\zeta}\left(\varepsilon \rho_{+}, \zeta_{+}, \tau_{+}\right)<-7 b$, вместо (11.13) требуется более сильное условие:

$$
\left(\eta_{+}, \xi_{+}, \tau_{+}\right) \equiv\left(\varepsilon \rho_{+}, \zeta_{+}-\nu\left(\eta_{+}\right) \tau_{+}, \tau_{+}\right) \in \widetilde{j}_{-}\left(\eta_{+}, 7 b, c^{\prime}, c^{\prime \prime}\right)
$$

(1) Сначала найдем $t_{+}$и некоторые вспомогательные $\tau_{*}, \zeta_{*}$. Рассмотрим условие

$$
\left(\varepsilon \rho_{k}, \zeta_{k}+\nu\left(\varepsilon \rho_{k}\right) t,-t\right) \in \widetilde{j}_{-}\left(\eta_{k}, 8 b, 2 c^{\prime}, 2 c^{\prime \prime}\right) .
$$

Согласно (11.10) и (11.4), для любой точки $\left(\xi_{0}, \tau_{0}\right) \in \mathbb{T}^{2}$ любой интервал $I \subset \mathbb{R}$ длины большей или равной $\bar{K} / \lambda-1$ содержит точку $t$ такую, что $\left(\eta_{k}, \xi_{0}+\right.$ $\left.\nu\left(\varepsilon \rho_{k}\right) t, \tau_{0}-t\right)$ лежит в $\widetilde{j}_{-}\left(\eta_{k}, 8 b, 2 c^{\prime}, 2 c^{\prime \prime}\right)$. Таким образом, (11.16) имеет решение

$$
t=\widehat{t}, \quad K_{0}+\lambda / 2 \leqslant \lambda \widehat{t}+\ln \varepsilon \leqslant K_{0}+\bar{K}-\lambda / 2 .
$$


Определим $t_{+}, \tau_{*}, \zeta_{*}$ следующим образом:

$$
t_{+}-\tau_{*}=\widehat{t}, \quad t_{+} \in \mathbb{Z}, \quad \tau_{*} \in(-1 / 2,1 / 2], \quad \zeta_{*}=\zeta_{k}+\nu\left(\varepsilon \rho_{k}\right) t_{+} .
$$

Тогда выполнено (11.14). Из (11.16) вытекает, что

$$
\left(\varepsilon \rho_{k}, \zeta_{*}-\nu\left(\varepsilon \rho_{k}\right) \tau_{*}, \tau_{*}\right) \in \widetilde{j}_{ \pm}\left(\eta_{k}, 8 b, 2 c^{\prime}, 2 c^{\prime \prime}\right) .
$$

(2) Определим $\rho_{+}, \zeta_{+}$:

$$
\begin{aligned}
\rho_{+} & =\rho_{k}-\widehat{\Theta}_{\zeta}\left(\varepsilon \rho_{k}, \zeta_{k}, \tau_{k}\right)+\left(\tau_{*}-\tau_{k}-t_{+}\right) \mathbf{H}_{\zeta}\left(\varepsilon \rho_{k}, \zeta_{k}\right), \\
\zeta_{+} & =\zeta_{k}+\nu\left(\varepsilon \rho_{k}\right) t_{+}-\left(\tau_{*}-\tau_{k}-t_{+}\right) \mathbf{H}_{\rho}\left(\varepsilon \rho_{k}, \zeta_{k}\right) .
\end{aligned}
$$

(3) Найдем $\tau_{+}$из уравнения $\bar{\partial} \Theta\left(\varepsilon \rho_{+}, \zeta_{+}-\nu\left(\varepsilon \rho_{+}\right) \tau_{+}, \tau_{+}\right)=0$, которое можно записать в виде $\widehat{\Theta}_{\tau}\left(\varepsilon \rho_{+}, \zeta_{+}, \tau_{+}\right)=0$. Следовательно,

$$
\tau_{+}=\Psi^{\rho_{k}, \zeta_{*}, \tau_{*}}\left(\varepsilon \rho_{+}, \zeta_{+}, 0\right) .
$$

Так как $\tau_{*}=\Psi^{\rho_{k}, \zeta_{*}, \tau_{*}}\left(\varepsilon \rho_{k}, \zeta_{*}, 0\right),\left|\rho_{+}-\rho_{k}\right|=O(\ln \varepsilon)$ и $\left|\zeta_{+}-\zeta_{*}\right|=O\left(\varepsilon^{3 / 4} \ln \varepsilon\right)$, имеем:

$$
\left|\tau_{+}-\tau_{*}\right|=O\left(\varepsilon^{3 / 4}|\ln \varepsilon|\right) .
$$

Поэтому выполнено (11.15). Условия (11.11), (11.12) легко проверяются. Лемма 11.13 доказана.

\section{§ 4. Прохождение через четкий существенный резонанс}

Пусть I - компонента связности $\mathscr{D}_{C R}$. В этом параграфе мы обсудим прохождение траектории через I. Согласно предложению 11.8, I соответствует некоторому "почти резонансу" (11.5). Будем предполагать, что существует $\eta^{0} \in \mathbf{I}$ такое, что $k \nu_{0}+k_{0}=0, \nu_{0}=\nu\left(\eta^{0}\right)$. Случай, когда $\left.\nu(\eta)\right|_{\mathbf{I}} \neq \nu_{0}$, более простой и может быть рассмотрен аналогично. Для краткости положим $\eta^{0}=0$.

ЛЕмма 11.14. Предположим, что выполнено условие $\mathbf{H}_{\mathbf{1}} \mathbf{1}$. Тогда траекторию можно продолжить через I. Это требует времени $t=O(|I||\ln \varepsilon| / \varepsilon)$.

ДокАзАтЕЛьство. Конструкция правых совместных квазитраекторий примерно та же, что и в $\S 3$. В отличие от ситуации с прохождением через нерезонансный интервал слагаемое $\left(\tau_{+}-\tau-t_{+}\right) \mathbf{H}_{\zeta}\left(\varepsilon \rho_{+}, \zeta\right)$ может мешать возрастанию переменной $\rho$, если $\mathbf{H}_{\zeta}\left(\varepsilon \rho_{+}, \zeta\right)$ положительно. Поэтому важно следить за изменением переменной $\zeta$.

Обозначим

$$
\nu_{0}=\nu(0)=-\frac{k_{0}}{k}, \quad \nu_{0}^{\prime}=\frac{d \nu}{d \eta}(0), \quad \nu_{0}^{\prime \prime}=\frac{d^{2} \nu}{d \eta^{2}}(0),
$$

где, ввиду $\mathbf{H}_{\mathbf{0}} \mathbf{4},\left|\nu_{0}^{\prime}\right|+\left|\nu_{0}^{\prime \prime}\right|>2 c_{\nu}$. Положим

$$
c_{\Theta}=\sup _{\eta \in \bar{D},(\xi, \tau) \in \mathbb{T}^{2}} \Theta_{\xi}(\eta, \xi, \tau) .
$$

Определим некоторую динамическую задачу с управлением и шумом. 
ЗАДАчА 1. Рассмотрим дискретную динамическую систему

$$
\begin{aligned}
\mu_{n+1}-\mu_{n} & =\sqrt{\varepsilon}\left(u_{n}-t_{n+1} \mathbf{H}_{\zeta}\left(\sqrt{\varepsilon} \mu_{n}, \zeta_{n}\right)\right), \\
\zeta_{n+1}-\zeta_{n} & =\nu\left(\sqrt{\varepsilon} \mu_{n}\right) t_{n+1}+\beta_{n} .
\end{aligned}
$$

Здесъ для любого $n$

$$
K_{0}<\lambda t_{n}+\ln \varepsilon<K_{0}+\bar{K}, \quad\left|\beta_{n}\right|<c^{-1} \varepsilon^{3 / 4}|\ln \varepsilon| .
$$

Переменная $u_{n}$ является управлением, которое в соответствии с нашими нуждами берется в одном из интервалов

$$
\left(-c_{\Theta}-b,-5 b\right) \quad \text { или }\left(5 b, c_{\Theta}+b\right) .
$$

Величины $u_{n}$ из этих интервалов и величины $t_{n}, \beta_{n}$, удовлетворяющие (11.21), произвольны (т.е. их точные значения неизвестны). Этот произвол при желании можно считать шумом. Цель состоит в том, чтобы построить траекторию, начинаюшуюся в точке $\left(\mu_{0}, \zeta_{0}\right)$ и заканчиваюшуюся в некоторой точке $\left(\mu_{N}, \zeta_{N}\right)$, где

$$
\widehat{\mathbf{I}}=\{\mu \in \mathbb{R}: \sqrt{\varepsilon} \mu \in \mathbf{I}\} \subset\left[\mu_{0}, \mu_{N}\right] .
$$

ПРЕДЛОЖЕНИЕ 11.15. Задача 1 имеет решение с $N=O(|\mathbf{I}| / \varepsilon)$.

ПРЕДЛОЖЕНИЕ 11.16. Предположим, что задача 1 имеет решение. Тогда лемма 11.14 справедлива с $t=O(N \ln \varepsilon)$.

Доказательство предложения 11.15. Получим некоторые вспомогательные оценки. Для некоторой большой постоянной $C_{1}$ рассмотрим множество

$$
\widehat{\mathbf{I}}^{c r}=\left\{\mu \in \widehat{\mathbf{I}}:\left|\nu(\sqrt{\varepsilon} \mu)-\nu_{0}\right| \leqslant C_{1} \varepsilon^{3 / 4}\right\} .
$$

ПРЕДЛОЖЕНИЕ 11.17. Предположим, что $\left|\nu_{0}^{\prime}\right|+\left|\nu_{0}^{\prime \prime}\right| \geqslant 2 c_{\nu}$. Тогда

(a) $|\widehat{\mathbf{I}}|=O\left(\varepsilon^{-3 / 8}\right)$,

(b) $\widehat{\mathbf{I}}^{\text {cr }}$ состоит из одного или двух интервалов $\widehat{\mathbf{I}}_{j}^{c r}, j=1,2$, и $\left|\widehat{\mathbf{I}}_{j}^{c r}\right|=$ $O\left(\varepsilon^{-1 / 8}\right)$.

\section{ДОКАЗАТЕЛЬСТВО ПРЕДЛОЖЕНИЯ 11.17.}

УтВеРЖДЕНИЕ. Пусть $u, v, c_{u}, c_{v}, s$ - постоянные такие, что $c_{u}, c_{v}, s$ положительнь, $|u| / c_{u}+|v| / c_{v} \geqslant 2$, и пусть $I \subset \mathbb{R}-$ интервал. Предположим, что

$$
\left|u x+v x^{2}\right|<s \quad \text { для любого } \quad x \in I .
$$

Тогда длина интервала I не превосходит $\max \left\{4 s / c_{u}, \sqrt{4 s / c_{v}}\right\}$.

Простое доказательство утверждения опустим.

Теперь, чтобы доказать предложение 11.17 , достаточно заметить, что

$$
\begin{gathered}
\widehat{\mathbf{I}} \subset\left\{\mu \in \mathbb{R}:\left|k\left(\nu_{0}^{\prime} \mu+\frac{1}{2} \nu_{0}^{\prime \prime} \sqrt{\varepsilon} \mu^{2}+O\left(\varepsilon \mu^{3}\right)\right)\right| \leqslant 2 \varepsilon^{-1 / 4}\right\}, \\
\widehat{\mathbf{I}}^{c r} \subset\left\{\mu \in \mathbb{R}:\left|\nu_{0}^{\prime} \mu+\frac{1}{2} \nu_{0}^{\prime \prime} \sqrt{\varepsilon} \mu^{2}+O\left(\varepsilon \mu^{3}\right)\right| \leqslant C_{1} \varepsilon^{1 / 4}\right\} .
\end{gathered}
$$

Предложение 11.17 доказано. 
Рассмотрим систему

$$
\left\{\begin{array}{l}
\dot{\mu}=v-\mathbf{H}_{\zeta}(\sqrt{\varepsilon} \mu, \zeta), \\
\dot{\zeta}=\frac{1}{\sqrt{\varepsilon}}\left(\nu(\sqrt{\varepsilon} \mu)-\nu_{0}\right)+\sqrt{\varepsilon} \mathbf{H}_{\eta}(\sqrt{\varepsilon} \mu, \zeta), \quad v=\frac{2 b \lambda}{|\ln \varepsilon|} .
\end{array}\right.
$$

Постоянная $v$ мала и положительна. Эта система гамильтонова с симплектической структурой $d \mu \wedge d \zeta$ и гамильтонианом

$$
\chi(\mu, \zeta)=\frac{1}{\varepsilon}\left(E(\sqrt{\varepsilon} \mu)-E(0)-\nu_{0} \sqrt{\varepsilon} \mu\right)+\mathbf{H}(\sqrt{\varepsilon} \mu, \zeta)-v \zeta .
$$

Эта система будет использоваться для сравнения ее траекторий с траекториями задачи 1.

Гамильтониан (11.24) многозначен на цилиндре

$$
Z=\{(\mu, \zeta): \mu \in \widehat{\mathbf{I}}, \zeta \bmod 1\} .
$$

Но соответствующее гамильтоново векторное поле 1-периодично по $\zeta$ и, следовательно, однозначно на $Z$. Чтобы сделать $\chi$ однозначным, будем считать, что $\chi: Z \rightarrow \mathbb{R} /(v \mathbb{Z})$. Другими словами, возьмем $\chi \bmod v$. Заметим также, что, ввиду предложения 11.1, 1-периодическая по $\zeta$ функция Н приблизительно $1 / k$-периодична по $\zeta$ :

$$
\mathbf{H}(\eta, \zeta+1 / k)-\mathbf{H}(\eta, \zeta)=O\left(\eta^{j} \ln \varepsilon\right) \quad \text { для } C^{j} \text {-гладкого } H_{1} .
$$

Пусть $\partial_{-} Z$ и $\partial_{+} Z-$ компоненты связности границы $\partial Z$ :

$$
\partial_{-} Z \cup \partial_{+} Z=\partial Z,\left.\quad \mu\right|_{\partial_{-} Z}<0<\left.\mu\right|_{\partial_{+} Z}
$$

Система (11.23) интегрируема. Любая траектория, начинающаяся на $\partial_{-} Z$, либо достигает $\partial_{+} Z$, либо асимптотически стремится к положению равновесия (11.23).

В случае, когда $\widehat{\mathbf{I}}^{c r}$ состоит из двух компонент связности, пусть $\mu_{0}-$ некоторое число, их разделяющее, и

$$
S_{0}=\left\{(\mu, \zeta) \in Z: \mu=\mu_{0}\right\}
$$

ПРедЛОЖЕНИЕ 11.18. Предположим, что $\widehat{\mathbf{I}}^{c r}$ связно (соответственно несвязно). Тогда система (11.23) имеет траекторию

$$
\gamma(t)=(\mu(t), \zeta(t)), \quad t \in\left[0, t_{*}\right],\left.\quad \chi\right|_{\gamma}=\chi_{*}
$$

(соответственно траектории $\left.\gamma^{ \pm}(t)=\left(\mu^{ \pm}(t), \zeta^{ \pm}(t)\right), t \in\left[0, t_{*}^{ \pm}\right],\left.\chi\right|_{\gamma^{ \pm}}=\chi_{*}^{ \pm}\right)$ такую, что

1) $\mu(0) \in \partial_{-} Z, \mu\left(t_{*}\right) \in \partial_{+} Z$ (соответственно $\mu^{-}(0) \in \partial_{-} Z, \mu^{-}\left(t_{*}^{-}\right) \in S_{0}$ $\left.\mu^{+}(0) \in S_{0}, \mu^{+}\left(t_{*}^{+}\right) \in \partial_{+} Z\right)$,

2) $t_{*}<|\widehat{\mathbf{I}}| /(c v)$ (соответственно $\left.t_{*}^{-}, t_{*}^{+}<|\widehat{\mathbf{I}}| /(c v)\right)$ для некоторой постоянной $c$,

3) утверждения 1) и 2) выполнены для любой траектории с энергией из интервала $\left[\chi_{*}-c_{1} v^{2}, \chi_{*}+c_{1} v^{2}\right]$ (соответственно из интервалов $\left[\chi_{*}^{ \pm}-c_{1} v^{2}, \chi_{*}^{ \pm}+\right.$ $\left.\left.c_{1} v^{2}\right]\right)$. 
ЗАмечАниЕ 11.19. Ввиду (11.25) величину $\chi_{*}$ в 3$)$ можно брать $\bmod v / k$.

ДокАЗАТЕЛЬСтво. Мы рассмотрим только случай связного $\widehat{\mathbf{I}}^{c r}$. Для несвязного $\widehat{\mathbf{I}}^{\text {cr }}$ доказательство то же. Пусть

$$
M=\max _{\zeta \in \mathbb{T}} \mathbf{H}(0, \zeta), \quad A=\max _{\zeta \in \mathbb{T}} \mathbf{H}_{\zeta \zeta}(0, \zeta) .
$$

УТВЕРЖДЕНИЕ. Для любого $h \in\left(M-v^{2} /(8 A), M\right)$ любое $\widehat{\zeta}$ maкое, что $\mathbf{H}(0, \widehat{\zeta})=h$, удовлетворяет неравенству $\left|\mathbf{H}_{\zeta}(0, \widehat{\zeta})\right| \leqslant v / 2$.

Доказательство утверждения опустим (см. [66]).

Пусть $\gamma(t)$ - траектория с начальными условиями

$$
(\mu, \zeta)=\left(0, \zeta^{0}\right), \quad \mathbf{H}\left(0, \zeta^{0}\right)=h \in\left(M-\frac{3 v^{2}}{32 A}, M-\frac{v^{2}}{32 A}\right) .
$$

Согласно приведенному выше утверждению, пока

$$
\gamma(t) \in \widehat{\mathbf{I}}^{c r} \cap\left\{(\mu, \zeta): \mathbf{H}(0, \zeta) \in\left(M-\frac{v^{2}}{8 A}, M\right)\right\},
$$

выполняются соотношения: $\dot{\mu}>v / 2, \dot{\zeta}=O\left(\varepsilon^{1 / 4}\right)$. Напомним, что ввиду предложения $11.17(\mathrm{~b})\left|\widehat{\mathbf{I}}^{c r}\right|=O\left(\varepsilon^{-1 / 8}\right)$. Таким образом, $\gamma(t)$ проходит $\widehat{\mathbf{I}}^{c r}$ через прямоугольник (11.26) со скоростью в направлении $\mu$ большей, чем $v / 2$.

Вне $\widehat{\mathbf{I}}^{c r} \dot{\zeta}$ отделено от нуля. Следовательно, в среднем, $\mu$ возрастает со скоростью $v$. Предложение 11.18 доказано.

ПРЕДЛОЖЕНИЕ 11.20. Для $C^{6}$-гладкого $H_{1} u \mu \in \mathbf{I}$

$$
\begin{aligned}
& \chi\left(\mu_{n+1}, \zeta_{n+1}\right)-\chi\left(\mu_{n}, \zeta_{n}\right)+v \nu_{0} t_{n+1} \\
& \quad=\left(\nu\left(\sqrt{\varepsilon} \mu_{n}\right)-\nu_{0}\right)\left(u_{n}-v t_{n+1}\right)+|\ln \varepsilon| O\left(\varepsilon^{3 / 4}+\left(\nu(\sqrt{\varepsilon} \mu)-\nu_{0}\right)^{2}\right) .
\end{aligned}
$$

ДоКАЗАТЕЛЬСТВо ПРЕДЛОЖЕНИЯ 11.20. Ввиду (11.24) имеем

$$
\begin{aligned}
\chi\left(\mu_{n+1}, \zeta_{n+1}\right)-\chi\left(\mu_{n}, \zeta_{n}\right)+v \nu_{0} t_{n+1}=\mathbf{A}+\mathbf{B}+\mathbf{C}+\mathbf{D}, \\
\mathbf{A}=\frac{1}{\varepsilon}\left(E\left(\sqrt{\varepsilon} \mu_{n+1}\right)-E\left(\sqrt{\varepsilon} \mu_{n}\right)\right), \\
\mathbf{B}=-\frac{1}{\sqrt{\varepsilon}} \nu_{0}\left(\mu_{n+1}-\mu_{n}\right), \\
\mathbf{C}=\mathbf{H}\left(\sqrt{\varepsilon} \mu_{n+1}, \zeta_{n+1}\right)-\mathbf{H}\left(\sqrt{\varepsilon} \mu_{n}, \zeta_{n}\right), \\
\mathbf{D}=-v\left(\zeta_{n+1}-\nu_{0} t_{n+1}-\zeta_{n}\right) .
\end{aligned}
$$

Используя (11.19), (11.20), получаем:

$$
\begin{aligned}
& \mathbf{A}=\nu\left(\sqrt{\varepsilon} \mu_{n}\right)\left(u_{n}-t_{n+1} \mathbf{H}_{\zeta}\left(\sqrt{\varepsilon} \mu_{n}, \zeta_{n}\right)\right)+O\left(\varepsilon \ln ^{2} \varepsilon\right), \\
& \mathbf{B}=-\nu_{0}\left(u_{n}-t_{n+1} \mathbf{H}_{\zeta}\left(\sqrt{\varepsilon} \mu_{n}, \zeta_{n}\right)\right), \\
& \mathbf{D}=-v\left(\nu\left(\sqrt{\varepsilon} \mu_{n}\right)-\nu_{0}\right) t_{n+1}+O\left(\varepsilon^{3 / 4}\right) .
\end{aligned}
$$


Чтобы оперировать с $\mathbf{C}$, используем оценки

$$
\mu_{n+1}-\mu_{n}=O(\sqrt{\varepsilon} \ln \varepsilon), \quad \mathbf{H}_{\zeta}\left(\sqrt{\varepsilon} \mu, \zeta+\nu_{0} t_{n+1}\right)-\mathbf{H}_{\zeta}(\sqrt{\varepsilon} \mu, \zeta)=O\left(\varepsilon^{3 / 2}\right) .
$$

Последняя оценка следует из предложения 11.1 для $C^{6}$-гладкого $H_{1}$, так как при $\sqrt{\varepsilon} \mu \in \mathbf{I}$ имеем $\delta=\left|\nu(\sqrt{\varepsilon} \mu)-\nu_{0}\right|<\varepsilon^{1 / 4}$. Получаем:

$$
\left.\mathbf{C}=\left(\nu\left(\sqrt{\varepsilon} \mu_{n}\right)-\nu_{0}\right) t_{n+1} \mathbf{H}_{\zeta}\left(\sqrt{\varepsilon} \mu_{n}, \zeta_{n}\right)+|\ln \varepsilon|\left(O\left(\varepsilon^{3 / 4}\right)+O\left(\nu(\sqrt{\varepsilon} \mu)-\nu_{0}\right)^{2}\right)\right) .
$$

Из этих оценок для $\mathbf{A}, \mathbf{B}, \mathbf{C}, \mathbf{D}$ вытекает (11.27). Предложение 11.20 доказано.

Так как $\lambda t_{n+1}=|\ln \varepsilon|+O(1)$, имеем:

$$
\begin{array}{lll}
u_{n}-v t_{n+1}>2 b & \text { для } & u_{n} \in\left[5 b, c_{\Theta}+b\right], \\
u_{n}-v t_{n+1}<-6 b & \text { для } & u_{n} \in\left[-c_{\Theta}-b,-5 b\right] .
\end{array}
$$

Таким образом, используя $u_{n}$ можно обеспечить рост или убывание $\chi \bmod v / k$ на траектории. В частности, можно использовать управление $u_{0}, u_{1}, \ldots$, чтобы сначала сделать $\chi$ близким к $\chi_{*}$ :

$$
\chi\left(\mu_{n}, \zeta_{n}\right)=\left(\chi_{*}+O(\sqrt{\varepsilon})\right) \bmod v / k, \quad n=n_{0} \leqslant v \varepsilon^{-1 / 2},
$$

а затем сохранять это условие для $n>n_{0}$ при условии

$$
\left|\nu\left(\sqrt{\varepsilon} \mu_{n}\right)-\nu_{0}\right|>C \varepsilon^{3 / 4}|\ln \varepsilon|, \quad \text { т.e. } \quad \mu \notin \widehat{\mathbf{I}}^{c r} .
$$

Так как последовательность $\chi\left(\mu_{k}, \zeta_{k}\right), k>n_{0}$, лишь слегка осциллирует, траектория $\left(\mu_{n}, \zeta_{n}\right)$ следует кривой $\gamma$ из предложения 11.18 при условии (11.28). Чтобы войти в полосу

$$
S=\left\{(\mu, \zeta): \mu \in \mathbf{I}^{c r}\right\}
$$

необходимо

$$
N_{1} \leqslant O\left(\left|\widehat{\mathbf{I}}^{c r}\right| / \sqrt{\varepsilon}\right)
$$

шагов (итераций сепаратрисного отображения). Действительно, (11.29) следует из того факта, что средний прирост $\mu$ на этой части траектории имеет порядок $O(\sqrt{\varepsilon})$.

Когда траектория $\left(\mu_{n}, \zeta_{n}\right)$ входит в полосу $S$, слагаемые в $(11.27)$, отвечающие за ошибки, могут доминировать. В этом случае мы выбираем управление $u \in\left[5 b, c_{\Theta}+b\right]$. На $\gamma \cap S$ из уравнения (11.23) следует, что

$$
\dot{\mu}=v-\mathbf{H}_{\zeta}\left(\eta^{0}, \zeta\right)>v / 2
$$

Более того, согласно предложению 11.18 это неравенство остается верным, пока

$$
(\mu, \zeta) \in\left\{\left|\chi(\mu, \zeta)-\chi_{*}\right|<c_{1} v^{2}\right\} \cap S, \quad c_{1}=1 /(32 A) .
$$

Обратимся к траектории $\left(\mu_{n}, \zeta_{n}\right)$. Используя (11.19) и $(11.30)$, видим, что из (11.31) следует, что

$$
\mu_{n+1}-\mu_{n}>\sqrt{\varepsilon}\left(5 b-\frac{2|\ln \varepsilon|}{\lambda} \mathbf{H}_{\zeta}\left(\eta^{0}, \zeta_{n}\right)\right)>\frac{3}{2} \sqrt{\varepsilon} b .
$$


Таким образом, $\mu_{k}$ возрастает, пока $(\mu, \zeta)=\left(\mu_{k}, \zeta_{k}\right)$ удовлетворяет (11.31). Чтобы пройти через $S$, требуется не более чем

$$
N_{2}=\frac{C \varepsilon^{-1 / 8}}{\sqrt{\varepsilon} b}=\frac{C}{\varepsilon^{5 / 8} b}
$$

шагов. В течение этого времени $\chi$ изменится не более чем на $N_{2} O\left(\varepsilon^{3 / 4}|\ln \varepsilon|\right)=$ $O\left(\varepsilon^{1 / 8} \ln \varepsilon\right)$. Это значит, что если траектория входит в $S$ с $\chi=\left(\chi_{*}+O(\sqrt{\varepsilon})\right) \bmod$ $v / k$, то она останется в области $(11.31)$, выходя из $S$.

Наконец, для полного количества $N$ шагов на траектории имеем оценку:

$$
N=2 N_{1}+N_{2}=O(|\widehat{\mathbf{I}}| / \sqrt{\varepsilon})=O(|\mathbf{I}| / \varepsilon) .
$$

Предложение 11.20 доказано.

ДОКАЗАТЕЛЬСТВО ПРЕДЛОЖЕНИЯ 11.16. ИспольЗуя лемму 11.13, можно построить траекторию такую, что на соответствующей последовательности правых совместных квазитраекторий

$$
\left(\bar{\Omega}_{j}, \bar{\Omega}_{j+1}\right),\left(\bar{\Omega}_{j+1}, \bar{\Omega}_{j+2}\right), \ldots
$$

в соответствии с нашими нуждами $\widehat{\Theta}_{\zeta}\left(\varepsilon \bar{\rho}_{n}, \bar{\zeta}_{n}, \bar{\tau}_{n}\right), n=j, j+1, \ldots$, лежит или в $\left[6 b, c_{\Theta}\right]$, или в $\left[-c_{\Theta},-6 b\right]$. При $\left|\varepsilon \bar{\rho}_{n}-\eta^{0}\right|<c \varepsilon^{1 / 4}$ (напомним, что $\eta^{0}=0$ ), комбинируя (10.13) с оценками

$$
\left|\bar{\rho}_{n}-\rho_{n}\right|<2 b / b_{\rho}, \quad\left|\bar{\zeta}_{n}-\zeta_{n}\right|<2 b / b_{\zeta}, \quad\left|\bar{\tau}_{n}-\tau_{n}\right|<2 b / b_{\tau},
$$

получаем:

$$
\begin{aligned}
& \bar{\rho}_{n+1}=\bar{\rho}_{n}-\widehat{\Theta}_{\zeta}\left(\varepsilon \bar{\rho}_{n}, \bar{\zeta}_{n}, \bar{\tau}_{n}\right)+\left(\bar{\tau}_{n+1}-\bar{\tau}_{n}-\bar{t}_{n+1}\right) \mathbf{H}_{\zeta}\left(\varepsilon \bar{\rho}_{n}, \bar{\zeta}_{n}\right)+\widetilde{\alpha}_{n}, \\
& \bar{\zeta}_{n+1}=\bar{\zeta}_{n}+\nu\left(\varepsilon \bar{\rho}_{n}\right) \bar{t}_{n+1}+\varepsilon\left(\bar{\tau}_{n+1}-\bar{\tau}_{n}-\bar{t}_{n+1}\right) \mathbf{H}_{\eta}\left(\varepsilon \bar{\rho}_{n}, \bar{\zeta}_{n}\right)+\widetilde{\beta}_{n}
\end{aligned}
$$

где $\left|\widetilde{\alpha}_{n}\right|<b / 2,\left|\widetilde{\beta}_{n}\right|<c^{-1} \varepsilon^{3 / 4}|\ln \varepsilon| / 2$.

Пусть $\mu_{n}$ удовлетворяет уравнениям

$$
\varepsilon \bar{\rho}_{n}=\sqrt{\varepsilon} \mu_{n}+\varepsilon \bar{\tau}_{n} \mathbf{H}_{\zeta}\left(\sqrt{\varepsilon} \mu_{n}, \bar{\zeta}_{n}\right), \quad n=j, j+1, \ldots
$$

Тогда

$$
\begin{aligned}
\mu_{n+1} & =\mu_{n}-\widehat{\Theta}_{\zeta}\left(\varepsilon \bar{\rho}_{n}, \bar{\zeta}_{n}, \bar{\tau}_{n}\right)-\bar{t}_{n+1} \mathbf{H}_{\zeta}\left(\sqrt{\varepsilon} \mu_{n}, \bar{\zeta}_{n}, \bar{\tau}_{n}\right)+\alpha_{n}, \\
\alpha_{n} & =\widetilde{\alpha}_{n}-\left(\bar{\tau}_{n}+\bar{t}_{n+1}\right) \delta_{1}+\bar{\tau}_{n+1} \delta_{2}, \\
\delta_{1} & =\mathbf{H}_{\zeta}\left(\sqrt{\varepsilon} \mu_{n}+\varepsilon \bar{\tau}_{n} \mathbf{H}_{\zeta}\left(\sqrt{\varepsilon} \mu_{n}, \bar{\zeta}_{n}\right), \bar{\zeta}_{n}\right)-\mathbf{H}_{\zeta}\left(\sqrt{\varepsilon} \mu_{n}, \bar{\zeta}_{n}\right), \\
\delta_{2} & =\mathbf{H}_{\zeta}\left(\sqrt{\varepsilon} \mu_{n}+\varepsilon \bar{\tau}_{n} \mathbf{H}_{\zeta}\left(\sqrt{\varepsilon} \mu_{n}, \bar{\zeta}_{n}\right), \bar{\zeta}_{n}\right)-\mathbf{H}_{\zeta}\left(\sqrt{\varepsilon} \mu_{n+1}, \bar{\zeta}_{n+1}\right) .
\end{aligned}
$$

Имеем: $\delta_{1}=O\left(\varepsilon^{3 / 4}\right), \delta_{2}=O\left(\varepsilon^{1 / 4} \ln \varepsilon\right)+O(\sqrt{\varepsilon} \mu \ln \varepsilon)$. Действительно, первая оценка очевидна. Вторая следует из равенств

$$
\begin{gathered}
\mu_{n+1}-\mu_{n}=O(\ln \varepsilon), \quad \bar{\zeta}_{n+1}-\bar{\zeta}_{n}=-k_{0} / k+O(\sqrt{\varepsilon} \mu \ln \varepsilon), \\
\mathbf{H}_{\zeta}\left(\sqrt{\varepsilon} \mu, \zeta+k_{0} / k\right)-\mathbf{H}_{\zeta}(\sqrt{\varepsilon} \mu, \zeta)=O(\varepsilon) .
\end{gathered}
$$


Последнее равенство следует из предложения 11.1 для $C^{j}$-гладкого $H_{1}, j>5$.

Таким образом, $\left|\alpha_{n}\right|<b$ и величина $u_{n}=-\widehat{\Theta}_{\zeta}\left(\varepsilon \bar{\rho}_{n}, \bar{\zeta}_{n}, \bar{\tau}_{n}\right)+\alpha_{n}$ лежит в одном из интервалов (11.22), откуда следует (11.19). Так как

$$
\nu\left(\varepsilon \bar{\rho}_{n}\right)=\nu(0)+\nu_{0}^{\prime} \sqrt{\varepsilon} \mu_{n}+\frac{\nu_{0}^{\prime \prime}}{2} \varepsilon \mu_{n}^{2}+O(\varepsilon)+\varepsilon^{3 / 2} O\left(\mu_{n}^{3}\right)
$$

получаем (11.19)-(11.20). Предложение 11.16 доказано.

\section{§ 5. Прохождение через нечеткий интервал}

Зафиксируем резонанс $\eta=\eta^{0}$, порождающий нечеткий интервал $\mathbf{I} \subset \mathscr{D}_{V}$. Далее без ограничения общности будем считать, что $\eta^{0}=0$. Обозначим $\nu_{0}=$ $\nu(0)=p_{0} / q_{0}$, где $p_{0} \in \mathbb{Z}, q_{0} \in \mathbb{N}, q_{0}<2 / c_{*}$, и дробь $p_{0} / q_{0}$ несократима.

Лемма 11.21. Предположим, что условие $\mathbf{H}_{\mathbf{1}} \mathbf{2}$ выполнено для нечеткого резонанса $\eta=0$. Тогда допустимую траекторию можно продолжить через соответствующий нечеткий интервал $\mathbf{I} \subset \mathscr{D}_{V}$. Это требует времени $t=$ $O(|\ln \varepsilon| / \varepsilon)$.

Ввиду $\mathbf{H}_{\mathbf{1}} \mathbf{2}$, функции $F_{ \pm}$удовлетворяют условиям 1$\left.)-4\right)$ предложения 11.10. Для достаточно малых $c^{\prime}, c^{\prime \prime}$ функции

$$
\begin{aligned}
& F_{c^{\prime}, c^{\prime \prime},+}(\zeta)=\max _{u \in\left[-q_{0}, 0\right]}\left\{\Theta_{\xi}\left(0, \zeta-\nu_{0} u, u\right):\left(0, \zeta-\nu_{0} u, u\right) \in J_{2 c^{\prime}, 2 c^{\prime \prime}}\right\}, \\
& F_{c^{\prime}, c^{\prime \prime},-}(\zeta)=\min _{u \in\left[-q_{0}, 0\right]}\left\{\Theta_{\xi}\left(0, \zeta-\nu_{0} u, u\right):\left(0, \zeta-\nu_{0} u, u\right) \in J_{2 c^{\prime}, 2 c^{\prime \prime}}\right\}
\end{aligned}
$$

также удовлетворяют этим условиям.

Пусть $\gamma_{0}^{ \pm}=\gamma_{0}^{ \pm}(\zeta)$ таковы, что $F_{c^{\prime}, c^{\prime \prime}, \pm}(\zeta)=\Theta_{\xi}\left(0, \zeta-\nu_{0} \gamma_{0}^{ \pm}, \gamma_{0}^{ \pm}\right)$. Так как $\left(0, \zeta-\nu_{0} \gamma_{0}^{ \pm}, \gamma_{0}^{ \pm}\right) \in J_{2 c^{\prime}, 2 c^{\prime \prime}}$, имеем: $\gamma_{0}^{ \pm}(\zeta)=\Psi^{0, \zeta, \gamma_{0}^{ \pm}(\zeta)}(0, \zeta, 0)$. Функции $\gamma_{0}^{ \pm}(\zeta)$ кусочно $C^{j-1}$-гладкие.

Для малого $|\eta|$ положим

$$
\begin{gathered}
\widehat{F}_{c^{\prime}, c^{\prime \prime}, \pm}(\eta, \zeta)=\Theta_{\xi}\left(\eta, \zeta-\nu(\eta) \gamma^{ \pm}, \gamma^{ \pm}\right), \\
\gamma^{ \pm}=\gamma^{ \pm}(\eta, \zeta)=\Psi^{0, \zeta, \gamma_{0}^{ \pm}(\zeta)}(\eta, \zeta, 0),
\end{gathered}
$$

так что $\widehat{\Theta}_{\tau}\left(\eta, \zeta, \gamma^{ \pm}\right)=0$ и $\left(\eta, \zeta-\nu(\eta) \gamma^{ \pm}, \gamma^{ \pm}\right) \in J_{c^{\prime}, c^{\prime \prime}}$. Функции $\widehat{F}_{c^{\prime}, c^{\prime \prime}, \pm}$ гладкие по $\eta$ и при всех малых $|\eta|$ удовлетворяют условиям 1)-4) предложения 11.10 с $c_{F} / 2$ вместо $c_{F}$.

Будем считать, что $c^{\prime}, c^{\prime \prime},|\eta|$, с которыми мы имеем дело, достаточно малы в этом смысле. Для краткости не будем писать индексы $c^{\prime}, c^{\prime \prime}$. В частности, вместо $\widehat{F}_{c^{\prime}, c^{\prime \prime}, \pm}$ пишем $\widehat{F}_{ \pm}$.

Предположим, что допустимая траектория $\mathbb{O}=\left(\Omega_{-k}, \ldots, \Omega_{k}\right)$ с кодом $\overline{\mathbb{O}}=$ $\left(\bar{\Omega}_{-k}, \ldots, \bar{\Omega}_{k}\right)$ вошла в нечеткий интервал, т.е. $\varepsilon \bar{\rho}_{k} \in \mathbf{I}, \bar{\rho}_{k}<0$. Отметим, что, ввиду предложения $11.5, \mathbf{I} \subset\left[-C_{\nu} /\left(q_{0} K\right), C_{\nu} /\left(q_{0} K\right)\right]$, где $C_{\nu}-$ постоянная Липшица для $\nu^{-1}(\eta)$ около точки $\eta=0$ и $K$ удовлетворяет (11.4).

Далее $F_{ \pm}$играют роль функций $\Theta_{\zeta}$ в уравнениях динамики. 
ЛЕмма 11.22. Предположим, что условие $\mathbf{H}_{\mathbf{1}} \mathbf{2}$ выполнено для нечеткого $\eta=0$. Тогда для любого $\left|\varepsilon \rho_{k}\right|<C_{\nu} /\left(q_{0} K\right) u * \in\{+,-\}$ существует правая совместная квазитраектория $\left(\bar{\Omega}_{k}, \bar{\Omega}_{k+1}\right)$ и целое $\mathbf{t}$

$$
\mathbf{t}-\left[\frac{1}{\lambda}\left(K_{0}-\ln \varepsilon\right)\right] \in\left\{1,2, \ldots, 2 q_{0}\right\},
$$

где $[\cdot]: \mathbb{R} \rightarrow \mathbb{Z}-$ челая часть аргумента, такие, что

$$
\begin{gathered}
\widehat{\Theta}_{\zeta}\left(\varepsilon \bar{\rho}_{k+1}, \bar{\zeta}_{k+1}, \bar{\tau}_{k+1}\right)=\widehat{F}_{*}\left(\varepsilon \rho_{k}, \zeta_{k}+\nu\left(\varepsilon \rho_{k}\right) \mathbf{t}\right)+\alpha_{*}, \\
\left|\alpha_{*}\right|<d, \quad d=O\left(\varepsilon^{3 / 4}|\ln \varepsilon|\right) .
\end{gathered}
$$

Мы докажем лемму 11.22 в $\S 6$, а теперь определим еще одну динамическую задачу.

ЗАДАчА 2. Рассмотрим дискретную динамическую систему

$$
\begin{aligned}
\mu_{n+1}-\mu_{n}= & -\sqrt{\varepsilon}\left(v_{n}+t_{n+1} \mathbf{H}_{\zeta}\left(\sqrt{\varepsilon} \mu_{n}, \zeta_{n}\right)+\alpha_{n}\right), \\
\zeta_{n+1}-\zeta_{n} & =\nu\left(\sqrt{\varepsilon} \mu_{n}\right) t_{n+1}+\beta_{n}
\end{aligned}
$$

Здесъ для любого $n$

$$
\begin{gathered}
\mathbf{t}_{n} \leqslant t_{n} \leqslant \mathbf{t}_{n}+2 q_{0}, \quad t_{n} \in \mathbb{Z}, \\
\mathbf{t}_{n}-\left[\frac{1}{\lambda}\left(K_{0}-\ln \varepsilon\right)\right] \in\left\{1,2, \ldots, 2 q_{0}\right\}, \\
\left|\beta_{n}\right|<C \varepsilon^{3 / 4}|\ln \varepsilon|, \quad\left|\alpha_{n}\right|<d .
\end{gathered}
$$

Постоянная $d$ мала ${ }^{13}$. Переменнье $\mathbf{t}_{n}, v_{n}$ следует считать управлением, где в соответствии с нашими нуждами $\mathbf{t}_{n}$, удовлетворяющее (11.37), может выбиратъся произвольно, а $v_{n}$ может выбираться равным одной из следующих двух величин:

$$
\widehat{F}_{ \pm}\left(\sqrt{\varepsilon} \mu_{n-1}, \zeta_{n-1}+\nu\left(\sqrt{\varepsilon} \mu_{n-1}\right) \mathbf{t}_{n}\right) .
$$

Величины $\alpha_{n}, \beta_{n}$ маль и считаются шумом. Они и $t_{n}$ принимают произвольные значения в соответствующих интервалах. Цель состоит в том, чтобы построить траекторию, начинающуюся в произвольной точке $\left(\mu_{0}, \zeta_{0}\right)$, $\sqrt{\varepsilon} \mu_{0}>-1 /\left(q_{0} \bar{K}\right)$ и заканчиваюшуюся в точке $\left(\mu_{N}, \zeta_{N}\right), \sqrt{\varepsilon} \mu_{N}>1 /\left(q_{0} \bar{K}\right) c$ как можно меньшим $N$.

ЛЕмма 11.23. Задача 2 имеет решение с $N \leqslant 1 /(c \varepsilon \bar{K})$.

Лемма 11.24. Предположим, что задача 2 имеет решение. Тогда лемма 11.21 справедлива с $t=O(N \ln \varepsilon)$.

\footnotetext{
${ }^{13}$ Например, ниже нам потребуется, чтобы выполнялось неравенство $d<\left|F_{-}\right| / 4$, cM. (11.70).
} 
Рассуждения аналогичны тем, что были использованы в § 3. Докажем лемму для $*=+$.

Требуется удовлетворить (11.11)-(11.14) и условие (11.33). Снова для краткости положим $\Omega_{+}=\bar{\Omega}_{k+1}$.

1) Сначала рассмотрим вместо (11.33) уравнение

$$
\Theta_{\xi}\left(\varepsilon \rho_{k}, \zeta_{k}+\nu\left(\varepsilon \rho_{k}\right) t,-t\right)=\widehat{F}_{+}\left(\varepsilon \rho_{k}, \zeta_{k}+\nu\left(\varepsilon \rho_{k}\right) \mathbf{t}\right)
$$

с неизвестным целым $t \in\left[\mathbf{t}, \mathbf{t}+2 q_{0}\right]$.

Согласно определению $\widehat{F}_{+}$, существует $t=\widehat{t} \in\left[\mathbf{t}, \mathbf{t}+2 q_{0}\right]$ такое, что

$$
\left(\varepsilon \rho_{k}, \zeta_{k}+\nu\left(\varepsilon \rho_{k}\right) \widehat{t},-\widehat{t}\right) \in J_{2 c^{\prime}, 2 c^{\prime \prime}} \quad \text { и равенство (11.40) справедливо. }
$$

2) Определим $t_{+}, \tau_{*}, \zeta_{*}$ с помощью (11.17). Тогда $t_{+}$удовлетворяет (11.14). Из условий (11.41) следует, что

$$
\left(\varepsilon \rho_{k}, \zeta_{*}-\nu\left(\varepsilon \rho_{k}\right) \tau_{*}, \tau_{*}\right) \in J_{c^{\prime}, c^{\prime \prime}}, \quad \widehat{\Theta}_{\zeta}\left(\varepsilon \rho_{k}, \zeta_{*}, \tau_{*}\right)=\widehat{F}_{+}\left(\varepsilon \rho_{k}, \zeta_{k}+\nu\left(\varepsilon \rho_{k}\right) \mathbf{t}\right)
$$

3) Определим $\rho_{+}, \zeta_{+}$с помощью (11.18).

4) Найдем $\tau_{+}$из уравнения $\bar{\partial} \Theta\left(\varepsilon \rho_{+}, \zeta_{+}-\nu\left(\varepsilon \rho_{+}\right) \tau_{+}, \tau_{+}\right)=0$, которое эквивалентно равенству $\widehat{\Theta}_{\tau}\left(\varepsilon \rho_{+}, \zeta_{+}, \tau_{+}\right)=0$. Таким образом,

$$
\tau_{+}=\Psi^{\rho_{k}, \zeta_{*}, \tau_{*}}\left(\varepsilon \rho_{+}, \zeta_{+}, 0\right) .
$$

Так как $\tau_{*}=\Psi^{\rho_{k}, \zeta_{*}, \tau_{*}}\left(\varepsilon \rho_{k}, \zeta_{*}, 0\right)$ и $\left|\zeta_{+}-\zeta_{*}\right|=O\left(\varepsilon^{3 / 4} \ln \varepsilon\right)$, получаем:

$$
\left|\tau_{+}-\tau_{*}\right|=O\left(\varepsilon^{3 / 4}|\ln \varepsilon|\right) .
$$

Следовательно,$\left|\tau_{+}\right|<1$. Имеем:

$$
\left|\widehat{\Theta}_{\zeta}\left(\varepsilon \rho_{+}, \zeta_{+}, \tau_{+}\right)-\widehat{\Theta}_{\zeta}\left(\varepsilon \rho_{k}, \zeta_{*}, \tau_{*}\right)\right|=O\left(\varepsilon^{3 / 4} \ln \varepsilon\right)
$$

Теперь (11.11), (11.12) легко проверяются.

\section{§ 7. Доказательство леммы 11.23}

Предположим, что траектория (O) задачи 2 приходит в некоторую точку $(\mu, \zeta)=\left(\mu_{s}, \zeta_{s}\right)$, где $-1 /\left(q_{0} \bar{K}\right) \leqslant \sqrt{\varepsilon} \mu_{s} \leqslant 1 /\left(q_{0} \bar{K}\right)$.

\section{1. Шаг 1 (вдали от резонанса).}

Сначала рассмотрим случай

$$
\varepsilon^{1 / 6}<|\sqrt{\varepsilon} \mu|<1 /\left(q_{0} \bar{K}\right) .
$$

ПреДЛОЖенИЕ 11.25. Пустъ $H_{1}$ лежит в пространстве $C^{j}$. Тогда при достаточно мальх є из (11.42) вытекает, что $\left|t_{n+1} \mathbf{H}_{\zeta}\left(\sqrt{\varepsilon} \mu_{n}, \zeta_{n}\right)\right|<C|\ln \varepsilon|^{1-j}$. 
ДокАЗАТЕльство. Пусть $c_{\nu}$ - липшицева постоянная для $\nu(y)$ и $c_{\nu}^{\prime}=\frac{d \nu}{d y}(0)$. Ввиду $\mathbf{H}_{\mathbf{0}} \mathbf{4}, c_{\nu}^{\prime}>0$. Из неравенства (11.42) следует, что

$$
q_{0} \varepsilon^{1 / 6} c_{\nu}^{\prime} / 2<\left|q_{0} \nu(\sqrt{\varepsilon} \mu)-p_{0}\right|<c_{\nu} / \bar{K} .
$$

Для любого резонанса $\nu(\sqrt{\varepsilon} \mu)=p / q, p \in \mathbb{Z}, q \in \mathbb{N}$, удовлетворяющего (11.43), имеем:

$$
1 \leqslant\left|q_{0} p-p_{0} q\right|<c_{\nu} q / \bar{K} .
$$

Так как $\bar{K}$ имеет порядок $|\ln \varepsilon|$, то $q$ велико: $q>\bar{K} / c_{\nu}$.

Оценим $\mathbf{H}_{\zeta}(\sqrt{\varepsilon} \mu, \zeta)$ при $\mu$, удовлетворяющем (11.42). Согласно $(6.1),(6.2)$ имеем:

$$
\left|\mathbf{H}_{\zeta}(\sqrt{\varepsilon} \mu, \zeta)\right| \leqslant C_{\mathbf{H}} q^{-j} \leqslant C_{\mathbf{H}} c_{\nu}^{j} \bar{K}^{-j}
$$

для некоторой постоянной $C_{\mathbf{H}}$. Итак,

$$
\left|t_{n+1} \mathbf{H}_{\zeta}\left(\sqrt{\varepsilon} \mu_{n}, \zeta_{n}\right)\right|<\frac{2|\ln \varepsilon| c_{\nu}^{j} C_{\mathbf{H}}}{\lambda \bar{K}^{j}}<\frac{C}{|\ln \varepsilon|^{j-1}} .
$$

Возьмем $v_{n}=\widehat{F}_{-}\left(\sqrt{\varepsilon} \mu_{n-1}, \zeta_{n-1}+\nu\left(\sqrt{\varepsilon} \mu_{n-1}\right) \mathbf{t}_{n}\right)$. Тогда (11.34)-(11.35) можно заменить системой

$$
\begin{aligned}
\mu_{n+1}-\mu_{n} & =-\sqrt{\varepsilon}\left(\widehat{F}_{-}\left(\sqrt{\varepsilon} \mu_{n-1}, \zeta_{n-1}+\nu\left(\sqrt{\varepsilon} \mu_{n-1}\right) \mathbf{t}_{n}\right)+\widehat{\alpha}_{n}\right), \\
\zeta_{n+1}-\zeta_{n} & =\nu\left(\sqrt{\varepsilon} \mu_{n}\right) t_{n+1}+\beta_{n}
\end{aligned}
$$

с $\left|\beta_{n}\right|<C \varepsilon^{3 / 4}|\ln \varepsilon|$ и $\left|\widehat{\alpha}_{n}\right|<2 d$. Далее опустим крышки над $\alpha_{n}$.

Идея состоит в следующем. Стартуя с точки $\left(\mu_{s}, \zeta_{s}\right)$, для $k \in \mathbb{N}$ получим:

$$
\begin{gathered}
\zeta_{s+k-1}+\nu\left(\sqrt{\varepsilon} \mu_{s+k-1}\right) \mathbf{t}_{s+k} \approx \widehat{\zeta}+k \Delta, \\
\text { где } \Delta=\nu\left(\sqrt{\varepsilon} \mu_{s}\right)\left(\mathbf{t}+2 q_{0}\right), \\
\mathbf{t} \in \mathbb{N}, \quad \mathbf{t}-\left[\frac{1}{\lambda}\left(K_{0}-\ln \varepsilon\right)\right] \in\left\{1,2, \ldots, 2 q_{0}\right\}, \quad \widehat{\zeta} \in \mathbb{T}^{1} .
\end{gathered}
$$

Таким образом, если $\Delta$ удовлетворяет хорошим арифметическим свойствам, то для некоторого $\mathbf{q} \in \mathbb{N}$ точки $\zeta_{s}, \ldots, \zeta_{s+\mathbf{q}-1}$ более или менее равномерно покрывают окружность $\{\zeta \bmod 1\}$. Следовательно,

$$
\mu_{s+\mathbf{q}+1}-\mu_{s+1} \approx-\sqrt{\varepsilon} \sum_{k=0}^{\mathbf{q}-1} \widehat{F}_{-}\left(\sqrt{\varepsilon} \mu_{s}, \zeta_{s}+k \Delta\right)-\sqrt{\varepsilon} \sum_{k=0}^{\mathbf{q}-1} \alpha_{s+k}
$$

Здесь первая сумма приблизительно равна $-\mathbf{q} \sqrt{\varepsilon}\left\langle\widehat{F}_{-}(0)\right\rangle$, а вторая не превосходит $2 \mathbf{q} \sqrt{\varepsilon} d$. Поэтому если $\varepsilon$ достаточно мало, то $\mu_{s+\mathbf{q}+1}-\mu_{s+1}>\frac{1}{2}\left\langle\widehat{F}_{-}(0)\right\rangle \sqrt{\varepsilon} \mathbf{q}$, так как $d$ можно считать меньшим, чем $\frac{1}{4}\left\langle\widehat{F}_{-}(0)\right\rangle$.

Точнее, мы выбираем $\mathbf{t}$ таким, что выполнены условия $(11.46),(11.47)$ и $\Delta$ удовлетворяет следующему резонансному условию $\mathbf{P У . ~}$

РУ. Пусть $C>0$ и с >0 - соответственно большая и маленъкая постоянная. Для любого $\mu_{s}$ удовлетворяющего (11.42) существуют $\mathbf{p}, \mathbf{q} \in \mathbb{Z}$ такие, что

(a) $C \leqslant \mathbf{q} \leqslant c\left(\varepsilon \ln ^{2} \varepsilon\right)^{-1 / 6}$, 
(b) дробъ $\mathbf{p} / \mathbf{q}$ несократима,

(c) существует $\Delta$, удовлетворяющее условиям (11.46), (11.47) и неравенству $|\mathbf{q} \Delta-\mathbf{p}|<\mathbf{q}^{-1}$.

ПРЕДЛОЖЕНИЕ 11.26. Для любого $\mu=\mu_{s}$, удовлетворяющего (11.42) существует $\mathbf{t}$, удовлетворяющее (11.47) такое, что для соответствующего $\Delta$ выполнено РУ.

Доказательство предложения 11.26 опустим (см. [66]).

Выберем последовательность $\mathbf{t}_{s+k}$ так, что для любого $k=0,1, \ldots, \mathbf{q}$

$$
\begin{aligned}
& \mathbf{t} \leqslant \mathbf{t}_{s+k} \leqslant \mathbf{t}+2 q_{0}, \\
& \left|\zeta_{s+k-1}+\nu\left(\sqrt{\varepsilon} \mu_{s}\right) \mathbf{t}_{s+k}-\widehat{\zeta}-k \Delta\right| \leqslant c^{-1} k^{2} \sqrt{\varepsilon}|\ln \varepsilon|, \\
& \widehat{\zeta}=\zeta_{s-1}+\nu\left(\sqrt{\varepsilon} \mu_{s}\right) \mathbf{t}_{s} .
\end{aligned}
$$

Эти неравенства можно удовлетворить. Действительно, при $k=0$ (11.48) выполнены. Далее действуем по индукции. Предположим, что при некотором $k>0$ выполнено (11.48). Тогда ввиду (11.44)

$$
\mu_{s+k}=\mu_{s}+O(k \sqrt{\varepsilon}) .
$$

Используя (11.49) и (11.45), получаем:

$$
\zeta_{s+k}+\nu\left(\sqrt{\varepsilon} \mu_{s}\right) \mathbf{t}_{s+k+1}=\zeta_{s+k-1}+\nu\left(\sqrt{\varepsilon} \mu_{s}\right)\left(t_{s+k}+\mathbf{t}_{s+k+1}\right)+\psi_{k},
$$

где $\left|\psi_{k}\right|<c^{-1} k \sqrt{\varepsilon}|\ln \varepsilon|$ и постоянная $c>0$ не зависит от $k$. Величина $(11.50)$ равна

$$
\zeta_{s+k-1}+\nu\left(\sqrt{\varepsilon} \mu_{s}\right) \mathbf{t}_{s+k}+\nu\left(\sqrt{\varepsilon} \mu_{s}\right)\left(t_{s+k}-\mathbf{t}_{s+k}+\mathbf{t}_{s+k+1}\right)+\psi_{k} .
$$

Так как $t_{s+k}-\mathbf{t}_{s+k} \in\left[0,2 q_{0}\right] \cap \mathbb{Z}$, то $\mathbf{t}_{s+k+1} \in\left[\mathbf{t}, \mathbf{t}+2 q_{0}\right] \cap \mathbb{Z}$ можно выбрать так, что $t_{s+k}-\mathbf{t}_{s+k}+\mathbf{t}_{s+k+1}=\mathbf{t}+2 q_{0}$. Это завершает индукцию.

ПреДЛОЖЕНИЕ 11.27. Пусть $\mathbf{t}$ - таково, что выполнены условия (11.46), (11.47) и соответствующее $\Delta$ удовлетворяет РУ. Предположим, что последовательность $\left(\mu_{n}, \zeta_{n}\right)$ удовлетворяет $(11.44),(11.45)$ и (11.48). Тогда

$$
\Sigma \equiv-\sum_{k=0}^{\mathbf{q}-1} \widehat{F}_{-}\left(\sqrt{\varepsilon} \mu_{s+k}, \zeta_{s+k}+\nu\left(\sqrt{\varepsilon} \mu_{s+k}\right) \mathbf{t}_{s+k+1}\right)>\frac{2 \mathbf{q}}{3}\left|\left\langle\widehat{F}_{-}(0)\right\rangle\right| .
$$

ДОКАЗАТЕЛЬСТВО ПРЕДЛОЖЕНИЯ 11.27. По определению (см. §5), функция $\widehat{F}_{-}(\eta, \zeta) \equiv \widehat{F}_{c^{\prime}, c^{\prime \prime},-}(\eta, \zeta)$ гладкая по $\eta$ при условии, что $\eta$ близко к нулю.

Используя оценку (11.49), получаем:

$$
\begin{aligned}
\Sigma & =\Sigma_{1}+O\left(\mathbf{q}^{2} \sqrt{\varepsilon}\right) \\
\Sigma_{1} & =-\sum_{k=0}^{\mathbf{q}-1} \widehat{F}_{-}\left(\sqrt{\varepsilon} \mu_{s}, \zeta_{s+k}+\nu\left(\sqrt{\varepsilon} \mu_{s+k}\right) \mathbf{t}_{s+k+1}\right) .
\end{aligned}
$$


Из оценок (11.48), (11.49) следует, что

$$
\zeta_{s+k}+\nu\left(\sqrt{\varepsilon} \mu_{s+k}\right) \mathbf{t}_{s+k+1}=\widehat{\zeta}+(k+1) \Delta+\delta_{k}, \quad\left|\delta_{k}\right|<2 c^{-1} k^{2} \sqrt{\varepsilon}|\ln \varepsilon| .
$$

Следовательно, согласно $\mathbf{P У}, \Sigma_{1}$ можно считать интегральной суммой для

$$
\int_{0}^{1} \widehat{F}_{-}\left(\sqrt{\varepsilon} \mu_{s}, \zeta\right) d \zeta=\left\langle\widehat{F}_{-}(0)\right\rangle+O\left(\sqrt{\varepsilon} \mu_{s}\right) .
$$

Если $\mathbf{q}$ достаточно велико (чтобы иметь хорошую аппроксимацию интеграла), но меньше, чем $c\left(\varepsilon \ln ^{2} \varepsilon\right)^{-1 / 6}$ (чтобы иметь $\left|\delta_{k}\right|<1 / \mathbf{q}$ ), выполнена следующая оценка:

$$
\left|\Sigma_{1}-\mathbf{q}\right|\left\langle\widehat{F}_{-}(0)\right\rangle||<\frac{1}{4} \mathbf{q}|\langle\widehat{F}(0)\rangle| .
$$

В сочетании с (11.52) это дает (11.51).

Согласно (11.44)

$$
\mu_{s+\mathbf{q}+1}-\mu_{s+1}=\sqrt{\varepsilon} \Sigma+\sqrt{\varepsilon} \sum_{k=0}^{\mathbf{q}-1} \widehat{\alpha}_{s+k+1}, \quad\left|\widehat{\alpha}_{n}\right|<2 d .
$$

Из предложения 11.27 следует, что

$$
\mu_{s+\mathbf{q}+1}-\mu_{s+1}>\sqrt{\varepsilon} \mathbf{q}\left(\frac{2}{3}\left|\left\langle\widehat{F}_{-}(0)\right\rangle\right|-2 d\right)>\frac{\sqrt{\varepsilon} \mathbf{q}}{2}\left|\left\langle\widehat{F}_{-}(0)\right\rangle\right|
$$

при условии $d<\frac{1}{12}\left\langle\widehat{F}_{-}(0)\right\rangle$. Мы продолжаем эту процедуру, пока выполнено (11.42).

7.2. Шаг 2 (около резонанса). Рассмотрим случай

$$
\widehat{C} \sqrt{\varepsilon} /|\ln \varepsilon|<|\sqrt{\varepsilon} \mu|<\varepsilon^{1 / 6} .
$$

Будем использовать равенство

$$
\nu(\sqrt{\varepsilon} \mu)=\nu_{0}+\nu_{0}^{\prime} \sqrt{\varepsilon} \mu+O\left(\varepsilon \mu^{2}\right) .
$$

Ввиду (11.53) функция $\mathbf{H}(\sqrt{\varepsilon} \mu, \zeta)$ близка к $1 / q_{0}$-периодичной по $\zeta$ функции $\widetilde{\mathbf{H}}$ (см. следствие 11.2):

$$
|\mathbf{H}(\sqrt{\mu}, \zeta)-\widetilde{\mathbf{H}}(\sqrt{\mu}, \zeta)|=O\left(\varepsilon^{j / 6} q_{0}^{j+1}\right) .
$$

Заменим Н в (11.34) на $\widetilde{\mathbf{H}}$. Тогда порядок "ошибки" $\alpha_{n}$ остается прежним.

Так как $\nu_{0}=p_{0} / q_{0}$, функции $\widehat{F}_{ \pm}(0, \zeta)-\frac{1}{q_{0}}$-периодичны по $\zeta$. Будем считать в $(11.34),(11.35)$, что $\zeta=\zeta \bmod \frac{1}{q_{0}}$. Таким образом, можно заменить $(11.35)$ равенством $\zeta_{n+1}-\zeta_{n}=\left(\nu\left(\sqrt{\varepsilon} \mu_{n}\right)-\nu_{0}\right) t_{n+1}+\beta_{n}$. Равенства (11.34), (11.35) принимают вид

$$
\begin{aligned}
\mu_{n+1}-\mu_{n} & =-\sqrt{\varepsilon}\left(\widehat{v}_{n}+t_{n+1} \widetilde{\mathbf{H}}_{\zeta}\left(\sqrt{\varepsilon} \mu_{n}, \zeta_{n}\right)+\widehat{\alpha}_{n}\right), \\
\zeta_{n+1}-\zeta_{n} & =\nu_{0}^{\prime} \sqrt{\varepsilon} \mu_{n} t_{n+1}+\beta_{n}^{\prime}+\beta_{n}^{\prime \prime}
\end{aligned}
$$




$$
\left|\widehat{\alpha}_{n}\right|<2 d, \quad\left|\beta_{n}^{\prime}\right| \leqslant C \varepsilon \mu_{n}^{2}|\ln \varepsilon|, \quad\left|\beta_{n}^{\prime \prime}\right| \leqslant C \varepsilon^{3 / 4}|\ln \varepsilon|,
$$

где $\widehat{v}_{n}$ равно одной из следующих двух величин:

$$
\widehat{F}_{ \pm}\left(0, \widehat{\zeta}_{n}\right), \quad \widehat{\zeta}_{n}=\zeta_{n-1}+\nu_{0}^{\prime} \sqrt{\varepsilon} \mu_{n-1} \mathbf{t}_{n}+O\left(\varepsilon \mu_{n-1}^{2}|\ln \varepsilon|\right) .
$$

Отметим, что ввиду (11.55), (11.56)

$$
\zeta_{n}=\widehat{\zeta}_{n}+O\left(\sqrt{\varepsilon} \mu_{n}\right)+O\left(\varepsilon^{3 / 4}|\ln \varepsilon|\right) .
$$

Возьмем

$$
\mathbf{t}=\left[\frac{1}{\lambda}\left(K_{0}-\ln \varepsilon\right)\right]+1, \quad \mathbf{t}_{*}=\mathbf{t}+2 q_{0},
$$

и последовательность $\mathbf{t}_{s+k}, k=0,1,2, \ldots$, такую, что

$$
\begin{aligned}
& \mathbf{t} \leqslant \mathbf{t}_{s+k} \leqslant \mathbf{t}+2 q_{0} \\
& \widehat{\zeta}_{s+k+1}=\widehat{\zeta}_{s+k}+\nu_{0}^{\prime} \sqrt{\varepsilon} \mu_{s+k} \mathbf{t}_{*}+\delta_{s+k}, \\
& \left|\delta_{s+k}\right|<C_{1}\left(\varepsilon \mu_{s+k}^{2}|\ln \varepsilon|+\varepsilon^{3 / 4}|\ln \varepsilon|\right) .
\end{aligned}
$$

Последовательность $\mathbf{t}_{s+k}$ можно выбрать следующим образом. Ввиду (11.56) и $(11.55)$

$$
\begin{aligned}
\widehat{\zeta}_{s+k+1}= & \zeta_{s+k}+\nu_{0}^{\prime} \sqrt{\varepsilon} \mu_{s+k} \mathbf{t}_{s+k+1}+O\left(\varepsilon \mu_{s+k}^{2}|\ln \varepsilon|\right) \\
= & \zeta_{s+k-1}+\nu_{0}^{\prime} \sqrt{\varepsilon}\left(\mu_{s+k-1} t_{s+k}+\mu_{s+k} \mathbf{t}_{s+k+1}\right) \\
& \quad+\beta_{s+k-1}^{\prime}+\beta_{s+k-1}^{\prime \prime}+O\left(\varepsilon \mu_{s+k}^{2}|\ln \varepsilon|\right) \\
= & \widehat{\zeta}_{s+k}+\nu_{0}^{\prime} \sqrt{\varepsilon}\left(\mu_{s+k-1} t_{s+k}-\mu_{s+k-1} \mathbf{t}_{s+k}+\mu_{s+k} \mathbf{t}_{s+k+1}\right) \\
& +\beta_{s+k-1}^{\prime}+\beta_{s+k-1}^{\prime \prime}+O\left(\varepsilon \mu_{s+k}^{2}|\ln \varepsilon|\right) .
\end{aligned}
$$

Возьмем $\mathbf{t}_{s+k+1} \in\left[\mathbf{t}, \mathbf{t}+2 q_{0}\right] \cap \mathbb{Z}$ таким, что $t_{s+k}-\mathbf{t}_{s+k}+\mathbf{t}_{s+k+1}=\mathbf{t}+2 q_{0}$. Тогда выполняется (11.58).

Лемма 11.23 на шаге 2 вытекает из следующего предложения.

ПРЕДЛОЖЕНИЕ 11.28. Для любъх

$$
\mu_{s}, \zeta_{s}, \quad \text { дde } \quad \sqrt{\varepsilon} \mu_{s}>-\varepsilon^{1 / 6}, \quad \zeta_{s}=\zeta_{s} \bmod \frac{1}{q_{0}},
$$

существует траектория

$$
\left(\mu_{n}, \zeta_{n}\right), \quad n=s, \ldots, s+N
$$

задачи 2 такая, что

(a) $\mu_{s+N}>-\widehat{C} /|\ln \varepsilon|$,

(b) $N<C \varepsilon^{-5 / 6}$.

Используя предложение 11.28 , можно пройти через интервал $-\varepsilon^{1 / 6} \leqslant \sqrt{\varepsilon} \mu \leqslant$ $-\widehat{C} \sqrt{\varepsilon} /|\ln \varepsilon|$. Интервал $\widehat{C} \sqrt{\varepsilon} /|\ln \varepsilon| \leqslant \sqrt{\varepsilon} \mu \leqslant \varepsilon^{1 / 6}$ проходится аналогично. 
Чтобы построить траекторию 11.59 , рассмотрим систему

$$
\begin{gathered}
\left\{\begin{array}{l}
\dot{\mu}=-\frac{1}{\mathbf{t}_{*}}\left(\mathscr{F}_{ \pm}\right)_{\zeta}(\zeta)-\widetilde{\mathbf{H}}_{\zeta}(\sqrt{\varepsilon} \mu, \zeta), \\
\dot{\zeta}=\nu_{0}^{\prime} \mu+\sqrt{\varepsilon} \widetilde{\mathbf{H}}_{\eta}(\sqrt{\varepsilon} \mu, \zeta),
\end{array}\right. \\
\left(\mathscr{F}_{ \pm}\right)_{\zeta}(\zeta)=\widehat{F}_{ \pm}(0, \zeta)-\mathbf{F}_{ \pm}, \quad \mathbf{F}_{ \pm}=\int_{0}^{1} \widehat{F}_{ \pm}(0, \zeta) d \zeta .
\end{gathered}
$$

Эта система гамильтонова с симплектической структурой $d \mu \wedge d \zeta$ и гамильтонианом

$$
\begin{aligned}
\chi_{ \pm}(\mu, \zeta) & =\frac{1}{2} \nu_{0}^{\prime} \mu^{2}+\widetilde{\mathbf{H}}(\sqrt{\varepsilon} \mu, \zeta)+\frac{1}{\mathbf{t}_{*}} \mathscr{F}_{ \pm}(\zeta)+h_{ \pm}, \\
h_{ \pm} & =-\max _{\zeta}\left(\widetilde{\mathbf{H}}(\sqrt{\varepsilon} \mu, \zeta)+\frac{1}{\mathbf{t}_{*}} \mathscr{F}_{ \pm}(\zeta)\right) .
\end{aligned}
$$

Далее в этом параграфе мы изучим свойства системы с гамильтонианом $\chi_{-}$. Случай гамильтониана $\chi_{+}$рассматривается аналогично. Отметим, что ввиду (11.54), (11.55)

$$
\begin{aligned}
& \chi_{-}\left(\mu_{n+1}, \zeta_{n+1}\right)-\chi_{-}\left(\mu_{n}, \zeta_{n}\right)=\boldsymbol{d}_{n}^{ \pm}-\sqrt{\varepsilon} \nu_{0}^{\prime} \mu_{n} \widehat{\alpha}_{n}+\widetilde{\beta}_{n}^{\prime}+\widetilde{\beta}_{n}^{\prime \prime}, \\
& \boldsymbol{d}_{n}^{ \pm}=-\sqrt{\varepsilon} \nu_{0}^{\prime} \mu_{n} \widehat{F}_{ \pm}\left(0, \widehat{\zeta}_{n}\right)+\frac{1}{\mathbf{t}_{*}}\left(\mathscr{F}_{-}\left(\zeta_{n+1}\right)-\mathscr{F}_{-}\left(\zeta_{n}\right)\right), \\
& \left|\widetilde{\beta}_{n}^{\prime}\right| \leqslant \widetilde{C} \varepsilon \mu_{n}^{2}|\ln \varepsilon|, \quad\left|\widetilde{\beta}_{n}^{\prime \prime}\right| \leqslant \widetilde{C} \varepsilon^{3 / 4}|\ln \varepsilon| .
\end{aligned}
$$

Индекс + или - в $\boldsymbol{d}_{n}^{ \pm}$соответствует выбору $\widehat{v}_{n}$ в (11.56).

ЗАмечАниЕ 11.29. Согласно (11.58), разность $\widehat{\zeta}_{n+1}-\widehat{\zeta}_{n} \in \mathbb{R} /\left(q_{0}^{-1} \mathbb{Z}\right)$ близка к нулю. Далее мы считаем $\widehat{\zeta}_{n+1}-\widehat{\zeta}_{n}$ (соответственно $\left.\zeta_{n+1}-\zeta_{n}\right)$ вещественным числом, лежащим в малой окрестности нуля. Ввиду этого соглашения мы считаем $\widehat{\zeta}_{s+k+1}-\widehat{\zeta}_{s}\left(\right.$ соответственно $\left.\zeta_{s+k+1}-\zeta_{s}\right)$ суммой $\sum_{n=s}^{s+k}\left(\widehat{\zeta}_{n+1}-\widehat{\zeta}_{n}\right)$ (соответственно $\left.\sum_{n=s}^{s+k}\left(\zeta_{n+1}-\zeta_{n}\right)\right)$.

Для $s \leqslant k^{\prime} \leqslant k^{\prime \prime}$ положим $\mathbf{D}_{k^{\prime}, k^{\prime \prime}}^{ \pm}=\chi_{-}\left(\mu_{k^{\prime \prime}}, \zeta_{k^{\prime \prime}}\right)-\chi_{-}\left(\mu_{k^{\prime}}, \zeta_{k^{\prime}}\right)$, где верхний индекс у $\mathbf{D}$ тот же, что и у $\boldsymbol{d}_{n}^{ \pm}$в (11.64).

ПреДЛОЖенИЕ 11.30. Пусть последовательность $\zeta_{k^{\prime}}, \zeta_{k^{\prime}+1}, \ldots, \zeta_{k^{\prime \prime}}$ монотонна. Тогда существует постоянная $K \in \mathbb{N}$ такая, что при $k^{\prime \prime}-k^{\prime} \geqslant K u$ npu $\left|\widehat{\zeta}_{s+k+1}-\widehat{\zeta}_{s}\right|<1$

$$
\mathbf{D}_{k^{\prime}, k^{\prime \prime}}^{ \pm}=-\frac{1}{\mathbf{t}_{*}} a_{ \pm}+a_{ \pm}^{\prime}+a_{ \pm}^{\prime \prime}
$$

əəe

$$
\begin{aligned}
a_{ \pm} & =-\int_{\zeta_{k^{\prime}}}^{\zeta_{k^{\prime \prime}}} \widehat{F}_{ \pm}(0, \zeta) d \zeta+\mathscr{F}\left(\zeta_{k^{\prime \prime}}\right)-\mathscr{F}\left(\zeta_{k^{\prime}}\right) \\
\left|a_{ \pm}^{\prime}\right| & <\frac{2 d}{\mathbf{t}_{*}}\left|\zeta_{k^{\prime \prime}}-\zeta_{k^{\prime}}\right|, \\
\left|a_{ \pm}^{\prime \prime}\right| & <C\left(\sqrt{\varepsilon} \mu_{k^{\prime}, k^{\prime \prime}}+\left(k^{\prime \prime}-k^{\prime}\right) \varepsilon \mu_{k^{\prime}, k^{\prime \prime}}^{2}|\ln \varepsilon|+\left(k^{\prime \prime}-k^{\prime}\right) \varepsilon^{3 / 4}|\ln \varepsilon|\right), \\
\mu_{k^{\prime}, k^{\prime \prime}} & =\max _{k^{\prime} \leqslant n \leqslant k^{\prime \prime}}\left|\mu_{n}\right| .
\end{aligned}
$$


Доказательство предложения 11.30 содержится в $\S 9$.

Отметим, что, согласно (11.61) и (11.66),

$$
a_{-}=-\left(\zeta_{k^{\prime \prime}}-\zeta_{k^{\prime}}\right) \mathbf{F}_{-}, \quad a_{-}-a_{+}=\int_{\zeta_{k^{\prime}}}^{\zeta_{k^{\prime \prime}}}\left(\widehat{F}_{+}(0, \zeta)-\widehat{F}_{-}(0, \zeta)\right) d \zeta
$$

Траектория (11.59) строится следующим образом. Возьмем в (11.54) $\widehat{v}_{n}=$ $\widehat{F}_{-}\left(0, \widehat{\zeta}_{n}\right)$. Рассмотрим постоянную $K$, удовлетворяющую предложению 11.30 . При $K \leqslant n-s \leqslant c \frac{\mu_{n}}{\sqrt{\varepsilon}|\ln \varepsilon|}$

$$
\left|\mu_{s}-\mu_{n}\right|=|O(K \sqrt{\varepsilon} \ln \varepsilon)|<\mu_{n} / 2 .
$$

Разность $\zeta_{n}-\zeta_{n-K}$ отрицательна, так как в (11.55) $\mu_{n}<0$. Более того, ввиду (11.68)

$$
\zeta_{n}-\zeta_{n-K}<\frac{1}{3} \nu_{0}^{\prime} \sqrt{\varepsilon} \mu_{n} K \frac{|\ln \varepsilon|}{\lambda} .
$$

Согласно предложению 11.30: $\chi_{-}\left(\mu_{n}, \zeta_{n}\right)-\chi_{-}\left(\mu_{n-K}, \zeta_{n-K}\right)=\mathbf{D}_{n-K, n}^{-}$,

$$
\begin{aligned}
\mathbf{D}_{n-K, n}^{-} & <-\frac{\left(\zeta_{n}-\zeta_{n-K}\right) \mathbf{F}_{-}}{\mathbf{t}_{*}}+\frac{2 d\left|\zeta_{n}-\zeta_{n-K}\right|}{\mathbf{t}_{*}}+2 C \sqrt{\varepsilon} \mu_{n}+2 C K \varepsilon^{3 / 4}|\ln \varepsilon| \\
& <-\frac{\mathbf{F}_{-}+2 d}{4} \sqrt{\varepsilon} \mu_{n} \nu_{0}^{\prime} K
\end{aligned}
$$

при условии, что постоянная $K$ достаточно велика по сравнению с $C$. Напомним, что согласно $\mathbf{H}_{\mathbf{1}} \mathbf{2}$ имеем: $\mathbf{F}_{-}=0$. Поэтому, действуя таким образом, мы достигнем точки $\left(\mu_{n_{0}}, \zeta_{n_{0}}\right)$ такой, что

$$
\mu_{n_{0}-1} \leqslant-\widehat{C} /|\ln \varepsilon| \leqslant \mu_{n_{0}} .
$$

Более того, поскольку на этой части траектории среднее приращение $\mu$ имеет порядок $\sqrt{\varepsilon}$, получаем оценку

$$
n_{0}-s \leqslant C \varepsilon^{-5 / 6} .
$$

Построение траектории для $\mu>\widehat{C} /|\ln \varepsilon|, \chi_{+}(\mu, \zeta)>0$ аналогично: при $\widehat{v}=$ $\widehat{F}_{+}(0, \widehat{\zeta})$ энергия $\chi_{+}$растет.

7.3. Шаг 3 (в резонансе). Покажем, как провести траекторию через цилиндр

$$
\mathbf{S}=\{(\mu, \zeta):|\mu| \leqslant \widehat{C} /|\ln \varepsilon|\} .
$$

Ввиду (11.63) и $\mathbf{H}_{\mathbf{1}} \mathbf{3}$ уравнение $\chi=0$ задает сепаратрисы. Эти сепаратрисы являются внешними в том смысле, что все критические точки $\chi$ - расположены в области $\left\{\chi_{-} \leqslant 0\right\}$. Возможно, имеются также внутренние сепаратрисы, соответствующие меньшим значениям энергии, но далее мы будем говорить только о внешних сепаратрисах. Кривую $\{\chi=0, \mu<0\}$ назовем нижней сепаратрисой, a $\{\chi=0, \mu<0\}-$ верхней.

Согласно $\mathbf{H}_{\mathbf{1}} \mathbf{3}$, при достаточно малых $\varepsilon$ максимум (11.63) достигается в единственной точке $\zeta_{ \pm}=\zeta_{ \pm}(\varepsilon)=\zeta_{*}+O(1 / \ln \varepsilon)$. 
Рассмотрим прямоугольник

$$
\Pi=\left\{\left(\mu, \zeta \bmod 1 / q^{0}\right):|\mu| \leqslant \widehat{C} /|\ln \varepsilon|,\left|\zeta-\zeta_{*}\right| \leqslant \widehat{C}_{1} /|\ln \varepsilon|\right\},
$$

где постоянные $\widehat{C}_{1}, \widehat{C}$ таковы, что при малых $\varepsilon$

1) энергия $\chi$ - отрицательна в четырех точках

$$
(\mu, \zeta)=\left( \pm \widehat{C} /|\ln \varepsilon|, \zeta_{*} \pm \widehat{C}_{1} /|\ln \varepsilon|\right)
$$

(см. рис. 11.1);

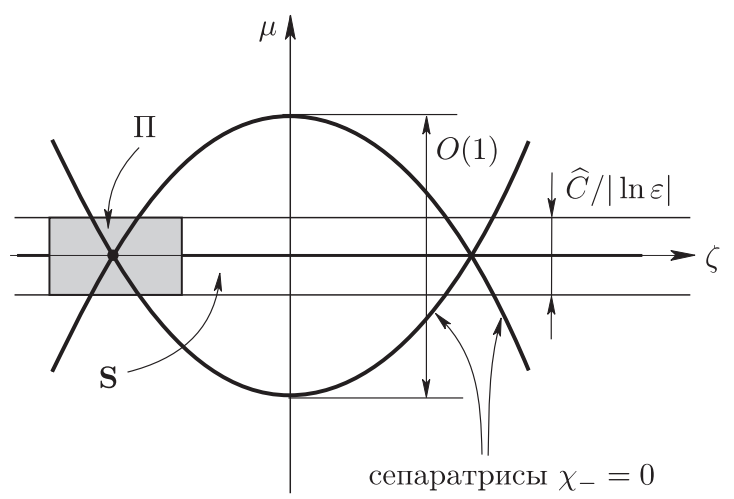

Рис. 11.1

2) $\left|\zeta_{ \pm}(\varepsilon)-\zeta_{*}\right|<\widehat{C}_{1} / 2$.

В п. 7.2 мы продолжили траекторию задачи 2 до точки $\left(\mu_{n_{0}}, \zeta_{n_{0}}\right) \in \mathbf{S}$, $\mu_{n_{0}}=-\widehat{C} /|\ln \varepsilon|+O(\sqrt{\varepsilon} \ln \varepsilon)$. Возможны два случая. Точка $\left(\mu_{n_{0}}, \zeta_{n_{0}}\right)$ может оказаться вне и внутри П. В первом случае $\chi\left(\mu_{n_{0}}, \zeta_{n_{0}}\right)<0$. Следовательно, траектория уже пересекла нижнюю сепаратрису. Во втором случае управление траекторией может быть потеряно. Чтобы избежать этого, вернемся немного назад. Точнее, стартуя с некоторой точки $\left(\mu_{n^{\prime}}, \zeta_{n^{\prime}}\right), n^{\prime}<n_{0}$, мы будем строить траекторию как на шаге 2 , используя $\widehat{v}=\widehat{f}_{+}(0, \widehat{\zeta})$ вместо $\widehat{v}=\widehat{f}_{-}(0, \widehat{\zeta})$.

Обозначим новую траекторию

$$
\left(\mu_{n^{\prime}}, \zeta_{n^{\prime}}\right),\left(\mu_{n^{\prime}+1}^{+}, \zeta_{n^{\prime}+1}^{+}\right),\left(\mu_{n^{\prime}+2}^{+}, \zeta_{n^{\prime}+2}^{+}\right), \ldots,\left(\mu_{n^{\prime \prime}}^{+}, \zeta_{n^{\prime \prime}}^{+}\right)
$$

Теперь, согласно предложению 11.30, энергия $\chi$ на траектории уменьшается медленнее или вообще не уменьшается. Выберем $\left(\mu_{n^{\prime}}, \zeta_{n^{\prime}}\right)$ так, что

$$
\int_{\zeta_{n_{0}}}^{\zeta_{n^{\prime}}}\left(\widehat{F}_{+}(0, \zeta)-\widehat{F}_{-}(0, \zeta)\right) d \zeta=c_{0} /|\ln \varepsilon|+O(\sqrt{\varepsilon} \ln \varepsilon)
$$

Затем возьмем точку $\left(\mu_{n^{\prime \prime}}^{+}, \zeta_{n^{\prime \prime}}^{+}\right), n^{\prime \prime}>n^{\prime}$, такую, что $\zeta_{n^{\prime \prime}}^{+}=\zeta_{n_{0}}+O(\sqrt{\varepsilon} \ln \varepsilon)$. Ввиду предложения 11.30,

$$
\chi_{-}\left(\mu_{n^{\prime \prime}}^{+}, \zeta_{n^{\prime \prime}}^{+}\right)-\chi_{-}\left(\mu_{n_{0}}, \zeta_{n_{0}}\right)=\frac{c_{0}+Y_{1}}{\mathbf{t}_{*}|\ln \varepsilon|},
$$


где $\left|Y_{1}\right|<3 d$ при достаточно малых $\varepsilon$. Следовательно,

$$
\begin{gathered}
\frac{1}{2} \nu_{0}^{\prime}\left(\mu_{n^{\prime \prime}}^{+}\right)^{2}-\frac{1}{2} \nu_{0}^{\prime} \mu_{n_{0}}^{2}+O(\sqrt{\varepsilon} \ln \varepsilon)=\frac{c_{0}+Y_{1}}{\mathbf{t}_{*}|\ln \varepsilon|}, \\
\left(\mu_{n^{\prime \prime}}^{+}\right)^{2}=\mu_{n_{0}}^{2}+\frac{2\left(c_{0}+Y_{2}\right)}{\nu_{0}^{\prime} \mathbf{t}_{*}|\ln \varepsilon|}, \quad\left|Y_{2}\right|<4 d .
\end{gathered}
$$

(Напомним, что $\mu_{n^{\prime \prime}}^{+}$и $\mu_{n_{0}}$ отрицательны.)

При некотором $c_{0}$ продолжение траектории $(11.73)$ с $\widehat{v}_{n}=\widehat{F}_{-}\left(0, \widehat{\zeta}_{n}\right)$ пересечет нижнюю сепаратрису вне П. Поэтому, переобозначая $\left(\mu_{n}^{+}, \zeta_{n}^{+}\right)$на $\left(\mu_{n}, \zeta_{n}\right)$ и $n^{\prime \prime}$ на $n_{0}$, мы возвращаемся к предположению, что (11.71) справедливо и $\left(\mu_{n_{0}}, \zeta_{n_{0}}\right) \in \mathbf{S} \backslash \Pi$.

Теперь мы собираемся перейти через резонанс и пересечь верхнюю сепаратрису вне П. Рассмотрим значение энергии $\chi_{+}\left(\mu_{n_{0}}, \zeta_{n_{0}}\right)$ (вместо $\left.\chi_{-}\right)$. Имеется два случая.

1) $\chi_{+}\left(\mu_{n_{0}}, \zeta_{n_{0}}\right)>-c_{\bullet} /|\ln \varepsilon|$, где $c_{\bullet}>0$ достаточно мало.

В этом случае мы пройдем $\mathbf{S}$, используя $\widehat{v}_{n}=\widehat{F}_{-}\left(0, \widehat{\zeta}_{n}\right)$ (это возможно, так как $\left(\mu_{n_{0}}, \zeta_{n_{0}}\right)$ находится вне П). При этом понадобится $O\left(\varepsilon^{-1 / 2}|\ln \varepsilon|^{-2}\right)$ шагов, и $\zeta$ изменится на величину $\sim \ln ^{-2} \varepsilon$. Таким образом, траектория покинет $\mathbf{S}$ с

$$
\chi_{+}\left(\mu_{n_{0}}, \zeta_{n_{0}}\right)>-c_{\bullet} /|\ln \varepsilon|+O\left(\ln ^{-2} \varepsilon\right) .
$$

Тогда мы переключимся на $\widehat{v}_{n}=\widehat{F}_{+}\left(0, \widehat{\zeta}_{n}\right)$. Согласно (11.74) траектория пересечет верхнюю сепаратрису вне П.

2) $\chi_{+}\left(\mu_{n_{0}}, \zeta_{n_{0}}\right)<-c_{\bullet} /|\ln \varepsilon|$. В этом случае мы вернемся назад. Используя управление $\widehat{v}_{n}=\widehat{F}_{+}\left(0, \widehat{\zeta}_{n}\right)$, начиная с некоторого $n^{\prime}<n_{0}$, мы получим траекторию, на которой $\chi_{+}$окажется больше. Выбирая $n^{\prime}$ и $n^{\prime \prime}>n^{\prime}$, где опять $\widehat{v}_{n}=\widehat{F}_{+}\left(0, \widehat{\zeta}_{n}\right)$, мы сводим ситуацию к случаю $(1)$.

\section{§ 8. Доказательство леммы 11.24}

С помощью леммы 11.22 можно построить траекторию такую, что на соответствующей последовательности $\left(\bar{\Omega}_{k}, \bar{\Omega}_{k+1}\right),\left(\bar{\Omega}_{k+1}, \bar{\Omega}_{k+2}\right), \ldots$ правых совместных квазитраекторий в соответствии с нашими нуждами $v_{n}=\widehat{\Theta}_{\zeta}\left(\varepsilon \bar{\rho}_{n}, \bar{\zeta}_{n}, \bar{\tau}_{n}\right)$, $n=k, k+1, \ldots$, равно

$$
\text { либо } \widehat{F}_{+}^{\sigma_{n}}\left(\varepsilon \rho_{n-1}, \zeta_{n-1}+\nu\left(\varepsilon \rho_{n-1}\right) \mathbf{t}_{n}\right), \quad \text { либо } \quad \widehat{F}_{-}^{\sigma_{n}}\left(\varepsilon \rho_{n-1}, \zeta_{n-1}+\nu\left(\varepsilon \rho_{n-1}\right) \mathbf{t}_{n}\right)
$$

(см. (11.39)).

Необходимо показать, что коды $\left(\bar{\Omega}_{k}, \bar{\Omega}_{k+1}\right)$ можно выбрать так, что на соответствующей траектории $\varepsilon \rho$ проходит через интервал I. Для этого мы сведем задачу к задаче 2. Действительно, при $\varepsilon \bar{\rho}_{n} \in \mathbf{I}$, соединяя (10.13) с оценками ${ }^{14}$

$$
\left|\bar{\rho}_{n}-\rho_{n}\right|<b / b_{\rho}, \quad\left|\bar{\zeta}_{n}-\zeta_{n}\right|<b / b_{\zeta}, \quad\left|\bar{\tau}_{n}-\tau_{n}\right|<b / b_{\tau}
$$

\footnotetext{
${ }^{14}$ Напомним, что $b_{\rho}, b_{\zeta}, b_{\tau}$ удовлетворяют $(10.25)-(10.27)$ и, в частности, величины $b / b_{\rho}$, $b / b_{\zeta}, b / b_{\tau}$ малы.
} 
получаем:

$$
\begin{aligned}
\bar{\rho}_{n+1} & =\bar{\rho}_{n}-\widehat{\Theta}_{\zeta}\left(\varepsilon \bar{\rho}_{n}, \bar{\zeta}_{n}, \bar{\tau}_{n}\right)+\left(\bar{\tau}_{n+1}-\bar{\tau}_{n}-\bar{t}_{n+1}\right) \mathbf{H}_{\zeta}\left(\varepsilon \bar{\rho}_{n}, \bar{\zeta}_{n}\right)+\widetilde{\alpha}_{n} \\
\bar{\zeta}_{n+1} & =\bar{\zeta}_{n}+\nu\left(\varepsilon \bar{\rho}_{n}\right) \bar{t}_{n+1}+\widetilde{\beta}_{n}
\end{aligned}
$$

где при малых $d>0$ имеем: $\left|\widetilde{\alpha}_{n}\right|<d / 2,\left|\widetilde{\beta}_{n}\right|<C \varepsilon^{3 / 4}|\ln \varepsilon| / 2$. Обозначим

$$
\begin{aligned}
\mu_{n} & =\sqrt{\varepsilon}\left(\bar{\rho}_{n}-\bar{\tau}_{n} \mathbf{H}_{\zeta}\left(\varepsilon \bar{\rho}_{n}, \bar{\zeta}_{n}\right)\right) \\
\alpha_{n} & =\widetilde{\alpha}_{n}+\bar{\tau}_{n+1}\left(\mathbf{H}_{\zeta}\left(\varepsilon \bar{\rho}_{n}, \bar{\zeta}_{n}\right)-\mathbf{H}_{\zeta}\left(\varepsilon \bar{\rho}_{n+1}, \bar{\zeta}_{n+1}\right)\right)
\end{aligned}
$$

В результате получаем (11.34). Так как $\nu\left(\varepsilon \bar{\rho}_{n}\right)=\nu\left(\sqrt{\varepsilon} \mu_{n}\right)+O(\varepsilon)$, получаем (11.35).

\section{§ 9. Добавление к $§ 7$}

ДокАЗАТЕЛЬСтво предложения 11.30. Имеем:

$$
\begin{gathered}
\mathbf{D}_{k^{\prime}, k^{\prime \prime}}^{ \pm}=\Sigma_{ \pm}^{\prime}+\Sigma_{ \pm}^{\prime \prime}+\Sigma_{ \pm}^{\prime \prime \prime} \\
\Sigma_{ \pm}^{\prime}=\sum_{n=k^{\prime}}^{k^{\prime \prime}-1} \boldsymbol{d}_{n}^{-}, \quad \Sigma_{ \pm}^{\prime \prime}=-\sqrt{\varepsilon} \nu_{0}^{\prime} \sum_{n=k^{\prime}}^{k^{\prime \prime}-1} \mu_{n} \widehat{\alpha}_{n}, \quad \Sigma_{ \pm}^{\prime \prime \prime}=\sum_{n=k^{\prime}}^{k^{\prime \prime}-1}\left(\beta_{n}^{\prime}+\beta_{n}^{\prime \prime}\right) .
\end{gathered}
$$

Предложение 11.30 следует из оценок

$$
\begin{aligned}
\Sigma_{ \pm}^{\prime} & =\frac{1}{\mathbf{t}_{*}}\left(-\int_{\zeta_{k^{\prime}}}^{\zeta_{k^{\prime \prime}}} \widehat{F}_{ \pm}(0, \zeta) d \zeta+\mathscr{F}\left(\zeta_{k^{\prime \prime}}\right)-\mathscr{F}\left(\zeta_{k^{\prime}}\right)+\delta^{\prime}\right) \\
\left|\delta^{\prime}\right| & <C\left(\sqrt{\varepsilon} \mu_{k^{\prime}, k^{\prime \prime}}+k \varepsilon \mu_{k^{\prime}, k^{\prime \prime}}^{2}|\ln \varepsilon|+k \varepsilon^{3 / 4}|\ln \varepsilon|\right), \\
\left|\Sigma^{\prime \prime}\right| & <\frac{2 d}{\mathbf{t}_{*}}\left|\zeta_{k^{\prime \prime}}-\zeta_{k^{\prime}}\right|+\frac{C\left(k^{\prime \prime}-k^{\prime}\right)}{\Delta}\left(\varepsilon \mu_{k^{\prime}, k^{\prime \prime}}^{2}+\varepsilon^{3 / 4}\right)|\ln \varepsilon|
\end{aligned}
$$

Сначала проверим (11.75):

$$
\Sigma_{ \pm}^{\prime}=-\sum_{n=k^{\prime}}^{k^{\prime \prime}-1} \sqrt{\varepsilon} \nu_{0}^{\prime} \mu_{n} \widehat{F}_{ \pm}\left(0, \widehat{\zeta}_{n}\right)+\frac{1}{\mathbf{t}_{*}}\left(\mathscr{F}\left(\zeta_{k^{\prime \prime}}\right)-\mathscr{F}\left(\zeta_{k^{\prime}}\right)\right)
$$

Ввиду (11.58) множитель $\sqrt{\varepsilon} \nu_{0}^{\prime} \mu_{n}$ в последней сумме равен $\frac{1}{\mathbf{t}_{*}}\left(\widehat{\zeta}_{n+1}-\widehat{\zeta}_{n}-\delta_{n}\right)$. Следовательно,

$$
\begin{aligned}
\Sigma_{ \pm}^{\prime} & =\frac{1}{\mathbf{t}_{*}}\left(\Sigma_{1 \pm}^{\prime}+\Sigma_{2 \pm}^{\prime}+\mathscr{F}\left(\zeta_{k^{\prime \prime}}\right)-\mathscr{F}\left(\zeta_{k^{\prime}}\right)\right) \\
\Sigma_{1 \pm}^{\prime} & =-\sum_{n=k^{\prime}}^{k^{\prime \prime}-1} \widehat{F}_{ \pm}\left(0, \widehat{\zeta}_{n}\right)\left(\widehat{\zeta}_{n+1}-\widehat{\zeta}_{n}\right) \\
\Sigma_{2 \pm}^{\prime} & =\sum_{n=k^{\prime}}^{k^{\prime \prime}-1} \widehat{F}_{ \pm}\left(0, \widehat{\zeta}_{n}\right) \delta_{n} .
\end{aligned}
$$


Функции $\widehat{F}_{ \pm}$кусочно гладкие. Так что, согласно (11.57), имеем:

$$
\left|\Sigma_{1 \pm}^{\prime}+\int_{\zeta_{k^{\prime}}}^{\zeta_{k^{\prime \prime}}} \widehat{F}_{ \pm}(0, \zeta) d \zeta\right|<C \sqrt{\varepsilon} \mu_{k^{\prime}, k^{\prime \prime}}
$$

Сумму $\Sigma_{2 \pm}^{\prime}$ можно оценить следующим образом:

$$
\left|\Sigma_{2 \pm}^{\prime}\right| \leqslant C_{1} \sum_{n=k^{\prime}}^{k^{\prime \prime}}\left|\delta_{n}\right| \leqslant C\left(k^{\prime \prime}-k^{\prime}\right)\left(\mu_{k^{\prime}, k^{\prime \prime}}^{2} \varepsilon+\varepsilon^{3 / 4}\right)|\ln \varepsilon| .
$$

Отсюда следует (11.75).

Чтобы проверить (11.76), заметим, что $\left|\Sigma^{\prime \prime}\right| \leqslant \frac{2 d}{\mathbf{t}_{*}}\left|\Sigma_{n=k^{\prime}}^{k^{\prime \prime}-1}\left(\widehat{\zeta}_{n+1}-\widehat{\zeta}_{n}-\delta_{n}\right)\right|$. Таким образом,

$$
\left|\Sigma_{ \pm}^{\prime \prime}\right| \leqslant \frac{2 d}{\mathbf{t}_{*}}\left|\widehat{\zeta}_{k^{\prime \prime}}-\widehat{\zeta}_{k^{\prime}}\right|+\frac{2 d}{\mathbf{t}_{*}} \sum_{n=k^{\prime}}^{k^{\prime \prime}-1}\left|\delta_{n}\right| .
$$

Последнюю сумму можно оценить по аналогии с $\Sigma_{2 \pm}^{\prime}$.

\section{§ 10. Вспомогательные утверждения}

ДОКАЗАТЕЛЬСТВо ПРЕДЛОЖЕНИЯ 11.1. Разность $\mathbf{H}\left(y^{0}, x+p / q\right)-\mathbf{H}\left(y^{0}, x\right)$ равна

$$
\sum_{\left(k, k_{0}\right) \in \mathbb{Z}^{2}} \phi\left(\frac{k p / q+k_{0}+k \delta}{\varepsilon^{1 / 4}}\right) H_{1}^{k, k_{0}}\left(y^{0}\right) e^{2 \pi i k x}\left(e^{2 \pi i k p / q}-1\right) .
$$

Рассмотрим ненулевые слагаемые в этой сумме. Сначала заметим, что

$$
\left|k p / q+k_{0}+k \delta\right|<\varepsilon^{1 / 4},
$$

иначе $\phi=0$. Далее, $k p / q \notin \mathbb{Z}$, так как иначе $e^{2 \pi i k p / q}-1=0$. Таким образом, имеем: $\left|k p / q+k_{0}\right| \geqslant 1 / q>2 \varepsilon^{1 / 4}$. Отсюда и из (11.77) следует, что $|k \delta|>1 /(2 q)$, т.е. $|k|>1 /(2 q \delta)$. Неравенство (11.1) следует из стандартной оценки $\left|H_{1}^{k, k_{0}}\right|<$ $C_{1}\left(|k|+\left|k_{0}\right|\right)^{-j}$. Предложение 11.1 доказано.

ДокАЗАТЕЛЬСтво ПРЕДЛОЖЕНИЯ 11.5. По теореме Дирихле получаем, что существует $q \leqslant K-1, p \in \mathbb{Z}$ такие, что дробь $p / q$ несократима и $|q \nu-p| \leqslant \frac{1}{K}$, т.е. $\nu=p / q+\xi,|\xi|<1 /(q K)$.

Пусть $I \subset \operatorname{pr}_{\bar{\nu}} j_{+}^{\sigma}(\eta)$. Предположим, что $q>2 /|I|$. Тогда для доказательства предложения 11.5 достаточно доказать, что

$$
\bigcup_{0 \leqslant t \leqslant K} g^{\eta, t}(I)=\mathbb{T}^{2}
$$

Равенство (11.78) вытекает из того, что

$$
\bigcup_{n=0}^{K-1} g^{\eta, n}(I)=\mathbb{T}_{0} \equiv \mathbb{T}^{2} \cap\{\tau=0\}
$$


Последнее равенство эквивалентно следующему:

$$
\mathbb{T}=\left(\bigcup_{n=0}^{K-1}[n \nu,|I|+n \nu]\right) \bmod 1 .
$$

Заменим последнее множество его подмножеством:

$$
U=\left(\bigcup_{n=0}^{q-1}\left[\frac{n p}{q}+n \xi,|I|+\frac{n p}{q}+n \xi\right]\right) \bmod 1 .
$$

Если $\xi=0, U$ накрывает по крайней мере дважды тор $\mathbb{T}$. Действительно, это следует из предположения $|I|>2 / q$. Поскольку $|n \xi|<1 / K<1 / q$ для $0<n<q-1, U$ совпадает с $\mathbb{T}_{0}$.

\section{Список литературы}

[1] Г. М. Заславский, Н.Н.Филоненко, "Стохастическая неустойчивость захваченных частиц и условия применимости квазилинейного приближения", ЖЖТФ, 54 (1968), 1590-1602; англ. пер.: G. M. Zaslavskii, N. N. Filonenko, "Stochastic instability of trapped particles and conditions of applicability of the quasi-linear approximation", Soviet Phys. JETP, 27 (1968), 851-857.

[2] Л.П. Шильников, "Об одном случае существования счетного множества периодических движений", Докл. АН СССР, 160:3 (1965), 558-561; англ. пер.: L. P. Shil'nikov, "A case of the existence of a countable number of periodic motions", Soviet Math. Dokl., 6 (1965), 163-166.

[3] J. M. Greene, "A method for determining a stochastic transition", J. Math. Phys., 20:6 (1979), 1183-1201.

[4] R. S. MacKay, I. C. Percival, "Converse KAM: Theory and practice", Comm. Math. Phys., 98:4 (1985), 469-512.

[5] A. Olvera, C. Simó, "An obstruction method for the destruction of invariant curves", Phys. D, 26:1-3 (1987), 181-192.

[6] N. N. Filonenko, R. Z. Sagdeev, G. M. Zaslavsky, "Destruction of magnetic surfaces by magnetic field irregularities. II", Nucl. Fusion, 7 (1967), 253-266.

[7] B. V. Chirikov, "A universal instability of many-dimensional oscillation systems", Phys. Rep., 52:5 (1979), 263-379.

[8] С. А. Довбыш, "Структура Колмогоровского множества возле сепаратрис плоского отображения", Матем. заметки, 46:4 (1989), 112-114.

[9] T. Ahn, G. Kim, S. Kim, "Analysis of the separatrix map in Hamiltonian systems", Phys. D, 89:3-4 (1996), 315-328.

[10] В.Ф. Лазуткин, "О ширине зоны устойчивости возле сепаратрис стандартного отображения", Докл. АН СCCP, 313:2 (1990), 268-272; англ. пер.: V. F. Lazutkin, "The width of the instability zone around separatrices of a standard mapping", Soviet Math. Dokl., 42:1 (1991), 5-9.

[11] Я.Г. Синай, "Несколько точных результатов об убывании корреляций”, Дополнение в кн. Г. М. Заславский, Статистическал необратимость в нелинейных системах, Соврем. проблемы физики, Наука, М., 1970, 124-139.

[12] В.В. Козлов, "Расщепление сепаратрис и рождение изолированных периодических решений в гамильтоновых системах с полутора степенями свободы", УМH, 41:5 (1986), 177-178; англ. пер.: V. V. Kozlov, "The splitting of separatrices and the generation of isolated periodic solutions in Hamiltonian systems with one-and-a-half degrees of freedom", Russian Math. Surveys, 41:5 (1986), 149-150. 
[13] D. V. Treschev, "Hyperbolic tori and asymptotic surfaces in Hamiltonian systems", Russian J. Math. Phys., 2:1 (1994), 93-110.

[14] С. А. Довбыш, "Расщепление сепаратрис и рождение изолированных периодических решений в гамильтоновых системах с полутора степенями свободы", УМН, 44:2 (1989), 229-230; англ. пер.: S. A. Dovbysh, "The splitting of separatrices and the generation of isolated periodic solutions in Hamiltonian systems with one and a half degrees of freedom", Russian Math. Surveys, 44:2 (1989), 281-282.

[15] C. Simó, D. Treschev, "Stability islands in the vicinity of separatrices of nearintegrable symplectic maps", J. Discrete Contin. Dyn. Syst. (to appear).

[16] В.И. Арнольд, "О неустойчивости динамических систем со многими степенями свободы", Докл. АН СССР, 156:1 (1964), 9-12; англ. пер.: V. I. Arnol'd, "Instability of dynamical systems with several degrees of freedom", Soviet Math. Dokl., 5 (1964), $581-585$.

[17] Н.Н. Нехорошев, "Экспоненциальная оценка времени устойчивости гамильтоновых систем близких к интегрируемым", УМН, 32:6(198) (1977), 5-66; англ. пер.: N. N. Nekhoroshev, "An exponential estimate of the time of stability of nearlyintegrable hamiltonian systems", Russian Math. Surveys, 32:6 (1977), 1-65.

[18] R. Douady, "Stabilité ou instabilité des points fixes elliptiques", Ann. Sci. École Norm. Sup. (4), 21:1 (1988), 1-46.

[19] J.-P. Marco, D. Sauzin, "Stability and instability for Gevrey quasi-convex nearintegrable Hamiltonian systems", Publ. Math. Inst. Hautes Études Sci., 96:1 (2003), 199-275.

[20] E. Fontich, P. Martin, "Arnold diffusion in perturbations of analytic integrable Hamiltonian systems", Discrete Contin. Dynam. Systems, 7:1 (2001), 61-84.

[21] G. Gallavotti, G. Gentile, V. Mastropietro, "Hamilton-Jacobi equation, heteroclinic chains and Arnol'd diffusion in three time scale systems", Nonlinearity, 13:2 (2000), 323-340.

[22] U. Bessi, "An approach to Arnold's diffusion through the calculus of variations", Nonlinear Anal., 26:6 (1996), 1115-1135.

[23] C. Simó , C. Valls, "A formal approximation of the splitting of separatrices in the classical Arnold's example of diffusion with two equal parameters", Nonlinearity, 14:6 (2001), 1707-1760.

[24] J. N. Mather, "Arnold duffision. I: Announcement of results", J. Math. Sci. (N. Y.), 124:5 (2004), 5275-5289; пер. с русск.: Дж. Н. Мэзер, “Диффузия Арнольда, I: анонс результатов", Современная математика. Фундаментальные направления, 2, МАИ, М., 2003, 116-130.

[25] G. N. Piftankin, "Diffusion speed in the Mather problem", Nonlinearity, 19:11 (2006), 2617-2644.

[26] S. Bolotin, D. Treschev, "Unbounded growth of energy in nonautonomous Hamiltonian systems", Nonlinearity, 12:2 (1999), 365-388.

[27] A. Delshams, R. de la Llave, T. M. Seara, "A geometric approach to the existence of orbits with unbounded energy in generic periodic perturbations by potential of generic geodesic flows on $T^{2}$, Comm. Math. Phys., 209:2 (2000), 353-392.

[28] V. Kaloshin, "Geometric proofs of Mather's connecting and accelerating theorems", Topics in dynamics and ergodic theory (Katsiveli, Ukraine, 2000), London Math. Soc. Lecture Note Ser., 310, Cambridge Univ. Press, Cambridge, 2003, 81-106.

[29] A. Delshams, R. de la Llave, T. M. Seara, "Orbits of unbounded energy in quasiperiodic perturbations of geodesic flows", Adv. Math., 202:1 (2006), 64-188.

[30] L. Chierchia, G. Gallavotti, "Drift and diffusion in phase space", Ann. Inst. H. Poincaré. Phys. Théor., 60:1 (1994), 1-144.

[31] D. Treschev, "Multidimensional symplectic separatrix maps", J. Nonlinear Sci., 12:1 (2002), 27-58. 
[32] D. Treschev, "Evolution of slow variables in a priori unstable Hamiltonian systems", Nonlinearity, 17:5 (2004), 1803-1841.

[33] M. Berti, P. Bolle, "Diffusion time and splitting of separatrices for nearly integrable isochronous Hamiltonian systems", Atti Accad. Naz. Lincei Cl. Sci. Fis. Mat. Natur. Rend. Lincei (9) Mat. Appl., 11:4 (2000), 235-243.

[34] J. Cresson, C. Guillet, "Periodic orbits and Arnold diffusion", Discrete Contin. Dyn. Syst., 9:2 (2003), 451-470.

[35] M. Berti, L. Biasco, P. Bolle, "Drift in phase space: A new variational mechanism with optimal diffusion time", J. Math. Pures Appl. (9), 82:6 (2003), 613-664.

[36] B. Berti, L. Biasco, P. Bolle, "Optimal stability and instability results for a class of nearly integrable Hamiltonian systems", Atti Accad. Naz. Lincei Cl. Sci. Fis. Mat. Natur. Rend. Lincei (9) Mat. Appl., 13:2 (2002), 77-84.

[37] Z. Xia, "Arnold diffusion: a variational construction", Doc. Math. J. DMV Extra Volume ICM II, 1998, 867-877.

[38] C.-Q. Cheng, J. Yan, "Existence of diffusion orbits in a priori unstable Hamiltonian systems", J. Differential Geom., 67:3 (2004), 457-517.

[39] A. Delshams, R. de la Llave, T. M. Seara, A geometric mechanism for diffusion in Hamiltonian systems, http://www.ma.utexas.edu/mp_arc/e/03-182.ps.

[40] A. Delshams, R. de la Llave, T. M. Seara, A geometric mechanism for diffusion in Hamiltonian systems overcoming the large gap problem: heuristic and rigorous verification on a model, Mem. Amer. Math. Soc., 179:844, Amer. Math. Soc., Providence, RI, 2006.

[41] Дж. Д. Биркгоф, Динамические системы, Гостехиздат, М.-Л., 1941; пер. с англ.: G. D. Birkhoff, Dynamical systems, Amer. Math. Soc., New York, 1927.

[42] J. Moser, "The analytic invariants of an area-preserving mapping near a hyperbolic fixed point", Comm. Pure Appl. Math., 9:4 (1956), 673-692.

[43] A. Banyaga, R. de la Llave, C.E. Wayne, "Cohomology equations near hyperbolic points and geometric versions of Sternberg linearization theorem", J. Geom. Anal., 6:4 (1996), 613-649.

[44] Д. В. Трещев, Введение в теорию возмущений гамильтоновых систем, Фазис, M., 1998.

[45] С. Л. Зиглин, "Ветвление решений и несуществование первых интегралов в гамильтоновой механике. I, II", Функи. анализ и его прилож., 16:3 (1982), 30-41; 17:1 (1983), 8-23; англ. пер.: S. L. Ziglin, "Branching of solutions and nonexistence of first integrals in Hamiltonian mechanics. I, II", Funct. Anal. Appl., 16:3 (1982), 181-189; 17:1 (1983), 6-17.

[46] A. Pronin, D. Treschev, "On the inclusion of analytic maps into analytic flows", Regul. Chaotic Dyn., 2:2 (1997), 14-24.

[47] D. Treschev, "Width of stochastic layers in near-integrable two-dimensional symplectic maps", Phys. D, 116:1-2 (1998), 21-43.

[48] В. И. Арнольд, В. В. Козлов, А. И. Нейштадт, "Математические аспекты классической и небесной механики", Итоги науки и техники. Соврем. проблемы матем. Фундам. напр., 3, ВИНИТИ, М., 1985, 5-304; англ. пер.: V. I. Arnol'd, V.V. Kozlov, A.I. Neǐshtadt, Mathematical aspects of classical and celestial mechanics, Dynamical systems, III, 3rd ed., Encyclopaedia Math. Sci., 3, Springer, Berlin, 2006.

[49] S. M. Graff, "On the conservation of hyperbolic tori for Hamiltonian systems", J. Differential Equations, 15:1 (1974), 1-69.

[50] E. Zehnder, "Generalized implicit function theorem with applications to some small divisor problems. I, II", Comm. Pure Appl. Math., 28:1 (1975), 91-140; 29:1 (1976), 49-111. 
[51] H. Poincaré, Les métodes nouvelles de la mécanique céleste, 1-3, Gauthier-Villars, Paris, 1892; 1893; 1899.

[52] A. Delshams, P. Gutiérrez, "Splitting potential and Poincaré-Melnikov method for whiskered tori in Hamiltonian systems", J. Nonlinear Sci., 10:4 (2000), 433-476.

[53] V.G. Gelfreich, "Separatrices splitting for the rapidly forced pendulum", Seminar on Dynamical Systems (St. Petersburg, Russia, 1991), Progr. Nonlinear Differential Equations Appl., 12, Birkhäuser, Basel, 1994, 47-67.

[54] В.Ф. Лазуткин, Расщепление сепаратрис в стандартном отображении Чирикова, деп. в ВИНИТИ № 6372-84.

[55] V. G. Gelfreich, V.F. Lazutkin, N. V. Svanidze, "A refined formula for the separatrix splitting for the standard map", Phys. D, 71:1-2 (1994), 82-101.

[56] В. Гельфрейх, В. Лазуткин, "Расщепление сепаратрис: теория возмущений, экспоненциальна малость", УМH, 56:3 (2001), 79-142; англ. пер.: V. G. Gelfreich, V.F. Lazutkin, "Splitting of separatrices: perturbation theory and exponential smallness", Russian Math. Surveys, 56:3 (2001), 499-558.

[57] A. Delshams, T. M. Seara, "An asymptotic expressions for the splitting of separatrices of the rapidly forced pendulum", Comm. Math. Phys., 150:3 (1992), 433-463.

[58] D. Treschev, "An averaging method for Hamiltonian systems, exponentially close to integrable ones", Chaos, 6:1 (1996), 6-14.

[59] D. Treschev, "Separatrix splitting for a pendulum with rapidly oscillating suspension point", Russian J. Math. Phys., 5:1 (1997), 63-98.

[60] S. Aubry, "The twist map, the extended Frenkel-Kontorova model and the devil's staircase", Phys. D, 7:1-3 (1983), 240-258.

[61] R.S. MacKay, "A renormalization approach to invariant circles in area-preserving maps", Phys. D, 7:1-3 (1983), 283-300.

[62] J. N. Mather, "Non-existence of invariant circles", Ergodic Theory Dynam. Systems, 4:2 (1984), 301-311.

[63] V.G. Gelfreich, "Reference systems for splitting of separatrices", Nonlinearity, 10:1 (1997), 175-193.

[64] G.D. Birkhoff, "Surface transformations and their dynamical applications", Acta Math., 43 (1920), 1-119.

[65] M. R. Herman, Sur les courbes invariantes par les difféomorphismes de l'anneau, vol. I, Astérisque, 103-104, Soc. Math. France, Paris, 1983.

[66] D. Treschev, "Trajectories in a neighborhood of asymptotic surface of a priori unstable Hamiltonian systems", Nonlinearity, 15:6 (2002), 2033-2052.

Г. Н. Пифтанкин (G. N. Piftankin)

Поступила в редакцию

Московский государственный университет

01.02.2007

им. М.В. Ломоносова

\section{Д. В. Трещёв (D. V. Treschev)}

Математический институт им. В. А. Стеклова РАН

E-mail: treschev@mi.ras.ru 